

\section{EFFICIENT HEALTHCARE LOGISTICS WITH A HUMAN TOUCH}

Maartje van de Vrugt 


\section{Dissertation committee}

Chairman \& secretary: $\quad$ Prof. dr. P.M.G. Apers

University of Twente, Enschede, the Netherlands

Promotor: Prof. dr. R.J. Boucherie

University of Twente, Enschede, the Netherlands

Members: Dr. M. Bessems

Jeroen Bosch Ziekenhuis, 's Hertogenbosch, the Netherlands

Prof. dr. E. Demeulemeester

KU Leuven, Leuven, Belgium

Prof. dr. ir. E.W. Hans

University of Twente, Enschede, the Netherlands

Prof. dr. J.L. Hurink

University of Twente, Enschede, the Netherlands

Prof. dr. E.K. Lee

Geogia Institute of Technology, Atlanta, United States

Prof. dr. R. van der Mei

VU University, Amsterdam, the Netherlands

Dr. I. Ziedins

University of Auckland, Auckland, New Zealand

Ph.D. thesis, University of Twente, Enschede, the Netherlands

Center for Telematics and Information Technology (No. 16-389, ISSN 1381-3617).

Center for Healthcare Operations Improvement and Research.

Beta Research School for Operations Management and Logistics (No. D197).

The distribution of this thesis is financially supported by the Jeroen Bosch Ziekenhuis,

's Hertogenbosch, the Netherlands.

Printed by Ipskamp printing, Enschede, the Netherlands.

Cover design: Judith van de Vrugt.

Copyright (c) 2016, Maartje van de Vrugt, Enschede, the Netherlands.

All rights reserved. No part of this publication may be reproduced without the prior written permission of the author.

ISBN 978-90-365-4115-2

DOI $10.3990 / 1.9789036541152$ 


\title{
EFFICIENT HEALTHCARE LOGISTICS WITH A HUMAN TOUCH
}

\author{
PROEFSCHRIFT
}

\author{
ter verkrijging van \\ de graad van doctor aan de Universiteit Twente, \\ op gezag van de rector magnificus, \\ Prof. dr. H. Brinksma, \\ volgens besluit van het College voor Promoties \\ in het openbaar te verdedigen \\ op vrijdag 1 juli 2016 om 14.45 uur
}

door

Noëlle Maria van de Vrugt

geboren op 18 december 1988

te Amersfoort, Nederland 
Dit proefschrift is goedgekeurd door de promotor:

Prof. dr. R.J. Boucherie 


\section{Voorwoord}

Toen ik bijna tien jaar geleden aan mijn Bachelor Toegepaste Wiskunde begon in Enschede, heb ik (volgens mij als enige van ons jaar) heel hard geroepen dat ik écht met vijf jaar weer uit Enschede weg zou zijn. Omdat ik twijfelde of de studie niet te moeilijk voor mij zou zijn, ben ik heel bewust het eerste half jaar niet bij commissies of sportverenigingen gegaan. Dat ik ooit een proefschrift zou schrijven over dit vakgebied had ik nooit verwacht. Ik ben erg dankbaar voor de kansen die mij geboden zijn de afgelopen jaren, en voor de zeer gewaardeerde samenwerkingen, support en broodnodige afleiding die ik gehad heb in de aanloop naar en tijdens mijn promotietraject. Zonder mijn dankbaarheid voor de steun van alle anderen tekort te doen, wil ik een aantal mensen hiervoor in het bijzonder bedanken.

Richard, dit promotietraject was er nooit gekomen zonder jouw enthousiaste begeleiding bij het schrijven van mijn onderzoeksvoorstel, inmiddels al wat jaren geleden. Ik heb onze samenwerking enorm gewaardeerd en jouw passie voor de wiskunde en haar toepassingen is inspirerend. Mijn promotietraject had een wat onzekere start, en daarin heb ik ontzettend veel aan jouw begeleiding, enthousiasme en goede ideeën gehad. Hoewel je het misschien niet eens door hebt gehad, heb je me ook enorm vooruit geholpen met tennis; ook dank daarvoor.

Additionally, I would like to thank my committee members for their time invested in my $\mathrm{PhD}$ thesis and defense, and their valuable comments. Erik Demeulemeester, Erwin Hans, Eva Lee, Ilze Ziedins, Johann Hurink, Maud Bessems, and Rob van der Mei: your efforts are very much appreciated.

Bij het onderzoek in dit proefschrift is een groot aantal co-auteurs en behulpzame collega's betrokken geweest, die ik allen hartelijk wil bedanken. Richard, van jouw scherpe schrijfstijl heb ik veel geleerd, dank voor de bijdragen voor alle hoofdstukken in mijn proefschrift. For Chapter 2, I thank Thomas, Maartje and David for their contributions. Hoofdstuk 3 was nooit tot stand gekomen zonder de input van Saskia, Eric, Thom, Yvonne en alle betrokkenen uit het C-gebouw van het Jeroen Bosch Ziekenhuis (JBZ). Aleida, ik ben ontzettend blij dat ons literatuuronderzoek (Hoofdstuk 4) er uiteindelijk gekomen is, dank daarvoor. Nelly, dank voor het tot een artikel brengen van mijn afstudeerwerk en de fijne samenwerking daarvoor (Hoofdstuk 5). Tineke, Mathijn, Maud, Margo, Marjolein, John (Peeters); het succes van onderzoek in Hoofdstuk 6 is grotendeels door jullie bewerkstelligd. Petra, Peter, Tiny en John (de Laat): dank voor jullie bijdragen aan het onderzoek in Hoofdstuk 7. For Chapter 8 I would like to acknowledge the hard work of Sam, Wietske, Stef, Wouter, Mark, 
Dirk Jan, Erwin and Johann. Jan en collega's van de poli Chirurgie, dank voor jullie bijdrage aan het onderzoek in Hoofdstuk 9, en Corine dank voor jouw werk tijdens en na je afstudeerproject. Ilze, thank you very much for your contribution to the research in Chapter 11, and the very much appreciated time we spend together, both inside and outside the office. Hiernaast ben ik ook Annemarie, Walter, Adriaan en Niek (Gertsen) heel dankbaar voor het aanleveren van de datasets die mijn onderzoek vele malen leuker en makkelijker gemaakt hebben.

Voor de kansen en mogelijkheden die het JBZ mij geboden heeft, ben ik heel dankbaar. Uit het grote aantal JBZ-ers dat hierboven genoemd is, blijkt al dat ik veel steun gehad heb aan mijn collega's daar. Ook mijn collega's bij Kwaliteit en Veiligheid, de collega's bij afdeling B2-management, en onder andere Marjoke en Jitske van Capaciteitsmanagement: dank voor de altijd gezellige tijden en de geboden helpende handen. In het bijzonder wil ik Saskia en Eric bedanken; mijn promotietraject was een stuk minder aangenaam geweest zonder jullie begeleiding in het JBZ, bedankt! Het is grotendeels jullie verdienste geweest dat ik me vanaf dag één thuis gevoeld heb in het ziekenhuis en dat ik nooit verlegen heb gezeten om leuke projecten tijdens en naast mijn promotietraject. Ook de Raad van Bestuur van het JBZ wil ik bedanken voor het in mij gestelde vertrouwen.

Op de Universiteit Twente wil ik mijn SOR, DWMP en CHOIR collega's bedanken voor de altijd gezellige tijden. Zonder mijn waardering voor de andere collega's tekort te doen, noem ik toch een paar collega's in het bijzonder.

Aleida, ik heb ontzettend genoten van onze samenwerking en wandelingen tijdens congressen en ben blij dat we nog steeds met elkaar omgaan ondanks alles wat je door mij hebt moeten doorstaan. Door jouw perfectionisme is ons paper vele malen mooier geworden, en jouw kwaliteit om snel te verwoorden wat we beiden dachten, heeft ons paper vaak enorm geholpen.

Niek, hoewel ik in eerste instantie niet echt blij was dat ik bij jou op de kamer kwam, heb ik mij geweldig vermaakt met jou als kamergenoot, zeker tijdens onze vele muzikale sessies en het congres in Parijs.

Pim, bedankt voor al je hulp bij mijn Matlab-ellende, ook al was het soms voor jou diep in de nacht.

Nelly, dank voor alle goede adviezen en je gezelligheid in de afgelopen jaren.

Daarnaast wil ik nog al mijn kamergenoten en CHOIR collega's bedanken voor de geboden hulp en gezelligheid, zowel onder als buiten werktijden. Maartje, Nikky, Theresia en Egbert, dank voor jullie inspirerende proefschriften (die ik als voorbeelden gebruikt heb de laatste maanden) en de goede tijd, ook tijdens congressen en na jullie vertrek bij CHOIR. Nardo en Gréanne, hoewel we het nooit voor elkaar gekregen hebben, toch bedankt voor de hulp met mijn simulaties. Nardo, Ingeborg, Sem en Gréanne, bedankt voor de input voor onder andere de layout van mijn proefschrift. 
During my PhD-project I got the wonderful opportunity to travel to New Zealand twice, to work together with Ilze Ziedins at the University of Auckland. Ilze, thank you so much for making me feel at home so far from home. I have greatly enjoyed working with you and sincerely hope that we keep in touch after our paper is published. Additionally, I thank my colleagues and office mates there, among who Niffe, Mark, David, Joei, and Chris, for the wonderful time I had at the university of Auckland. Mijn eerste reis naar Nieuw Zeeland was niet mogelijk geweest zonder de beurs van het Jo Kolk Studiefonds van de Vereniging van vrouwen met hogere opleiding (VVAO), waarvoor ik enorm dankbaar ben. Ook de interesse die getoond is vanuit de afdeling Enschede van de VVAO is hartverwarmend.

Last but not least wil ik natuurlijk mijn familie en vrienden bedanken voor alle steun en broodnodige afleiding die jullie me geboden hebben de afgelopen jaren. Zonder de gezelligheid van Huize Steunzool, Damesdispuut Dionysus, T.C. Ludica, de wiskundemeisjes en de Limoncello-club (ik weiger onze nieuwste appgroepnaam hier te gebruiken) waren de afgelopen jaren enorm saai geweest, dus ontzettend bedankt! Anouk en Lotte, 'we' worden in rap tempo steeds burgerlijker, maar ik kijk enorm uit naar de tijden op onze boot op een Mediterraanse zee en alle andere momenten om samen met jullie door te brengen. Mirel, dank je wel voor alle gezellige avondjes en goede gesprekken. Ik ben bijzonder trots en dankbaar dat je mijn paranimf wil zijn, zelfs als dat glitters met zich meebrengt. Paps, mams en Pim, Judith (sorry Pu) en Vincent: dank voor het bieden van luisterende oren, gezelligheid, slaapplaatsen en steun waar nodig. Lieve grote kleine zus, super bedankt dat je mijn paranimf (elfje) wil zijn en dat je de geweldige omslag van dit proefschrift ontworpen hebt.

Maartje

Enschede, juni 2016 

1 Research motivation and outline 1

1.1 Developments in healthcare . . . . . . . . . . . . . 1

1.2 Efficient healthcare logistics with a human touch . . . . . . . 2

1.3 Operations Research in healthcare . . . . . . . . . . . . . . . . 4

1.4 Jeroen Bosch hospital . . . . . . . . . . . . . . . . . . . . . . 4

1.5 Thesis outline .................. 5

I Operations Research applied to hospital wards $\quad 9$

2 OR for hospital wards and its integration into practice 11

2.1 Introduction . . . . . . . . . . . . . . . . . . 11

2.2 Hospital ward types and terminology . . . . . . . . . . . . . 11

2.3 OR model types . . . . . . . . . . . . . . . . . . . . . . . . . . . . . . . . . . . 20

2.4 Ward-related OR models . . . . . . . . . . . . . . 25

2.5 Illustrations of OR model use . . . . . . . . . . . . . . . . . . . . . . . . . . . . . . 34

2.6 Implemented OR results . . . . . . . . . . . . . . . . . . . . . . . 40

2.7 Challenges and directions further research . . . . . . . . . . . 42

3 Balancing occupancy over medical wards $\quad \mathbf{4 5}$

3.1 Introduction . . . . . . . . . . . . . . . . . . . . . 45

3.2 Before intervention . . . . . . . . . . . . . . . . . 46

3.3 Intervention . . . . . . . . . . . . . . . . . . . . . . . . . 47

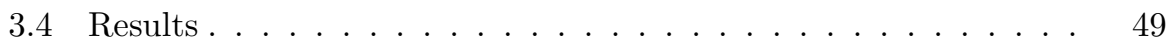

3.5 Additional improvements . . . . . . . . . . . . . . . . 50

3.6 Conclusion ....................... 50

II Online appointment scheduling 53

4 The state of the art in online appointment scheduling 55

4.1 Introduction . . . . . . . . . . . . . . . . . . 55

4.2 Scope and taxonomy . . . . . . . . . . . . . . . 56

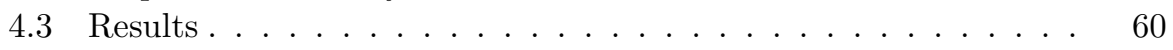

4.4 Appointment scheduling models . . . . . . . . . . . . . . . . 78

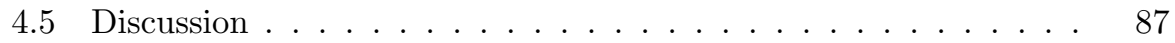

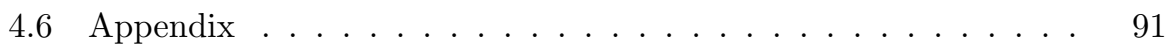


5 Blocking probabilities in queues with advance reservation $\quad \mathbf{9 3}$

5.1 Introduction . . . . . . . . . . . . . . . . . . . . . . . . . 93

5.2 The model and special cases . . . . . . . . . . . . . . . . . . . . . 94

5.3 The effect of advance reservation . . . . . . . . . . . . . 100

5.4 Numerical results . . . . . . . . . . . . . . . . . . . . . . . . . . . . . . . . . . . . . . . 101

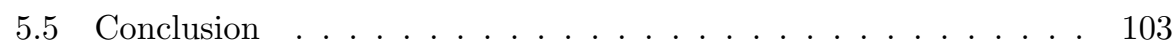

III Patient appointment schedules 105

6 Rapid diagnoses at the breast center $\quad 107$

6.1 Introduction . . . . . . . . . . . . . . . . . 107

6.2 Related literature . . . . . . . . . . . . . . . . . . 109

6.3 Access time . . . . . . . . . . . . . . . . . . . . . . . . . . . . . . . . . . 110

6.4 Time to diagnosis . . . . . . . . . . . . . . . . . . . . . . . . . . . . . . . . . . . . . 114

6.5 Waiting time . . . . . . . . . . . . . . . . 116

6.6 Conclusion . . . . . . . . . . . . . . . . . . 119

6.7 Appendix: Discrete time queueing model . . . . . . . . . . . . . . 120

6.8 Appendix: Discrete event simulation model . . . . . . . . . . 121

7 Appointments versus walk-in at the plaster room 125

7.1 Introduction . . . . . . . . . . . . . . . . . . . . . 125

7.2 Simulation model . . . . . . . . . . . . . . . . . . . . . . . . . . . . . . . . . 126

7.3 Results. . . . . . . . . . . . . . . . . . . . . . . . . . . . . . . . . . . . 129

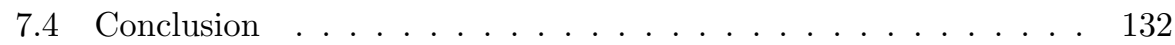

IV Optimizing doctor schedules 135

8 Scheduling gynecologists to balance patients' access times $\quad \mathbf{1 3 7}$

8.1 Introduction . . . . . . . . . . . . . . . . . . . . . . . . . . . . . . . 137

8.2 Model . . . . . . . . . . . . . . . . . . . 139

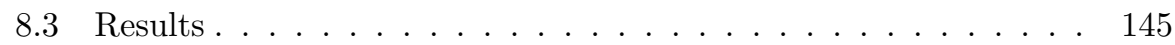

8.4 Conclusion . . . . . . . . . . . . . . . . 150

8.5 Appendix: Pseudo-code of the heuristics . . . . . . . . . . . . . . 152

8.6 Appendix: Parameters for the case study . . . . . . . . . . 153

9 Static and dynamic surgeon scheduling $\quad \mathbf{1 5 7}$

9.1 Introduction . . . . . . . . . . . . . . . . . . . . . . . . . . . 157

9.2 Mathematical model . . . . . . . . . . . . . . . . . . . . 158

9.3 Application ........................... 164

9.4 Discussion . . . . . . . . . . . . . . . . . . . . . . . . . . . . . . . . . . . . . 167

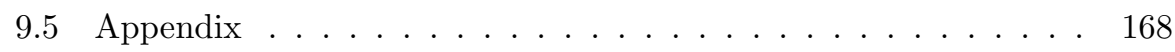


V Optimizing throughput at the ED

10 Assigning treatment rooms at the emergency department $\quad 173$

10.1 Introduction . . . . . . . . . . . . . . . . . . . . . . . . . . . . . 173

10.2 Related literature . . . . . . . . . . . . . . . . . . . . . . 174

10.3 The Model . . . . . . . . . . . . . . . . . . . . . 175

10.4 Analytical results . . . . . . . . . . . . . . . . . . . . . . 177

10.5 Numerical results . . . . . . . . . . . . . . . . . . . . . . . . . . . . . . . . . . . 178

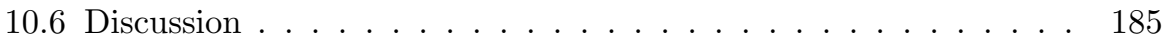

10.7 Appendix A The generator . . . . . . . . . . . . . . . 186

10.8 Appendix B Conditional performance measures . . . . . . . . . . 187

11 Sequentially assigning and prioritizing patients at EDs 191

11.1 Introduction . . . . . . . . . . . . . . . . . . . . . . . . . . . . . . . . . 191

11.2 Related literature . . . . . . . . . . . . . . . . . . . . . 192

11.3 Extreme case analyses . . . . . . . . . . . . . . . . . . . 193

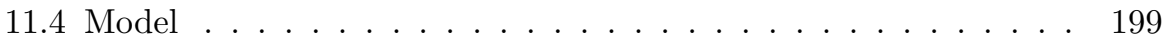

11.5 Results . . . . . . . . . . . . . . . . . . . . . . . . . . . . . 202

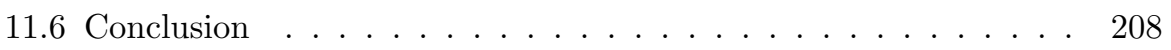

12 Conclusion and outlook $\quad 209$

$\begin{array}{ll}\text { Bibliography } & 213\end{array}$

$\begin{array}{ll}\text { Acronyms } & \mathbf{2 5 5}\end{array}$

$\begin{array}{ll}\text { Summary } & 257\end{array}$

$\begin{array}{ll}\text { Samenvatting } & \mathbf{2 6 1}\end{array}$

$\begin{array}{lr}\text { About the author } & 266\end{array}$

$\begin{array}{ll}\text { List of publications } & 268\end{array}$ 



\section{Research motivation and outline}

\subsection{Developments in healthcare}

Good health significantly contributes to happiness and well-being, but is also of important economic value as healthy people tend to live longer and are more productive [520]. In the last centuries, healthcare and healthcare technology have gone through several remarkable developments, such as: the first study of human anatomy (16th century), the discovery of vaccines and anti-bacterial sprays (19th century), and the discovery of X-ray imaging (1895) [474]. More recent developments are for example surgery robots, several new imaging techniques, and nanomedicine. From the year 1850, these developments have contributed to an increase in life expectancy, which has doubled since the 19th century [422].

The rapidly rising healthcare expenditures have been internationally recognized about 50 years ago. Anderson et al. [14] for example state in 2005: 'a cycle of unsustainable spending growth followed by fervent cost containment initiatives has been a regular feature of the health care landscape for the past half-century.' The growth of the expenditures cannot be explained by the aging population alone; medical and technological advances in healthcare contribute to increasing healthcare expenditures and increasing numbers of hospitalized patients [102]. Better medical equipment can support patients' primary life functions longer. Better medicine can cure more illnesses that would have been lethal otherwise. Better diagnostic technologies can detect more defects at an earlier stage, which may result in 'over diagnostics and over treatment'; patients are treated for a defect that would not have resulted in any complaints. Together with the aging population, this increased demand for healthcare puts the healthcare sector under pressure regarding both costs and resources; $12 \%$ of the Dutch labor force worked in healthcare in 2012, which will increase to $25 \%$ in 2030 if the increase in the demand for care continues its current trend [370]. However, medical and technological advances also alleviate the pressure on the healthcare sector, for example by significantly reducing the number of hospitalization days per capita [236], or possibly by introducing robots that take over certain nursing tasks.

Concurrent to the mentioned technological developments, patients are becoming increasingly educated and informed regarding their health, possibilities for treatments, and where to go for these treatments. In the Netherlands, each year a 
'hospital top 100' is published in a national newspaper, in which all non-academic and non-specialized hospitals are compared according to certain performance indicators [509]. There are several other national and international organizations assessing for example the quality of the provided care, accessibility of outpatient clinics, and throughput times at the emergency department. The Dutch 'Health Care Inspectorate' for example requested that each hospital provided information on 307 medical and logistical performance indicators in 2015 [475]. Treating more informed patients has changed the care process into a 'patient-centered' process; patients determine their treatment together with the doctor, resulting in more individual, customized care. Hospitals anticipate on this trend by organizing care more according to diagnoses instead of medical specialties, but this requires much cooperation between the specialties, for example in aligning doctor schedules.

Doctor schedules determine to a large extent the performance of a hospital with respect to for example accessibility. Therefore, it is striking that in most (Dutch) hospitals it is still common practice that one of the doctors creates the schedules. Not only does this practice reduce a doctor's time spend on treating patients, but academics also frequently proved the difficulty of determining good schedules, see for example [293]. Medical doctors have a medical education, took an oath to provide the best possible care to all their patients, and might therefore make suboptimal decisions in solving logistical problems.

In summary, medical and technological advances can both support and hinder the healthcare sector. Healthcare is becoming more and more individual, patient-centered, which forces different medical departments to align their activities. Despite large technological advances there is still much potential to improve logistics and logistical support in hospitals.

\subsection{Efficient healthcare logistics with a human touch}

The broad term 'logistics' means according to the Cambridge Online Dictionary 'the careful organization of a complicated activity so that it happens in a successful and effective way'. Besides organizing a complicated activity, other definitions additionally mention 'implementation of a complex operation'. Logistical decision making is prevalent at different time horizons, ranging from long-term strategic to immediate online decision making. Each different time horizon implies different types of possibilities, decisions, and solution approaches.

The term 'healthcare logistics' encompasses many different focus areas, such as ambulance dispatching, material planning, and appointment scheduling. Healthcare logistics often involve cooperation and communication between multiple practitioners from different departments, or even different healthcare facilities. Different departments of one hospital often make logistical decisions in a decentralized fashion, but their patient inflows are strongly inter-related. For example, patients may access the hospital at the emergency department, require diagnostic tests, possibly require surgery, and are admitted to an inpatient 
ward. In this thesis we focus on logistical challenges on different time horizons at different departments of a hospital: inpatient admission planning; patient appointment scheduling and resource scheduling for outpatient departments; and length of stay reduction at an emergency department. In all of these challenges, different practitioners and/or departments cooperate to provide the best possible care. Furthermore, in this thesis also the implementation part of the definition of logistics is important; we present inventive, practically relevant applied mathematical approaches that are suitable for implementation in the complex hospital operations.

Part of the definition of logistics refers to success and effectiveness of the way the logistics are organized. In a healthcare setting, this implies that the right treatment should be provided to the right patient at the right time. For hospitals with superfluous capacity, this would imply that all necessary resources, such as medical equipment and care practitioners, are available at the moment patients request them. However, the capacity of the healthcare system is limited as buffering capacity against the varying patient demand is not possible and too expensive. Therefore, in practice it is for example difficult to schedule all appointments for one patient with different practitioners on a single day, or schedule appointments within the medically preferred time window. Possible consequences are a reduction of the quality of care, and less smooth treatment processes for patients.

Logistical efficiency lacks a uniform definition, and depends on the performance measures and objectives for each logistical process. In this thesis we optimize logistical efficiency in three different ways: (1) we balance utilization, to prevent that patients have to be deferred at wards; (2) we align or optimize resource schedules, to minimize patients' waiting and access times; and (3) we optimize room assignments and working routines to reduce patients' length of stay at the emergency department. Optimizing the efficiency of healthcare logistics often improves both patient-friendliness and quality of care through better accessibility and alignment of the appointments, and may provide the hospital with the possibility to treat more patients with the same capacity.

Although from a mathematical viewpoint healthcare logistics are similar to logistics in production factories or service industries, a hospital is not a factory that produces products, and doctors and nurses are not assembly robots; curing patients still requires much interaction between patients and practitioners. Therefore, practitioners who are structurally overloaded, or otherwise unhappy or unfocused in performing their jobs, may be a risk to the quality of care. To this end, besides patient-centered logistical efficiency, in this thesis we incorporate medical doctor and nursing staff objectives and preferences into our optimization models. We take overtime into account, balance the workload, or maximize the compliance with doctors' preferences. Additionally, by organizing processes efficiently and/or computerizing scheduling tasks, doctors are alleviated from ancillary tasks so they can spend more time on actual patient care. Therefore, the logistical approaches presented in this thesis all have a human touch, and create 
both patient- and doctor-centered solutions.

\subsection{Operations Research in healthcare}

The term 'Operations Research' (OR) entails a mathematical discipline that aids decision making in mostly logistical applications. The field of OR entails many different methodologies, such as queueing theory and discrete event simulation. An overview of the commonly used methods, including introductory examples, is provided in Section 2.3.1. OR methodologies are applied to many different application areas, such as production or service industries. Chapter 4 provides several examples of applied OR methods for appointment scheduling specifically.

The first application of OR methodologies to healthcare processes dates back to 1952, when Bailey [26] investigated several outpatient appointment scheduling rules. Since then, OR researchers have increasingly studied healthcare applications, such as creating schedules for the operating theater, nurses, and outpatients' appointments. Besides the applications in this thesis, the recent review paper by Hulshof et al. [242] provides many examples of healthcare applications studied using OR.

OR methodologies may support healthcare professionals in making better decisions concerning planning and capacity issues and improving efficiency in the delivery of healthcare. The advantage of OR models for healthcare is that possible interventions can be evaluated in a safe virtual environment, reducing the risk of implementing an intervention that appears to be counter-productive, as we elaborate upon in Section 2.3.2.

Despite the vast amount of academic papers on this topic and the large potential of OR methodologies, it appears that the actual implementation of OR models and/or results in healthcare practice is rare. This thesis aims to bridge the gap between theory and practice, as it consists both of theoretical research and implementation-oriented case studies performed at our partnering hospital. Chapters 3 and 6 of this thesis contain research implemented at our partnering hospital, and show the results of these interventions for practice. The hospital is considering implementing results of Chapters 7, 8 and 9. In Section 2.6 we elaborate on the experienced problems with implementing research results, and the lessons learned from the research conducted for this thesis and the research included Chapter 2. Although the research in this thesis is tailored to the case of our partnering hospital, the methods are generic and readily adapted to other hospital settings, and are in some cases even applicable outside the healthcare domain.

\subsection{Jeroen Bosch hospital}

The research presented in this thesis is to a large extent inspired by problems raised by the staff of the Jeroen Bosch hospital (JBH). The JBH is a 'top-clinical' hospital, a relatively large non-university teaching hospital, and is part of the as- 
sociation of tertiary medical teaching hospitals. The first basis for the JBH dates back to 1274, with the opening of the Groot Gasthuis in the center of the city 's Hertogenbosch [249]. The JBH as it is known today opened its doors in 2011, as a merge of three large hospitals in the province of Noord-Brabant (southern region in the Netherlands). In 2014, the JBH had 760 registered beds, accommodated 57,503 patients, and provided 520,940 outpatient clinic consultations [250]. The JBH employed 3,617 staff members (2,688 fte), and hired 257 (223 fte) medical specialists, which made the JBH the largest employer in its region. Almost all medical specialties are represented at the hospital.

The JBH aims to be the most patient-centered and safe hospital of the Netherlands. To this end, the hospital focuses on the following four core values: safety, hospitality, openness, and innovation. To ensure the quality of care, the JBH aims that its offered care complies with the six dimensions of quality: safe, effective, efficient, patient-centered, timely, and equitable. The JBH aims to deliver care timely and efficiently, and every patient should receive the same quality of care. The medical specialists and nurses let patients do and decide as much as possible themselves, what the JBH calls 'patient empowerment'. All provided care is based on best practices and scientific research.

The JBH is a teaching hospital conducting innovative (medical) research. There are three focus areas in the scientific research at the JBH: nutrition and lifestyle, safety, and care chains. Key to the research conducted is multidisciplinary approaches focusing on improving care processes both from a logistical and medical point of view. Next to multi-disciplinary internal collaborations, the $\mathrm{JBH}$ researchers are involved in many projects with, among others, academic medical centers, insurers, and medical technological companies.

The research presented in this thesis contributes to bridging the gap between theory and practice, as we present a mixture between theoretic research and pragmatic implementation-oriented projects. The research is part-time performed at the Center for Healthcare Operations Improvement and Research (CHOIR) at the University of Twente and part-time at the JBH. From our experience the presence of the researcher at the hospital significantly contributes to the chances of successful implementation. The research contributes to the aims and core values of the JBH not only through the research presented in this thesis, but also through several advisory contributions of CHOIR's researchers to other JBH projects not presented in this thesis. The focus of each of these projects is improving efficiency and safety, organizing care from a patient-centered perspective, and providing timely care.

\subsection{Thesis outline}

This thesis aims to bridge the gap between theory and practice, as it displays research that focuses both on theoretical results and implementation-oriented case studies. Furthermore, we elaborate on the experienced problems with implementing research results, and provide several factors for successful im- 
plementation. The chapters are organized in five parts, each containing two chapters. Each part and chapter will be introduced briefly.

Part I focuses on OR methodologies applied to hospital wards and their integration into practice. Chapter 2 first provides an overview of the different performance measures and OR methodologies applied to hospital wards. Next, we review OR literature applied to hospital wards. Based on logistical characteristics and patient flow problems, we distinguish the following particular ward types: intensive care, acute medical units, obstetric wards, weekday wards, and general wards. We analyze typical trade-offs of performance measures for each ward type, and the common OR models applied to it. Additionally, we provide four modeling examples, discuss reported experiences with implementation of the research, and highlight voids in the literature that may be directions for future research. Chapter 3 present the results of a implementation-oriented case study on medical wards of the JBH that experienced unbalanced bed occupancies. Based on the results of both queueing theory and discrete event simulation the $\mathrm{JBH}$ implemented an intervention, which appeared to result in significant improvements.

Part II consists of two chapters on online appointment scheduling. In this thesis, online appointment scheduling refers to systems in which customers receive an immediate answer to their appointment request. This answer is either an appointment time, or a refusal. Chapter 4 provides an extensive literature review on online appointment scheduling for different application areas, not limited to healthcare. The literature is categorized according to the number of appointments each customer requires, the number of resource types at the facility, and the horizon at which the scheduling decisions are made. We provide an overview of the scheduling decisions, the objectives, and the operations research methods applied in different application areas. We identify similarities and differences between application areas and categories of our taxonomy, and highlight voids in the literature that represent opportunities for future research. In Chapter 5 we study the effect of introducing advance reservation, i.e., an appointment system instead of a walk-in system, on the blocking probability for a general queueing model. It appears that the effect of advance reservation may be positive or negative, depending on the system parameters. The lower blocking probabilities are achieved because the system with advance reservation tends to accept many relatively short jobs.

Part III entails two chapters in which resource schedules are optimized to improve patient flow for two JBH departments, both with the focus on practical relevance. In Chapter 6 we apply both queueing theory and discrete event simulation to the JBH Breast center to improve the schedule such that (1) the access time norms are met, and (2) patient waiting time is balanced with staff idle time and overtime. The implementation of the preliminary results appeared 
to significantly improve patient access times and the times between the first appointment and the diagnose. In Chapter 7 we present an implementationoriented case study, in which we investigate different appointment scheduling rules for the JBH plaster room. At the plaster room patients may either make an appointment or walk-in. Currently, the plaster room experiences strongly fluctuating patient waiting times and technician overtime. Invoking discrete event simulation, we investigate different appointment slot lengths and times, different proportions of patients making appointments instead of at their own preferred times, and different arrival rate scenarios.

Part IV focuses on optimizing medical doctor schedules, to improve compliance with both doctors' preferences and patients' access times norms. In Chapter 8 we optimize the schedule of gynecologists by developing an integer linear programming model. Gynecologists, and doctors in general, typically have many different tasks, such as seeing outpatients and visiting the ward. Each gynecologist can perform only a subset of the possible tasks, and there are many (soft) constraints on the sequence of tasks in a schedule. Since the time to solve the model is large, we investigate several heuristics, of which the local search heuristic appears to be very effective for practical purposes. Chapter 9 provides a quantification of the benefits of scheduling outpatient clinic hours dynamically, which implies that part of the capacity is only scheduled in case the access times exceed a certain threshold. The optimal dynamic scheduling policy is obtained through Markov decision theory, and compared to the optimal static schedule obtained invoking an integer linear program.

Part V contains two chapters on assigning treatment rooms at a typical emergency department (ED). Such departments often experience severe overcrowding, which may put patient lives at risk. Typically, doctors at EDs use multiple rooms in parallel; while one patient awaits test results in a treatment room, the doctor visits other patients. The assignment of rooms among the residents is often unbalanced, which affects the blocking probability and waiting and sojourn time of patients. In Chapter 10 we analyze patients' expected sojourn times invoking a queueing model in a random environment, for different room assignment policies and working routines of the doctors. We conduct a discrete event simulation to validate our model in case of time-varying arrivals, which are typical for EDs. In Chapter 11 we extend this approach by optimizing the decision which patient each doctor should treat next. To incorporate time-varying arrivals and treatment characteristics that depend on both the doctor and patient type, we invoke a mixed integer program in a rolling horizon approach.

Chapter 12 is the last chapter of this thesis and contains the conclusion and outlook. In this chapter we reflect on all chapters and our results. Additionally, we give an outlook on Operations Research in healthcare. 



\section{Part I}

\section{Operations Research applied to hospital wards}

Chapter 2

N.M. van de Vrugt, A.J. Schneider, M.E. Zonderland, D.A. Stanford, and R.J. Boucherie. Operations Research for Hospital Wards and its Integration into Practice. Submitted.

Chapter 3

S.M. Cornelissen, N.M. van de Vrugt, E.A.A. Smits, and T.P.J. Timmerhuis. Slim samenwerken aan een evenwichtige bedbezetting. [In Dutch] Submitted. 



\section{Operations Research for hospital wards and its integration into practice}

\section{$2.1 \quad$ Introduction}

During hospitalization, patients spend most of their time in wards. These wards are also referred to as inpatient care facilities, and provide care to hospitalized patients by offering a room, a bed and board. Wards are strongly interrelated with upstream hospital services such as the operating theater and the emergency department. Due to this interrelation it is essential to attain a high efficiency level at hospital wards in order to achieve efficient patient flow. Hospital ward management often aims for bed occupancy rates above $85 \%$ in order to maximize throughput, which leaves little slack for flow fluctuations, and results in refused, deferred and/or rescheduled patients.

Operations Research (OR) can give managerial insights about trade-offs between performance indicators, such as bed occupancy rates and deferred patients. Although OR methods have the potential to lead to large improvements in all sorts of processes, it appears that the cases in which the models and/or results have been actually implemented are sparse.

Our aim in this review chapter is to guide both researchers and healthcare professionals through the OR concepts that have been applied to hospital wards. We first present the terminology on the different types of wards and performance indicators covered in this chapter. Second, we briefly illustrate the most commonly applied OR models. Third, we give an overview of literature where OR techniques are applied to ward related problems, followed by some detailed examples on how to apply these models. We conclude the chapter by looking at the integration of OR models into practice and possibilities for further research.

\subsection{Hospital ward types and terminology}

In this section we introduce the types of hospitals wards as used in this chapter. The different types are distinguished based on the logistical characteristics of the wards, such as the available resources, typical in- and outflow, and typical patient flow related problems. Medically different wards may have similar logistical flow 
dynamics and may therefore be analyzed with similar OR models. Hereafter, we introduce the terminology used in the main performance indicators for wards.

Throughout this chapter, we define a hospital ward as follows: an area or unit within a hospital where inpatients with comparable medical conditions are admitted to a bed to receive care. This typically involves staying overnight until their medical condition changes in such a way that the patient either leaves the hospital, or is transferred to a ward with a different level of care. We therefore place the beds associated with the operating theater (OT), the emergency department (ED) or the outpatient clinics outside the scope of this chapter, as they usually temporarily accommodate patients that undergo a (short) treatment.

The logistical performance of wards is generally assessed by three indicators that are related to each other: throughput, blocking probability and occupancy. The exact definitions of these three performance indicators are given in Section 2.2.2, after we define the different ward types.

\subsubsection{Taxonomy}

In this chapter we classify relevant literature on wards based on the logistical characteristics of a ward: the type of in- and outflow, typical length of stay $(\mathrm{LoS})$ and resources, and planning problems the wards face. We distinguish the following types of wards:

- Intensive care unit (ICU)

- Acute medical unit (AMU)

- Obstetric ward (OBS)

- Weekday ward (WDW)

- General ward

We describe each type of ward in terms of logistical characteristics below and highlight differences and similarities between the types.

Intensive care unit (ICU) For this category in our taxonomy we group several ward types with similar logistical characteristics: traditional ICUs, specialized ICUs, and critical, high or medium care units. Specialized ICUs are, for example, stroke units, cardiac care units and neonatal ICUs. High care and medium care units, also referred to as step-down units, are wards that accommodate severely ill patients that are not ill enough for the ICU but are too ill to admit to a regular ward. Some hospitals combine both high and medium care beds into one ward, in the United Kingdom often referred to as critical care unit. Others distribute beds over two separate wards with different levels of care, or have certain beds labeled for high(er) care at each specialty's regular ward. The difference between high and medium care is generally the necessity of breathing support. In the remainder of this chapter we refer to the ward types discussed in this section as 'ICUs'.

The ICU of a hospital accommodates the most severely ill patients who require constant close monitoring and support from advanced medical equipment. Specially trained nurses take care of these patients on a 1:1 basis (except for 
medium care, where the ratio is 1:2), and intensivists visit the patients often. An ICU is highly equipped with mechanical ventilators to assist breathing, cardiac monitors, external pacemakers, dialysis equipment for renal problems, infusion pumps, and equipment for the constant monitoring of bodily functions. Most hospitals treating acute patients have an ICU, and some additionally form specialized ICUs. The medical equipment is then adjusted to the specific patient population.

Both acute and elective patients may be admitted to an ICU. Acute patients are transfered from the OT, ED or from a ward if they rapidly deteriorate. Elective patients are admitted immediately after a major surgery with high risk of complications. The LoS at an ICU is highly variable, and differs from the LoS at regular wards: patients either stay briefly (1-2 days) or for several weeks or months.

Due to the used equipment, high nurse-to-patient ratio and highly skilled staff, the ICU has the highest costs per bed of all hospital wards. An ICU preferably does not defer patients, as this would imply serious mortality risks. However, the costs per bed do not allow for a large buffer in the number of available beds. Therefore, ICUs tend to be fully occupied most of the time, and discharge the least ill patient when a bed needs to be freed for a newly arriving patient, or cancel an elective procedure at the OT which requires ICU capacity afterwards. The size of an ICU is naturally defined by the number of arriving patients and their LoS, but also partly by national care customs: in the United States in the year 2009, the number of ICU/critical care beds per 100,000 head of population was 25.3 [486, 511], compared to an average of 11.5 in Europe in 2011, where Germany has the highest with 29.2 beds, and Portugal the lowest with 4.2 beds per 100,000 head of population [415]. The differences are partly because different countries may have different definitions of ICU, critical care, high care and medium care beds [415], but could also reflect differences in available resources.

Acute Medical Unit (AMU) AMUs lack a uniform definition. Scott et al. [439] use the following: 'an AMU is a designated hospital ward specifically staffed and equipped to receive medical inpatients presenting with acute medical illness from EDs and outpatient clinics for expedited multidisciplinary and medical specialist assessment, care and treatment for up to a designated period (typically between 24 and 72 hours) prior to discharge or transfer to medical wards'. AMUs are also known under synonyms as 'emergency observation and assessment ward', 'acute assessment unit' and 'acute medical assessment units'. The review papers by Cooke et al. [122] and Scott et al. [439] provide a comprehensive overview of definitions and concepts for AMUs.

Hospitals that are frequently faced with ED congestion, often consider creating an AMU. The goals of such a department can vary, but usually include several of the following criteria: to rapidly assess acute patients who don't have a life-threatening medical condition, to observe patients with fairly predictable 
conditions that require close monitoring, and to alleviate pressure from and increase throughput in the ED. The inflow of patients to AMUs originates from the ED, and from urgent outpatient clinic or general practitioner referrals.

AMUs differ in the patient types they admit; for example, a hospital can decide to only admit medical patients [439]. Patients who require intensive or high care are usually immediately accommodated at the ICU or specialized wards [122, 439]. At an AMU, patients are temporarily (less than 24 hours, or 24-72 hours) hospitalized until: (1) a bed at an inpatient ward becomes available or (2) discharge. ED patients who have to wait for test results or certain medication to work, or patients who require observation for a short period of time can also be admitted. There are typically no elective admissions to AMUs, and the number of patients admitted and discharged per day is high compared to other wards.

Specially trained doctors will be present more frequently at an AMU compared to the standard once or twice a day at general wards, to ensure a short LoS and adequate quality of care. A hospital can also decide that multiple doctors of different specialties should visit the AMU frequently. The nurses working at an AMU have multidisciplinary skills. Regarding other resources, such as technical equipment, an AMU is comparable to a regular ward. All in all, an AMU bed costs more than a bed at a regular ward.

Several reviews exist that asses the clinical effectiveness of AMUs [71, 122, 132, 439]: patients may for example perceive fewer ED waits longer than 4 hours, reduced LoS by earlier senior doctor consultation, a reduced risk of inappropriate discharge, and a reduced risk of being accommodated at different wards than the medically preferred ward. Staff satisfaction may increase, as at general wards the process is disturbed less, fewer patients need to be admitted during night-time, and ED workload is decreased. For an AMU to be cost-effective, the scale should be large enough to cope with varying patient arrival patterns. Often, AMUs serve as a buffer for both the ED and inpatient wards. Since an AMU treats only urgent patients and should alleviate ED congestion, management is more focused on throughput and LoS. The target utilization of the AMU beds is therefore typically lower than at a general ward.

Weekday ward (WDW) WDWs are wards admitting patients with an expected LoS between two and five days, which are usually only open on weekdays. WDWs provide a similar or slightly lower level of medical care compared to regular wards. WDW-type of hospitals are also sometimes referred to as 'Monday to Friday clinic' or 'week hospital'.

Generally, most patients at WDWs are elective, and can be transferred to regular wards without any health risks. Only patients with a highly predictable LoS may be admitted, which is why WDWs mostly treat patients for which strict treatment protocols apply. Scheduling patients at a WDW is complicated since each patient has a different LoS and a certain urgency level, which implies a deadline by which the patient should be treated. The requirement that the ward 
should be closed during weekends also complicates patient scheduling.

Nurse to patient ratios are usually 1:5 or 1:6, which are comparable to regular ward ratios. Nurses are skilled to care for a broad spectrum of medical conditions, so not the treatment itself makes this unit complex, but the different types of patients treated at the same time. The resources available at WDWs are comparable to those at regular wards. Due to the brief hospitalization of the patients, WDWs admit and discharge relatively many patients each day, and the beds require cleaning more often than at regular wards. The costs per bed are relatively low, because the ward is closed during weekends.

Since all patient admissions are elective and patients' LoS is predictable before admission, the bed census at a WDW can be predicted quite accurately. This implies that high occupancy rates can be achieved, so target utilizations are typically set higher than for general wards.

Obstetric ward (OBS) Obstetric and gynecology wards provide care for women during their pregnancy, during and after labor, and also take care of their newborns. Additionally, gynecology wards accommodate women with problems regarding their reproductive organs. The women at these wards often require (brief) surgical intervention, and typically a short hospitalization. Some hospitals group these types of wards under names like 'birthing center', 'maternity clinic', or 'women's and child's center'.

Many of the patients are urgent, and patient arrivals are mostly determined by external factors, e.g., by how many pregnant women live in the surroundings of the hospital. Relatively many patients are admitted during night time. In case of bed shortage it is possible to accommodate patients in different wards (for example, at a WDW) but this requires some consideration, as women treated for infertility should not be placed among newborns. Therefore, sizing OBSs and nursing teams is a challenging task, and given the nature of the admissions (mostly unscheduled), it requires different OR analyses compared to general wards.

The nurse to patient ratios may be slightly higher compared to general wards: 1:4 to 1:6 (excluding newborns). Target utilization levels are often lower than for general wards, as a relatively large proportion of the patients is acute. Resources at obstetric wards are comparable to general wards, with the addition of bassinets. Hospitals tend to pay more attention to the wards' decoration, and to safety guidelines, e.g., to avoid newborns going home with the wrong parents.

General wards General wards in hospitals are often dedicated to a single medical specialty such as neurology, geriatrics, or hematology. As these wards are generally equipped with similar resources, we aggregate these ward types. General wards can either be surgical or medical; surgical wards are specialized in nursing patients from surgical specialties, and medical wards accommodate patients from the other medical specialties. Some wards, such as psychiatric or geriatric wards, are closed, implying that patients cannot leave the wards without approval of a nurse. Some wards are equipped with a specific type of resource, 
such as dialysis machines and heart monitors. Patients with a particular medical specialty are typically not all accommodated in the same ward, but may also be admitted at for example a WDW or an ICU.

Patient admissions can be acute or elective, and patients can be admitted to the ward directly, or transferred from a ward with a higher care level. If patients cannot be admitted to their designated ward, there is a list for each possible diagnosis with alternative wards where the patient may also be accommodated. These so-called 'boarder' patients are sometimes transfered back to their designated ward if a bed becomes available, but this policy differs among hospitals.

Some wards dedicated to a single specialty typically have patients with long LoS, such as hematology, or with short LoS, such as ophthalmology. Dialysis wards treat many elective patients requiring repetitive care, taking several hours on multiple days each week for a long period of time.

The nurse-to-patient ratio at general wards is typically 1:5 to 1:6. Each ward usually employs a mixture of highly educated nurses and supporting staff (who, for example, help in washing patients). A ward may have resources like infusion pumps, medication or specialty dependent equipment. The cost per bed ratio is relatively low, and the target occupancy rate for most general wards is about $85 \%$.

\subsubsection{Terminology}

In healthcare a concept such as 'occupancy', which may seem simple at first sight, has several different definitions. Therefore, we define all concepts used further on in this chapter in the following paragraphs.

Each ward has a certain capacity, which is expressed in terms of the number of patients and their aggregated care intensity that the ward can accommodate. The capacity of a ward is measured by the number of beds and nurses, and there are different types of capacity. The physical capacity is the number of beds at the ward. Each nurse can take care of a certain number of patients in parallel (determined by the nurse-to-patient ratio), which determines the structural available capacity. Additionally, temporary capacity changes can occur; for example bed closures in holiday periods, or beds that are used which are officially not staffed in case of bed shortage. The structural capacity and temporary changes together determine the (average) realized available capacity.

Suppose, in a highly stylized example, that a hospital ward has 15 beds in a certain area. During daytime three nurses are scheduled to work at the ward, and each nurse can take care of at most four patients at the same time. If a nurse is ill, another nurse is called in for assistance, so the number of nurses at the ward during daytime hours will always be equal to three. During evening and night shifts possibly different numbers of nurses work at the ward but, for the ease of 
this example, let us assume that those numbers of nurses are not restricting the number of patients the ward can accommodate.

Each summer and Christmas holidays the ward experiences decreasing patient numbers, and decides to only schedule two nurses for the day shifts. The holiday periods together last eight weeks. Then, for this ward the physical capacity is 15 beds, and the structural capacity is $3 \times 4=12$ beds. Due to the holidays, each year has eight weeks in which only eight beds are open, so the average realized capacity is:

$$
\frac{8 \times 8+(52-8) \times 12}{52} \approx 11.4 \text { beds. }
$$

As mentioned in the introduction of this section, the logistical performance of a ward is assessed by three performance indicators: throughput, blocking probability and occupancy. These indicators are all related to each other. The throughput of a ward can be measured as the number of admissions or discharges per time unit. The blocking probability of a ward is the percentage of patients that request a bed at the ward at an instance that there are no available beds:

$$
P^{b}=\frac{\text { No. patients not accommodated at ward }}{\text { Total no. patients requesting a bed at ward }} \times 100 \% \text {. }
$$

Blocked patients are either accommodated in a different ward, or deferred to another hospital. There are many definitions of bed occupancy, on which we will elaborate below.

In contrast to throughput and blocking probability, bed occupancy can be quantified by three definitions: based a on bed census at certain time, based on real LoS or based on the number of hospitalization days. Here we aim to give an overview of the most commonly used definitions. One of the definitions of bed occupancy includes the bed census measured once a day at a specified point in time. A hospital can for example determine a ward's bed census every morning at 10:00, take the average of these measurements, and divide this by the structural available capacity to determine the bed occupancy. Then:

$$
O_{b c}(t)=\frac{\text { average bed census at time } t}{\text { structural available capacity }} \times 100 \% \text {. }
$$

Note that for the occupancy it also matters how the capacity of a ward is calculated; in most hospitals the structural available capacity is used. A slightly different way of expressing the occupancy of a ward is obtained by taking the average of multiple bed census measurements taken throughout each day, for example each hour, instead of once each day. The advantage of taking more measurements is that it will better reflect actual bed usage. 
Hospitals may also define the occupancy of a ward as the ratio between the total time patients were in beds at the ward and the total time available:

$$
O_{L o S}(T)=\frac{\text { sum of all LoSs for all patients in time period } T}{\text { structural available capacity } \times \text { time period } T} \times 100 \% \text {. }
$$

This measure is calculated using admittance and discharge time stamps for a certain measurement period, or by multiplying the average LoS with the number of patients accommodated at the ward. This occupancy measure reflects the actual time the beds are used. However, after each patient a bed requires cleaning and is therefore not directly available for admitting new patients.

Until recently, it was common in Dutch hospitals to determine the bed occupancy using the hospitalization days declared to the insurance companies:

$$
O_{h d}(T)=\frac{\text { sum of hospitalization days for all patients in } T}{\text { structural available capacity } \times \text { length } T} \times 100 \% \text {. }
$$

Financial hospitalization days were counted in integers, and could be declared if the patient was in a bed before 20:00 and discharged after 7:00 the next day. This implied that the occupancy could be over 100\% as beds can be reused if patients are discharged early in the day and new patients are admitted in the afternoon. A drawback of this measure is that it cannot be used as a targeted occupancy for all ward types. Such a situation would arise in wards in which patients generally stay for only a part of a day so that multiple patients can be served by the same bed on the same day (e.g. gynecology). In this system, these wards should therefore achieve occupancy targets over $100 \%$, while wards at which patients stay much longer (e.g. geriatrics) will suffer severe bed shortages if the occupancy is over $90 \%$.

This is an example of an arrival and discharge process at a ward, in order to illustrate the different concepts of occupancy. Consider a ward with three beds that is empty at the start of our observation period. We choose to observe the ward from 8:00 on day 1, until 17:00 on day 4. In this period the following

\begin{tabular}{|c|c|c|c|c|c|c|}
\hline & \multicolumn{2}{|c|}{ Arrival } & \multicolumn{2}{|c|}{ Discharge } & \multirow[b]{2}{*}{ LoS } & \multirow[b]{2}{*}{ Hosp. days } \\
\hline & Day & Time & Day & Time & & \\
\hline Patient 1 & 1 & 8:00 & 2 & 18:00 & 1.42 & 2 \\
\hline Patient 2 & 1 & $10: 00$ & 4 & 8:00 & 2.92 & 4 \\
\hline Patient 3 & 1 & $15: 00$ & 2 & $8: 00$ & 0.71 & 2 \\
\hline Patient 4 & 2 & $3: 00$ & Patie & is blocked & - & - \\
\hline Patient 5 & 2 & 9:00 & 3 & 8:00 & 0.96 & 2 \\
\hline Patient 6 & 3 & 9:00 & & r day 4 & 1.33 & 2 \\
\hline Patient 7 & 4 & 10:00 & & $\mathrm{r}$ day 4 & 0.29 & 1 \\
\hline
\end{tabular}
patients arrive:

Note that the LoS for patients 6 and 7 in the table is not their exact LoS but only the part until the end of the observation period. The bed census for this ward 
is depicted in Figure 2.1. The blocking probability for this time period equals $1 / 7 \approx 15 \%$. The different occupancy measures are calculated as follows.

The bed census at 10:00 for day 1 to 4 is $2,3,2$, and 1 , respectively, so the average equals 2 . Therefore $O_{b c}(10: 00)$ (the occupancy based on the 10:00 census) equals $2 / 3 \approx 66.7 \%$. The average hourly bed census is 2.2 , so the occupancy based on the hourly bed census equals $2.2 / 3 \approx 74.8 \%$.

The sum of the LoS for all patients at this ward in this observation period, $T$, equals 7.63 days. The length of the observation period is 3.38 days. Therefore, $O_{L o S}(T)=7.63 /(3 \times 3.38) \approx 75.3 \%$.

The sum of the hospitalization days declared for these patients is 13 , and the total number of days in this observation period is four. Therefore, $O_{h d}(T)=$ $13 /(3 \times 4) \approx 108.3 \%$.

Figure 2.1 Bed census for example.

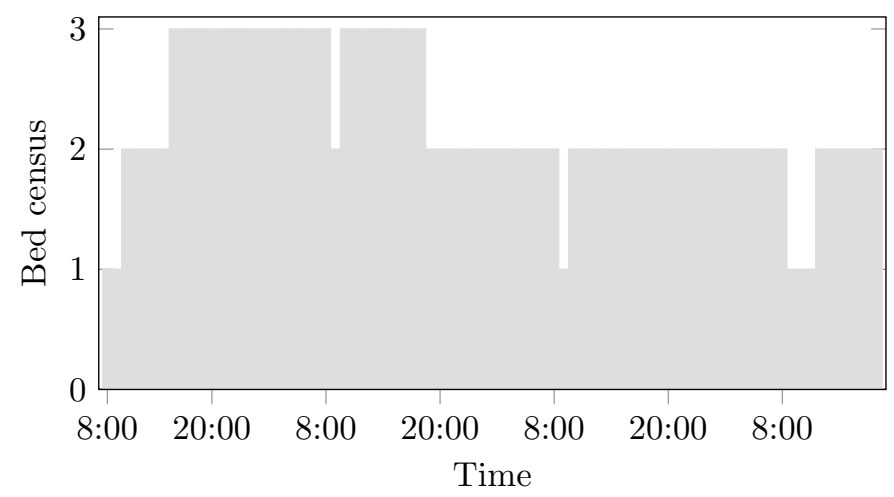

The occupancy measure with hospitalization days is always higher than the other occupancy measures. The ordering of the remaining concepts of occupancy depends on the ward studied.

Hospital management determines which of the aforementioned occupancy measures is used, and sets the targets for the desired performance measures for each ward separately. The trade-off between occupancy and waiting time or blocking probability was established as early as 1952 by Bailey [26]. A high occupancy usually results in a high blocking probability. Therefore it is important for management to balance these three performance indicators. Adequate targets for the performance indicators depend on many factors, for example: the capacity of a ward, the fraction of admissions that is acute, the possibility of deferring admissions, the cost per bed, and the ward layout. Large wards have economies of scale, so a higher bed occupancy can be achieved with a lower blocking probability. Therefore, the occupancy target of a large ward can be higher than that of a relatively small ward. If a ward has mostly acute admissions, occupancy tar- 
gets need to be set lower; elective admissions can be rescheduled in case of bed shortage, while acute admissions cannot. If the deferral of an arriving patient could give rise to life threatening situations, e.g., in case of an ICU, a ward has to lower the target occupancy to achieve a lower blocking probability. However, such wards usually have high costs per staffed bed, driving the occupancy targets upwards. Finally, if a ward has many rooms with multiple beds, the bed assignment is less flexible compared to wards with many single bed rooms. If, for example, a patient has an infectious disease he cannot share a room with others. Concluding, it can be said that determining adequate occupancy, blocking probability and throughput targets is a challenging task.

\subsection{OR model types}

In this section we elaborate on the more commonly used OR models. The model categories are based on ones applied in the ORchestra database [241]: algorithms, mathematical programming, dynamic programming, regression, time series, Markov models, stochastic models, queueing theory, and simulation. We define each of the OR techniques, and provide introductory examples. We conclude this section by highlighting the potential for OR models to aid decision making in healthcare problems.

\subsubsection{Overview of OR models}

In this section we describe the more commonly used OR models in the context of a hospital ward setting: whereas OR researchers address 'servers' we use the term 'beds', and the 'customers' are referred to as 'patients'.

Algorithms Any procedure that follows predefined steps may be called an algorithm. Algorithms are often used for solving optimization problems, and are either based upon an exact mathematical analysis, or upon some heuristic rationale. Exact algorithms return an optimal solution but have significant long runtime, while heuristics approximate the optimal solution in order to decrease the runtime of finding a solution.

Algorithms are often applied to scheduling problems. The most simple illustration of a scheduling heuristic is the 'greedy algorithm', which prescribes that we schedule every patient at the earliest available bed or appointment slot. The 'earliest due date first' heuristic schedules the patients from the waiting list at the first available resource according to ascending maximum access times. Exact algorithms are typically more complicated than heuristics, so heuristics are often preferred for practical implementations. For more information on scheduling algorithms, the reader is referred to [401].

Mathematical programming Mathematical programming is the name given to a variety of related fields with a common form: the optimization of one or more 
objectives subject to a set of limitations, called constraints. These fields include linear and non-linear programming, integer programming, stochastic programming, and network flow problems. The most commonly used of these is the field of linear programming, which can be stated as follows. One seeks to optimize (that is, maximize or minimize) a single objective, which is a linear function of a vector $\mathbf{x}$ of decision variables (that is, variables whose values we have some control over). The solution space of $\mathbf{x}$ is subject to a series of linear constraints, which state the operational limitations under which the system must operate. In matrix form, a linear program to maximize the objective can be stated as:

$$
\begin{aligned}
& \max z=\mathbf{c x} \\
& \text { subject to: } \mathbf{A x} \geq \mathbf{b} \\
& \mathrm{x} \geq 0 \text {. }
\end{aligned}
$$

Here, $\mathbf{c}$ is a row vector containing the reward rates per unit increase in a particular decision variable, $\mathbf{A}$ is the matrix whose rows contain the coefficients for the decision variables in the various constraints, and $\mathbf{b}$ is the column vector of right hand sides representing the limits for these various constraints.

Such a formulation is called linear, as the objective function and the constraints are all linear functions of the decision variables. Likewise, a mathematical program can be 'integer' if some or all of the decision variables can only take integer values, or 'stochastic' if some or all of the variables are random in nature.

A more practical example of this model is given in Section 2.5.4. For more information, see [519]. A related yet distinct area frequently used in healthcare applications is the field of dynamic programming, which we consider next.

Dynamic programming All sequential decision making problems are aggregated in the dynamic programming category. This type of models break the overall decision problem into a series of more easily solved sequential problems, consisting of the different phases at which a decision maker should choose one of the available actions. In each phase the 'system' under consideration is in a certain state, where the state contains enough information to decide which action would result in the best possible outcome for the system. The chosen action may result in direct costs, and determines the state of the system in the next phase, either with certainty or known likelihood. This can be stated more formally as follows: denote the phases by $t$, the states by $i$, the possible actions by $a$, the direct costs associated with action $a$ when in state $i$ by $c(i, a)$, the probability to go from state $i$ to $j$ when action $a$ is chosen by $p(i, j \mid a)$, and the value function by $V_{n}(i)$. A dynamic programming model may minimize costs, or maximize rewards. A dynamic programming model (here stated with the first objective) is optimized backwards by the recursion:

$$
V_{n}(i)=\min _{a}\left\{c(i, a)+\sum_{j} p(j, i \mid a) V_{n+1}(j)\right\} .
$$


Markov decision models are related to dynamic programming models. However, whereas dynamic programming works backwards in time (from phase $n+1$ to $n$ ), Markov decision problems are solved forwards in time (from phase $n$ to $n+1)$. Dynamic programming models are therefore more suitable for problems with a given deadline, where Markov decision theory is often applied to problems with infinite horizon. For more information, see [519].

Consider the following illustration: suppose we should schedule the admissions of patients $1,2, \ldots, 20$ with similar diagnoses at a ward for the upcoming five days. Each patient stays for at least one day, and $30 \%$ of the patients need to stay an additional day. Each day we should decide which patients to admit, such that the probability that there are enough beds is above a certain threshold and all patients are admitted after five days. Then, the phases of the system are the days $1,2, \ldots, 5$, the state of the system is the number of free beds, and our action is the number of patients we schedule on the current day.

Regression and time series Forecasting methods are used to forecast future values of a certain variable (or variables) based on historical data. Time series models such as 'moving average' and 'exponential smoothing' take a certain number of measurements as input for the forecast. Suppose we want to estimate $x_{t}$, the average occupancy of a ward on day $t$. We have data on the average occupancy for each day $1,2, \ldots, t-1$. The moving average model is given by:

$$
x_{t}=\frac{\sum_{i=t-N}^{t-1} x_{i}}{N},
$$

with $N$ the number of days used to calculate the moving average, to be determined by the user. Exponential smoothing is used if the variable fluctuates around a base level. Let $A_{t}$ be the smoothed average of the average occupancy at day $t$, then:

$$
A_{t}=\alpha x_{t}+(1-\alpha) A_{t-1}
$$

with $A_{0}=x_{0}$ as starting value, and $0<\alpha<1$ the smoothing factor. Each new measurement of the occupancy is added to the forecast, but to smooth out strong fluctuations the factor $\alpha$ is included.

Regression analysis estimates the relationship between the dependent variable that we wish to forecast, $x_{t}$, and (multiple) independent variables. The linear regression model is the most simple, and is described by:

$$
x_{t}=\beta_{0}+\beta_{1} y_{t}+\epsilon_{t} .
$$

Here, $y_{t}$ is the independent variable, $\beta_{0}, \beta_{1}$ are coefficients that set the relationship between $x$ and $y$, and $\epsilon_{t}$ is an error term. For example, we could try a forecast with $y_{t}$ different for each day of the week. The coefficients $\beta_{0}$ and $\beta_{1}$ should be estimated to best fit the historic occupancy, and may be determined through the least squares method. 
Statistics packages such as $\operatorname{SPSS}^{\circledR}$ and Minitab ${ }^{\circledR}$ contain most forecasting tools, and also Microsoft Excel ${ }^{\circledR}$ contains formulas for forecasting. For more information, see [216, 519].

Markov and stochastic models A stochastic model is a description of the relation between random variables, whose values are not known with certainty beforehand. A random variable measured at discrete time points, e.g. each day at 10:00, is called a discrete-time random variable. A continuous time random variable is measured continuously, for example a patient's heart rate or temperature.

Markov models are a specific type of stochastic model, and have the property that the next value in the stochastic process is independent of its past, given its current value. An example of a Markov model is the outcome of a coin toss. We use the term 'stochastic model' for all stochastic models that do not have this property and do not fall into one of the other model categories. The reader is referred to $[423,519]$ for more information.

Queueing theory Queueing theory is the study of waiting lines in production systems. These systems consist of a waiting line and one or multiple servers, and are defined by an arrival and service process, see Figure 2.2.

Figure 2.2 A simple queue.

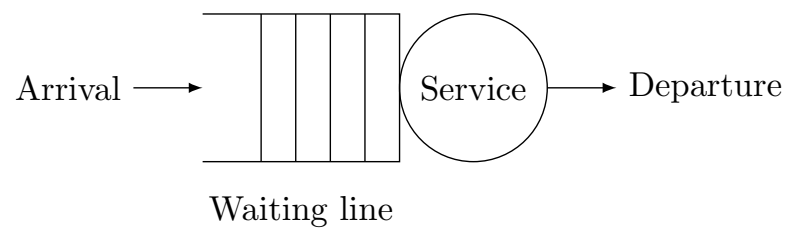

A short way of referring to queues is by Kendall's notation: $A / B / c(/ c+k)$, where $A$ and $B$ denote the arrival and service process, respectively, $c$ is the number of servers, and $k$ is an optional argument that denotes the number of places in the waiting line if this number is limited. Most queueing models assume Poisson arrivals, for which $A=M$. The service time distribution may be deterministic $(D)$, exponential $(E)$ or general $(G)$. Typical performance measures that may be evaluated using queueing models are blocking probabilities, occupancy, throughput, patient waiting times, and bed idle times. Section 2.5 contains several examples of queueing models.

The QTS tool developed by Gross et al. [203] is convenient for obtaining performance measures for most queueing (network) models with homogeneous arrival and service rates. For additional basic information on the queueing models described in this section the reader is referred to [519, 549].

Simulation Simulation models are used to mimic the evolution of a system over time, and consist of a list of what-if rules and procedures. We distinguish among discrete event simulation, Monte Carlo simulation, and system dynamics models. 
Discrete event simulations are event-driven routines, in which an eventlist is kept that contains the time stamps and types of events that will occur on those time stamps. With Monte Carlo simulation, repeated sampling from a probability distribution is carried out to obtain information on relevant performance measures. System dynamics models focus on the way different entities of the model influence each other, which relations are captured in a system of coupled, often non-linear differential equations. For more information on simulation models, see $[294,519]$.

Different simulation software packages exist, with different requirements regarding the user's programming abilities. Graphical simulation tools can often support the model validation process as the practitioners can see how the patients for example walk through the clinic. A drawback of graphical simulation models is that computation speed is reduced compared to non-graphical simulation packages.

\subsubsection{The potential for OR models to aid decision making}

The advantage of OR models is that possible interventions can be evaluated in a safe environment, reducing the risk of implementing an intervention that appears to be counter-productive. OR models may be invoked for different objectives, for example to provide insights or to optimize a certain performance measure. In this section we provide an overview of the most common objectives. Recall from Section 2.2 that the logistically important performance measures for hospital wards are throughput, blocking probability and occupancy.

OR models provide insights in one or more of these performance measures, and are often used to investigate the effects of changes in the number of arriving patients and/or capacity. A possible objective in this area could be to determine the optimal capacity, warranting a prespecified maximal blocking probability and minimal occupancy levels.

A different objective would be to evaluate possible changes in the processes or policies of wards, for example the impact of opening an AMU, or by discharging patients earlier to wards with a lower care level (e.g., from ICU to a general ward). This objective may relate to multiple, interrelated wards. Possible interventions are often proposed by the practitioners, or they play a large role in checking the feasibility of the interventions.

Optimizing ward occupancy may also be addressed invoking OR models. Possible objectives include balancing the occupancy among different wards, maximizing the occupancy or throughput, and achieving a certain target occupancy. The occupancy may be improved by optimizing the patient admission policy, elective patients' admissions, or the schedule of the operating theater.

Related topics, not covered in this chapter, are for example optimizing the assignment of patients to beds, and optimizing patients' access times. The bed assignment problem becomes important when, for example, a ward accommodates patients with infectious diseases, or patients that do not share rooms with the opposite sex. A patient's access time is the number of days between the re- 
Table 2.1 Literature categorized by applied models and ward type.

\begin{tabular}{lcccccc}
\hline OR model & IC & AMU & OBS & WDW & General & Total \\
\hline Algorithms & 1 & 0 & 0 & 0 & 3 & 4 \\
Dynamic programming & 1 & 0 & 0 & 1 & 0 & 2 \\
Markov processes & 4 & 0 & 2 & 0 & 11 & 17 \\
Mathematical programming & 4 & 2 & 3 & 1 & 6 & 16 \\
Queueing theory & 13 & 2 & 3 & 0 & 17 & 37 \\
Regression & 1 & 0 & 0 & 0 & 1 & 2 \\
Simulation & 22 & 1 & 2 & 0 & 21 & 46 \\
Stochastic models & 1 & 1 & 0 & 0 & 3 & 5 \\
Time series & 1 & 0 & 0 & 0 & 2 & 3 \\
\hline Total & 35 & 4 & 6 & 2 & 48 & 95 \\
\hline
\end{tabular}

quest for an appointment (e.g., a surgery or diagnostic test) and the appointment itself. Patients' access times may be improved by optimizing patient admission schedules and/or the operating theater schedule. Additionally, material logistics and facility design problems may be interesting applications of OR models, but these applications are outside the scope of this chapter.

\subsection{Ward-related OR models}

In the previous sections we distinguished different ward types and their logistical similarities and differences, and different OR techniques. In this section we will review the OR literature for each ward type, emphasizing the main questions or problems the literature tries to solve, the context of problems (e.g., ward type) and the type of models invoked for each paper. In Table 2.1 the number of papers found for each ward type and OR model/method is displayed. If a paper invoked multiple OR models, we categorized this paper in all applicable categories.

\subsubsection{Intensive care unit}

At the ICU both elective and emergency patients arrive. Emergency patients mostly come from the ED or surrounding hospitals and elective patients mainly arrive after surgery. Since significant costs are involved, management tends to maximize utilization. This results in an increasing number of refusals and/or severe ill patients being transferred from the ICU to high, medium or regular care wards which could lead to situations where quality of care is at stake, and possibly to disruptions in the operating theater schedule. These are also the main problems the literature of this section focuses on: admission and discharge control.

Blair and Lawrence [49] use a queueing model $(M / G / c / c$ queue) to analyze the total minimally required number of ICU beds for burn care for the state of New York. The authors start by finding the number of beds at an aggregate level given a maximum blocking probability of $5 \%$, and then apply a heuristic allocating these ICU beds among several regional units, while trying to maintain the stated level of blocking. Litvak et al. [315] extend this $M / G / c / c$ queue to analyze an overflow model. Each ICU in this study reserves bed capacity for regional 
Table 2.2 Literature on ICUs categorized by applied models.

\begin{tabular}{ll}
\hline OR model & References \\
\hline Algorithms & {$[49]$} \\
Dynamic programming & {$[90]$} \\
Markov processes & {$[49,67,144,181]$} \\
Mathematical programming & {$[273,339,340]$} \\
Queueing theory & {$[49,68,69,141,201,267,315,340,356,449,518,528$,} \\
& $549]$ \\
Regression & {$[339]$} \\
Simulation & {$[58,59,124,134,267,268,273,277,315,339,340,343$,} \\
& $345,348,364,373,416,445,453,481,528]$ \\
Time series & {$[181]$} \\
\hline
\end{tabular}

emergency patients, which may be used as overflow beds in a certain region. To approximate the blocking probability of this overflow model, the 'equivalent random method' is used, while a simulation model is used to validate the results of this queueing model with historical data. The equivalent random method approximates the original network by truncating an infinite server network. A modified $M / M / c / c$ model is used by Shmueli et al. [449] to analyze different admission policies and their relation to survival gains. The policies consisted of: (i) the standard first come first served discipline; (ii) arrivals are served if and only if a bed is available and the survival gain is greater than an arbitrary threshold value; and (iii) arrivals are served if and only if a bed is available and the the survival gain threshold value is met, where in this policy the threshold value depends on the number of beds available. (If fewer beds are available, the threshold value for survival gain will increase.) The results show significant increase in survival gain in both the second and third policy compared to the first policy. The third policy showed only marginal survival gain compared to the second policy, while the number of rejected patients increased significantly. Also McManus et al. [356] use an $M / M / c / c$ queue for analyzing an ICU. The model is validated with observed data and it is shown that the calculated blocking probablities from the queueing model were accurate.

Next to queueing, discrete time Markov chains are also applied to ICUs. Dobson et al. [144] develop a Markov chain in order to analyze so called bumping; patient transfers from the ICU to free capacity for new arrivals which are more severely ill. Chan et al. [90] apply this technique analyzing the effect of ICU discharge strategies and bed census on patient mortality and total readmission load (patients that are hospitalized shortly after their last admission for the same medical condition).

Simulation is also applied often to analyze the required number of ICU beds. In $[273,345,416]$ this technique is applied to analyze several scenarios, for instance reserving ICU beds for emergency arrivals. Kim et al. [267] simulate several ICU arrival processes and compare these results with theoretical results using an $M / M / c$ queue. Based on the simulation model, the authors also determine the blocking probability for the current capacity. Another study by Kim 
et al. [268] analyzes several scenarios to minimize the number of elective surgery patients refused at the ICU. The efficient frontier method is used to plot the trade-off between the number of canceled surgeries and the average waiting time per scenario.

Mallor and Azcarate [339] combine several OR techniques to analyze the ICU: first, a regression model is proposed for modeling the ICU LoS; second, a comprehensive simulation model is developed for analyzing system behavior and blocking probabilities; and last mathematical programming is used to model the triage problem (which current and arriving patients require ICU capacity the most?) for early or delayed discharges from the ICU depending on high or low utilization of ICU capacity.

When analyzing patient logistics at the ICU, there is a clear distinction between the type of models used and the type of problems solved. Because a significant part of the arrivals at the ICU is unscheduled, queueing theory gives accurate and representative results. This technique is typically used to achieve general insights about blocking probability, occupancy, ICU capacity, and their mutual trade-offs. Markov chains are used to analyze bed census probabilities and the probability of bumping. Simulation is generally used to analyze multiple scenarios where particular details are involved and/or case-specific dynamics need to be studied.

\subsubsection{Acute medical unit}

The reviews on AMUs mentioned in Section 2.2.1 conclude that AMUs may have many advantages, but also that the evidence of economic effectiveness is thin. Cooke et al. [122] conclude that AMU 'performance is dependent on good management and availability of diagnostic services', and asserted that there is no proof of cost-effectiveness of AMUs. Scott et al. [439] provide an extensive list of success factors for AMUs. From an OR perspective, if a hospital does not add beds or staff to its current capacity for opening an AMU, the improved performance reported in the reviews is disputable. The beds assigned to the AMU are taken from other wards, decreasing the benefits of economies of scale and affecting other patients at those wards, and additionally, patients that require inpatient care after their stay at the AMU encounter more process steps than if they would have been admitted directly. Therefore, the effects of opening an AMU cannot be predicted beforehand without the use of appropriate mathematical models. Perhaps partly since AMUs are a relatively new concept, the OR literature with an AMU application is somewhat sparse. In this section, we review this available literature.

Depending on the performance measures of interest and research goals, several models could be applied to AMUs. We describe a goal programming approach used to minimize the delay from ED to AMU, and two different queueing networks to evaluate blocking probability and bed census.

Oddoye et al. [377] use a goal programming approach to determine the required additional resources (beds, doctors and nurses) for each hour of the day 
to minimize the delay patients experience on an AMU staffed with eight beds, two nurses and three doctors. Goal programming is an extension to mathematical programming, in which for each, typically conflicting, objective a target (or goal) is set and deviations from these targets are minimized. In the model, each patient requires a bed, and a specific treatment by a nurse, doctor, or both. A patient is delayed if there are no beds available upon arrival, or if the doctors and nurses are seeing other patients at the moment the patient requires care. For the case studied, the average LoS is five hours, and the run time of the model equals a day and a half. The conclusion is that only two doctors are required, and a third nurse should be standby in the afternoon and at midnight to cope with peak demand.

In a follow-up study for a bigger AMU (currently 58 beds), Oddoye et al. [378] simulate 14 scenarios with different numbers of beds. In their simulation, each resource type (beds, nurses, and doctors) has its own queue, and patients wait in these queues until the resource they require is available. Initial targets for each queue length are fed into a goal programming model, together with targets for total LoS and the number of beds. The authors minimize weighted positive deviations from these targets. The model output comprises the resource levels that minimize patients' delay at the AMU, and a trade-off between economic objectives, i.e., higher utilization of resources, and patient- and staff-related objectives is provided.

Utley et al. [487] study a network with one AMU and an aggregated regular ward, in which patients are transfered between the wards if their care requirements change. The authors use an infinite server queueing network to determine the probability that the bed occupancy on either ward exceeds a certain number of beds. Based on this probability, they determine the optimal assignment of the available beds to either the AMU or the regular ward. In case the total mean bed occupancy is $85 \%$, and $91 \%$ of the patients require acute care, they conclude that $60-65 \%$ of the available beds should be designated for acute care.

For a network comprising an ED, two aggregated wards, and an AMU, Zonderland et al. [552] determine the blocking probability by invoking a network of Erlang loss queues in which the AMU both has direct patient arrivals and serves as an overflow ward. They consider both urgent patients (arriving from the ED) and elective patients. The hospital is only allowed to reallocate existing beds from the wards to the AMU in determining the best AMU size, there are no additional beds available. The equivalent random method is used to analyze the network with overflows, since overflow traffic is not Poisson. The authors conclude that opening an AMU is beneficial for accommodating urgent patients, but the blocking probability for elective patients increases significantly.

The advantage of a simulation or goal programming approach over queueing networks, is that time-dependent arrivals can be incorporated relatively easy. However, the size of the state space in a goal programming model increases with the time horizon considered, and will explode when several departments of realistic sizes are considered. The drawback of simulation models is that they are 
not easily applied to other hospitals. The advantage of considering infinite server queues is that straightforward formulas for the analysis exist in the literature.

\subsubsection{Obstetrics ward}

There are several OR models that have been applied to OBS wards and maternity clinics in the literature. We describe different queueing theory approaches, a simulation model, a discrete time conditional phase type model, and a discrete time Markov model.

In research conducted almost 40 years ago, McClain [352] models the bed occupancy at an OBS ward using an infinite server queue. The ward may also admit gynecology patients to achieve higher occupancy rates, but those patients are transfered to other wards if an OBS patient has no available bed upon arrival. The gynecology patients may only be admitted to the OBS ward when the bed census is lower than a certain threshold. McClain uses an infinite server queue to represent the situation where patients are placed in unstaffed beds as a temporary measure when no official beds are available upon arrival. The results are compared for multiple hospitals when including the national guidelines regarding the admittance of gynecology patients to OBS wards, and provide guidelines for the best ward sizes.

Green and Nguyen [196] calculate the probability of delay, e.g. the probability that there is no bed available upon arrival, using an $M / M / c$ queue. Key to this model is that arriving patients who find all beds occupied wait at the clinic until a bed becomes available. During their waiting time, patients are not treated, as their 'service' commences as soon they are placed in a bed. Inputs are the average LoS found in hospital data and different arrival rates. The authors compare the probability of delay for different occupancy targets and arrival rates.

For a maternity clinic consisting of different wards, including a neonatal ward and ICU, Cochran and Bharti [111] use the Queueing Network Analyzer (c.f. [549, section 2.4.4]) to model the bed occupancy. The authors evaluate all possible bed arrangements among the wards for the peak arrival rate of the clinic. The best arrangements are then evaluated in a system with an inhomogeneous arrival rate in a discrete event simulation. Cochran and Bharti report that the hospital has implemented some of their recommendations, but instead of reassigning beds the hospital chose to add 15 beds to the ward with the highest bed shortage according to the models.

Pehlivan et al. [389] model different types of wards in a network of multiple maternity clinics with independent $M / M / c / c$ queues. The general Erlang loss formulas for the blocking probability are then fed into a mixed integer linear program to determine strategic bed assignment policies. Each year the clinic may reassign, open and close beds at the wards and clinics, and each decision entails certain costs. The authors incorporate long term planning, since it is undesirable a ward closes beds and fires nursing staff, while the next year these beds are reopened and staff is recalled. The objective of the optimization program is to minimize the costs over the decision horizon. One of their conclusions is that 
efficiency could be gained if resources are transfered among units that experience opposite demographic changes, e.g., increase or decrease in the number of women giving birth.

In an attempt to improve the occupancy rate of an obstetric clinic, Griffin et al. [200] investigate different scenarios by means of discrete event simulation. Inflow and LoS of the model are based on hospital data; patients in the model follow one of the predefined care pathways through the clinic. The authors conclude that the care pathway based approach reflects reality better than a transition probability based approach when they compare the results of both approaches to hospital data. One of the investigated scenarios includes 'swing rooms', which are rooms that can be used by multiple wards of the clinic, but not at the same time. The clinic implemented the swing rooms, which proved useful for balancing utilization throughout the clinic during bed census peaks.

Isken et al. [244] develop a discrete time Markov model to mimic a maternity clinic consisting of four wards. Patients can flow among units, with the routes patients take depending on their type. The authors define eleven patient types and six arrival streams, e.g., natural birth or cesarean, and the LoS has an empirical discrete distribution. All input is derived from hospital data. Since the model assumes infinite capacity, the authors derive the mean and variance of the bed occupancy at the units in case no patients would be deferred to other clinics. These can be used to approximate the bed census by fitting a normal distribution with the same mean and variance. The normal approximation is included in an integer linear programming optimization model to optimize the scheduled arrivals at the clinic. Several of the assumptions are validated by means of a discrete event simulation. One of the conclusions is that scheduling some patients on Saturdays smooths the bed census significantly. Isken et al. report that their model has supported multiple clinics in the United States.

The work of Harper et al. [222] focuses more on predicting the LoS of women arriving at a maternity clinic. The authors define a phase-type distributed LoS for two labor types: spontaneous and scheduled. For both types a decision tree based on patient characteristics, e.g., age and weight, further specifies the LoS parameters. The prediction of the LoS is then included in a simple continuous time Markov model to calculate bed occupancy for the labor ward of the clinic, using a homogeneous arrival rate. The model uses the LoS distribution and transition probabilities that women experience in each phase of labor. The steady state of the model reflects the bed census at different phases, which require different wards at the clinic.

In the literature on OBS wards we found two attempts at increasing bed occupancy, by either admitting non-OBS patients or by using 'swing rooms'. Interestingly, Harper et al. [222] conclude that the hospital data they obtained does not show a specific time dependent arrival distribution, while others [111, 200, 244] do model time dependent arrival rates. Arguably, scheduled arrivals likely occur only during office hours, which implies a time dependent arrival rate. Queueing models are more difficult to use in a time dependent system, since 
the simple formulas for waiting and blocking probability do not hold in a time dependent system. The drawback of using simulation models is that most models are case specific, applicable only to the clinic they were designed for. However, the advantage of a graphical simulation is that practitioners can easily see the implications of different interventions, which often implies that results of the research are more easily implemented into practice. An advantage of the discrete time Markov models is that these models have the potential to mimic reality better than queueing models, and are still more general than simulation models. However, a drawback could be a rapidly increasing state space for average sized clinics consisting of multiple wards. Isken et al. [244] propose an approximation of the bed census by a Normal distribution, and from their simulation results this seems a reasonable assumption.

\subsubsection{Weekday Ward}

Although most Dutch hospitals have a WDW and the optimization potential is significant, we were able to find only two references. This may be explained by the lack of capacity issues in these type of wards. Since all patients are elective, they can be scheduled at a time that beds are available, and patients that cannot be admitted will be accommodated on the general ward. Still, we feel that WDWs have a large logistical potential; large efficiency gains may be achieved if the number of beds is adequate and patient scheduling is optimized. Due to the lack of OR literature on WDWs, we describe two models for optimizing the patient scheduling that are relevant to the present discussion.

For a 'Monday to Friday' rheumatology clinic, Conforti et al. [120] optimize the admission to the clinic of patients from a waiting list. An introductory meeting determines a patient's medical priority, resource requirement and LoS. LoS is maximally five days. Conforti et al. develop an integer linear program, in which they decide for each resource the patient requires, e.g., beds and diagnostic tests, at which time slot it should be scheduled, if any. Each patient is assigned a weight according to his medical priority and time spend on the waiting list, while the objective is to maximize the weighted number of admissions. The authors conclude that the number of available beds is the bottleneck, and the optimized schedule can accommodate twice the number of patients compared to the schedule which was composed manually.

Braaksma et al. [64] consider an online appointment scheduling version of the WDW patient scheduling problem: a patient's request arrives and should be assigned to a date and time immediately, without knowing future patient arrivals. The authors develop an approximate dynamic programming model to obtain the optimal scheduling policy. This technique is often invoked when dynamic programming models suffer from 'the curse of dimensionality', and involves aggregating the state space and approximating the value function. 
Table 2.3 Literature on general wards categorized by applied models.

\begin{tabular}{ll}
\hline OR model & References \\
\hline Algorithms & {$[44,167,232]$} \\
Markov processes & {$[11,194,261,287,413,450,468,471,488,489,498]$} \\
Mathematical programming & {$[8,37,44,167,308]$} \\
Queueing theory & {$[36,37,44,69,180,182,192-194,196,202,224,308$,} \\
& $497,549]$ \\
Regression & {$[285]$} \\
Simulation & {$[11,25,153,157,173,194,207,223,224,232,261,277$,} \\
& $285,286,290,292,491,497,498,547,548]$ \\
Stochastic models & {$[282,333,493,498]$} \\
Time series & {$[292,334]$} \\
\hline
\end{tabular}

\subsubsection{General ward}

This section discusses models which are not applied to a specific type of ward. In the literature included in this section, general concepts are analyzed that are applicable to many types of wards, or the studies take multiple departments into account. Due to this generalization, most literature discussed in this section focuses on strategic or tactical planning by evaluating capacity dimensioning decisions or predicting demand.

The models for analyzing general concepts of bed census cover a wide range of OR techniques and are applied on different levels. The techniques used in the literature included in this subsection are given in Table 2.3. We will highlight these models and their conclusions below by discussing a selection of the papers of Table 2.3.

Shonick and Jackson [450] develop a queueing model to determine the bed demand at a community level, focusing on high occupancy rates, while keeping refusal rates of emergency patients low and waiting lists short. The bed census is modeled using an infinite server queue incorporating two classes (elective and emergency) of arrival streams. This model elaborates on earlier research applying the infinite server queue by adding threshold parameter $B$ that blocks elective admissions if the occupancy rate is higher than or equal to $B$, in order to balance the elective and emergency arrival streams. This model provides policy makers useful insights in the relation between bed census, length of the waiting list and emergency refusals. Bekker and de Bruin [36] use a queueing model that incorporates predictable fluctuations in the average number of arrivals. This time-dependent queue, an $M(t) / H / c / c$ model, is evaluated by using approximations based on the infinite server queue. It is shown that daily fluctuations have limited impact on the bed census, whereas weekly patterns do have a significant impact on both the bed census and the number of refused admissions. Finally, the authors present a method to determine the required number of beds across the week. Gorunescu et al. [192] use the $M / P H / c$ queue to determine the optimal bed census for a hospital, in which the LoS is phase-type distributed. De Bruin et al. [69] employ the Erlang loss queue $(M / G / c / c)$ to relate the blocking probability to the occupancy. Additionally, Zonderland and Boucherie [549] present 
a broad introduction of various applications of queueing networks in healthcare.

Several papers use a discrete Markovian approach to predict the short term bed census. These predictions are mainly based on the current bed census at day $t$, the expected elective and emergency admissions, and the expected discharges at day $t+j$. In these models the LoS is often empirically distributed. Utley et al. [488] approximate the census distribution resulting from their Markov model by a Normal distribution, and show that this relatively easy approximation performs satisfactory when applied to hospital wards. Markov models are also applied to obtain the distribution of the number of patients in each phase of a care pathway, for geriatric [194, 471] or stroke patients [498], in order to determine the required resources in each phase of the pathway.

Simulation is used by Dumas [153] to analyze bed allocation and usage policies for all beds in a hospital based on hospitalizations days per specialty, average daily bed census at a certain time, bed occupancy over a time period, patient misplacements and annual misplaced patient-days. Vasilakis and El-Darzi [497] invoked simulation to analyze the 'winter bed crisis', a yearly bed shortage during mid winter. The results show that discharge delays during midwinter were the main reason for the bed crises. Vanberkel and Blake [491] analyze waiting times for surgical procedures by means of simulation. Landa et al. [290] employ simulation to analyze different what-if scenarios to balance emergency and elective admissions for the available bed capacity. Keepers and Harrison [261] focus on the simulation of overflows between wards (patients are transferred to another ward because the designated ward is fully occupied), and find that the occupancy of wards is a good predictor for the frequency of overflows.

Time series models are also used to predict bed census demand. Lapierre et al. [292] predict hourly bed census demand per week with time series models. The results are used to reallocate beds between different ward types such as medical, surgical or obstetric. A different but related approach involves the use of mixed exponential equations to obtain the probability distribution of patients being in different phases of their care pathways. In [333, 498] the model is applied to mimic bed census, allocating emergency admissions on both a regional and hospital level. Results show that this type of model mimics the bed occupancy accurately. Mackay and Lee [334] study the accuracy of these mixed exponential equations based on a case study, and evaluate the effect of adding more parameters. Kortbeek et al. [282] relate the blueprint schedule of the OT, in which each subspecialty gets a fraction of the available OT time, to the hourly bed census distribution at the postoperative wards.

A nonlinear mixed integer mathematical programming model is used by Akcali et al. [8] to (re)allocate the number of available beds among different hospital services over a finite planning horizon. The decisions are based on patients' waiting time before admission and budget limits. A similar technique is employed by Van Essen et al. [167], where integer programming assists in clustering the clinical departments and assigning these clustered departments to available wards. These assignments are such that capacity is sufficient to guarantee a maximum 
blocking probability.

Concluding, the choice for a certain modeling technique depends on the desired output. Queueing theory is suitable for determining the capacity or census distribution of a single ward, preferably with mostly unscheduled patient admissions, when a maximum blocking probability or target occupancy must be achieved. Markov and time series models are accurate for determining the census distribution or certain percentiles, but might be tedious to analyze as the state space may become large. Simulation models can be developed as detailed or high-leveled as desired, but are generally suitable for obtaining average performance measures. Mathematical programming can be used to optimize the reallocation of beds to wards.

\subsection{Illustrations of OR model use}

In the previous section we reviewed several OR models applied to different types of wards. In this section we provide several detailed examples of OR models applied to an ICU, OBS, AMU and WDW. All examples are based on hospital data, and illustrate the effectiveness of OR models for certain ward types.

\subsubsection{ICU case study}

In this case study we model the bed census of an ICU of a medium-sized Dutch teaching hospital (700 beds in total). The performance measures of interest are the bed occupancy and the probability that the bed census exceeds 40 beds (the current ICU capacity). Queueing models are therefore appropriate to apply to this case study. Hospital data shows that the number of arriving patients per day is Poisson distributed, which was expected as most patients at an ICU are urgent.

Since patients at an ICU require intensive care, deferring patients or letting them wait for a bed is not a viable solution. We therefore model the ICU with the $M / G / \infty$ queue, an infinite capacity queue, so we model the system if all patients would be accepted at the ICU.

Let $\lambda$ denote the arrival rate per day, $1 / \mu$ the average LoS in days, and $\rho=\lambda / \mu$ the load of the system. In an infinite server model the number of patients at the ward at any point in time has a Poisson distribution with parameter $\rho$, cf. [519]. Therefore, the probability that $n$ beds are occupied is given by:

$$
\pi_{n}=\frac{\rho^{n}}{n !} e^{-\rho}
$$

These probabilities are independent of the shape of the LoS distribution of patients, which is convenient for modeling an ICU as the LoS distribution at ICUs typically has a high variance. In this case study, the variance of the LoS is larger than the average LoS squared.

For the ICU of this case study we find from the data that the average daily arrival rate is 2.18 patients per day, and the average LoS is 14.41 days. Therefore, 
$\rho=31.4$. Comparing the bed census from hospital data and the queueing model, see Figure 2.3, we conclude that the model underestimates the probability of an 'average census' (around 32 beds) and overestimates the probability of most other census values. From the hospital data the probability that the bed census

Figure 2.3 Bed census distribution for the ICU case study.

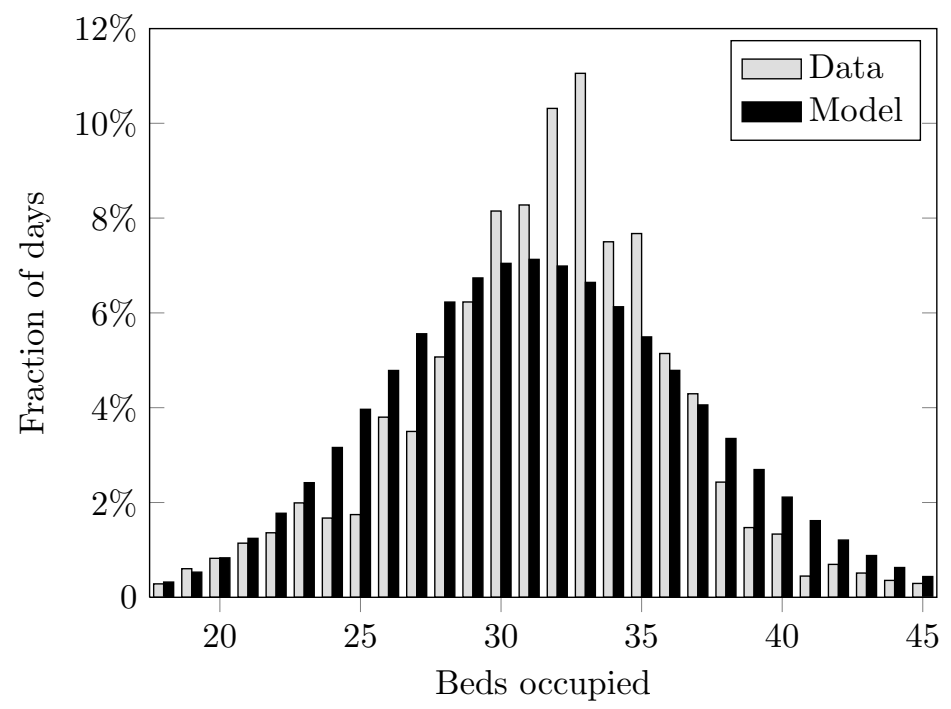

exceeds 40 equals $4.1 \%$, while from the model this is $5.6 \%$.

Note that in the hospital data, the bed census should not exceed the actual capacity, as this represents the realized occupancy. The fact that the census does exceed the capacity at some points in time, might be due to registration errors, for example when a nurse fills out all discharges at the end of the shift instead of the actual time of discharge. Additionally, the actual demand for beds is hard to obtain, since intensivists typically transfer a relatively healthy patient to another ward in case of bed shortages. This complicates the validity of the model for situations close to full capacity.

With the results of this model, hospital management can gain insight about the current performance of the ICU regarding the occupancy and the probability that the bed census exceeds capacity. Also, the effect of management decisions may be analyzed with this model, for instance the effects of bed expansion and downscaling, or an increasing average LoS through a different patient mix.

\subsubsection{OBS case study}

In this case study we model the bed census of an OBS with 24 beds. The performance measures of interest are the bed occupancy and the probability that arriving patients have to be deferred because all beds are occupied. A queueing model is therefore an appropriate choice. Hospital data shows that the number 
of arriving patients per day is Poisson distributed, which was expected as most patients at an OBS are unscheduled. The hospital data shows that the arrival rate is homogeneous over the hours, except for 8:00; at that time most of the elective patients at the ward are admitted. For the ease of modeling, we assume the arrival rate to be constant throughout the day and week. The performance measures of interest are best obtained by using a queue with finite capacity: an $M / G / c / c$ queue, also known as the Erlang loss queue.

Let $\lambda$ denote the arrival rate per day, $1 / \mu$ the average LoS in days, and $\rho=\lambda / \mu$ the average load. In the loss queue the probability density of the number of patients at the ward is given by:

$$
\pi_{n}=\frac{\rho^{n} / n !}{\sum_{i=0}^{c} \rho^{i} / i !} .
$$

These probabilities are independent of the LoS distribution of patients, which is in this case convenient as the LoS distribution at this OBS has a high variance.

For this case study we find from the data that the average daily arrival rate is 9.64 patients, and the average $\operatorname{LoS}$ is 1.14 days. Therefore, $\rho=10.96$. Comparing the bed census from hospital data and the queueing model, see Figure 2.4, we conclude that the model predicts the occupancy quite accurately. The expected number of occupied beds is 10.9 according to the model, and 11.0 according to the hospital data. From the model we obtain that the probability the ward is

Figure 2.4 Bed census distribution for the OBS case study.

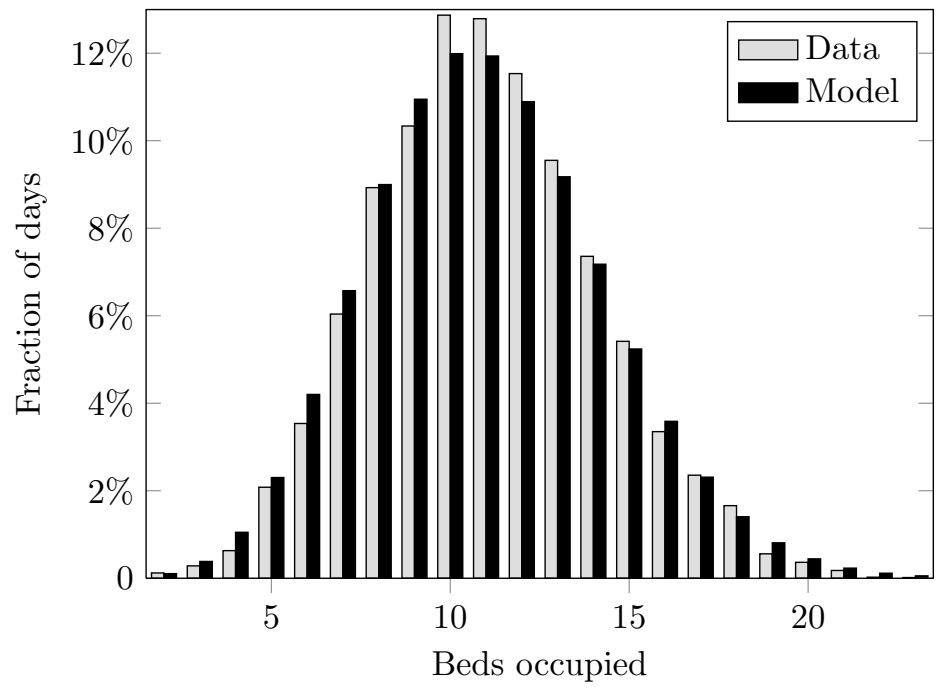

fully occupied equals $0.025 \%$. As the hospital does not register the number of deferred patients, we cannot verify this result.

The probability of a full ward is useful management information, since then hospital management can determine if the available capacity is still sufficient. 
Also this model can be used to analyze the effects on blocking probability and occupancy by changing the capacity of the ward. This is in fact easy to do, as there is a simple recursion between the Erlang loss probability for wards differing by one bed (i.e, server).

\subsubsection{AMU case study}

In this case study we consider a medium-sized Dutch teaching hospital (700 beds) that experiences difficulties with allocating urgent medical patients to inpatient beds, especially outside office hours. Typically, medical patients experience a longer ED LoS than surgical patients, partly due to a more complex diagnostic trajectory which involves observation of the patient and waiting until test results are available. As a result, EDs may become congested with these observation patients. Therefore, hospital management is considering opening an AMU to support the ED and medical departments. The purpose of the AMU would be faster admittance of ED patients that require observation or short hospitalization.

In preparation of the analysis, the doctors of the hospital have provided a list of diagnoses that can be admitted to the AMU. With this list the number of patients that would be admitted to the AMU if it were opened, can be estimated. Upon AMU discharge, patients either leave the hospital, or are admitted to an appropriate inpatient ward. The doctors agreed that discharges from the AMU would only occur during extended office hours (8:00-20:00).

The performance measures of interest are the bed occupancy and blocking probability. Queueing models are therefore appropriate to apply to this case study. As all patients are urgent and arrival rates at an ED are strongly timedependent, we model the AMU by means of an Erlang queueing model with time-dependent arrival and service rates: an $M(t) / M(t) / c / c$ queue. In a nonstationary loss queue, the limiting distribution for the number of patients in the system is time-dependent and can only be approximated. Several approximation methods exists, for example the modified offered load (MOL) algorithm [347].

The MOL algorithm approximates the load of the $M(t) / M(t) / s / s$ queue by truncating the state space of an equivalent system with infinite number of servers. Therefore the probability of having $n$ beds occupied at time $t$ is given by:

$$
\pi_{n}(t) \approx \frac{\rho(t)^{n} / n !}{\sum_{i=0}^{s} \rho(t)^{i} / i !},
$$

with $\pi_{n}(t)$ the limiting probability of $n$ patients in the system at time $t$, and $\rho(t)$ the time-dependent equivalent of $\rho=\lambda / \mu$ satisfying

$$
\frac{d}{d t} \rho(t)=\lambda(t)-\mu(t) \rho(t)
$$

Here $\lambda(t)$ is the time-dependent arrival rate, and $\mu(t)$ is the time dependent departure rate of the AMU. The MOL approximation provides good results when the system load is moderate. In systems with high load the blocking probability is underestimated. 
Figure 2.5 Arrival and discharge distribution per hour for urgent medical patients.

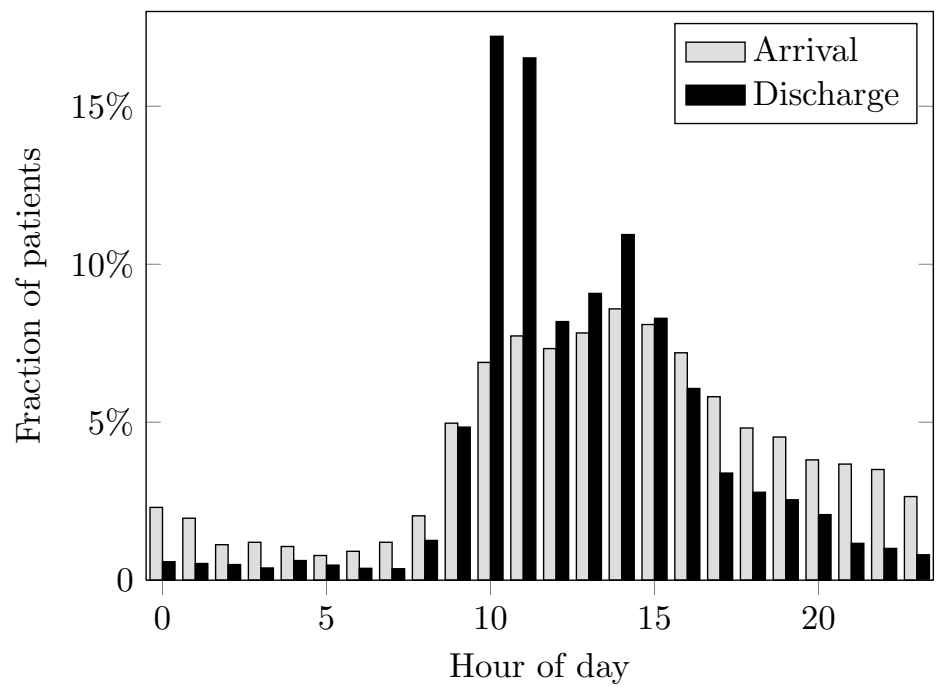

We obtain the time-dependent limiting probabilities of the number of occupied beds invoking the MOL algorithm, and use these probabilities to obtain the expected bed occupancy and blocking probability. We investigate two scenarios: admitting new patients 24 hours per day, or only during night time. Input for the model is the time-dependent arrival and service rate found in hospital data, depicted in Figure 2.5. The arrival rates are adjusted to reflect the investigated scenarios. Doctors defined the patient types eligible for the AMU, and data showed that this concerned $34 \%$ of the urgent medical patients. For the hospital

Table 2.4 Results MOL algorithm, $\bar{O}_{b c}(h)$ denotes average hourly occupancy.

\begin{tabular}{ccc|ccc}
\hline \multicolumn{2}{c}{ 24h/day } & \multicolumn{3}{c|}{ admissions } & \multicolumn{3}{c}{ Only admissions during nights } \\
no. beds & $\bar{O}_{b c}(h)$ & $P^{b}$ & no. beds & $\bar{O}_{b c}(h)$ & $P^{b}$ \\
\hline 5 & $82 \%$ & $46 \%$ & 3 & $61 \%$ & $37 \%$ \\
8 & $73 \%$ & $20 \%$ & 4 & $55 \%$ & $21 \%$ \\
10 & $66 \%$ & $9 \%$ & 6 & $43 \%$ & $5 \%$ \\
12 & $58 \%$ & $3 \%$ & & & \\
\hline
\end{tabular}

that commissioned this case study, opening an AMU is not warranted, as the bed occupancy would be low while the blocking probability would be high, as seen in Table 2.4. The number of patients that can be admitted to the AMU is not enough to achieve an acceptable bed occupancy and blocking probability simultaneously. Based on these results, the managers and doctors of this hospital decided not to open an AMU, and investigated other ways to reduce the ED crowding; this case study is presented in Chapter 3. 


\subsubsection{WDW case study}

In this case study we optimize patient admissions to a WDW, aiming to minimize the number of beds necessary to accommodate all patients at the ward. The desired model output is both the minimum number of beds required, and a cyclic blueprint patient admission schedule. This blueprint schedule specifies for each day of the week how many patients of each type may be admitted to the ward, and there should be at least enough capacity to accommodate the average number of arriving patients.

The WDW of this case study primarily accommodates outpatients (91\%), who do not stay overnight, but typically sleep off their anestetics after a simple surgery. We assume that the cycle length is 110 hours, from Monday 7:30 until Friday 21:30, as the ward closes in the weekend. Note that in this hospital patients may be discharged after office hours.

We aggregate the possible diagnoses at the WDW according to their LoS, and obtain the following patient types from hospital data:

Table 2.5 Patient types at WDW case study.

\begin{tabular}{lcc}
\hline Type description & LoS (hours) & Average no. patients/week \\
\hline LoS $<0.5$ day & 5 & 72 \\
0.5 day $<$ LoS $<1$ day & 24 & 47 \\
1 day $<$ LoS $<1.5$ day & 36 & 8 \\
1.5 day $<$ LoS $<2$ days & 48 & 3 \\
\hline
\end{tabular}

We use an integer linear mathematical programming model to solve this problem, which is known as the 'cutting stock problem' [419]. We assume each bed at the WDW is available for 110 hours. We define possible 'admission patterns': a combination of patients that could be placed consecutively in one bed within the opening hours of the WDW. For this case study we manually defined these patterns (see Table 2.6) as implementing too many patterns would not be practical. Note that for patterns 4, 7, 9, and 10, more patients with LoSs of 5 hours could be added; we assumed the maximum number of admissions per bed per day is three, to avoid admitting patients outside office hours. The mathematical program determines the minimum number of beds necessary to accommodate all patients.

Next, we define sets, parameters, variables, constraints, and an objective of the mathematical program. Let $p=1, \ldots, 11$ be the set of patterns, and $t=1,2,3,4$ the set of patient types. The parameters of the model are the demand for each patient type, $D_{t}$, and the number of patients of each type in each pattern, $A_{t p}$. $D_{t}$ is defined in the last column of Table 2.5, and $A_{t p}$ in Table 2.6. Define the decision variables of the model by $x_{p}$, the number of beds with admission pattern $p$. The objective is to minimize the sum of all $x_{p}$, while the constraints reflect that at least the average number of patients can be 
Table 2.6 Possible admission patterns for the WDW case study, with per LoS type the number of patients in each pattern.

\begin{tabular}{lllllllllll}
\hline $\begin{array}{l}\text { Pattern } \rightarrow \\
\text { LoS } \downarrow\end{array}$ & 1 & 2 & 3 & 4 & 5 & 6 & 7 & 8 & 9 & 10 \\
\hline 5 & 2 & 2 & 2 & 15 & & 5 & 10 & 6 & 8 & 5 \\
24 & & 1 & 4 & & 1 & & & & 1 & 3 \\
36 & & 2 & & & 1 & 1 & & 2 & 1 & \\
48 & 2 & & & & 1 & 1 & 1 & & & \\
\hline Sum LoS & 106 & 106 & 106 & 75 & 108 & 109 & 98 & 102 & 100 & 97 \\
\hline
\end{tabular}

accommodated. The mathematical program is given by:

$$
\min \sum_{p} x_{p}
$$

subject to:

$$
\begin{aligned}
& \sum_{p} A_{t p} x_{p} \geq D_{t} \quad \text { for all } t \\
& x_{p} \text { integer for all } p .
\end{aligned}
$$

The model can be solved with many commercially available solvers, and we chose to use Microsoft Excel ${ }^{\circledR}$. The patterns that should be used at least once and the total number of required beds are given in Table 2.7. Using this schedule the

Table 2.7 Solution of the model, PPW denotes 'patients per week'.

\begin{tabular}{lccccccc}
\hline Pattern $\rightarrow$ & 2 & 3 & 5 & 9 & 10 & 11 & Total \\
PPW per LoS type $\downarrow$ & & & & & & & \\
\hline 5 & 4 & 12 & 0 & 8 & 15 & 48 & 87 \\
24 & 2 & 24 & 3 & 1 & 9 & 8 & 47 \\
36 & 4 & 0 & 3 & 1 & 0 & 0 & 8 \\
48 & 0 & 0 & 3 & 0 & 0 & 0 & 3 \\
\hline No. beds & 2 & 6 & 3 & 1 & 3 & 4 & 19 \\
\hline
\end{tabular}

department has slots for 87 patients with LoS maximally 5 hours, and precisely enough slots for the average number of the other patient types. In total 19 beds should be enough to accommodate all patients at the WDW.

The patterns are quite flexible to use in practice as the exact sequence of the patients is not specified. During admission scheduling, the WDW has to take into account that the patients should be discharged before the ward closes on Fridays.

\subsection{Implemented OR results}

There exist many papers on OR models relating to different types of wards. It appears that the bed census and/or occupancy can be modeled quite accurately. 
However, actual use of the models in practice seems scarce; only a few of the articles reviewed for this chapter report on actual implementation results, or use of the models in practice. Dantzig [133] wrote: 'the final test of a theory is its capacity to solve the problems which originated it'. In this section we report on the problems faced with while implementing research results, and the lessons learned from the implemented research included in this chapter.

The most important lesson from the literature is that all stakeholders (not necessarily only the problem owners) should be involved throughout the entire process to increase the likelihood of implementation [111, 153, 220, 221, 223, 481]. In the phase of defining the problem, the stakeholders determine the scope of the research, relevant performance measures, and the type of output desired, for example a new admission schedule or a decision support system. When data needs to be collected for the project, stakeholders are important for retrieving data, defining the in- and exclusion criteria, and the validation of the data. Throughout the modeling phase of the project, the stakeholders are involved in several iterations of presenting and discussing preliminary results. In the last research phase, stakeholders and/or hospital management have to decide on the recommendations they want to implement, before the actual implementation can begin.

Model input determines to a large extent the outcome and the acceptance of the results. On several occasions the already available hospital data appeared to be insufficient to provide all necessary input for the models, or the database was incomplete [287, 292]. Hospital data is often inconsistent or partly missing across different databases; financial data does not always match (raw) admission and discharge data. Depending on the goals of the research, different databases may be used. Even in times of increasing use of technology, we cannot trust the data to reflect reality completely. The entry of admission and discharge data, for example, is in many hospitals still a manual task, often performed when nurses have relatively low workload or at the end of a shift. Additionally, it is important to realize that all hospital data is the realized process and most hospitals do not register deferred or denied patients, so actual patient demand is often hard to obtain. Knowing the ins and outs of the healthcare process is also essential in reading the data; for example, the LoS may depend on the bed occupancy if doctors discharge the healthiest patient to free a bed for a new patient in case all beds are occupied. A careful sensitivity analysis should be performed to ensure that the best possible scenario for implementation is included in the analysis.

Even when the analysis shows that one of the investigated scenarios is clearly superior to the others, a hospital may decide to implement (slightly) different interventions than those recommended. Discussions during projects often stimulate hospital staff and management to search for further possibilities for improvements $[153,200]$. During the project, a thorough robustness analysis should be performed to ensure that modified recommendations also improve the hospital's processes, and to avoid undesired outcomes and side-effects of the interventions. If possible, the interventions the hospital chooses to implement should be evalu- 
ated using the developed models.

There are two ways of implementation of research results: some hospitals implement the model, and some implement the management decision based on the results of the model. When a hospital is using the actual model, a researcher or third party develops a decision support tool that can either be included in the hospital's current software or used separately. The tool should match its user specific settings, or be flexible enough to be adapted to them [287, 468]. Additionally, users should be trained and supported in working with the model to ensure the continuation of the model's use [221, 468].

In some projects measuring the effects of the implementations may be difficult. Hospitals may decide to implement many different interventions at the same time [200, 287], making it impossible to distinguish the precise impact of a particular intervention. The environment in which a ward operates may change, for example when two hospitals merge or the hospital districts are redefined [232]. For prospective studies it may be unethical to measure the effect of the intervention via a randomized controlled trial, for instance: if opening an AMU appears to be the best scenario for patients, a hospital cannot set up an experiment in which one group is treated in an AMU and a control group is not. Additionally, it may also be too costly or complicated to operate a process in two different ways in parallel. Another difficulty analyzing the practical effects of an implementation is assuming default behavior by stakeholders in models. Models often optimize the overall performance, while, for instance, care professionals do not act on a system optimal level, but on individual patients. So the best option for an individual patient could be suboptimal (or worse) for the system. Therefore the results of a modeling exercise should always be accompanied by their implications for practice. When measuring the interventions' practical effects, one should take the behavior of individuals into account.

In summary, the stakeholders play a significant role in the likelihood of implementation. Additionally, researchers should be thorough in their data collection, sensitivity and robustness analyses, and implementation support. Additional information on project life cycles for general healthcare applications is found in $[220]$.

\subsection{Challenges and directions further research}

In this chapter we have discussed various OR techniques applied to different types of wards. We elaborated on to what extent these models are implemented into practice. Some models can be applied to more than one ward type and are often used in literature. We will summarize these general models, and discuss implementation and opportunities for future research.

The most commonly applied OR techniques are queueing theory and simulation. The strength of queueing models is that straightforward formulas provide quick insights in the trade-off between occupancy and blocking probability, delay, or overflow. Simulation models can incorporate more details, but require more 
development time and the results are often difficult to generalize to other wards or hospitals. Using optimization models, such as dynamic or mathematical programming, to analyze and optimize hospital wards seems a promising direction for future research, as literature in this direction is relatively sparse, and the potential of the models is high.

There is not much literature on integrating OR models into practice. Also, the literature reviewed in this chapter does not provide much insight to what extent these models are actually implemented and/or still used in practice. This may be explained by the fact that implementation requires different competences and techniques than solely OR. We are convinced that this final and for practitioners most important phase of an OR project should receive more attention both during OR projects and in OR literature, and possibly in OR education. From our own experience we know it can be challenging to make the transition from model to practice, just as doing so the other way around. Based on our findings and personal experience, we composed the following (incomplete) list of factors for successful implementation:

- Stakeholders perceive a problem.

- Stakeholders are willing to and prepared for change.

- The chain of command is involved.

- Stakeholders are involved with every phase of the analysis.

- The team thoroughly executes data collection, model verification and validation.

- The team explains practical implications of model to stakeholders.

- The team takes before and after outcome samples in order to objectively compare the effects of the implemented model in practice.

Based on the number of references per type of ward, it is also clear where the opportunities lie for OR research on wards: AMUs, OBS, and WDWs. For AMUs, it would be interesting to obtain more insights in both the medical and financial efficiency, and in which hospital settings it would be beneficial to open an AMU. Research on OBS may find better ways to cope with the high variability in patient volumes. At WDWs, it would be interesting to optimize the bed usage and access time norm compliance through better admission scheduling.

We are confidently optimistic that this chapter guides both researchers and healthcare professionals through the possibilities and opportunities OR offers for wards in balancing trade-offs between the important performance measures. 



\section{3 \\ Balancing occupancy over medical wards}

\subsection{Introduction}

Several medical wards at the Jeroen Bosch Hospital (JBH) experienced inefficient bed occupancies during 2012 and the first months of 2013. At the neurology department of the JBH patients' LoSs were reduced significantly, resulting in increasing slack capacity. Both internal medicine and pulmonary medicine experienced increasing numbers of patients, resulting in crowded wards and many patients being deferred to other wards. This chapter presents an implementationoriented case study, together with the factors that made the intervention successful.

If a ward has overcapacity, it often accommodates many patients of other medical specialties. The nurses of the neurology ward had to take care of patients from other specialties, which induces risks for both the quality of care and job satisfaction of the nurses. Additionally, many doctors from other medical specialties had to visit the neurology ward, who all give their instructions to the nurses and thereby disrupt regular care processes.

In case a ward has undercapacity, finding suitable other wards for the patients consumes much time, and doctors have to make long rounds to visit all their patients at different wards. The quality of care may be at stake if patients are not treated at the medically preferred ward, or experience an additional transfer if a bed becomes available at their preferred ward.

The JBH aims to balance the occupancy of the medical wards, in order to warrant the quality of care and job satisfaction. From the previous chapter it appears there are many OR models applied to hospital wards, but the results of these modeling efforts are not often implemented in practice. In accordance with the list of factors in Section 2.7, we commissioned a steering group consisting of all stakeholders in this problem: a neurologist, an internist, a pulmonologist, a stakeholder from the admission management office, and all involved (ward) managers.

In this chapter we describe a case study of redesigning patient flows at multiple medical wards of the JBH. We present the case study in Section 3.2 and the investigated interventions in Section 3.3. The results of this research are implemented at the JBH, and in Section 3.4 we show that the intervention improved 
the processes as intended. Additionally, we present promising decision rules to support the process in practice.

\subsection{Before intervention}

The total bed capacity of the JBH is 730 beds, of which 64 beds are at the neurology ward, 32 at the pulmonary medicine ward, and 96 at the internal medicine ward. The layout of each unit is depicted below, including the medical specialty the unit is assigned to. Here, ' $23+9$ ' denotes that one of the neurology units has nine monitored beds reserved for patients who had a stroke, and 'neu+pul' denotes that neurology and pulmonary medicine already work together as overflow departments; nurses at the neurology department are trained to take care of pulmonary patients, and pulmonary patients are often accommodated at the neurology ward.

\begin{tabular}{|c|c|}
\hline 1. Neu $23+9$ & 2. Neu+pul 32 \\
\hline 3. Pul 32 & 4. Int \\
\hline 5. Int 32 & 6. Int \\
\hline
\end{tabular}

Figure 3.1 Bed census and available beds for neurology and pulmonary medicine.

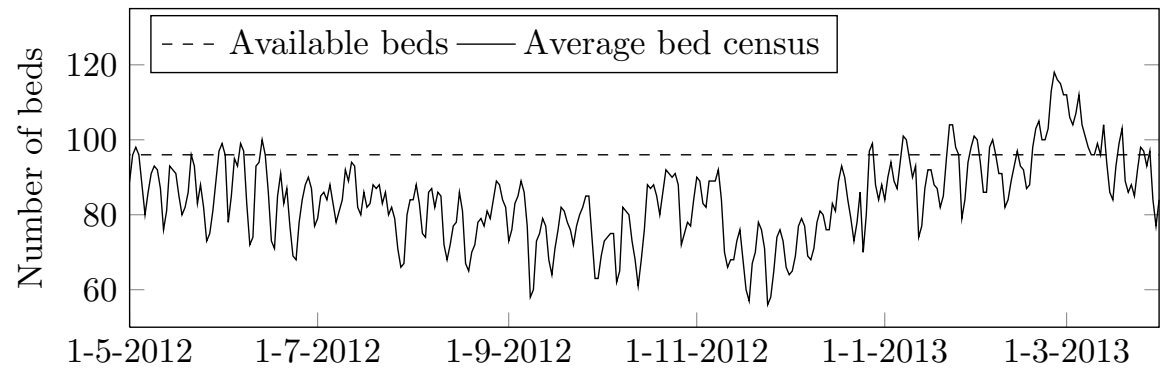

Figure 3.2 Bed census and available beds for internal medicine.

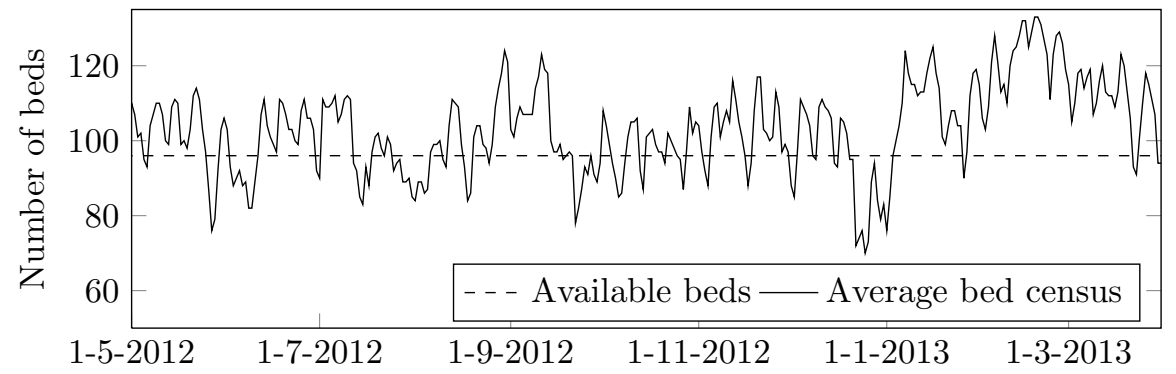

Historical data (from 1-4-2012 to 1-4-2013) confirms that neurology and pulmonary medicine had slack capacity and internal medicine experienced regular 
Figure 3.3 Bed census and available beds for all involved medical specialties.

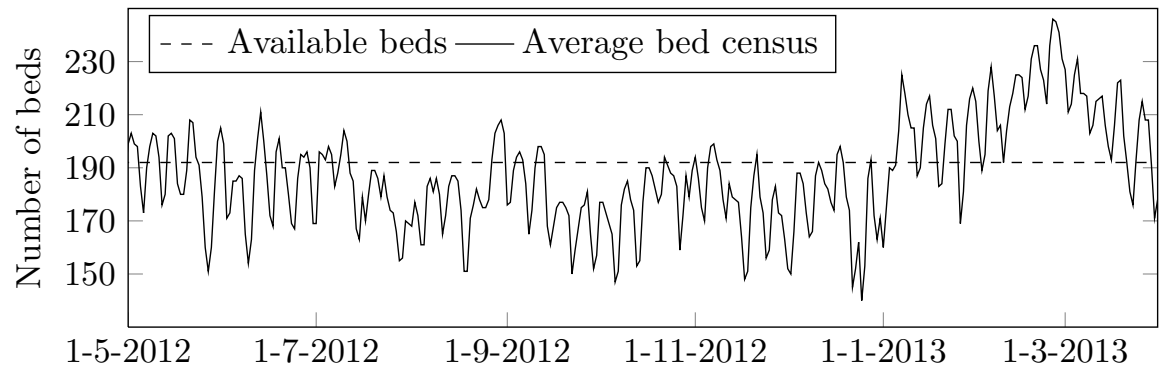

overflows, see Figures 3.1 and 3.2. In these figures the average daily bed census for each medical specialty is given, opposed to the bed census at the involved specialty wards, in order to provide insights in the actual bed usage for each specialty, including the misplaced patients. Note that the bed census for neurology and pulmonary medicine peaks in the beginning of 2013; this Dutch winter was relatively cold, resulting in extreme numbers of patients with pneumonia. If we would have pooled all beds from the involved medical specialties, most patients could have been accommodated at this aggregated ward, see Figure 3.3. Therefore, adding bed capacity to any of the wards seems unnecessary.

\subsection{Intervention}

It appears from the literature review in Section 2.4.5 that both queueing theory and mathematical programming may be used to reallocate medical specialties to the available bed units. Hospital data indicates that over $77 \%$ of the patients are urgent, implying that queueing models could give accurate estimates of the occupancy and blocking probability. As the number of possible reassignments for the involved specialties is little, queueing models are preferred over a mathematical programming approach.

In order to estimate the bed requirements for each specialty individually, we determined the minimal capacity to achieve at most $5 \%$ blocking probability using an $M / G / c / c$ queue: internal medicine requires 99 beds, pulmonary medicine 43 , and neurology 47 (including the stroke patients). The bed occupancies for these numbers of beds according to the queueing model would be $95 \%, 86 \%$, and $88 \%$, respectively. The total number of beds at the involved units is 192, and the model indicates that 189 beds are required, confirming that additional capacity is unneccessary. However, it should be noted that in reality patients are often not 'blocked and lost' if all beds at their medically preferred ward are occupied upon their arrival. If fewer patients are blocked, this implies that the $M / G / c / c$ queue underestimates the bed occupancy.

The steering group has investigated three possible interventions:

1. opening an AMU,

2. reassigning medical specialties to the units,

3. organizing an overflow ward for internal medicine. 
Each of these interventions will be discussed in the remainder of this section.

Assigning urgent patients from internal medicine to appropriate beds has often appeared to be challenging at the JBH. A large proportion of urgent internal medicine patients who arrive at the emergency department require observation and/or wait for several diagnostic test results. An AMU for medical patients should warrant quick admission for (among others) these urgent patients, thereby alleviating congestion at the emergency department. The effects of this intervention were analyzed using an $M(t) / M(t) / c / c$ queue. Input for this model was an estimation of the hourly arrival rate, for which internists and pulmonologists indicated which possible diagnoses were medically eligible to be admitted to an AMU. Several scenarios were investigated, such as admitting patients all day or only outside office hours, and an allowed total LoS of 24 or 72 hours (after which patients are either admitted to a regular ward or discharged). For the description and a selection of results of this model, see Section 2.5.3. The analysis indicated that the size of an AMU would not be large enough to achieve both acceptable occupancy rates and blocking probabilities, as the aim of the JBH is $80-90 \%$ occupancy while the blocking probability is below $5 \%$. Moreover, opening an AMU would imply an additional transfer for part of the patients, and may negatively affect elective patient admissions (see Section 2.4.2).

For the second intervention, reassigning medical specialties to units, all possible reassignments for the specialties were investigated. The newly formed ward assignments should assign 99 beds to internal medicine, 43 to pulmonary medicine, and the remaining 50 to neurology. The reserved beds for stroke patients stay assigned to neurology in all scenarios. The most promising investigated scenarios were:

\begin{tabular}{|l|l|}
\hline 1. Int $(+9$ neu $)$ & 2. Neu \\
\hline 3. Pul & 4. $\operatorname{Int}(23) \operatorname{neu}(9)$ \\
\hline 5. Int & 6. $\operatorname{Int}(21) \operatorname{pul}(11)$ \\
\hline
\end{tabular}

\begin{tabular}{|l|l|}
\hline 1. $\operatorname{Int}(14) \operatorname{neu}(9+9)$ & 2. Neu \\
\hline 3. Pul & 4. Int \\
\hline 5. Int & 6. $\operatorname{Int}(21) \operatorname{pul}(11)$ \\
\hline
\end{tabular}

Here, 'Int(21) pul(11)' denotes that internal medicine is assigned 21 beds at the unit and pulmonary medicine 11. Each of the scenarios required serious rebuilding of units or medical specialties being split up among multiple wards. Therefore, the steering group decided not to reassign the medical specialties to the wards.

The third intervention, an overflow ward for internal medicine patients, is based on the concept of 'pooling resources': internal medicine would share beds at the neurology ward with it's current users. The organized overflow ward is also beneficial for neurology, as the neurology ward nurses prefer that overflow patients accommodated at their ward have comparable diagnoses. Not all internal medicine patients are eligible to be accommodated at the neurology ward, as some patients require specialized care. Therefore, internists determined a list of diagnoses that were eligible to accommodate at the neurology ward. Together with emergency department data on occurring diagnoses, we obtained that maximally $34 \%$ of the urgent internal medicine patients were eligible overflow patients, 
who would occupy on average eight beds if they were all accommodated at the neurology ward. Note that internal medicine would be assigned three beds at the neurology ward to achieve the norm on the blocking probability, if the beds would not be pooled. Both numbers of beds were acceptable for the steering group. After investigating training requirements for nurses and setting rules for doctors' rounds, from September 2013 all eligible, internal medicine, overflow patients were primarily accommodated at the neurology ward.

\subsection{Results}

In January 2014 we measured the effects of the intervention on two performance measures: the average hourly bed occupancy and the number of unnecessary patient transfers. Additionally, we interviewed nurses and doctors from both neurology and internal medicine.

Table 3.1 Occupancy before and after the implementation (Jan. '12 - Jan. '14).

\begin{tabular}{lcc}
\hline Averages & Before & After \\
\hline Occupancy neurology ward & 45.25 & 44.87 \\
of which neurology & 37.98 & 36.82 \\
of which pulmonary medicine & 5.80 & 5.14 \\
of which internal medicine & 0.77 & $2.55(\mathrm{p}=0.00)$ \\
of which other specialties & 0.71 & $0.36(\mathrm{p}=0.03)$ \\
Occupancy internal medicine ward & 80.77 & 80.88 \\
Occupancy pulmonary medicine ward & 24.85 & 24.53 \\
\hline
\end{tabular}

Table 3.2 Unnecessary transfers per patient before and after the implementation (Jan. '12 - Jan. '14).

\begin{tabular}{lccc}
\hline Medical specialty & Before & After & Difference \\
\hline Internal medicine & 0.62 & 0.25 & $-60 \%$ \\
Other specialties & 0.10 & 0.05 & $-50 \%$ \\
\hline
\end{tabular}

Although the difference in the occupancy is little, internal medicine patients occupied significantly more beds at the neurology ward, while the number of patients from other medical specialties decreased, see Table 3.1. The number of unnecessary patient transfers is not registered at the JBH, but was estimated using data. We included all patient transfers if the patient was first accommodated at the neurology ward and: (1) the patient's specialty was not changed, and (2) the new ward was not a ward with a higher level of care, such as the ICU. The intervention decreased the number of unnecessary transfers per patient significantly for both internal medicine and other overflow patients at the neurology ward, see Table 3.2 .

The intervention additionally resulted in a reduction of the time internists required for their rounds. The neurology nurses experienced a reduction of the fluctuations in the workload and were confident to deliver high quality of care. A 
downside of the implementation appeared to be a higher workload at the internal medicine ward, as many of the 'easier' patients were admitted to the neurology ward.

\subsection{Additional improvements}

Although the intervention proved to work in practice, the staff responsible for admitting patients requested a decision rule to determine to which ward each patient should be assigned. To this end, we conducted a discrete event simulation, in which we used historical admission and discharge data for internal medicine, pulmonary medicine and neurology patients.

In the simulation, each arriving internal medicine patient is eligible to overflow with a probability of $34 \%$, as the JBH admission data did not contain diagnoses. We investigate different decision rules, all of 'threshold type':

1. admit all eligible overflow patients to the neurology ward,

2. only overflow if $x$ or fewer beds are available at the internal medicine ward,

3. only overflow if $y$ beds or more are available at the neurology ward,

4. only overflow if both $x$ or fewer beds are available at the internal medicine ward and $y$ or more beds are available at the neurology ward.

If an overflow patient cannot be admitted to the neurology ward, he is admitted to the internal medicine ward if possible, and otherwise considered 'blocked and lost'; in reality, for this assumption to hold, they would be accommodated at a different ward elsewhere in the hospital and would not be transfered to their preferred ward even if a bed becomes available. If the other arriving patients cannot be accommodated at their preferred wards, these patients are considered blocked and lost. The investigated performance measure is the blocking probability.

From the simulation results it appears that decision rule 1 would result in relatively high blocking probabilities for neurology and pulmonary medicine patients. The results of decision rule 3 would be similar if the threshold value is not set above 15 beds. Both rules 2 and 4 result in similar outcomes and appear to be promising for implementation. Based on the simulation results, such as depicted in Figure 3.4, the steering group decided to implement decision rule 4 with $x=3$ and $y=2$.

\subsection{Conclusion}

In 2012 and the first months of 2013 the bed occupancies of internal medicine, pulmonary medicine and neurology were unbalanced. To improve this balance, internal medicine patients with specific diagnoses were allowed to be accommodated at the neurology ward. This intervention improved the balance regarding bed occupancy and reduced unnecessary patient transfers. A simple decision rule was determined to support the patient admission process.

In this case study medical specialists proposed interventions, and the effects of these interventions were prospectively investigated. All medical specialists 
Figure 3.4 Simulation results of decision rule 4 , with $y=2$.

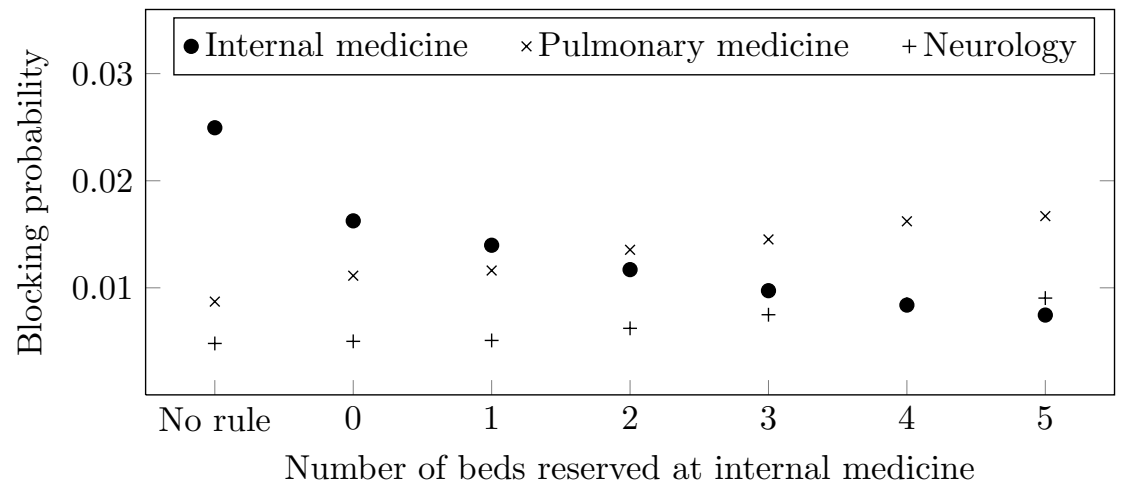

focused on improving processes for patients, averting discussions on competing interests of the individual specialists. Since the effects of all possible interventions were quantified in advance, the steering group was able to choose the most promising intervention to implement. This intervention was supported by all involved staff, and proved to be effective in reality.

The occupancy and blocking probability of the overflow system may be modeled using an overflow queueing model, such as the one presented in [552]. The steering group decided that the improved accuracy of the estimations would not change the chosen intervention, which implied that the overflow model was out of the scope of this case study. However, it would be interesting to analyze in future research. Additionally, for this chapter we analyzed an isolated AMU using queueing theory. It would be interesting to extend this model such that it additionally encompasses the emergency department and involved wards. In such a setting the overflow of patients to and from different departments could be investigated, making the AMU serve more as a buffer department. For the JBH case specifically, in future research it would be interesting to investigate the effectiveness and efficiency of an AMU on a larger scale, with more hospital wards involved. 



\section{Part II}

\section{Online appointment scheduling}

Chapter 4

A. Braaksma, N.M. van de Vrugt, and R.J. Boucherie. Online appointment scheduling: a taxonomy and review. Submitted.

\section{Chapter 5}

N.M. van de Vrugt, N. Litvak, and R.J. Boucherie. Blocking probabilities in Erlang loss queues with advance reservation. Stochastic Models, 30(2):187-196, 2014. 



\section{4}

The state of the art in online appointment scheduling

\subsection{Introduction}

Appointment scheduling is the act of assigning a customer's request for service to a future day and time, and to a resource. There are many industries in which appointments are scheduled: jobs are scheduled on machines, patients are scheduled in the calendars of healthcare professionals, data transmission requests are scheduled on a route of links, et cetera.

The quality of appointment scheduling is assessed according to several performance metrics. From a customer's point of view, accessibility and completion times are important: with what probability is a customer admitted into service at a given facility, how long does the customer have to wait for service, and when is the service completed? For the service provider, utilization and profitability are important metrics: how should the service provider schedule appointments efficiently, and which customers should be selected for service in order to maximize revenues? The objectives of customers and providers may conflict; for example, inserting idle time in a schedule improves customer waiting time, but often decreases resource utilization.

Two different modes of appointment scheduling can be distinguished: offline and online. In offline appointment scheduling (also referred to as static or allocation scheduling), the scheduler collects all service requests for a given service period before the appointments are scheduled. Thus, at the moment of scheduling all demand is known, and resource calendars are empty. Comprehensive reviews on offline appointment scheduling are provided in [76, 83, 212, 362, 400]; offline scheduling is outside the scope of this chapter. In online appointment scheduling (also referred to as dynamic or advance scheduling), customers receive a prompt response to their appointment request. Thus, appointments are scheduled in partly filled resource calendars, when future demand for the same service period is still unknown. Online appointment scheduling has attracted considerable research attention recently, and is a rapidly expanding field. However, this field of literature has not been reviewed yet. In this chapter, we provide an overview of the state of the art in online appointment scheduling.

The contribution of this chapter is threefold. First, we provide an overview of 
a rapidly growing field of literature. Second, we identify open research problems. And third, by not restricting our search to any one application area, we provide an overview of the literature spanning various fields. In doing so, we aim to stimulate mutual interchange of research results in the field of online appointment scheduling.

This chapter is organized as follows. In Section 4.2, we demarcate the scope of our literature review and present our taxonomy. We elaborate on our findings in Section 4.3, followed by a discussion in Section 4.5.

\subsection{Scope and taxonomy}

In this section, we formalize the setting used for the study of online appointment scheduling, followed by the taxonomy used in this chapter, and a clarification of terminology frequently used in each application area.

\subsubsection{Scope}

We consider a facility consisting of one or multiple resource types. Each resource type has one or more servers. Customers' appointment requests arrive dynamically over time, indicating when the customer would like to receive service from which resource types and for how long. Customers may request service as soon as possible, or specify one (several) time window(s) in the future in which they want to be served. When a new request arrives, the scheduler must promptly decide if the customer is allowed access to the system. If a request is accepted, the scheduler must give the customer an irrevocable appointment date and time, at which access to the facility is warranted. The actual time the customer is served might differ slightly from the scheduled starting time of the appointment due to, for example, other appointments running late. The scheduler may either notify customers of their appointment times promptly upon their request (online scheduling), or at specific periodic time points (near-online scheduling).

Two fields of literature that are closely related to the described setting are outside the scope of this review. First, we exclude the field of revenue management, which focuses on predicting customer behavior, and optimizing order acceptance and resource pricing for several application areas. Typical output of revenue management methods is a set of pricing levels and/or booking limits, prescribing a maximum number of each customer type to accept. Many reviews on revenue management models can be found in the literature, listed here by application area: airplanes [31, 384], car rental [530], dynamic pricing [48, 52], e-commerce [62], hotels [245], industry [363], recreational systems [447], service industries [205], trains [19], transportation [353], and general appointment systems [103]. Second, we exclude the literature on online bin packing, which studies how to assign a set of items of different sizes to a minimum number of bins with finite capacity. This field of literature has recently been surveyed in [115].

In this chapter, we focus on recent developments in operations research on online appointment scheduling. We performed initial searches on the databases 
Web of Knowledge and Scopus using the search string "(online OR advance*) AND appointment AND schedul*", including manuscripts complying with the scope described above. Starting from this initial set of manuscripts, we exhaustively searched the literature by reviewing both the references cited in, and citing the included manuscripts. Because of the publication policy differences in various scientific fields, we included both papers published in peer-reviewed journals and selected conference publications (mostly available in IEEE Xplore). To ensure we provide an overview of the state of the art, we additionally included recent high-quality unpublished manuscripts that are available online, or have been obtained through personal communications. The literature search was updated up to 30 November 2014.

\subsubsection{Taxonomy}

We categorize the literature according to a taxonomy with two axes, comparable to the one in [242]; the vertical axis represents the scheduling horizon and the horizontal axis the number of appointments and resource types, as depicted in Figure 4.1. We elaborate on these axes below.

The vertical axis of our taxonomy represents the scheduling horizon on which the appointment planning is done, and consists of capacity allocation ('cap'), near-online appointment scheduling ('near'), and online appointment scheduling ('onl').

The online scheduling horizon considers systems in which arriving customers promptly receive a response to their appointment request. Hence scheduling is done for one customer at a time, and this customer has to be scheduled before the next customer becomes known. Scheduling therefore takes place in partly filled resource calendars.

At the near-online level, there is some delay between the arrival of a customer's request and assigning an appointment to this customer. Appointment scheduling is done periodically at specific points in time, for all demand that

Figure 4.1 Taxonomy for online appointment scheduling and overview of the chapter.

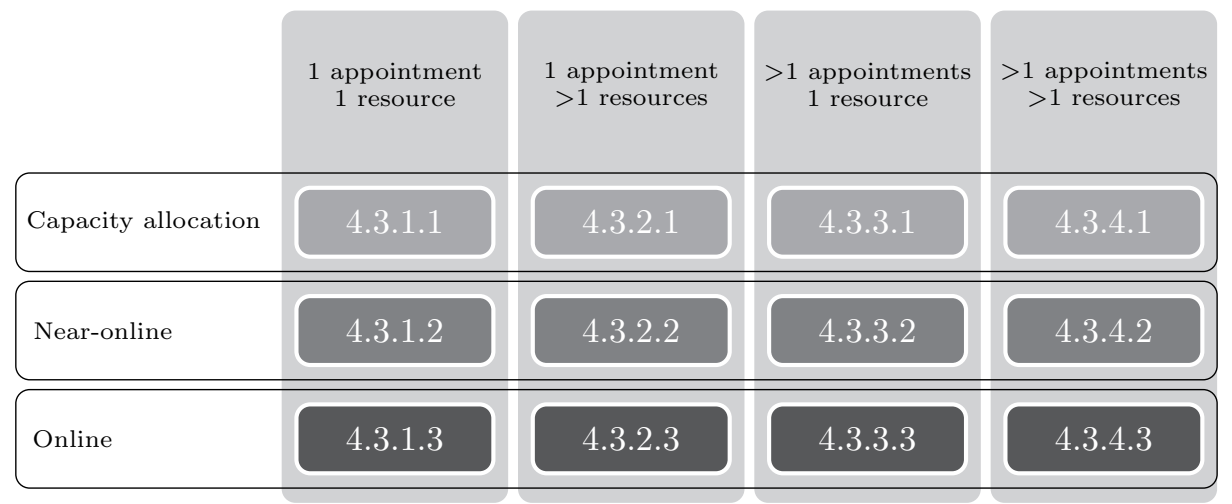


has arrived until that time. For example, scheduling can be done at the end of each day, based on all customer requests that have arrived during that day. Hence customers may experience some waiting time before receiving a response to their appointment request, scheduling can be performed for a number of customers simultaneously, and, similar to the online case, it takes place in partly filled resource calendars.

At the capacity allocation level, the available resource capacity is subdivided over time and over different customer types. Decisions include, for example, when which servers will be available to serve customers, and which slots are reserved for which customer types. At this level customers are not scheduled explicitly, but managers determine boundaries that significantly influence the possibilities for (near-)online appointment scheduling, which typically are blueprint schedules or booking limits. A blueprint schedule reserves time slots for specific customer types, while a booking limit prescribes a maximum number of customers (per type) that is allowed access to the facility.

The horizontal axis of the taxonomy represents the number of appointments and resource types that each customer requests. We categorize the literature into four categories:

1. One appointment on one resource type ('1-1'). Each customer requests one appointment and the system consists of only one resource type.

2. One appointment on multiple resource types ('1-m'). Each customer requests one appointment, the system consists of multiple resource types, and there is at least one customer type that requests multiple resource types simultaneously.

3. Multiple appointments on one resource type (' $m-1$ '). Each customer requests one or more appointments, the system consists of only one resource type, and there is at least one customer type that requests multiple appointments.

4. Multiple appointments on multiple resource types (' $m$ - $m$ '). Each customer requests one or more appointments, the system consists of multiple resource types, and there is at least one customer type that requests multiple appointments, and at least one customer type that requests multiple resource types.

When a system consists of multiple resource types but each customer requests service from one resource type only, known upon the customer's arrival, we also categorize such literature in categories 1 and 3 , as such a system can be seen as a collection of 'one resource type' systems, where each customer is directed to one of the subsystems according to the customer's required resource type. Also, note that category 4 contains two types of studies: first, studies in which customers have appointments with one resource at a time, but require different types of resources for different appointments, and second, studies in which customers have multiple appointments, at least one of which at multiple resource types simultaneously. 
In Section 4.3, we discuss the findings of our literature search based on this taxonomy, using the structure depicted in Figure 4.1. To increase readability, in the remainder of this chapter we use the following notation for referring to the categories in this taxonomy: 'cap', 'near', and 'onl' for the horizontal axis, and ' 1 ' or ' $\mathrm{m}$ ' for one or multiple appointments or resource types. For example, near-1-m refers to near-online scheduling of one appointment on multiple resource types.

\subsubsection{Terminology}

In different application areas, different terminology is used for similar notions. Moreover, several application-specific definitions can make reading a paper from a different application area a difficult task. Therefore, we clarify the terminology used in this chapter.

We use the term (appointment) slot to refer to the smallest time window in which one customer can be scheduled. Blocking probability refers to the fraction of denied customers. The time between the request and the scheduled starting time of the appointment is access time, and customers may specify an access time window, indicating the period within which they prefer having their (first) appointment scheduled. The time between the customer's arrival at the facility and the actual starting time of the appointment is waiting time, and we define the sojourn time as waiting time plus service/processing time. The planning horizon is the number of future days, weeks, or months the scheduler considers as options for scheduling a customer's appointment. Customers not attending their scheduled appointments without prior notification are called no-shows. Customers without appointments can be emergency customers, who have to be served immediately after arrival, or walk-in customers, who may wait for a while. The terms admission control and order acceptance refer to policies for deciding which customers are or are not allowed to access the system. In lead time quotation, the scheduler mentions an estimate of the customer's sojourn time, and the customer then decides whether to accept that or to leave the system.

Because the field of machine scheduling makes a significant contribution to the relevant literature, we introduce some definitions from this field. Makespan minimization refers to minimizing the completion time of the job that finishes last. The quality of an online scheduling algorithm is usually judged based on its competitive ratio: an online algorithm is said to be $\rho$-competitive if for any instance of the problem the objective value of the schedule generated by the algorithm is at most $\rho$ times larger than the optimal offline objective value.

Another application area often mentioned in this chapter is healthcare. We use the abbreviation $O T$ for operating room or theater. A master surgical schedule $(M S S)$ is a blueprint schedule in which the operating time and rooms are assigned to medical (sub)specialties. Patients admitted to hospital wards and staying overnight are called inpatients, whereas outpatients do not stay overnight and only visit the hospital for consultations, examinations, or treatment, taking place in outpatient clinics. Patients who are non-urgent and scheduled in advance are elective, and scheduling elective inpatients is called admission planning. Ra- 
diotherapy and chemotherapy are treatments generally used to cure cancer or to provide patients with relief from its symptoms, using respectively radiation or drugs to destroy cancer cells.

In communication applications, packets of data request to be transfered from a source to a destination via a network. In this application, a grid is a collection of computer resources on multiple locations that can be used in parallel. In Optical Burst Switching networks, sets of packets with similar properties, called bursts, are sent after reserving an optical channel in advance.

\subsection{Results}

In this section, we present the results of our literature review. In addition to categorizing the papers according to our taxonomy, we subdivide papers based on study objective. We distinguish four categories of objectives, each clarified below.

Accessibility. Accessibility relates to objectives regarding the access time, blocking probability and server assignment. Typical trade-offs relating to this objective are: should the current customer with a broad access time window be scheduled in the near future to prevent server idleness, or should capacity in the near future be kept free in anticipation of arriving customers with tight access time windows? Would refusing the current customer result in a lower global (all customers together) blocking probability? When customerto-server assignment preferences or restrictions play a role: to which server should the current customer be assigned to maximally enable (optimal) server assignment of future customers?

Profitability. Authors considering profitability aim to maximize the revenue gained from serving customers. Typical trade-offs are: how much capacity should be reserved for high-profit customers and how much may be consumed by low-profit customers? Should a current low-profit customer be accepted to ensure the associated revenue, or should capacity be kept free to possibly gain higher revenue? In problems where routing also plays a role: which customers should be assigned to which route to minimize transportation costs?

Utilization. In this category, we group several objectives relating to the utilization of the system's resources: maximizing utilization; maximizing throughput (e.g., maximizing the number of customers served per unit of time); balancing the utilization (over several servers or over time); balancing server idle time, server overtime, and customer waiting time; achieving a target utilization; and providing insight in utilization (e.g., studies revealing the effects of certain admission policies on resource utilization). We mention a few of the numerous trade-offs relating to these objectives: should customers' appointments be scheduled consecutively during a service session, in order to minimize server idle time and overtime, or should there be idle time in between 
Table 4.1 The number of manuscripts found per category.

\begin{tabular}{llcccc}
\hline & & $1-1$ & $1-\mathrm{m}$ & $\mathrm{m}-1$ & $\mathrm{~m}-\mathrm{m}$ \\
\hline Capacity & Accessibility & 26 & 1 & 5 & 8 \\
allocation & Profitability & 8 & 1 & - & 2 \\
& Utilization & 58 & 1 & 2 & 25 \\
& Completion times & - & - & - & 1 \\
\hline \multirow{2}{*}{ Near-online } & Accessibility & 17 & 4 & 9 & 5 \\
& Profitability & 3 & 1 & 1 & 3 \\
& Utilization & 6 & - & 2 & 2 \\
& Completion times & 8 & 3 & - & 3 \\
\hline \multirow{5}{*}{ Online } & Accessibility & 30 & 12 & 2 & 17 \\
& Profitability & 8 & 2 & 1 & - \\
& Utilization & 42 & 13 & 1 & 3 \\
& Completion times & 33 & 5 & 2 & 19 \\
\hline
\end{tabular}

to minimize customer waiting times? Which customers should be accepted or rejected in order to achieve a target utilization?

Completion times. The category completion times contains the following objectives: minimizing the completion times of individual jobs (also, minimizing sojourn times); minimizing the overall system completion time (makespan minimization); and finishing jobs as close to their deadlines as possible (penalty costs could be incurred both for early and late completions). Typical tradeoffs in this category are: to which time slot and machine should the current job be assigned, in order to minimize the makespan? Should the current job be scheduled to finish exactly at its deadline, or would scheduling it at a different time yield better possibilities for future jobs?

Our literature search led to a set of 1712 manuscripts. We based the inclusion decision on the following steps: judgment about whether the studied model is inside our scope (see Section 4.2.1), exclusion of manuscripts included in one of the reviews we include, and avoidance of similar contributions by the same authors. As a result, we included 341 manuscripts in this chapter. Table 4.1 gives an overview of the number of included manuscripts per category; we reflect on this table in Section 4.5. Note that the sum of the entries in Table 4.1 is larger than 341 , as some manuscripts appear in multiple categories.

Next to categorizing each manuscript based on our taxonomy and its objective, we have classified each manuscript according to the following items:

- Application area: communication, healthcare, production, service, transportation, or general/unspecified.

- Operations research methodologies: an overview of the operations research methodologies considered, including a short description of each, is provided in Appendix 4.6. See Section 2.3.1 for more mathematical details on the models. 
- Number of servers per resource type: one or multiple?

- Blocking: may the scheduler deny requests?

- Time: do the authors consider a discrete or a continuous time model?

- Optimization: are there optimization components, or is it an evaluation study?

- Future: does the scheduler anticipate future appointment requests when scheduling the current appointment(s)?

Per category in our taxonomy, we now discuss our findings, subdivided by the four categories of objectives. We discuss major application areas, scheduling decisions, trade-offs included in these decisions, and operations research methodologies used to support decision making. Since our set of manuscripts is large and each manuscript has been classified according to a considerable number of items, we restrict ourselves to key features. The detailed classification of all manuscripts can be found in the supplementary Excel ${ }^{\circledR}$ document, available at www.utwente.nl/choir/bibliography.xlsx.

\subsubsection{One appointment on one resource type}

\subsubsection{Capacity allocation}

Accessibility Access time objectives for cap-1-1 are prevalent in healthcare applications: outpatient clinics [23, 126-128, 145, 159, 252, 270, 281, 298, 420, 535], diagnostic examinations [183-186, 301, 386, 428, 504], and OT scheduling [233, $342,541]$. A few articles study a problem in a general context [164, 330, 438]. We distinguish three types of research questions within this category: how many appointment slots to offer and when to offer these slots [126, 127, 159, 438, 535]; how many appointment slots to reserve for each customer type [23, 128, 145, 164, 183$186,233,252,301,330,342,386,428,504,541]$; and which appointment slots to reserve for each customer type $[270,281,298,420]$. In the third question, the timing is important, because these studies include, next to customers making appointments well in advance, customers who should be served at short notice: within one or two days after their appointment request [270, 420], on the day of their request [298], or shortly after they have walked into the service facility [281].

Next to keeping customers' access times within preset targets, additional objectives studied are: minimizing overtime [127, 145, 301, 438, 535]; minimizing customer waiting times [127, 535]; balancing customer waiting time, server idle time and overtime [270, 298, 420]; maximizing system utilization [183186, 301, 386]; minimizing the blocking probability for emergency customers [23]; and minimizing the probability that a walk-in customer cannot be served within a certain time frame [281].

Several authors [128, 145, 233, 252, 301, 330, 342, 386, 428, 541] generate a static allocation of appointment slots to customer types, whereas other studies [23, 164, 183-186, 504] allow for flexibility, for example by making the allocation dependent on the current system state [23, 164], releasing reservations under 
certain circumstances [183-186, 504], or increasing the number of appointment slots offered in busy periods by temporarily extending opening hours [504].

Methods: algorithms [23, 128, 145, 164, 184, 185, 233, 281, 330, 342, 504], dynamic programming [23, 183, 185], mathematical programming [184, 233, 252, 342 , 386, 541], queueing theory [126-128, 145, 159, 186, 252, 281, 330, 535], simulation $[145,159,164,184,186,270,298,301,330,386,420,428,438,504$, 541], stochastic models [164, 281, 438]

Profitability The vast majority of research in this category is in the field of revenue management; see Section 4.2 for a brief description of this field and references to the extensive literature. Other types of applications can be found in healthcare and transportation. In healthcare, problems studied are how to (re)allocate OT time to different surgical specialties [140, 211, 461, 473], and how many elective patients of different medical specialties to admit to nursing wards on each day in a given planning horizon [512], both with the objective of maximizing hospital revenues. In transportation, dynamic vehicle routing problems are considered (see [399] for a recent review), in which delivery time slots have to be assigned to customers before actual demand is known. The decision is which time slot or set of time slots to assign to each customer or to a set of customers in a geographical zone, and how to construct vehicle routes, with the objective of minimizing total transportation costs and maximizing revenues gained from accepting requests $[6,110,460]$.

Methods: algorithms [6, 110, 140, 460, 461], forecasting [110, 461], mathematical programming $[6,140,460,473,512]$, networks and graphs $[6,110,460]$, review [211, 399], simulation [110, 461, 473]

Utilization The majority of papers studying utilization objectives for cap-11, design a blueprint appointment schedule for an outpatient clinic, a diagnostic facility, an OT, or a general appointment system. (See Table 4.2 for an overview of the references relating to this paragraph.) Five different decisions are studied: the number of appointment slots to offer per session, the starting times of patients' appointments during a consultation session, the number of patients to schedule in each slot, which type of appointment system (open access or overbooking) is best for which parameter settings, and the optimal length of the planning horizon. Most articles consider a (weighted) combination of the objectives mentioned in Table 4.2. While all studies consider patients who request an appointment well in advance, additional patient types considered are: same-day or open access patients, who request an appointment on that same day; walk-in patients, who arrive at a facility without an appointment; inpatients, who are admitted to the hospital and can be scheduled on very short notice; and emergency patients, who require treatment as soon as possible after their arrival, without an appointment.

Some studies consider no-shows, and the probability of patients not showing up for their appointment can depend on their type [10] or access time [74, 171, 298, 407, 408, 414, 539]. The authors assume that patients with shorter access 
Table 4.2 Characteristics of articles designing blueprint appointment schedules.

\begin{tabular}{|c|c|}
\hline \multicolumn{2}{|l|}{ Application areas } \\
\hline Outpatient clinic & $\begin{array}{l}{[10,15,27,28,74,82,84,99,127,129,256,269-272,281,289,} \\
298,318,325,390,407-409,414,420,458,465,470,535-537, \\
539]\end{array}$ \\
\hline Diagnostic facility & {$[53,77,198,451]$} \\
\hline Operating room & {$[139]$} \\
\hline General & {$[171,262,320,505,526]$} \\
\hline \multicolumn{2}{|l|}{ Decisions } \\
\hline No. appointments & {$[74,127,407,408,414,535]$} \\
\hline Start times appointments & $\begin{array}{l}{[10,15,77,84,99,129,139,262,271,272,320,325,409,458,} \\
470,505,526]\end{array}$ \\
\hline No. patients per slot & $\begin{array}{l}{[27,28,53,82,198,256,269,270,281,289,318,390,420,451,} \\
465,536,537,539]\end{array}$ \\
\hline Open access or overbooking & {$[298]$} \\
\hline Length planning horizon & {$[171]$} \\
\hline \multicolumn{2}{|l|}{ Objectives } \\
\hline Min. access times & {$[10,127,535]$} \\
\hline Min. waiting times & $\begin{array}{l}{[15,82,84,99,127,129,139,198,256,262,269-272,289,298,} \\
318,325,390,409,420,451,465,470,505,526,535-537,539]\end{array}$ \\
\hline Min. idle time & $\begin{array}{l}{[15,82,84,99,129,139,256,262,269-272,318,390,409,420,} \\
470,505,526,536,537]\end{array}$ \\
\hline Min. overtime & $\begin{array}{l}{[15,77,82,84,99,127,129,139,256,270-272,289,298,318,} \\
325,390,409,420,470,505,535-537,539]\end{array}$ \\
\hline Max. no. treated & $\begin{array}{l}{[10,27,28,53,74,77,171,198,289,320,325,407,408,414,} \\
451,539]\end{array}$ \\
\hline Min. no. rejections & {$[53,198,281,298,320,451]$} \\
\hline Max. revenue & {$[10]$} \\
\hline Balance utilization & {$[458]$} \\
\hline \multicolumn{2}{|l|}{ Additional patients } \\
\hline Same-day patients & {$[27,28,74,99,298,390,407,408,414]$} \\
\hline Walk-in patients & {$[82,84,281,390,465]$} \\
\hline Inpatients & {$[53,198,451]$} \\
\hline Emergency patients & {$[53,198,272,325,451]$} \\
\hline Including no-shows & $\begin{array}{l}{[10,74,129,171,256,289,298,320,325,407,408,414,470,537,} \\
539]\end{array}$ \\
\hline
\end{tabular}

times are more likely to show up for their appointments. For elaborate overviews of outpatient appointment scheduling, see [83, 212, 213, 385].

In addition to studies designing blueprint appointment schedules, we have found three other types of studies in this category of our taxonomy. First, $[50,104,235,264,464]$ decide how much OT time to assign to different surgical specialties, with the objective of minimizing both over- and underutilization (see [76, 217] for recent reviews on OT planning and scheduling). Second, [179] studies admission planning in outpatient procedure centers, which solely provide routine elective treatment (see [43] for an overview of appointment scheduling in such centers). In [179], the authors decide how many patients of each type to admit on each day of the week, with the objective of achieving contractual obligations on the number of patients treated yearly. Third, [275, 493] develop 
models that provide insight in bed utilization on nursing wards resulting from a given admission policy [275] or master surgical schedule [493].

Methods: algorithms $[27,50,77,82,99,104,139,171,198,235,256,264$, $271,272,281,289,325,390,409,414,451,505,526,537,539]$, dynamic programming $[74,171,198,275,414]$, forecasting [84, 526], mathematical programming $[27,50,77,99,104,139,171,179,235,264,272,325,409,458]$, queueing theory [53, 127, 262, 275, 281, 320, 535], review [43, 76, 83, 212, 213, 217, 385], simulation $[10,15,28,77,82,84,139,171,269-272,275,298,318,325,390,409$, 420, 458, 465, 505, 526, 537], stochastic models [15, 129, 171, 179, 256, 281, 289, $318,407,408,464,470,493,505,536]$

\subsubsection{Near-online scheduling}

Accessibility We identify three different types of research questions considering accessibility objectives for near-1-1. The first question is to which future day each customer's appointment request should be assigned, studied in a general context [165], and in various healthcare settings: operating rooms [187, 417], diagnostic facilities [387, 482], chemotherapy [189], outpatient clinics [322], and inpatient admissions [40, 351, 435]. Key issues are that multiple patient types have different access time targets [165, 189, 387] or different planning horizons [187, 351, 482], and all patients must be scheduled such that the sum of the costs for violating the access time targets and 'diverting' patients (e.g., treating patients in overtime or referring patients to another healthcare provider) is minimized. [322] includes cancellation and no-show probabilities, which increase with patients' access times. By means of the appointment scheduling game [432], healthcare professionals and students can experience the challenges of this problem.

The second question studied also appears in healthcare, and is called the patient admission scheduling problem: to which room (e.g., considering medical specialty or the patient's gender) should each elective patient be assigned? [40, 435] decide on both the admission date and the room assignment, while [86, 87, 494] assume admission dates to be predetermined, although [87] allows for slight deviations from these predetermined dates.

The third type of articles are from the field of communication. In the video on demand problem $[30,218]$ customers request a particular video from a central video server, which must be sent to the customer via a data channel as soon as possible [30] or within a specified preferred access time [218]. The decision is which customers to accept or to reject, and in which time slots to serve accepted customers. For a network for videoconferencing, [219] minimizes the blocking probability using admission control.

Methods: algorithms [30, 86, 87, 189, 218, 219, 322, 417, 435], dynamic programming [165, 187, 189, 322, 387, 482], mathematical programming [40, 87, 351, 417, 435, 494], scheduling [30], simulation [189, 218, 219, 322, 432, 435], stochastic models [435] 
Profitability The studies in this category aim to maximize total revenues by accepting or rejecting customers in manufacturing systems [225, 394] or in the workplace training industry [9]. In [9] the scheduler additionally assigns each accepted request to a trainer, as there are multiple types of trainings and each trainer can perform a subset of these.

Methods: algorithms [9], dynamic programming [9, 225, 394], mathematical programming [225], simulation [394]

Utilization We distinguish three different utilization-related objectives among the studies on near-1-1. The first is studied in healthcare: to provide insight in the effects of different admission policies on utilization, taking the uncertain arrivals of emergency patients into account [275]. The second objective is to balance utilization, either over time $[170,206]$ or over the different servers in the system [215]. This is important for minimizing the delay in travel times for highway users [170], maintaining relatively constant rates of production (production smoothing) [206], and achieving high performance in large-scale multi-processor systems [215]. The third objective is to maximize utilization; either by admission control in an optical burst switching network [257], or by scheduling applications to hosts (computing nodes) such that the total number of hosts used is minimized [280].

Methods: agent-based models [170], algorithms [170, 206, 215, 257], dynamic programming [275], mathematical programming [280], queueing theory [275], networks and graphs [257], simulation [170, 206, 257, 275]

Completion times The majority of papers optimizing completion time objectives in near-1-1 consider makespan minimization in a parallel machine scheduling problem with a reordering buffer $[100,101,143,146,359]$. Upon arrival, jobs either have to be irrevocably assigned to one of the machines and a starting time slot, or can temporarily be stored in the finite-capacity buffer. The machines are either identical [143, 359], uniformly related (the machines work at different speeds) $[143,146]$, or hierarchical (each job specifies a subset of machines on which it can be processed) [100, 101]. In [359] rejection of jobs is allowed at penalty costs that differ per job, and the objective is to minimize the sum of the makespan and the penalty costs of the rejected jobs.

The other articles in this category deal with completion times relating to individual jobs. Problems studied are: order acceptance with hard deadlines [338], lead time quotation in make-to-order systems [21], and making a reservation at the second queue in a service system consisting of two queues, such as to minimize customers' sojourn time [529].

Methods: algorithms [100, 101, 143, 146, 338, 359], dynamic programming [338, 529], queueing theory [21, 529], scheduling [100, 101, 143, 146, 359], simulation $[21,529]$ 


\subsubsection{Online scheduling}

Accessibility The objective of complying with access time norms for onl-1-1 is most studied in outpatient clinics. The key issue is at what time to schedule less urgent patients, such that their access time is within the norm, the resources are utilized efficiently, and enough slots are available for more urgent patients. Some clinics reserve capacity for each patient type by using a blueprint schedule, and a scheduler then has to decide to which slot each patient should be assigned. To enhance solution quality, certain studies develop policies allowing patients to take slots that are reserved for other types of patients [184, 210, 503]. In other clinics, patients either take the first available slot on the same day [197], or are assigned to an appointment day [545] or day and time [429] in the future. [197, 429] incorporate no-shows, and [429] investigates overbooking to increase efficiency. [197, 503] additionally take patients' preferences into account. See $[83,212,213,385]$ for elaborate reviews on appointment scheduling in outpatient clinics.

In amusement parks [283] and call centers [18], customers can choose between waiting in the queue or making an appointment for a future time (sometimes referred to as 'virtual queueing'). Key issues here are to determine the optimal policy for serving customers with and without appointment, and to estimate the waiting time based on the current queue length including the future customers who have previously decided to make an appointment.

For a packet transferring application, [135] determines the optimal number of reserved positions for delay-sensitive packets, with respect to the access time of both delay-sensitive and delay-tolerant packets.

Instead of considering access times, several papers analyze and/or minimize the blocking probability, in different applications: in outpatient clinics that defer patients who cannot be treated within opening hours [310, 551], home delivery for online retailers where customers request a delivery time window [156], dynamic vehicle routing with time windows [42], video on demand systems [30], communication networks $[79,92,239,258,260,418,466,506,546]$, and general reservation systems [142, 154, 305, 510]. Of these papers, some consider both the blocking probability and resource utilization [79, 92, 210, 239, 260, 310, 418, 466, 546].

Methods: algorithms [30, 79, 142, 156, 239, 283, 305, 310, 429, 466, 503], dynamic programming [184, 545], mathematical programming [239, 429], queueing theory [18, 135, 197, 260, 283, 418, 506, 510, 551], review [42, 83, 92, 212, 213], scheduling [30, 142, 154], simulation [79, 156, 197, 210, 239, 258, 260, 283, 305, $310,418,466,503,506,510,546]$, stochastic models $[258,546]$

Profitability There are many revenue management applications considering profitability, see Section 4.2 for several literature reviews of this field. Optimizing the scheduler's profit is also important in various other applications: the pickup and/or delivery of full truckload orders, in case the trucks are managed by local agents who can place bids for orders [357]; assigning each grocery delivery (of different volumes) to a delivery vehicle and time-window [72]; and accepting or 
rejecting drivers who pay for a guaranteed crossing of an intersection [499] or access to a downtown area [543]. In the first two applications, the key issue is taking the location of future requests into account in placing bids [357] or finding the shortest delivery route [72]. In the latter two applications, customers can choose between free service with possibly high waiting time or paid guaranteed service, which is also studied for a general appointment system, in [452]. See [399] for a review on vehicle routing with time windows. For general appointment systems, [230,303] optimize order acceptance while taking the expectation of future requests into account, if necessary using overtime or outsourcing [230].

Methods: agent-based models [357, 499], algorithms [72, 230, 499], dynamic programming [230, 357], game theory [357, 452], mathematical programming [303], networks and graphs [72, 357], neural networks [543], queueing theory [303], review [399], simulation [230, 543]

Utilization The majority of the papers optimizing utilization-related objectives for onl-1-1 consider outpatient clinics. In these clinics it is important to balance patients' waiting time, doctors' idle time and clinic overtime [89, 125, 136, 166, 312, 321, 327, 365, 385, 429, 484, 514, 525, 537, 539]. Other papers assist outpatient clinics by providing insight in the resource utilization $[28,88,210,310]$, or maximizing the resource utilization [169]. Some studies consider additional patient types (see Section 4.3.1.1-Utilization): walk-in patients $[321,514,525]$, or same-day patients $[28,166]$. Schedulers have to decide on: which reserved appointment slot to assign a specific patient to [210, 537, 539], the appointment time on a specific day [89, 136, 166, 169, 310, 312, 365, 484, 525], the best among the appointment times the patient prefers [514], the appointment day (taking the first available slot on that day) [125], or both appointment day and time [429]. Assuming that patients take the first available slot on the same day and the clinic treats a mixture of walk-in patients and patients with appointments, additional questions are: the optimal panel size (maximum number of patients) of the doctor [321,327], how many same-day patients to accept on one day [385], and to which doctor each patient should be assigned $[28,210,514]$. For the latter question, clinics prefer to schedule patients with their regular care provider, even when the patient could get an earlier appointment with an other provider and that other provider risks being idle. Patients with appointments can have a certain probability of noshow $[88,89,125,312,321,327,365,429,484,537]$, in which case clinics might consider overbooking. See $[83,212,213,385]$ for recent reviews on appointment scheduling in outpatient clinics.

A relatively new application is alleviating road traffic congestion by means of advance reservation. The goal of accepting or rejecting vehicles on highways [316], intersections [148, 499], or in downtown areas [543], is often twofold: the manager wants to maximize both the revenue and the throughput of the road. An expected positive side-effect of requiring payed reservations is the increase in the number of people carpooling, which is better for the environment. Key issues in this type of 
problems are the different routes drivers take (e.g., left-turning or right-turning traffic at an intersection), and that different vehicle types (e.g., trucks, cars) have different characteristics in terms of size and speed.

For communication networks [32, 79, 92, 239, 418, 466, 542, 546], and general reservation systems $[113,114,260,362]$, the objective relevant for this review is to provide insight in utilization. In $[51,112,507]$ the utilization is maximized by means of an admission policy for general reservation systems. For a loading bay advance reservation system, [355] studies the robustness of the schedule.

Methods: agent-based models [499], algorithms [28, 51, 79, 89, 148, 169, 239, $310,316,365,429,466,499,507,514,525,537,539,542]$, decision analysis [112], dynamic programming $[28,312,507,514]$, mathematical programming $[89,166$, $429,484]$, queueing theory [32, 88, 112-114, 125, 136, 260, 321, 327, 418], review [83, 92, 212, 213, 362, 385], scheduling [51], simulation [28, 79, 148, 166, $210,239,260,310,316,355,418,466,484,507,537,539,542,543,546]$, stochastic models $[32,89,239,365,525,546]$

Completion times Optimizing completion times for onl-1-1 is prevalent in the machine scheduling literature. Online scheduling methods optimize the assignment of jobs to machines with respect to the makespan. The machines can either be identical $[149,172,259,297,306,328,329,444,459,531,540]$, uniformly related (each machine operates at a different speed) [16, 22, 161-163, 251, 323], or hierarchical (jobs specify a subset of machines on which they can be processed) [101, 253, 259, 297, 323, 328, 329, 444, 540], and few papers study both identical and uniformly related machines [209, 319]. A job can have a deadline (see the reviews $[12,296,404,443,469]$ ), or be rejected against certain costs $[163,209]$. In some settings preemption is allowed; a scheduler is allowed to divide the job in several parts and schedule the parts (non-overlapping) at different machines $[162,163]$. Some studies consider regular working hours and overtime [459, 531], or machines that can be down for planned maintenance [172, 319].

The scheduler may have some information about the future when making the scheduling decision (called 'semi-online' scheduling): all job sizes lie in a certain (small) interval [149, 540]; jobs arrive in decreasing order of processing times [162, 306] or rejection penalties [163]; or certain characteristics of the problem are known, such as the sum of all processing times [16, 101, 297, 323], or of a subset of the jobs [101, 328]; the size of the largest job [297, 323]; the optimal objective value [297, 323]; or the processing time of the next job that will arrive [329]. Next to minimizing makespan, other objectives used are: minimizing makespan and rejection costs [163], or minimizing total completion/busy time [444, 459, 531].

In other applications, completion time is important for determining visit time windows for service engineers visiting customers [254, 335]; order acceptance for home deliveries in metropolitan areas [156] or order acceptance in general [454]; vehicle routing with time windows [399]; or minimizing individual completion times in scheduling computation requests for a computer resource [150].

Methods: algorithms [22, 101, 149, 150, 156, 161-163, 172, 209, 251, 253, 254, 
$259,297,306,319,323,328,329,335,444,459,531,540]$, networks and graphs [335], review [12, 296, 399, 404, 443, 454, 469], scheduling [16, 22, 101, 149, 161163, 172, 209, 251, 253, 259, 297, 306, 319, 323, 328, 329, 444, 459, 531, 540], simulation $[150,156]$

\subsubsection{One appointment on multiple resource types}

\subsubsection{Capacity allocation}

Accessibility Complying with access time norms for cap-1-m is important at an endoscopy (nonsurgical procedures to examine the digestive organs) department; patients require an appointment with, for example, a nurse, doctor, and procedure room at the same time [255]. In developing a cyclic schedule, the decision is how many slots to assign to each procedure type, and at which times.

Methods: mathematical programming [255], simulation [255]

Profitability The problem of maximizing revenue for cap-1-m is found in a computer grid [467]. Job brokers aim to select the most profitable resources for their jobs, based on prices and available times quoted by independent resource owners. [467] determines pricing levels and booking limits for the resources on the grid. The goal is to maximize the profit, by maximizing the resource utilization and adjusting the booking limits to the demand forecast.

Methods: agent-based models [467], algorithms [467], forecasting [467], simulation $[467]$

Utilization The utilization objective for cap-1-m applies to a cancer clinic: patients require a treatment chair throughout the entire appointment, and two nurses and one pharmacy technician at the beginning of the appointment [240]. The goal is determining a blueprint schedule for a clinic day that maximizes resource utilization.

Methods: mathematical programming [240], simulation [240]

\subsubsection{Near-online scheduling}

Accessibility Maximizing the (weighted) number of accepted requests is important in communication networks. For computer grids [326, 358], scientific research networks ('e-science') [412], or optimal burst switching networks [175] it can be beneficial to accumulate several requests first, before a (virtual) central scheduler decides which requests to accept. Each subtask of a request should be scheduled such that pre-specified Service Level Agreements (SLAs) are met, otherwise the entire request is rejected. For some applications, the scheduler must also assign the request to a specific route on the network the moment the request is accepted [175, 412], and requests can have different priorities or rewards $[175,358]$. 
Methods: algorithms [175, 358, 412], mathematical programming [412], networks and graphs [175], queueing theory [326], simulation [175, 326, 358, 412]

Profitability The only paper we have found considering profitability for near$1-\mathrm{m}$ is [188]. The authors optimize the admission policy for a general appointment system in which jobs can be rejected, outsourced or processed in overtime against certain costs.

Methods: dynamic programming [188], simulation [188]

Completion times Sojourn time (propagation delay) [495, 553] and system completion times $[412,553]$ are important performance measures for transferring large datasets before a given deadline. Two decisions must be made consecutively: which jobs to accept, and how many channels on which route(s) to assign to each job.

Methods: algorithms [412, 495, 553], mathematical programming [412], scheduling [412], simulation [412, 495, 553]

\subsubsection{Online scheduling}

Accessibility Many studies considering accessibility for onl-1-m are applied to computer networks. These networks consist of multiple resources that are managed by a central scheduler. Each job consists of a data packet that should be transmitted from a source to a certain destination in the network, and all links on the job's route should be available simultaneously. As there typically are multiple routes from source to destination, the scheduler has to decide which route the job will take over the network after accepting a job. A job request can consist of: a certain number of resources for a specific time interval in the future [204, 341, 418, 434, 480, 523]; a certain number of resources for a 'flexible' (larger than the duration of the job) interval [361, 478]; or a deadline before which the job must be completed [80,382]. This field of literature either analyzes the blocking probability analytically [418, 480], or develops new scheduling algorithms and evaluates them with respect to the blocking probability (and the resource utilization) $[80,341,361,434,457,478,523]$. In some papers, offline optimization is used for comparison [204, 457]. In [523], jobs are allowed to overlap, exploiting the fact that most jobs overestimate their running time. See [92] for a review on scheduling in computer networks.

Methods: algorithms [80, 204, 341, 361, 382, 434, 457, 478, 523], mathematical programming [204, 457], networks and graphs [457], queueing theory [418, 480], review [92], simulation [80, 204, 341, 361, 382, 418, 434, 457, 478, 480, 523]

Profitability Profitability is important in assigning requests for different skill sets to multi-skilled technicians, either for telecommunications installations [33] or for housing repair and maintenance [130]. Requests have different rewards, and may specify both rejection costs and costs for the total time spent in the 
system (i.e., access time plus sojourn time) [33]. In [130], the scheduler may use overtime or subcontractors.

Methods: algorithms [33, 130], dynamic programming [33], mathematical programming [33], queueing theory [33], simulation [130]

Utilization Utilization objectives for onl-1-m are important for computer network applications. Many papers on appointment scheduling in computer networks consider both the blocking probability and the resource utilization $[80,341,361,434,457,478,523]$. In this paragraph, we classify the papers that only consider the resource utilization, as papers considering both accessibility and utilization are included in the accessibility paragraph of this section. The scheduling decision is both to decide whether to accept or reject an arriving job, and which route the job will take over the network. A job request can consist of: a certain number of resources for a specific time interval [455]; a certain number of resources for a flexible interval (larger than the duration of the job) [485]; a deadline before which the job must be completed [383]; or a certain number of resources and negotiable starting time [304, 456]. See [92] for a review on scheduling in computer networks.

A different objective in appointment scheduling for computer networks is the minimization of energy consumption by the network. Each resource in the network consumes energy while it is switched on, even when it is not processing jobs [383]. Switching components off is only beneficial if the off-period is long enough, so the question is when to switch components off and at what times to schedule the jobs in the on-periods.

Methods: algorithms [80, 304, 341, 361, 383, 434, 455-457, 478, 485, 523], mathematical programming [457], networks and graphs [457], review [92], simulation [80, 304, 341, 361, 383, 434, 455-457, 478, 485, 523]

Completion times Next to the blocking probability and system utilization, individual completion times are important performance measures in computer network applications. The central [108, 553] or decentralized [108] scheduler decides whether to accept or reject an arriving job, and which route the job will take over the network. Jobs can request service in a flexible time window [553] or before a given deadline [108], and may have different priorities [553]. See [92] for a recent review on scheduling in computer networks.

Completion time objectives for onl-1-m can also be found in the machine scheduling literature, see [296, 469] for recent reviews.

Methods: algorithms [108, 553], review [92, 296, 469], simulation [108, 553]

\subsubsection{Multiple appointments on one resource type}

\subsubsection{Capacity allocation}

Accessibility Accessibility objectives for cap-m-1 are considered in healthcare applications: radiotherapy [307, 379, 477], chemotherapy [117], or outpatient 
clinics [374]. Each patient requires a series of appointments, and different patient types have different access time targets, treatment frequencies, and appointment lengths. All studies develop blueprint schedules allocating capacity on specific days and time slots to the different patient types, aiming to comply with access time targets as much as possible. Additionally, [307, 374] determine the minimum number of resources required for meeting the access time targets, while [117] balances the workload of the resources over time.

Methods: algorithms [117, 307, 374, 379, 477], mathematical programming [117, 307, 374], networks and graphs [374], queueing theory [307], simulation $[307,379]$

Utilization Optimizing utilization-related objectives is important in the 'consistent vehicle routing' problem, which exists in home care planning [375] and many other application areas [284]. In home care planning, patients require a series of appointments and should consistently be visited by the same nurse. $A$ priori routing (designing a set of routes in advance based on predicted demand) decreases nurses' travel times, increasing their utilization [375].

Methods: algorithms [375], networks and graphs [375], review [284]

\subsubsection{Near-online scheduling}

Accessibility Minimizing access times for near-m-1 is important for radiotherapy treatment scheduling. Here, each patient type has its own access time target, number of required treatments, and treatment frequency. A patient can only be scheduled if all his treatments can be scheduled. The scheduler decides each day $[70,300,397,398,433]$ or week $[118,119,121,247]$, when to schedule the new appointment requests, sometimes allowing for treatment interruptions [398], or for a small number of appointments being omitted [121]. In two studies [300, 433], forecasts of future appointment requests are taken into account in scheduling the new appointment requests.

Methods: algorithms [300, 397, 398], dynamic programming [433], mathematical programming $[70,118,119,121,247,300,433]$, simulation [70, 247]

Profitability The only study found optimizing profitability for near-1-m considers hadron therapy (a special type of radiotherapy) treatment admission and scheduling [513]. A part of the patients may be deferred to receive conventional radiotherapy, and the objective of the scheduler is to maximize the center's profit by deciding which requests to accept and on which days to schedule the appointments of each accepted patient.

Methods: mathematical programming [513]

Utilization Utilization-related objectives are important in the 'consistent vehicle routing' problem, which exists in many application areas [284], including home care planning [41]. Home care patients require a series of appointments 
with certain inter-appointment time and should consistently be visited by the same nurse. A scheduler accumulates new requests and schedules them periodically with the objective of maximizing the number of patients served per unit of time.

Methods: algorithms [41], review [284], simulation [41]

\subsubsection{Online scheduling}

Accessibility The two papers considering accessibility for onl-m-1 minimize the blocking probability in a general appointment system [24], and aim to comply with access time preferences in a radiotherapy application [299]. [24] studies the competitive ratios of randomized algorithms (in which the scheduler takes an action with a certain probability) for accepting requests. In [299], the decision is on which days to schedule the treatments, additionally aiming to minimize the use of overtime.

Methods: algorithms [24, 299], mathematical programming [299], scheduling [24]

Profitability Maximizing profit for onl-m-1 is important in scheduling orders consisting of multiple packages on a single resource type [229]. The scheduler uses demand forecasts to decide if an order is accepted, and at which day and time it is executed by which machines (including additional capacity like overtime or outsourcing), in such a way that the deadline of the order is met.

Methods: dynamic programming [229]

Utilization The only research we have found considering a utilization objective for onl-m-1, is for a chemotherapy application [117] (also mentioned in the accessibility paragraph in Section 4.3.3.1). A scheduler allocates a patient to a series of appointments in the blueprint schedule, aiming to balance the workload of the nurses.

Methods: algorithms [117]

Completion times Individual [7] and system [55] completion times are important in scheduling jobs consisting of several tasks on a communication network link. Tasks may have precedence constraints and deadlines [7], and may be scheduled in parallel. The scheduling decision is at what time each task should be assigned to which channel.

Methods: algorithms [7, 55], networks and graphs [7], simulation [7, 55]

\subsubsection{Multiple appointments on multiple resource types}

\subsubsection{Capacity allocation}

Accessibility Booking limits, or a maximum number of customers that can be accepted, are used frequently for complying with access time norms, mostly 
in healthcare applications for cap-m-m. For clinical trials, [137] optimizes how many patients can start with each type of trial each day, such that access times are within the norms and the scientific value of the trials is maximized. The time between each appointment of a trial is predetermined and cannot be violated. A similar decision problem can be found in scheduling patients for nuclear medicine [392], in which timing is also crucial; if one appointment in the series cannot be scheduled in the required time interval, the whole series has to be scheduled at a later time.

In a general hospital setting, [243] determines how many patients of each type (diagnostic group) should be scheduled on each resource in order to comply with access time norms and given targets on the number patients to treat yearly, while minimizing the total time patients spend in the system. [501] determines the required capacity to comply with access time norms at a multidisciplinary outpatient clinic. [502] considers a diagnostic facility in which the number of patient visits to the clinic is reduced by combining multiple appointments on one day [502]. This objective is also studied in OT scheduling, see [76, 217] for recent reviews.

Methods: mathematical programming [47, 137, 243, 392, 501], queueing theory [501], review [76, 217], simulation [47, 137, 392, 501], stochastic models [137]

Profitability Maximizing profit for cap-m-m is important in the container shipping industry. Customers request that a container with a certain size, weight, and electricity requirement, is shipped from an origin to a destination at or before a certain time. Based on empirical demand forecasts, central management determines limits for the number of requests that can be accepted from each customer group (defined by budget and request characteristics). Reservations are then either centrally managed [554], or by decentralized management at each port [138].

Methods: agent-based models [138], algorithms [138, 554], mathematical programming [138, 554], simulation [554]

Utilization The utilization objective for cap-m-m is predominantly found in OT scheduling. The goal is to develop an MSS in which the OT time is divided among all medical specialties, such that all specialties receive enough OT time to perform their elective surgeries. Since elective patients are canceled if there is no bed available at their post-operative ward, taking patients' bed requirements into account is essential for creating a suitable MSS. To this end, for each block in the MSS (i.e., subgroup of patients with common characteristics) the required post-operative resources and length of stay at those resources are estimated using historical data. Common objectives for optimizing the MSS are: to level the bed occupancy at the subsequent wards [38, 39, 105, 177, 266, 331, 381, 426, 430], or minimizing the difference between the realized and a target occupancy [2, $3,75,508]$, but one can also maximize the throughput while taking resource availabilities into account [29]. The resulting MSS specifies for each operating 
room on which days and times a certain medical specialty can use it. See $[76,217]$ for reviews on OT scheduling.

Similar types of optimization problems are found in making oncologists' schedules for a chemotherapy outpatient clinic [426], setting patient appointment times for outpatient clinics [228, 238, 421, 522] and a primary care practice [380], deciding how many patients for each specialism can be admitted into the hospital $[376,544]$, and reserving routes on a highway [155]. The objectives in these papers are to balance utilization over different days of the week [228, 426], balance waiting and idle time [238, 380, 522], maximize throughput [421], achieve a target utilization $[376,544]$, and maximize utilization [155].

Methods: algorithms [2, 38, 39, 78, 155, 177, 331, 380, 381, 421, 426], dynamic programming [376, 544], mathematical programming $[2,3,29,38,39,75,78$, $105,155,177,228,331,380,381,421,426,430,508]$, networks and graphs [155], queueing theory [228], review [76, 217], simulation $[2,29,75,78,105,238,266$, $331,421,522,544]$, stochastic models [177, 228]

Completion times Individual completion times are minimized for a chemotherapy outpatient clinic in which all patients request three appointments that must be scheduled on one day [214]. The authors develop a blueprint schedule which prescribes possible starting times for the series of appointments for each patient type.

Methods: constraint programming [214]

\subsubsection{Near-online scheduling}

Accessibility Accessibility objectives in near-m-m are studied in healthcare. Patients requiring radiotherapy request multiple appointments with various resources, sometimes with multiple resources simultaneously $[81,396]$. The scheduler assigns all patients to days and time slots, minimizing both the access time and sojourn time. Other healthcare applications are chemotherapy [431], where nurses, chairs, and pharmacists are involved in patient treatment, and inpatient admission planning [85], where both room-assignment and surgery scheduling are considered. In these studies, additional objectives are taken into account: workload leveling for nurses and pharmacists [431], adequate patient-room matches [85], and OT utilization [85].

Methods: algorithms [81, 85, 396], mathematical programming [81, 431], simulation [431]

Profitability Studies maximizing revenues for near-m-m have applications in healthcare and multi-project scheduling. In the healthcare application, the scheduler decides on an admission date for each accepted inpatient, accounting for the patient's expected resource consumption, access time targets, and revenue [34]. In applications for multi-project scheduling [17, 98], the authors optimize the decision which projects to accept, when to schedule their activities, and to which resources to assign these activities. 
Methods: agent-based models [17], algorithms [17, 34], dynamic programming [17, 34], game theory [17], mathematical programming [98]

Utilization Utilization-related objectives for near-m-m are considered in a study for a chemotherapy outpatient clinic [425] and a study developing a roadway reservation system [317]. In [425] all newly arrived appointment requests are assigned to time slots each week, with the objective of balancing the bed occupation over time. In [317] the reservation system decides which requests to accept and additionally negotiates the arrival times with drivers, minimizing drivers' delays while utilizing the road infrastructure efficiently.

Methods: algorithms [317], mathematical programming [425], simulation [317, $425]$

Completion times Demand management for assemble-to-order manufacturing [263] is a field where completion times are important. A demand manager decides periodically which customer orders to accept and which capacity (raw materials or modules) will be used to fulfill these. Additionally, the manager strives to keep the available-to-promise inventory at a desired level in anticipation of future customer orders.

Other applications where completion times play a role are communication via optical burst switched networks [440], and machine scheduling [476].

Methods: algorithms [440, 476], forecasting [440], mathematical programming [263], scheduling [476], simulation [440, 476]

\subsubsection{Online scheduling}

Accessibility Accessibility objectives for onl-m-m are studied in two fields: healthcare and communication. In both fields, requests consist of multiple appointments requiring multiple resource types, possibly simultaneously. A scheduler decides to which resource(s) each request should be assigned at which time. Healthcare applications are scheduling appointments for outpatient rehabilitation [63], nuclear medicine procedures [391-393], elective surgeries [500], and veterinary clinics [462]. Next to providing patients with timely access to care, several other objectives are considered: maximizing the number of patients treated [391393], minimizing the clinic's overtime [500]; limiting the number of patient visits to the clinic by combining multiple appointments on one day [63]; and synchronizing the moments at which patient treatment with various disciplines starts [63].

Communication applications are grid computing [1, 5, 106, 226, 291, 479, 496], optical burst switching [107], and multimedia applications [191, 534]. In these applications the most prominent objective is minimizing the blocking probability [1, 5, 106, 107, 191, 226, 496, 534], and other objectives are minimizing the cost of the resource assignment [5, 291, 479], minimizing sojourn times [106, 291, 479], providing timely access [107, 291, 479], and utilizing resources efficiently $[1,5,226,291,496,534]$. In addition, [1, 5, 479] compare various algorithms based on their running time. 
Methods: algorithms [1, 5, 106, 107, 191, 226, 291, 391, 392, 462, 479, 496, 500, 534], mathematical programming [5, 63, 393, 500], networks and graphs [1, 5], queueing theory [5], review [406], simulation [1, 5, 63, 106, 107, 226, 291, 391$393,462,479,496,534]$

Utilization Maximizing the system utilization while balancing the utilization over time is the objective for studies in several applications: rehabilitation outpatients requiring a sequence of physical treatments on one day [483], road users reserving a time slot to drive along a certain section of a highway [155], or users requesting execution of their application on a high performance computing system [190]. The scheduling decisions vary per application: in [483] the scheduler decides on the arrival time of a patient during the day; in [190] the scheduler provides users with insight in system utilization, to encourage them to request capacity in relatively quiet periods; and in [155] the scheduler accepts requests based on booking limits.

Methods: algorithms [190, 483], neural networks [155], simulation [483], stochastic models [483]

Completion times Minimizing completion times for onl-m-m is important for manufacturing processes in which products are not allowed to wait between the production steps, so called 'no-wait constraints' [91, 93-95, 309, 533]. Each production step has a processing window, which determines the minimum and maximum time a product spends in this step. The scheduler decides when each process step starts and how long it will take, while making sure the products can be transported between the steps.

Minimizing the completion time of each individual job without no-wait constraints plays a role in computer grids [448] and in minimizing a patient's length of stay in a hospital [344]. Focusing on the entire system rather than on individual jobs, several machine scheduling papers [96, 97, 411, 527] and a study on chemotherapy outpatient scheduling [214] have the objective of makespan minimization.

Finally, in grid computing [176, 279, 354, 463], project scheduling in a production environment [521], and designing routes for electric vehicles with swappable batteries [4], jobs have a preferred completion time and the objective is to minimize the delay over all jobs.

Methods: agent-based models [344, 521], algorithms [4, 91, 93-97, 176, 214, $279,309,354,411,448,463,527,533]$, dynamic programming [4], networks and graphs [4, 95, 448], scheduling [96, 97, 411, 463, 527], simulation [176, 309, 354, 411, 463], stochastic models [279]

\subsection{Appointment scheduling models}

In the previous sections we categorized the appointment scheduling literature based on our taxonomy and the objectives studied. In this section we review 
the suitability of different OR techniques for analyzing and/or optimizing online appointment scheduling. We focus on: the efficiency and effectiveness of the OR method; the ability of the method to incorporate realistic assumptions such as stochastic service durations; and the practical relevance of the method in solving instances of realistic size. We discuss all methods listed in Section 4.6, but focus on the methods that are actually used for the appointment scheduling; if authors for example use simulation to validate the scheduling policy they obtained using another method, this paper will not be considered for the method 'simulation' in this section. We distinguish different types of OR methods in this section, based on the type of results that can be obtained with the method: optimization methods, approximation methods, evaluation methods, and structuring/supportive methods. In the following subsections we clarify each type and discuss the OR methods of this type. See Section 2.3.1 for more mathematical details on the models.

\subsubsection{Optimization methods}

Optimization methods result in an optimal decision rule or capacity assignment with respect to a certain objective, if necessary taking restrictions on the solution space into account via constraints. Dynamic programming, mathematical programming, and graph theory approaches may be used to optimally schedule appointments, and are discussed in this subsection.

Dynamic programming Dynamic programming approaches are often used to determine an optimal policy when stochasticity in the system is taken into account. Dynamic programming is applied to: assigning a customer to the optimal (reserved) slot or resource [17, 28, 184, 187, 188, 514, 529, 545]; reserving part of the capacity for a certain customer type [23, 74, 183, 185, 275, 414, 482]; determining which slots should be offered to an arriving customer [171]; determining which customer type should be served next [198]; optimizing how many customers may be scheduled each time period [322, 376]; and order acceptance [229, 507]. In the literature, two types of dynamic programming models are applied: Markov decision theory and approximate dynamic programming models. The latter type is discussed in Section 4.4.2.

Dynamic programming models can take many characteristics of real-life problem instances into account, such as stochasticity in for example (future) arrivals and service durations. As a consequence, dynamic programming methods can be designed to closely mimic real-life systems, incorporating as many details as necessary for decision making. However, including more details often significantly increases the model's solving time and may result in an 'exploding state space', which is presumably why most papers using Markov decision theory are in the 1-1 category.

This method may be applied to obtain optimal scheduling decisions at all horizons. Solving dynamic programming models is often computationally expensive, but these models are typically only solved once, to obtain a scheduling 
policy that remains valid as long as system parameters do not change significantly. However, such optimal policies may be complicated, involving different actions for different states of the system, and could therefore be less useful for implementation in practice or decision support methods. To this end, authors often design heuristic decision policies that are based on the optimal policy.

Mathematical programming Mathematical programming is used most often for appointment scheduling, and can be applied to $\mathrm{m}-\mathrm{m}$ systems and all scheduling horizons. This approach is applied mostly at a capacity allocation horizon, for example to determine a tactical schedule $[27,29,38,39,47,50,75]$ or booking limits [2, 87]. At an online and a near-online horizon, typical problems are to find the best appointment slot for one or multiple patients $[63,70]$. Mathematical programming models can be used to optimize many different (combined) objectives, and can incorporate many details in the constraints on the solution space.

For realistic-sized instances with many integer decision variables, mathematical programming models often suffer from the 'curse of dimensionality', and the method is computationally expensive. At a capacity allocation horizon models are typically evaluated once, which is why most of the mathematical programming literature investigates this horizon. Short solving times are important on near-online and online horizons, and at these horizons mathematical programming is applied relatively often to $1-1$ and m-1 systems. To overcome the curse of dimensionality authors additionally use heuristics, either for the appointment scheduling itself, often based on the relaxation of the mathematical programming model $[29,77,89]$, or for reducing the solving time [27, 39]. Incorporating stochasticity in mathematical programming models is possible, but it makes solving the models even more computationally expensive. To this end, authors often simplify the stochastic models by only taking the average values into account $[38,39,78]$, or re-evaluate the mathematical programming model incorporating simulation results on the schedule [29].

Networks and graphs Graphs are used for optimal near-online and online appointment scheduling by constructing a directed acyclic graph that consists of nodes that represent a combination of a time slot, resource and task [1, 95, 448, 457]. The optimal schedule for all tasks of a request is identified by determining the shortest path from the first to the last task of the request. Although this approach can also be applied to capacity allocation horizons, we did not find papers using this approach.

In order to be able to construct a directed acyclic graph, the system needs to be evaluated at time slots, hence graph theory approaches are limited to discrete time systems. Graphs can be used for appointment scheduling in $\mathrm{m}-\mathrm{m}$ systems, but for realistic instances the graphs may contain many nodes and storing all nodes then becomes computationally expensive. However, the shortest path algorithm is efficient even for large graphs. Incorporating stochasticity in 
graphs is not possible, which limits the type of applications that can be modeled realistically.

\subsubsection{Approximation methods}

In this subsection we include methods that are or provide scheduling rules that are designed to rapidly provide good solutions. Approximation methods are increasingly important for real-life applications, due to their ability to solve large problem instances and/or provide solutions rapidly. We discuss algorithms, constraint programming, approximate dynamic programming, neural networks and simulation.

Algorithms Algorithms are often invoked as a practically relevant online appointment scheduling method, as they are designed to be simple and quick. Examples of online scheduling algorithms are 'assign the request to the first available time that is sufficient (first fit)', and 'assign the next customer to the resource that results in the smallest waste of capacity when you take future arrivals into account (best fit)' [401]. At the near-online scheduling horizon, examples are 'shortest remaining processing time first' and 'earliest due date first' [401]. Algorithms may also be applied at the capacity allocation level. A famous example for this horizon is the Bailey-Welch rule [26], which prescribes that at the beginning of a day one appointment slot should be overbooked to reduce server idle time in case of stochasticity in the appointment duration or no-shows.

In production scheduling applications, the performance of an online algorithm is assessed through its complexity and worst-case performance compared to the optimal offline schedule. Assessing the quality of a schedule by its worst-case performance is robust, but may be less interesting for practitioners compared to average case performance. For communication applications, short running times and low memory requirements are almost as important as the quality of the schedule. These objectives are typically conflicting, as improving the quality of the schedule requires more information about the system, which generally increases the complexity of the algorithm. Typically, algorithms that incorporate stochasticity, for example in service durations or future requests, have higher complexity compared to their deterministic counterparts, compare for example the overviews of online and semi-online scheduling algorithms provided in [24, 297].

Algorithms may be based on properties of the system or customer population, but may also be a simple, implementable approximation of a complicated policy that results from an optimization method. Certain algorithms are proven to be optimal for systems with specific characteristics [401], but most often heuristics approximate the optimal schedule or scheduling decisions. Due to their simplicity, relatively low memory requirements, and short calculation times, algorithms are often easier to implement as a decision support system in practice than other scheduling methods. 
Constraint programming We have only found one application of constraint programming [214], in which the method is used to obtain template schedules for several demand scenarios, but we expect that this method will be applied to appointment scheduling systems more often in the near future. Constraint programming is a relatively new OR method that is effective and efficient in solving large combinatorial problems, often more focused on finding a feasible solution instead of the optimal solution. Constraint programming models can cope with a wider range of constraint types compared to mathematical programming models, and are therefore more flexible and better suited for solving $\mathrm{m}-\mathrm{m}$ systems, including stochasticity, and taking future arrivals into account. As a consequence, constraint programming models may mimic practical applications better than mathematical programming models. However, as these methods are approximation methods, the solution quality may be less compared to mathematical programming approaches.

Approximate dynamic programming Dynamic programming approaches are often used to determine an optimal policy when stochasticity in the system is taken into account. One of the main disadvantages of this approach is that for many realistic problem instances the exact method suffers from the so-called 'curse of dimensionality'. The relatively new approximate dynamic programming approaches overcome this problem, as they approximate part of the Markov decision problem to reduce its state space or action space to be able to cope with larger problem instances. Approximate dynamic programming models are used to optimize the strategy of changing the batteries of electrical vehicles [4], schedule appointments with multi-skilled employees [9,33], optimize the number of admissions per day [34, 165, 225, 230,394], and to schedule appointments when each customer type has different access time requirements [189, 312, 338, 387, 433].

Similar to their exact counterparts, approximate dynamic programming models can take many characteristics of real-life problem instances into account and can be designed to closely mimic real-life systems. This method may be applied to obtain approximately optimal scheduling decisions at all horizons, and is relatively often applied to $\mathrm{m}-\mathrm{m}$ systems. Solving approximate dynamic programming models is often computationally expensive compared to other approximation methods, but these models are typically only solved once to obtain a scheduling policy that remains valid as long a the system parameters do not change significantly. Similar to policies obtained through exact dynamic programming, approximately optimal policies may be complicated, involving different actions for different states of the system, and could therefore be less useful for implementation in practice or decision support methods. To this end, authors often design heuristic decision policies that are based on the approximately optimal policy.

Neural networks Neural networks are relatively new approximation approaches that are used in the literature to accept or reject online requests of 
drivers to reserve parts of a highway for certain time-windows [155, 543]. Neural networks can be trained with the best offline solution (obtained through a different method), and learn from the effect of each decision it advises. This makes neural networks very flexible, and they can model m-m systems and any type of (future) arrival and service time distributions. Because neural networks are self-learning they are robust against changes in the parameters of the system. Compared to general algorithms and heuristics, designing a neural network is relatively complicated. Additionally, neural networks are typically more computationally expensive compared to heuristics, but much less computationally expensive compared to optimization methods.

Simulation Simulation models can incorporate any detail from practical settings that is measurable with data and can be captured in programming code. Generally, simulation models are not suitable for optimization purposes, as this would require complete enumeration of all possible solutions. However, 'simulation optimization' has gained popularity over the last two decades; this term unites a collection of heuristics that aim to determine the (approximately) optimal input parameters to maximize the output of the system. There are several simulation software packages equipped with simulation optimization toolboxes [472], which are quite efficient in finding the approximately optimal solution. However, the running time of these optimization methods depend significantly on the running time of the simulation model.

In the literature, simulation optimization is only applied to the capacity allocation horizon $[82,238,271,318,390,421,461]$, but the method may also be applied to online and near-online scheduling if the running time of the simulation model permits it. Simulation models can be applied to $\mathrm{m}-\mathrm{m}$ systems and can incorporate any arrival or service distribution, so these models can closely mimic realistic systems. Drawbacks of simulation models are that they generally require significant developing time, and are often not generalizable to other systems, even when these systems have similar characteristics.

\subsubsection{Evaluation methods}

There exist several OR methods that cannot be used for online appointment scheduling, but can be used to evaluate different scheduling policies. These OR methods are queueing theory, stochastic models, and simulation.

Queueing theory Due to the readily available results, standard queueing models are relatively quick in relating the capacity of resources to access times or blocking probabilities. Queueing models may be used to evaluate appointment systems at all scheduling horizons, and can incorporate many different probability distributions for the demand and service times. Appointment scheduling literature using this method either investigates the performance measures of an appointment system [88, 112-114, 126, 127, 136, 281, 303, 327, 510, 551], use the 
model to determine the best capacity [135, 197, 252, 283, 320, 321, 330, 535], provide customers with an estimate of the current waiting time [18, 21, 283], or determine the best customer inter-arrival times [262].

For several basic continuous time queueing models, formulas for all performance measures are available in the literature, which is why these models are often used as a reasonably good approximation for a lower bound on the necessary capacity. However, incorporating customers that make appointments requires time-varying arrival rates, and generally, for other distributions than homogeneous Poisson arrivals the formulas are hard to obtain for a continuous time queueing model. To overcome this problem relatively new approaches are to approximate the performance measures by considering the system in an asymptotic regime $[18,21,303]$ or using fluid approximations [283, 330, 535]. In systems with many servers in which customers request a relatively small part of a resource's capacity these approximations are quite accurate, but for smaller systems they are not realistic. Another drawback of continuous time queueing models is that they often investigate steady-state performance, while in practice some systems do not operate continuously long enough for the system to reach stationarity.

Discrete time models can incorporate more arrival rate distributions compared to their continuous time counterparts. However, because time is discretized customer arrivals and service completions are assumed to occur at discrete points in time, which might not be a realistic assumption.

Queueing theory is thus a useful tool for quickly determining the capacity that is approximately required in a system to achieve prescribed performance measures, but has its limitations in closely mimicking real-life systems.

Stochastic models Stochastic models are typically used to evaluate appointment systems at a capacity allocation horizon, but are also used for online scheduling horizons $[258,365,483,525,546]$. Papers using stochastic models invoke either convolutions [15, 256, 318, 407, 408, 470, 493] or Markov chains $[129,164,258,365,438,483,505,525,536,546]$. Given an appointment schedule and the service time distribution, the probability distribution of the waiting time or overtime can be obtained by taking convolutions of the service time distributions. Markov chains consist of a set of states and transition probabilities between these states, which depend on the scheduling rule. The stationary distribution of a Markov chain can be used to obtain for example the waiting time or access time distribution.

Stochastic models are applied most often to healthcare applications, to balance patients' waiting times with the idle time and overtime of practitioners at outpatient clinics. The models may be applied to any scheduling horizon, but are most often applied at a capacity allocation horizon. Although in theory stochastic models may be used to analyze $\mathrm{m}-\mathrm{m}$ systems, the rapidly growing state space or tractability of the convolutions generally only allows for analyzing 1-1 systems. Both convolutions and Markov chains require discrete event points between which the system is assumed to be in one state, but these points may be 
at stochastic distances of each other $[505,525]$. In order to reduce the complexity of the models, appointment systems are often analyzed in discrete time, i.e., with equal distances between the event points. As appointment systems often have fixed appointment slot lengths, the discrete-time assumption is generally quite realistic.

Simulation In appointment scheduling literature, simulation is typically used to evaluate appointment scheduling rules that are obtained with other methods. Additionally, when the number of possible solutions is limited simulation is used to compare all possible schedules or the most promising schedules [159, 266], or to investigate additional performance measures that cannot be obtained with analytic models [15, 84, 438]. All applications of simulation models as evaluation models are at the capacity allocation horizon, which is presumably because the trial-and-error solution approach typically requires too long running times for online and near-online scheduling. Simulation can mimic realistic systems as closely as necessary, as they can incorporate any detail from practical settings that is measurable with data and can be captured in programming code. Drawbacks of simulation models are that they generally require significant developing time, and are often not generalizable to other systems, even when these systems have similar characteristics.

\subsubsection{Structuring/supportive methods}

Some OR methods listed in Section 4.6 cannot be used to obtain online scheduling policies, but are methods to simplify the system or support the decision making. Agent-based models, forecasting, game theory, and scheduling are such types of methods, and will be discussed in the following.

Agent-based models Typically, agents in the system represent either a customer or a resource manager. Customer agents try to optimize all appointments from a customer-viewpoint, based on the appointment slots that resource agents offer. Resource agents may compete with others in an auction to be allowed to serve the next customer $[17,357]$, or base the price of their services on heuristic rules $[434,499,502]$ or a forecast of future demand [467]. In [138] mathematical programming is used to set booking limits for all agents, and agents decide whether to accept or reject customers based on the request characteristics. After the customer is assigned to a resource agent, often the resource agent individually schedules the customer's request, but agents may also cooperate in determining the best schedule [434]. The actual resource schedule is often obtained using a scheduling heuristic.

Agent-based models decompose scheduling problems into several subproblems. This decomposition makes solving the scheduling problem smaller and thus easier to solve. Therefore, agent-based methods are suitable to apply to m-m systems, even for large instances of real-life applications. However, 
decomposing the problem results in locally optimal schedules, which may be suboptimal globally. The actual appointment scheduling decision is not solved by the agent-based model itself, which is why the efficiency, effectiveness, flexibility, and practical relevance of this approach depends on the scheduling method used by the agents. Agent-based models can incorporate many different scheduling methods, often only restricted in the scheduling method's solution time for practical applications.

Forecasting Forecasting methods, such as regression theory or time series analysis, are typically used to support the appointment scheduling decision, for example by forecasting the demand to reveal times with relatively low or high workload [110, 467], or by estimating processing times [440]. In [84] forecasting is used to evaluate customer waiting time and server idle time for different appointment schedules. The results of forecasting methods may be fed into an (approximate) optimization model [84], or incorporated in a scheduling heuristic [110, 440, 467]. For most realistic systems it is necessary to incorporate stochasticity in a model in order to achieve a close fit between the model and reality, which can be achieved using forecasting methods or assuming that random variables follow a certain probability distribution, such as the Poisson distribution. Forecasting methods are not often used in the literature, because probability distributions tend to be easier to incorporate into mathematical models.

Game theory Game theory approaches are used to analyze customer behavior in a system in which customers may join the queue or pay to make an appointment at a specific time [452]. Non-cooperative game theory is effective in analyzing the effect of different cost structures for making appointments and informationsharing policies in an appointment system, strategies used to stimulate desired customer behavior or maximize the server's profit. Typically, it is assumed that each customer has a certain utility function that reflects how happy this customer is with a given service level, such as waiting time or service costs. Additionally, all customers follow some (stochastic) decision strategy, which prescribes which action the customer will take to maximize its utility for each state of the system. Game theory analyzes the 'equilibria of the system', in which it is optimal for all customers to follow the same, equilibrium-specific strategy. For these equilibria the performance measures of the system, such as profit, can be calculated. Game theory can be applied to all scheduling horizons, but has so far only been applied at the online horizon [452]. Different arrival and service time distributions can be incorporated in this approach, and the method is relatively computationally inexpensive.

Scheduling Scheduling literature investigates the performance of exact algorithms and heuristics that assign jobs to machines, often with the objective to minimize the makespan. After a job is assigned to a machine, each machine typically processes the jobs in order of arrival. As each job's processing time is 
assumed to be known after the assignment to a machine, assigning a job to a machine is the same as making an appointment. The quality of online scheduling algorithms is judged by their competitive ratios (see Section 4.2.3). The scheduling literature cannot be applied to the capacity allocation horizon, but is applied to online and near-online scheduling horizons. Scheduling literature always refers to a standard framework to describe each problem's characteristics in a condense way. Due to this framework and the fact that all scheduling literature applies the same performance measure, the performance of different algorithms for different systems can easily be compared.

A drawback of scheduling literature for practical appointment scheduling systems, is that stochasticity in the service durations cannot be taken into account in the heuristics, as a customer could not obtain its appointment time anymore in such systems. Furthermore, jobs are always processed in order of arrival, which also limits the practical relevance of scheduling methods. Additionally, as previously stated, assessing the quality of a schedule by its worst-case performance is robust, but may be less interesting for practitioners compared to the average case performance.

\subsubsection{Conclusion}

All appointment scheduling models have their advantages and drawbacks, and the characteristics and/or goals of the application may make one method more favorable than others. At a capacity allocation horizon, optimization methods are useful, and dynamic programming is most suitable for including stochasticity. For online scheduling problems, stochastic models or neural networks are useful when stochasticity has to be taken into account, otherwise algorithms are most useful as they provide solutions rapidly. At the near-online horizon, smaller instances can be solved using optimization methods, but when the running time of these methods is impractically long, algorithms or neural networks can provide approximate solutions.

The robustness of an appointment schedule or scheduling policy is important when it is implemented in practice. As dynamic programming approaches incorporate stochasticity, this optimization approach provides the most robust solutions. Due to the self-learning effect, neural networks provide the most robust approximate solutions even when some characteristics of the system change.

As the problems OR researchers want to solve tend to get more realistic and (thus) bigger, exact methods should be made more efficient in the near future to solve larger systems exactly. Approximation methods will become more important in the OR literature, as such methods are promising for rapidly delivering solutions of relatively good quality.

\subsection{Discussion}

Online appointment scheduling has received increasing attention from researchers over the last decades (see Figure 4.2). Different application areas focus on dif- 
Figure 4.2 Number of online appointment scheduling papers published per year (1990-2014).

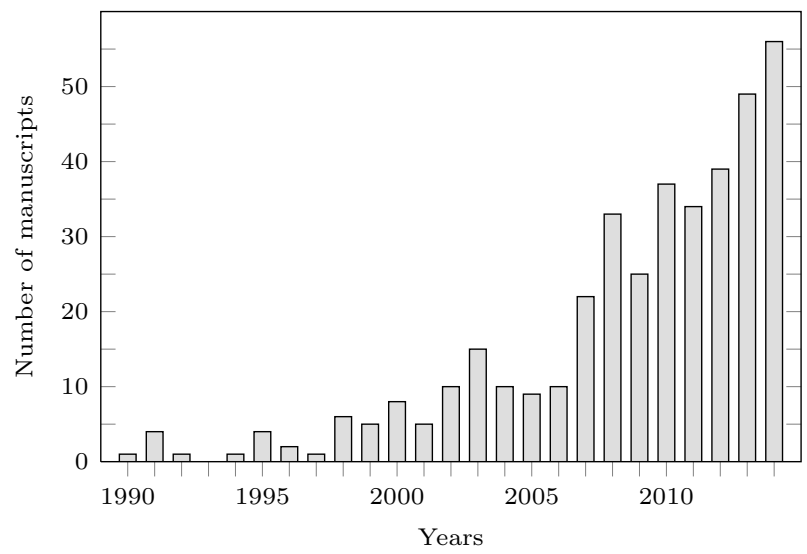

ferent modeling assumptions and goals. For healthcare applications, access time norms and the effects of server consistency and no-shows are important. Reliability and throughput are the main objectives for communication and transportation applications, while utilization and revenue are more important in service industries, and completion times are essential in production environments. In all application areas, there is a continuous trade-off between customer-friendliness, for example, short access and waiting times, and system efficiency, for example, little idle time and overtime of the server.

Almost half of the manuscripts in this review consider online scheduling, $35 \%$ consider capacity allocation, and 17\% near-online scheduling. From our review we identify several categories of the taxonomy and application areas that are not often studied in the literature (see also Table 4.1). Below, we first discuss why certain categories of our taxonomy have a lower share of citations than appear in literature, and then identify several promising directions for future research. Tables and graphs supporting the observations in this section can be found in the supplementary material (www.utwente.nl/choir/bibliography.xlsx).

In our review, the majority of manuscripts consider a utilization (39\%) or an accessibility (35\%) objective; completion times (19\%) and profitability (7\%) receive less attention. Manuscripts studying profitability are often excluded because revenue management methods are applied. Due to the same reason, service $(1 \%)$ and transportation applications $(7 \%)$ receive less attention. This mainly affects the capacity allocation level, as revenue management typically optimizes booking limits. Completion time objectives also receive less attention. Most of these manuscripts focus on machine scheduling, recently reviewed in $[12,296,454,469]$. As a consequence, also production applications appear less often in our review (15\%). Healthcare $(51 \%)$ dominates our review, followed by communication (19\%).

Some objectives defined in this review are related. Balancing utilization across 
machines and minimizing system completion times are related, as both imply a schedule that minimizes the maximum makespan. Minimizing the blocking probability for a given customer mix with equal appointment lengths maximizes utilization. Additionally, when all servers work at equal speed and processing times are deterministic, the access time and (individual) completion time objectives are equivalent.

From our review, we identify several promising directions for future research. Most of the online appointment scheduling literature focuses on 1-1 (for an explanation of the abbreviations, see Section 4.2.2) systems (60\%), or m-m systems (22\%); 1-m and $\mathrm{m}-1$ systems have received little attention. One exception is onl-1-m, which mainly has applications in transmitting data packets via computer networks. In the field of communication, scheduling by means of heuristics is common practice, as it requires fast procedures that use little computational power. Studying cap-1-m could provide communication applications with scheduling strategies, decreasing the online computation time and power, and possibly also improving solution quality.

Near-online scheduling is the least studied scheduling horizon in our taxonomy. In the literature, offline scheduling is typically used to assess the quality of online scheduling algorithms (c.f., [296, 393]). However, pragmatically, nearonline is much closer to online scheduling than offline scheduling, and easier to implement. Near-online scheduling policies should in theory outperform online ones, as the scheduler is better informed. Therefore, future research on nearonline scheduling and its comparison to online scheduling would be valuable for advising service providers on which of these two policies to use.

In online and near-online scheduling, most studies either consider a problem restricted to one day and decide on optimal appointment times, or consider a longer planning horizon and decide only on the future day to assign an appointment request to. Studies in the latter category commonly limit the total demand that can be scheduled on one day to the total capacity offered on that day. However, in settings with multiple servers and various customers' appointment lengths (c.f., [188, 433]), constructing a schedule from this solution in which each service is uninterrupted might be impossible. Studies that do decide on optimal appointment times usually consider a problem restricted to one specific day, not incorporating the varying resource availability on different days, customers with different access time targets, or customers who require a series of appointments on multiple days. Simultaneously deciding on both day and time of appointments is essential in many application areas, but studied in few papers [81, 396, 425, 429], making it a promising research challenge. As such studies are closer to reality, the research results will better translate into practice.

Online and near-online scheduling could significantly benefit from anticipating future appointment requests when scheduling the current appointment(s), c.f., $[386,393]$, but many studies develop myopic approaches not taking the future into account. [393] shows that when anticipating the future, more customers can be served with the same capacity, while customers receive quicker access and 
customers' preferences are satisfied more often.

The capacity allocation level in our taxonomy is dominated by healthcare applications (89\% of manuscripts). Most of this research focuses on making blueprint schedules, aiming to guarantee sufficient resources for urgent patients after all appointments with longer planning horizons have been scheduled. Other application areas serving customers with different planning horizons are, for example, communication networks with (long-term) data transfers and regular Internet traffic (see the paragraph on completion times in Section 4.3.2.2), or service mechanics with maintenance activities and emergency incidents [130]. Such application areas might benefit from applying, possibly with some adjustments, the methods that have been proven to be valuable for healthcare.

In healthcare, the predominant objectives studied are accessibility and utilization, but additional gains may be achieved by minimizing (individual) completion times. Many one-day diagnostic or therapeutic programs are currently initiated for outpatients, where the cumulative waiting time for patients during such a day is often overlooked. For inpatients, decreasing the time between appointments leads to a decrease in their length of stay [344]. Both patient-friendliness and system efficiency (e.g., decreasing the use of overtime [214]) could benefit from studying completion time objectives in healthcare.

Production is the field where completion time objectives are currently studied most, mainly at the online scheduling level. The near-online level gradually receives increasing attention in machine scheduling. However, we have found only one paper studying a completion time objective at the capacity allocation level. As several studies on accessibility and utilization objectives have shown how (near-)online scheduling benefits from methodologies developed for capacity allocation, studying completion time objectives for this scheduling horizon is a promising direction for future research.

Current societal developments give rise to new research directions in online appointment scheduling. For transportation applications, we have identified a number of papers on scheduling vehicles on highways [155, 170, 316, 317], at intersections [148, 499], or in urban areas [543]. The introduction of self-driving vehicles brings a considerable increase in (central) control of such systems, opening up possibilities for more sophisticated scheduling and implementation of the results. Additionally, the introduction of electric vehicles yields a wealth of future research questions [4], for example relating to the location and capacity of recharging points, and online assignment of vehicles to these points. Such questions frequently involve a trade-off between social and individual optima.

Another societal development is the increasing ability for customers to schedule their own appointments via Internet. Research on all scheduling horizons could advance this development, increasing both customer-friendliness and system efficiency. A promising research question is which appointment slots a particular customer should be able to choose from, given his specific characteristics, aiming for the best solution for the global system.

Concluding, in this review we have introduced a taxonomy for online ap- 
pointment scheduling, provided a structured overview of the literature in each category of this taxonomy, and identified opportunities for future research. The literature was reviewed without restriction to any application area. Based on the manuscripts found, we have identified the following application areas: communication, healthcare, production, service, and transportation. This review aims to stimulate mutual interchange of research results from different application areas, advancing the field of online appointment scheduling.

\subsection{Appendix}

This appendix provides a short description of each of the operations research methodologies considered in this chapter. See Section 2.3.1 for more mathematical details on the models.

Agent-based models. ABMs (also multiple-agent systems) mimic the interactions of autonomous agents to assess their effects on the global system. ABMs, which are typically used in simulation programs, usually comprise of numerous agents, decision-making heuristics, learning rules, interaction topology, and a non-agent central environment [332].

Algorithms. Any procedures or formulas for solving an optimization problem. We distinguish exact algorithms and heuristics; exact algorithms return an optimal solution, while heuristics are designed to approximate optimal solutions fast [519].

Constraint programming. Constraint programming is a paradigm in which the user states relations (constraints) among decision variables. Heuristic constraint solvers are used to find an assignment to all decision variables that satisfies all constraints [424].

Decision analysis. Methods for scoping a decision-making problem: clearly representing it, decomposing it into elements, establishing criteria, evaluating the options, and recommending a decision to a decision maker [174].

Dynamic programming. A set of approaches for solving sequential decision problems: deterministic, stochastic, and approximate dynamic programming; and Markov decision theory $[402,405,519]$.

Forecasting. The use of a model to predict future values of random variables based on historical data. Two commonly used techniques are regression analysis and time series analysis $[216,519]$.

Game theory. A mathematical method for decision making in which a cooperative or non-cooperative competitive situation is analyzed to determine the optimal strategy (including bidding and auctions) [366, 395].

Mathematical programming. A collection of tools for solving linear, nonlinear, or stochastic optimization models consisting of an objective function, a set of parameters and decision variables, and a set of constraints that restrict the decision variables [519]. 
Networks and graphs. Networks and graphs are sets of objects (nodes, vertices) that are connected by links (edges). This field includes for example the vehicle routing and maximum spanning tree problem [54, 372].

Neural networks. An artificial neural network (ANN) is a paradigm that can be used to extract patterns and detect trends in large datasets. An ANN is built for a specific application, and learns from new data input [151, 231].

Queueing theory. Mathematical models for (networks of) waiting lines, or queues. Typical input for these models is the arrival process of customers, and the service requirement distribution, discipline, and capacity [423, 519].

Scheduling. The process of assigning jobs to one or multiple machine(s) in such a way that a given performance measure is optimized. Typical objectives are minimizing the latest completion time (makespan) or the number of machines required [401].

Simulation. Mimicking a real-world system as it evolves over time. The 'simulation model' is evaluated by a computer to generate representative samples of the performance measure of interest [519].

Stochastic models. Mathematical models that mimic the random evolution of a system. We distinguish three types of stochastic models: Markov process, renewal processes, and (general) stochastic processes [423]. 


\section{Blocking probabilities in queues with advance reservation}

\subsection{Introduction}

In a reservation system, a job can reserve the system resource(s) a certain notice time in advance. The model has a wide range of applications besides healthcare, for example conference calls in telecommunication networks [418] and optical burst switching networks [258]. Related topics include virtual queueing [283]; advance reservation algorithms, cf. [73]; admission control algorithms, cf. [199, 507]; and routing in reservation systems, cf. [92].

Despite their simple structure, queueing systems with reservation appear to be hard to analyze. In particular, there is no a priori intuition on the consequences of advance reservation on blocking probabilities. This question is addressed in the present chapter.

The discrete time equivalent of reservation models has been addressed in the literature, see $[113,160,258,311,506,546]$ for different analytic approaches. The blocking probabilities in the discrete time systems appear easier to analyze because, for each time slot, one only needs to check whether the system is occupied or idle in this slot [113]. Nevertheless, it is hard to deduce from the balance equations or limiting distribution whether the blocking probability in these systems is increased or decreased as a result of reservations. Moreover, this question has been rarely addressed. Interesting numerical examples in this direction are provided in [113]. In particular, the authors notice that reservations can both increase or decrease the utilization of the server.

Literature on continuous time reservation models is sparse. Roberts and Liao [418] give an approximation of the arrival process of reservation calls to a telecommunication system, and derive the required capacity for achieving prescribed performance levels. Maillardet and Taylor [337] obtained the stationary distribution of the system content in case of infinite number of servers. Levi and Shi [303], Lu and Radovanović [324] approximate the blocking probability in a system with $c$ servers by the probability that more than $c$ jobs are present in the infinite capacity system. Coffman Jr. et al. [113] analytically obtain the fraction of time the system resource is occupied in a number of special cases, including 'short notice' (notice time strictly smaller than the service time) and a discrete 
notice time. This work is most related to the analysis in this chapter because the blocking probability can be readily derived from the server utilization.

In this chapter we extend the above mentioned results by providing insights in the impact of advance reservation on blocking probabilities, both analytically and numerically. We address the intriguing question of how reservation affects the blocking probabilities in the system. We show that in some systems, such as the systems with deterministic notice time, reservation can only increase the blocking probabilities. On the other hand, in systems with bi-modal notice time or exponentially distributed service time, the effect on blocking probabilities depends on the system's parameters. We also notice that systems in which reservations decrease the blocking probability, shorter jobs are favored over longer jobs. Thus, the impact of advance reservation can be significant on the system's performance and job selection.

\subsection{The model and special cases}

We study the reservation loss system with Poisson $(\lambda)$ arrivals, $c$ servers, service time $S$, and notice time $R$. Job $i, i=1,2, \ldots$, arrives at time $t_{i}$ and requests a service time $S_{i}$ that starts after the notice time $R_{i}$, where $S_{i}$ 's and $R_{i}$ 's are independent copies of $S$ and $R$, respectively. Thus, job $i$ claims the service interval $\left(t_{i}+R_{i}, t_{i}+R_{i}+S_{i}\right.$ ]. If there are less than $c$ servers occupied on this entire interval, then the job is accepted. Otherwise, the job is blocked and lost. Accepted jobs that are in the system but are not yet served are 'in reservation'.

Figure 5.1 Possible realization of reservation system for $c=1$.

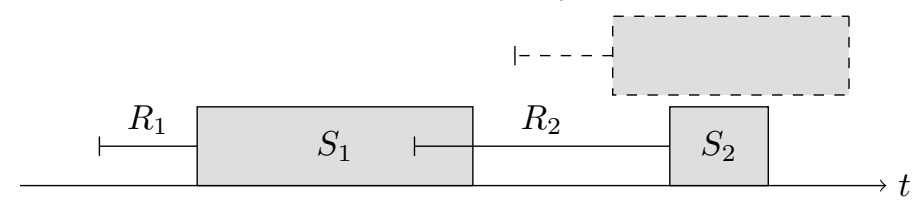

Figure 5.1 shows a possible realization of the reservation system with $c=1$. Here the requested service intervals of the arriving jobs are represented by a rectangle and the notice time is denoted by a line. The dashed job is blocked, because in its requested service interval there is another job that arrived earlier. Note that the dashed job is blocked although its service would have started earlier than the service of job 2 , due to the advance reservation.

Following Maillardet and Taylor [337], the state of this network with $n$ jobs at time $t$ is denoted by $(t, n, \mathbf{r}, \mathbf{s})$, with $\mathbf{r}=\left(r_{1}^{r}, \ldots, r_{n}^{r}\right), \mathbf{s}=\left(s_{1}, \ldots, s_{n}\right), r_{k}^{r}$ the residual notice time and $s_{k}$ the service time for job $k, k=1, \ldots, n$. Here $s_{k}>0$ and $r_{k}^{r}>-s_{k}$, where $r_{k}^{r}$ becomes negative once the service of job $k$ starts. An accepted job is allocated with equal probability to one of the positions $1, \ldots, n$. When an arriving job is allocated to position $k$, jobs $k, \ldots, n$ shift to positions $k+1, \ldots, n+1$. When a job at position $k$ departs, jobs $k+1, \ldots, n$ shift to 
positions $k, \ldots, n-1$.

State $(t, n, \mathbf{r}, \mathbf{s})$ is feasible if $\sum_{k=1}^{n} \mathbb{1}\left\{-s_{k} \leq r_{k}^{r}-\tau<0\right\} \leq c \forall \tau \geq 0$, with $\mathbb{1}\{x\}$ the indicator of event $x$, since there are only $c$ system servers and all jobs require exactly one server. The state space of this model is:

$$
\begin{aligned}
\mathcal{S}=\{ & (t, n, \mathbf{r}, \mathbf{s}) \mid \mathbf{r}, \mathbf{s} \in \mathbb{R}^{n}, n \in \mathbb{Z}^{+}, t \in \mathbb{R}, s_{k}>0, r_{k}^{r}>-s_{k}, \forall \tau \geq 0: \\
& \left.\sum_{k=1}^{n} \mathbb{1}\left\{-s_{k} \leq r_{k}^{r}-\tau<0\right\} \leq c\right\} .
\end{aligned}
$$

Observe that by introducing advance reservation, jobs are not served in order of arrival. Moreover, in general, infinitely many jobs can be in the system. Apparently, this makes the problem of obtaining an analytical expression for the blocking probability intractable, except for some special cases. This section presents analytical results for the stationary distribution and blocking probability. We first provide a system of partial differential equations for the stationary density, which appear to be intractable for the general reservation model. For the special case with an unlimited number of servers, the solution is provided in section 5.2.1. In section 5.2.2, the single server system with deterministic notice and service times is solved using a renewal argument.

\subsubsection{The general model}

Let $\mathbf{x} \backslash x_{i}$ denote that $x_{i}$ is removed from the $i^{\text {th }}$ location of vector $\mathbf{x}$ and $\mathbf{x} \cup x_{i}$ that $x_{i}$ is added to location $i$ in vector $\mathbf{x}$, and all indices are updated accordingly. Furthermore, let $b_{R}(t), B_{R}(t)$ and $b_{S}(t), B_{S}(t)$ the density functions and distributions of $R$ and $S$, respectively. Following Maillardet and Taylor [337] in the analysis for the infinite server case, let $\pi(t, n, \mathbf{r}, \mathbf{s})$ denote the density of state $(t, n, \mathbf{r}, \mathbf{s})$. The limiting density $\pi(n, \mathbf{r}, \mathbf{s})=\lim _{t \rightarrow \infty} \pi(t, n, \mathbf{r}, \mathbf{s})$ exists [360] and is obtained as a unique solution of the following system of partial differential equations. For non-boundary states, i.e., there is no moment in time that either all servers are occupied for this state, or that the system is empty, $\pi(n, \mathbf{r}, \mathbf{s})$ satisfies:

$$
\begin{aligned}
\sum_{i=1}^{n} \frac{\partial \pi(n, \mathbf{r}, \mathbf{s})}{\partial r_{i}^{r}}=\lambda & \pi(n, \mathbf{r}, \mathbf{s})-\frac{\lambda}{n} \sum_{i=1}^{n} \pi\left(n-1, \mathbf{r} \backslash r_{i}^{r}, \mathbf{s} \backslash s_{i}\right) b_{R}\left(r_{i}^{r}\right) b_{S}\left(s_{i}\right) \\
& -\sum_{i=1}^{n+1} \int_{0}^{\infty} \pi\left(n+1, \mathbf{r} \cup-z_{i}, \mathbf{s} \cup z_{i}\right) \mathrm{d} z_{i} .
\end{aligned}
$$

On the boundaries, for $n=0$ :

$$
\lambda \pi(0)=\int_{0}^{\infty} \pi\left(1,-z_{i}, z_{i}\right) \mathrm{d} z_{i},
$$


and for $r_{i}^{r}<0 \forall i$ and $n<c$ :

$$
\sum_{i=1}^{n} \frac{\partial \pi(n, \mathbf{r}, \mathbf{s})}{\partial r_{i}^{r}}=\lambda \pi(n, \mathbf{r}, \mathbf{s})-\sum_{i=1}^{n+1} \int_{0}^{\infty} \pi\left(n+1, \mathbf{r} \cup-z_{i}, \mathbf{s} \cup z_{i}\right) \mathrm{d} z_{i},
$$

and for $c$ jobs with $r_{i}^{r}<0$, i.e., $c$ jobs in service, and $n \geq c$ :

$$
\begin{aligned}
\sum_{i=1}^{n} \frac{\partial \pi(n, \mathbf{r}, \mathbf{s})}{\partial r_{i}^{r}}=\lambda & P\{\operatorname{arriving} \text { job is accepted }\} \pi(n, \mathbf{r}, \mathbf{s}) \\
& -\frac{\lambda}{n} \sum_{i=1}^{n} \pi\left(n, \mathbf{r} \backslash r_{i}^{r}, \mathbf{s} \backslash s_{i}\right) b_{R}\left(r_{i}^{r}\right) b_{S}\left(s_{i}\right) .
\end{aligned}
$$

Note that an expression for the blocking probability, $1-P$ \{arriving job is accepted $\}$, requires transient analysis of the system as it involves a time interval $\left(t, t+\max _{1 \leq k \leq n} s_{k}\right)$, see (5.1). For two special cases, the stationary density can be obtained in closed form from (5.2), (5.3), (5.4) and (5.5): the case $R=0$ and the case with unlimited capacity.

When all arriving jobs have zero notice time, i.e. $R=0$, the reservation model reduces to the Erlang loss model, with stationary density:

$$
\pi\left(s_{1}, \ldots, s_{n}\right)=\frac{\frac{\lambda^{n}}{n !} \prod_{i=1}^{n}\left(1-B_{S}\left(s_{i}\right)\right)}{\sum_{i=0}^{c}(\lambda \mathbb{E}[S])^{i} / i !} .
$$

The blocking probability for the Erlang loss model is:

$$
P^{b}(c)=\frac{(\lambda \mathbb{E}[S])^{c} / c !}{\sum_{i=0}^{c}(\lambda \mathbb{E}[S])^{i} / i !} .
$$

For the reservation model with an infinite number of servers, the system of differential equations reduces to (5.2) and (5.3). The stationary density is ([337]):

$$
\pi(n, \mathbf{r}, \mathbf{s})=e^{-\lambda(\mathbb{E}[R]+\mathbb{E}[S])} \frac{\lambda^{n}}{n !}\left[\prod_{k: r_{k}^{r}>0}\left(1-B_{R}\left(r_{k}^{r}\right)\right)\right]\left[\prod_{k=1}^{n} b_{S}\left(s_{k}\right)\right] .
$$

Unlike these two special cases, the stationary density is generally not of productform, which is argued below.

\subsubsection{Deterministic short notice}

To enhance readability, let $P_{0}$ denote the blocking probability in an $M / G / c / c$ queue and $P_{R}$ the blocking probability in a $c$-server reservation system. Consider a system with one server, where $R$ and $S$ are deterministic and $R<S$. Then, at most one job can be in reservation, and we can obtain the entire stationary distribution using a renewal argument. There are four possible situations at a 
certain time $t$ : (a) the system is empty; (b) one job in reservation; (c) one job in service; and (d) one job in reservation and one job in service.

Let $I_{j}$ the idle time between the services of jobs $j-1$ and $j$, then $I_{j}$ is exponential with parameter $\lambda$. Let $X_{t}$ be a two-dimensional vector that equals $\left(n_{R}, n_{S}\right)$ if there are $n_{R}$ jobs in reservation and $n_{S}$ jobs in service at time $t$. Then $X_{t}$ is a regenerative process, with regeneration moments at service completions where jobs leave an empty system. After each service completion, the regeneration cycle ends with probability $p=e^{-\lambda R}$. Therefore, the number of jobs in a cycle is geometrically distributed with mean $e^{\lambda R}$. Each job contributes its service time and an idle time to the expected cycle length, so the expected cycle length is $\left(S+\lambda^{-1}\right) e^{\lambda R}$.

Possible sample paths are given in Figure 5.2, where a sample path for each possible situation in this system is depicted. Let $P_{(c)}(y)$ be the stationary

Figure 5.2 Reservation system in states (a), (b), (c) and (d).

(a):

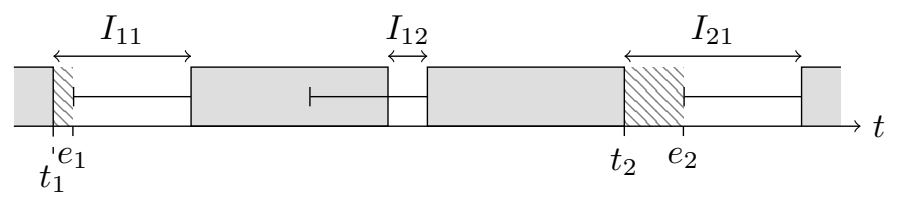

(b):

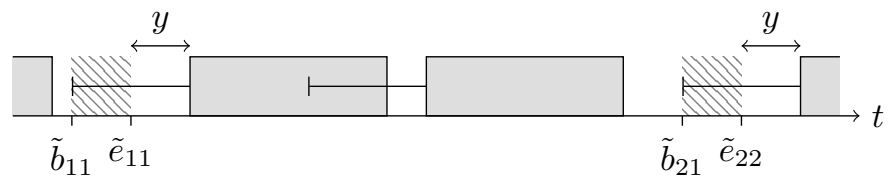

(c):

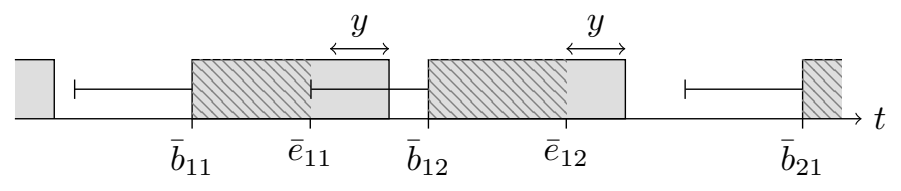

(d):

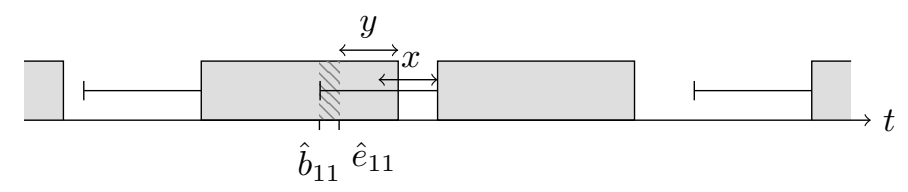

probability of having one job in service with residual service time greater than $y$. For $y>0$, define $\left[\bar{b}_{i j} ; \bar{e}_{i j}\right]$ to be the interval in which the $j^{\text {th }}$ job of the $i^{\text {th }}$ cycle has residual service time greater than $y$ and there is no other job in the system; see Figure $5.2(\mathrm{c})$, where the area of interest is marked by a dark gray pattern. We obtain $P_{(c)}(y)$ by considering the average length of interval $\left[\bar{b}_{i j} ; \bar{e}_{i j}\right]$ for each job. Note that the intervals are i.i.d. and the length of interval $\left[\bar{b}_{i j} ; \bar{e}_{i j}\right]$ depends on $I$ : it is $S-(R-I)$ if $I+y<R$, and $S-y$ otherwise. Conditioning on $I$, for 
arbitrary $i, j$ :

$$
\begin{aligned}
& \mathbb{E}\left[\bar{e}_{i j}-\bar{b}_{i j}\right]= \\
& \int_{t_{i}}^{t_{i+1}} \mathbb{1}\left\{X_{t}=(0,1), j \text { is in service and } r_{j}^{r}>-s_{j}+y\right\} \mathrm{d} t \\
& =\int_{0}^{\max \{R-y, 0\}}(S-R+x) \lambda e^{-\lambda x} \mathrm{~d} x+\int_{\max \{R-y, 0\}}^{\infty}(S-y) \lambda e^{-\lambda x} \mathrm{~d} x \\
& = \begin{cases}S-R-\lambda^{-1}\left(e^{-\lambda(R-y)}-1\right) & \text { for } 0<y<R \\
S-y & \text { for } 0<R \leq y .\end{cases}
\end{aligned}
$$

Given the mean cycle length and mean number of jobs per cycle, we obtain:

$$
P_{(c)}(y)= \begin{cases}\frac{S-R-\lambda^{-1}\left(e^{-\lambda(R-y)}-1\right)}{S-y} S+\lambda^{-1} & \text { for } 0<y<R \\ \frac{S+\lambda^{-1}}{} & \text { for } 0<R \leq y\end{cases}
$$

Furthermore, in the stationary state, let $P_{(a)}$ be the probability of an empty system; $P_{(b)}(y)$ the probability of one job in reservation with residual notice time greater than $y$; and $P_{(d)}(x, y)$ the probability of one job in reservation with residual notice time greater than $x$ and one job in service with residual service time greater than $y$.

The distribution for states (a), (b) and (d) is obtained in a similar way. For the distribution $P_{(a)}:=\pi(0)$, we determine the average length of interval $\left[t_{i} ; e_{i}\right]$; see Figure $5.2(\mathrm{a})$. Here $e_{i}$ denotes the end of idle period $i$. Define $\left[\tilde{b}_{i j} ; \tilde{e}_{i j}\right]$ the interval in which only job $j$ is in reservation in cycle $i$ and job $j$ has residual reservation time greater than $y$. The distribution $P_{(b)}(y):=\int_{y}^{R} \pi(1, x, S) \mathrm{d} x$, $y>0$, is obtained from the average length of interval $\left[\tilde{b}_{i j} ; \tilde{e}_{i j}\right]$ per job $j$ in cycle $i$, which exists for a job only if $I \geq y$; see Figure $5.2(\mathrm{~b})$. Define $\left[\hat{b}_{i j} ; \hat{e}_{i j}\right]$ the interval in which the $j^{\text {th }}$ job of cycle $i$ has residual service time greater than $y$ and the $(j+1)^{\text {th }}$ job of cycle $i$ has residual reservation time greater than $x$. Then $P_{(d)}(x, y):=\int_{0}^{S-y} \int_{x}^{R} \pi(2, w, S,-z, S) \mathrm{d} w \mathrm{~d} z, x, y>0$, is derived from the average length of interval $\left[\hat{b}_{i j} ; \hat{e}_{i j}\right]$ for each job $j$ in cycle $i$, which is of length $R-I-y$ if $I+y>x$ and $R-x$ else; see Figure $5.2(\mathrm{~d})$.

These stationary probabilities for states (a), (b) and (d) are readily obtained by conditioning on the $I_{j}$ 's:

$$
P_{(a)}=\frac{\lambda^{-1}}{\left(S+\lambda^{-1}\right) e^{\lambda R}},
$$




$$
\begin{aligned}
& P_{(b)}(y)=\frac{\lambda^{-1}\left(e^{-\lambda y}-e^{-\lambda R}\right)}{S+\lambda^{-1}} \quad \text { for } y>0 \\
& P_{(d)}(x, y)= \begin{cases}\frac{R-x-\lambda^{-1}\left(e^{-\lambda(x-y)}-e^{-\lambda(R-y)}\right)}{S+\lambda^{-1}} & \text { for } 0<y \leq x<R, \\
\frac{R-y-\lambda^{-1}\left(1-e^{-\lambda(R-y)}\right)}{S+\lambda^{-1}} & \text { for } 0<x<y<R .\end{cases}
\end{aligned}
$$

The stationary distribution is completely specified by expressions (5.6), (5.7), (5.8) and (5.9).

In the reservation system, an arriving job is blocked in one of the following three cases: either there is a job in reservation, or there are two jobs in the system, or there is a job in service with residual service time greater than $R$. Therefore:

$$
P_{R}=P_{(b)}\left((0)^{+}\right)+P_{(c)}(R)+P_{(d)}\left((0)^{+},(0)^{+}\right),
$$

with $(0)^{+}$the limit to 0 from above. Direct substitution shows that this is equal to the blocking probability in the $M / D / 1 / 1$ queue. This is intuitive, since the deterministic notice time results in an $M / D / 1 / 1$ queue in which the arrival process is delayed by a constant $R$.

This special case of the reservation model is interesting because it clearly demonstrates that, in general, reservation systems do not have a productform stationary distribution. Indeed, note that $P\{$ Nobody in service $\} \times$ $P\{$ Nobody in reservation $\} \neq P_{(a)}$. Hence, the stationary distribution for the reservation system and the blocking probability $P_{R}$ can be obtained in closedform only in special cases.

Note that the above analysis holds for generally distributed $R$ and $S$ with $P(R<S)=1$, but since this would not provide additional insight, this result is omitted. Instead, we obtain the blocking probability for a discrete notice time distribution in closed form.

\subsubsection{Discrete notice time}

Consider the special single server case where all jobs have deterministic service time $S$ and a fraction $q$ of the jobs have stochastic notice time $R$ with $P(k S<$ $R<(k+1) S)=1$ for an integer $k>0$. Then, the probability that the server is occupied can be obtained by adapting the argument in [113, Section 2.3], and is equal to:

$$
\frac{S}{\mathbb{E}[I]+S}, \quad \text { where } \quad \mathbb{E}[I]=\frac{1-(1-q)(1+\lambda q S) e^{-\lambda q S}}{\lambda q} .
$$

From this it follows that the server finishes jobs at rate $(\mathbb{E}[I]+S)^{-1}$. Since the arrival rate is $\lambda$, we obtain that the blocking probability is:

$$
P_{R}=1-\lambda^{-1}(\mathbb{E}[I]+S)^{-1}=1-\frac{q}{[1+\lambda q S]\left[1-(1-q) e^{-\lambda q S}\right]}
$$


Note that this result can be extended to multiple job types with different values for $k$, following the arguments by Coffman Jr. et al. [113].

When we relax assumptions of this reservation model, the complexity of the calculations increases and the problems of finding (closed form) expressions for the stationary density and/or blocking probability appear to be intractable. Particularly, for the above analysis to hold, it is required that if jobs $\mathrm{A}$ and $\mathrm{B}$ request overlapping service intervals and A arrived earlier than B, then if $\mathrm{A}$ is blocked, B should also be blocked. For stochastic service times, this is not true; if the requested service interval of $B$ is contained in that of $A, B$ could be accepted while $\mathrm{A}$ is blocked by a reservation job that was accepted earlier. In the remainder of the chapter, our goal is to further develop the intuition on the consequences of advance reservation.

\subsection{The effect of advance reservation}

Advance reservation can have three possible effects on the blocking probability: none, an increase or a decrease. In this section we give three examples of the systems with independent $S$ and $R$, where $P_{0}=P_{R}$ (Theorem 1), $P_{0}<P_{R}$ (Theorem 2), and $P_{0}>P_{R}$ (Theorem 3).

Theorem 1. In any reservation system with deterministic $R$ and generally distributed $S$, it holds that $P_{0}=P_{R}$.

Proof. The sample paths of the models with and without advance reservation are identical, because the deterministic reservation time delays the start of the service by a constant $R$ for all jobs. Therefore $P_{0}=P_{R}$; see Section 5.2.2.

Theorem 2. In any reservation system with generally distributed $R$ and deterministic $S$, it holds that $P_{0} \leq P_{R}$.

Proof. The proof is based on the optimality result of the Greedy Online algorithm (GOL-algorithm) [168]. The GOL-algorithm is an online scheduling algorithm that schedules jobs at arrival to any free server. Consider a general loss system with $c$ servers. If at arrival job $k$ finds $c$ servers busy and there is a job $j$ in service with remaining service time greater than the service time of $k$, then $j$ is interrupted and lost, and $k$ is scheduled at that station. Jobs that are not served immediately, are lost. In [168] it is shown that this algorithm minimizes the number of lost jobs.

Now, suppose exactly $n$ jobs arrive in a time window $[0, T]$, with $n \in \mathbb{N}$ and $T \in \mathbb{R}$. We define a set $\mathcal{A}^{G(c)}$ containing the accepted jobs when scheduled according to the GOL-algorithm applied for capacity $c$. Moreover, the sets $\mathcal{A}^{N R(c)}$ and $\mathcal{A}^{R(c)}$ contain the accepted jobs in the system without and with reservation, respectively. It is clear that $\mathcal{A}^{G(c)}=\mathcal{A}^{N R(c)}$, because when the service requirements are deterministic, an arriving job can never have a remaining service time 
lower than that for a job already in the system. In the reservation model, the advance reservation destroys the First Come First Serve property, hence, in general, $\mathcal{A}^{R(c)} \neq \mathcal{A}^{N R(c)}$. Since $\mathcal{A}^{N R(c)}$ is optimal because the GOL-algorithm is optimal for minimizing the number of losses, we conclude that $\left|\mathcal{A}^{R(c)}\right| \leq\left|\mathcal{A}^{N R(c)}\right|$. Since $n$ and $T$ were arbitrary, this completes the proof. Note that the strict inequality $P_{0}<P_{R}$ holds if, with positive probability, we have $\left|\mathcal{A}^{R(c)}\right|<\left|\mathcal{A}^{N R(c)}\right|$.

Theorem 3. In a reservation system with one server and Poisson $(\lambda)$ arrival process, where $R$ and $S$ are independent and

$$
R=\left\{\begin{array}{ll}
0 & \text { w.p. } q, \\
T & \text { else, }
\end{array} \quad S= \begin{cases}0 & \text { w.p. } p, \\
T & \text { else, },\end{cases}\right.
$$

if $\lambda T p>1$ and $q<\frac{\lambda T p-1}{\lambda T}$, then $P_{0}>P_{R}$.

Proof. First, note that

$$
1-P_{0}=\frac{1}{1+(1-p) \lambda T}
$$

We will derive a lower bound on $1-P_{R}$ and show that this is greater than $1-P_{0}$. To this end, note that for the reservation system, jobs with $R=T$ are never blocked by jobs with $R=0$. Therefore, their acceptance probability is $(1+(1-p)(1-q) \lambda T)^{-1}$. A job with $R=0$ and $S=0$ will be accepted in the reservation model if the system is empty, which occurs with probability $(1+(1-p)(1-q) \lambda T)^{-1} \cdot(1+(1-p) q \lambda T)^{-1}$. From this, we obtain a lower bound $L$ on $1-P_{R}$ :

$$
L=\frac{1-q}{1+(1-p)(1-q) \lambda T}+\frac{p q}{[1+(1-p)(1-q) \lambda T][1+(1-p) q \lambda T]} .
$$

Now, straightforward calculations yield that for $q \in\left(0, \frac{\lambda T p-1}{\lambda T}\right)$ we have $1-P_{0}<$ $L \leq 1-P_{R}$

Theorem 3 shows that the advance reservation can increase the number of accepted jobs by favoring the shorter jobs. Indeed, the jobs with $R=T$ simply represent the $M / G / 1$ queue, and in the time when this queue is vacant, all jobs with $R=S=0$ are accepted, while some jobs with $R=0, S=T$ are rejected.

In the next section we numerically investigate the impact of a range of model parameters on the blocking probability of the reservation system via discrete event simulation.

\subsection{Numerical results}

The results of this section are based on 15 runs with each $10 \mathrm{M}$ arriving jobs, which is sufficient to obtain $99 \%$ confidence intervals with a relative precision 
of less than $1 \%$. Below, the results are given without specifying the confidence intervals.

We will first investigate the influence of the coefficient of variation of the service time. To this end, for $c=1$ and $\lambda=6$ we now consider a discrete service time distribution with $S=\frac{1}{p} \mathbb{E}(S)-0.1$ with probability $p$ and $S=\frac{0.1 p}{1-p}$ with probability $1-p$. Let $R$ be exponential with rate two and $\mathbb{E}(S)=1 / 3$ or $\mathbb{E}(S)=1 / 7.5$. Figure 5.3 displays the blocking probabilities and $c_{v}(S)$ for varying p. For $c_{v}(S)=0$, the blocking probability in the reservation system is maxi-

Figure 5.3 Blocking probability (left axis) for varying $c_{v}(S)$ (right axis), for $\mathbb{E}[S]=$ $1 / 3$ (left) and $\mathbb{E}[S]=1 / 7.5$ (right).

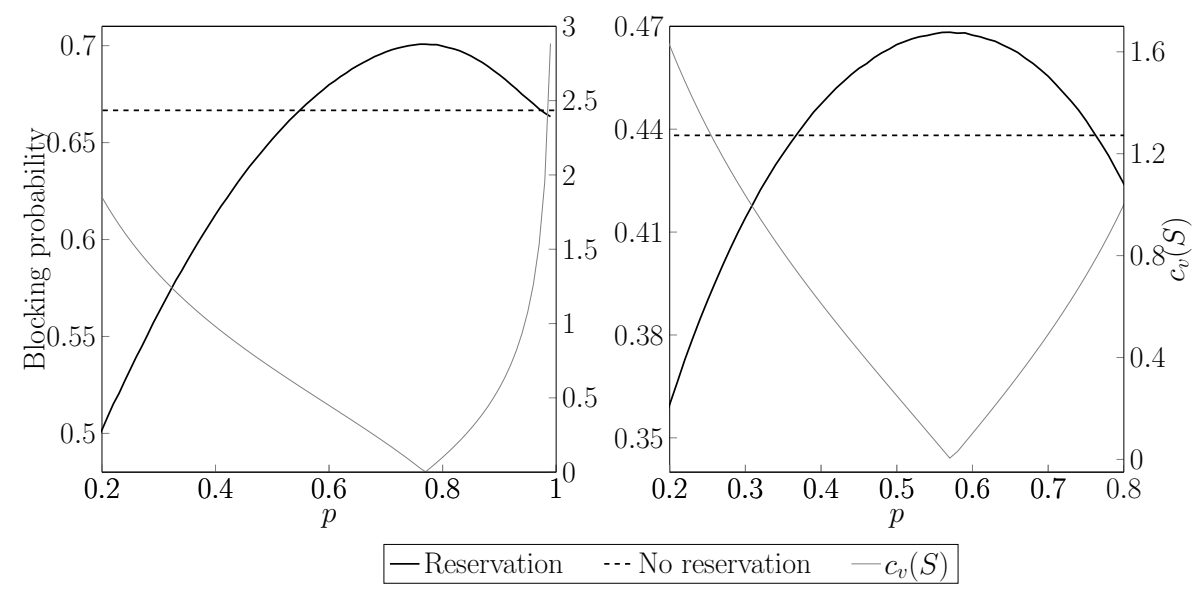

mal, and blocking probabilities decrease with increasing $c_{v}(S)$. Furthermore, for small $c_{v}(S)$, blocking probabilities with reservation exceed those without reservation, while for higher $c_{v}(S)$ blocking probabilities with reservation tend to be lower than the corresponding blocking probabilities without reservation. This illustrates that short jobs are accepted in favor of long jobs, because in this numerical example high $c_{v}(S)$ implies relatively many arriving jobs are short.

This is illustrated again in Figure 5.4. Here for $c=1, \lambda=6.1, \mathbb{E}[R]=1 / 2$ and $\mathbb{E}[S]=1 / 3$, both the blocking probability (left) and logarithm of the total number of blocked jobs in the simulation run (right) for exponential notice and service times are displayed as a function of the attained service time. Clearly, without advance reservation the lengths of the jobs and the number of accepted jobs are independent. However, with advance reservation, the blocking probability is very low for small $S$, and higher than in the $M / M / 1$ system for larger values of $S$. Since the probability of a large service time is low, there is little difference in the absolute number of blocked long jobs in both systems. 
Figure 5.4 The blocking probability and log number of blocked jobs as function of the attained service time for $R, S \sim$ Exp.

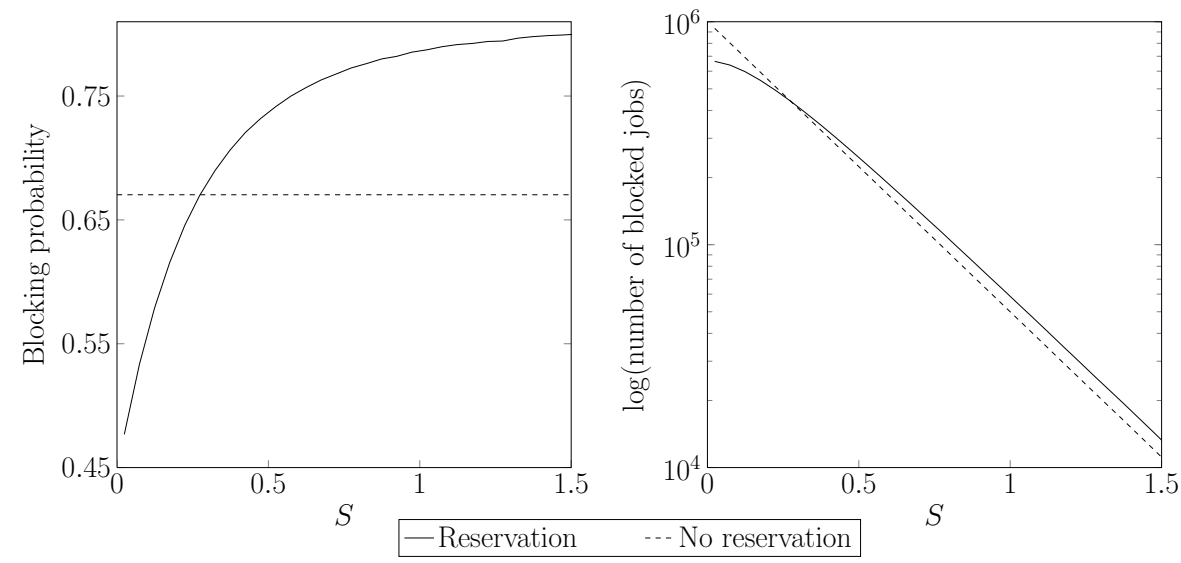

\subsection{Conclusion}

In this chapter we have identified classes of loss queues where the advance reservation results in increased or decreased blocking probabilities. Our numerical results indicate that blocking probabilities decrease with increasing variability of the service times. Reduction of blocking probabilities is achieved due to the acceptance of more shorter jobs at the cost of larger jobs.

In these lines, an interesting open question is, for example, the asymptotic behavior of the system when one of the parameters becomes much larger than others. For instance, with $\lambda$ fixed and $\mathbb{E}[S] \rightarrow \infty$ the notice time becomes irrelevant and the blocking probability with and without reservation will be equal. However, with fixed $\mathbb{E}[S]$ and $\lambda \rightarrow \infty$, assuming, for example, exponential service and notice times, the idle times in the system with reservation can be filled out by shorter jobs. Hence, it is interesting to find the limiting relation between $P_{R}$ and $P_{0}$.

In general, the trade-off between reservation and blocking probabilities is intricate. Designing efficient and equitable reservation systems continues to be a challenging task. 



\section{Part III}

\section{Patient appointment schedules}

\section{Chapter 6}

N.M. van de Vrugt, R.J. Boucherie, T.J. Smilde, M. de Jong, and M. Bessems. Rapid diagnoses at the Breast center of Jeroen Bosch Hospital: a case study invoking queueing theory and discrete event simulation. Submitted.

\section{Chapter 7}

N.M. van de Vrugt, P. Matel, R.J. Boucherie, P. van Engelen, T. Beukman, and J. de Laat. Verbeterde afsprakenplanning voor patiënt en gipsverbandmeester. [In Dutch] In dit Verband, 26(1): 6-7, 2016. 



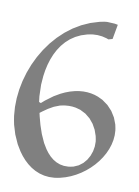

\section{Rapid diagnoses at the breast center}

\subsection{Introduction}

Breast cancer is the most prevalent cancer type for Dutch women, with incidence rate doubling over the last two decades [371]. At an early stage, most breast cancer cases can be treated successfully. When suspected tissue is discovered in a patient's breast, swiftly available diagnostic test results are essential for medical and even more for psychological reasons: waiting for a diagnose is demanding for the patient and her family and friends.

In the Netherlands, patients with suspected breast cancer are referred by their general practitioner (GP) or the Dutch national screening program. Upon referral, patients may choose to go to one of the (academic) hospitals or specialized breast cancer clinics. The JBH breast center is an outpatient clinic in which 10 specialties are represented or on call during office hours. At the center a patient undergoes diagnostic tests, receives their outcome, and if necessary receives treatment and follow-up care. This case study reports on improvements in the diagnosis phase of the process, as depicted in Figure 6.1.

Dutch breast cancer centers have recently increased their efforts to reduce

Figure 6.1 Process description for a patient at the breast center, 'NP' is a consult with the nurse practitioner.

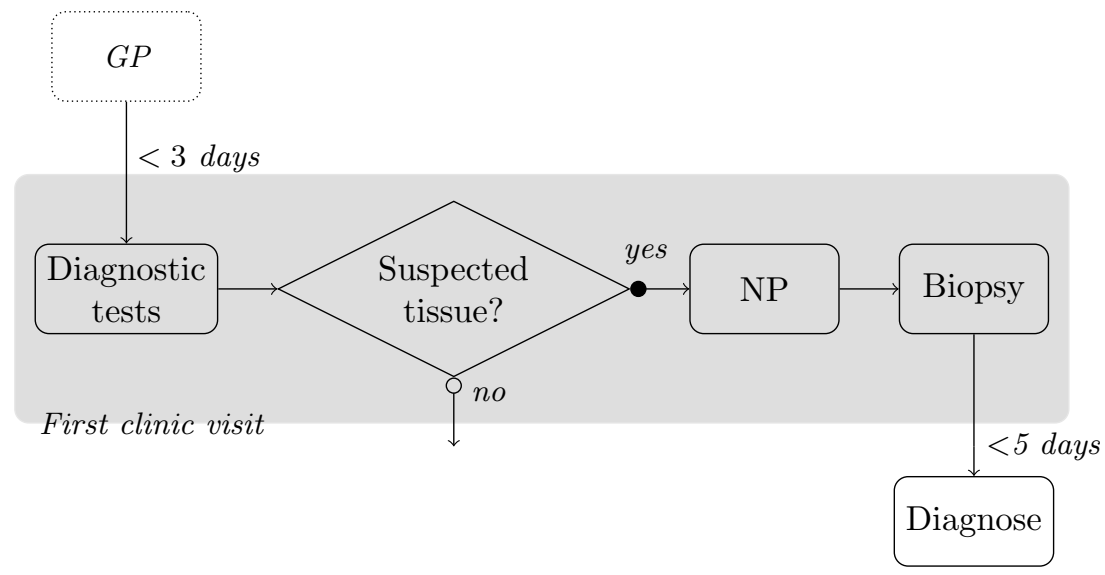


the time from patients' referrals to their first appointments at the clinic (access time). In accordance with the recent national standards, the JBH aims for $90 \%$ of patients to receive an appointment within three working days. National guidelines require that the access time is at most five working days for $90 \%$ of the patients [368]. If a patient's preliminary diagnosis is negative (no cancer), she immediately receives the diagnosis and goes home. Otherwise, the patient consults a nurse practitioner and a biopsy is taken. All patients that got a biopsy must be discussed in a multidisciplinary meeting (MDM) after the laboratory results are available, and the patient receives an appointment at the center at a later day to discuss the test results. National guidelines require that $90 \%$ of the patients that got a biopsy receive their diagnosis within a week [368].

Accurately measuring the access time is in most hospitals impossible without additional (manual) data collection, as hospitals often only register the appointment time and not the time the appointment was requested. At the JBH, the data system of the radiology department does register the time at which an appointment is made. However, the data system only discriminates between new and returning patients accurately for patients originating from the national screening program (hereafter: screening patients). Before the interventions reported in this case study only $54 \%$ of the new screening patients were seen within three working days, which is lower than the $90 \%$ the JBH aims for. The time from biopsy to the appointment at the center was registered for all new patients; before the interventions $89 \%$ of the patients got their diagnosis within five working days, which is close to the $90 \%$ target of the $\mathrm{JBH}$.

For designing and implementing interventions, the JBH formed a steering group consisting of all stakeholders in the diagnostic part of the breast cancer care pathway: a radiologist, an oncologist, a surgeon, the capacity coordinator (who managed the entire breast cancer care pathway), and two OR-specialists that were part-time at the JBH. One of the interventions focused on the most effective time for an extra MDM such that the requirements for the time to diagnosis could be met. Based on the results of a discrete time queueing model, a new patient schedule was implemented at the center. After these interventions $84 \%$ of new screening patients were seen within three working days (was $54 \%$ before the intervention), and $92 \%$ of the diagnoses of all new patients were delivered in time. Currently, the JBH is investigating other possibilities to further reduce the access time. As a follow up on the reported interventions, JHB aims to minimize the accumulated time a patient waits on the day of their radiological examinations (waiting time). In this chapter we propose a promising new patient schedule based on the results of a discrete event simulation (DES).

In this case study we present a business re-engineering approach based on OR that is carried out in a hospital and has resulted in considerable improvements of the performance. This is a clear advertisement for the power of OR for process improvements and for real implementation in practice and a guideline for how to achieve implementation of OR in practice. The remainder of this chapter is organized as follows. We first review the related literature in Section 6.2. In 
Sections 6.3 and 6.4 the interventions for the access time and time to diagnosis are discussed, respectively. Section 6.5 considers optimizing the waiting time, and the last section contains our conclusions.

\subsection{Related literature}

At the breast center patients require a series of appointments with multiple resources, of which some are unscheduled before the patient arrives at the center. There are multiple patient types and each type may have its own access time norm. In this section we first discuss literature that is related to the tactical appointment scheduling problems (see for example [242]) of minimizing access and waiting times. Thereafter, we highlight literature that encompasses implementation results of $\mathrm{OR}$ models in practice.

Literature on minimizing access time for patients that require multiple appointments is sparse, as already stated in Chapter 4. In a general hospital setting, Hulshof et al. [243] optimize the number of elective patients of each type that can be admitted to the hospital on a certain day invoking a mixed integer program. Objectives are access time norm compliance and achieving the hospital's predefined targets on the number of patients to treat. Upon arrival patients appear to require a certain (deterministic) care pathway, and therefore a patient's admission can be viewed as an appointment. Matta and Patterson [350] use DES to evaluate many possible interventions at an oncological outpatient clinic, of which two interventions consider rules for scheduling patients. This work mainly focuses on patients requiring a single appointment, in accordance with the general outpatient clinic literature $[43,83]$. A different related field of literature is on patients who receive radiotherapy treatment. These patients also require multiple appointments at multiple resources, see for example [47, 392], but these are all scheduled in advance. Additionally, there are many papers on tactical scheduling of a single appointment on a single resource for multiple patient types with the objective to minimize access times, see for example [23, 128, 233, 270, 298, 301, 428].

The literature on minimizing patients' waiting time while minimizing the doctors' idle and overtime is also sparse for systems in which patients require multiple appointments on multiple resources. In a clinic where patients require multiple appointments, Wu et al. [522] use DES to determine the best appointment scheduling rules with respect to these objectives. Griffin et al. [200] simulate different scenarios for an obstetric clinic, in which each patient is randomly assigned to one of the possible care pathways, but in obstetric clinics most patients are urgent and not scheduled in advance. For a family practice where patients require an appointment with both a nurse and doctor, Oh et al. [380] invoke a stochastic integer program combined with heuristics to optimize the schedule. Stochasticity is present in the service time durations, but not the care pathways of the patients. The setting studied in [522] is most related to our case study setting since part of the care pathway is stochastic. There are many papers on scheduling a single appointment on one resource that balance waiting, idle, and 
overtime, see for example [139, 270, 272, 409].

For additional outpatient clinic literature for both the tactical and operational level, the reader is referred to the reviews [43, 83]. For general appointment scheduling literature in healthcare see the reviews [212, 213, 242, 385].

Many recent reviews, including the one in Chapter 2, concluded that the literature on OR in healthcare that reports on (the results of) actual implementation of the findings is sparse [65, 66, 83, 212, 302, 336, 427]. For different types of simulation applications specifically, van Lent et al. [302] conclude that only three papers (partially) report on the effects of the implementation in real life. Mahdavi et al. [336] have found 10 papers reporting on implementation results, of which two are related to this chapter as they focus on an outpatient clinic $[13,550]$. Both papers use the queueing network analyzer (QNA) of Whitt [516] to evaluate different alterations to the process at the clinic, but only consider unscheduled arrivals. Matta and Patterson [350] also report on implementation of some of their recommendations.

Concluding, the literature on scheduling multiple appointments on multiple resources is sparse, just like the papers mentioning implementation of their results in practice. Our case study reports on an OR-model developed for the multi-disciplinary breast center of the JBH, it's implementation results, and the lessons learned from the cooperation between OR-specialists and healthcare practitioners.

\subsection{Access time}

At the breast center of the JBH, new patients originate from two sources: the national screening program and the GP. In the Netherlands all women aged 50-75 are invited every two years by a screening organization for preventive diagnostic tests [369]. Patients referred by their GP usually have palpable abnormalities or a heredity risk. In accordance with national guidelines [368], new patients aged $30+$ are scheduled for a mammography and an ultrasound, and younger patients only for an ultrasound. Younger patients can also be scheduled for an MRI examination, but as the MRI center of the JBH currently meets all its access time requirements, these patients are not considered in this project. We distinguish three types of new patients: patients referred by the screening program (always age $\geq 50$ ), young patients (age <30) and 'regular' patients referred by their GP. The JBH aims for the access time to be less than three working days for $90 \%$ of the patients, but according to national guidelines it should at least be within five working days for $90 \%$ of the patients [368].

\subsubsection{Before intervention}

The available JBH data only allows to accurately distinguish new screening patients as new patients. Together with the radiology department, we determined an inclusion criterion to estimate the fraction of new patients for the other patient 
types. We determined the rate at which each new patient type arrives for each day, see Figure 6.2, where the upper and lower edge of each box represent the $75 \%$ and $25 \%$ percentiles, respectively, and the whiskers extend to the minimum and maximum number of arrivals per day. We define the capacity of the radiology

Figure 6.2 Boxplot of the arrivals per type for each day of the week (Jan. '13 - July '14).

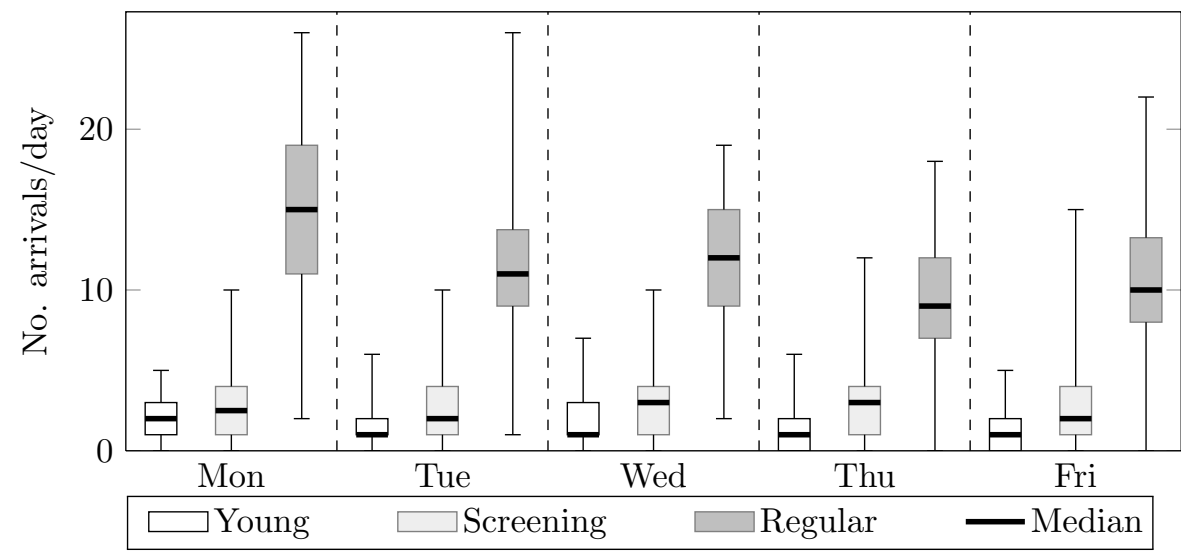

department by the number of appointment slots available for each patient type. This capacity is the same for each day and equals: 4 young, 5 screening and 18 regular patients. From Figure 6.2 it is clear that the demand is not constant over the weekdays. On average there seems to be enough capacity to cope with the current demand of new patients.

At the JBH only for $34 \%$ of the young patients, $31 \%$ of the regular patients, and $54 \%$ of the screening patients the access time was less than three working days. Only for the screening patients the national guideline was met, while $33 \%$ of the young patients and $28 \%$ of the regular patients had access times longer than five working days. As the inclusion criterion provided an estimate of the fraction of new young and regular patients, the criterion may result in an overestimation of the access times for these patients.

\subsubsection{Intervention}

Each day a random number of patients arrives at the clinic, and a certain number of patients receives an appointment. All patients receive the first appointment available for their type, but are generally not scheduled for the same day as their appointment request. Patients may not be deferred to other clinics, so all arriving patients must receive an appointment. We are interested in determining the daily capacity such that the clinic complies with the access time norms.

We developed a discrete time queueing model similar to the one presented in [281] to evaluate the access time distribution of new patients, measured in number of days. A queueing model is preferred over optimization or simulation models since queueing models reveal the access time distribution. 
The state of the queueing model is the size of the backlog of a specific patient type, so the number of patients that did not have their tests yet. Every day a number of patients (at most equal to the capacity for this type on this day) is removed from the backlog, and a random number of new patients is added to the backlog. We incorporate that patients cannot be scheduled for their first appointment on the day of their arrival. We obtain a discrete, empirical probability distribution for the number of arrivals for each weekday from the hospital data, and use a cyclic schedule with a period of five days. All details of the model can be found in Appendix 6.7.

By means of our queueing model we derived the minimal capacity required to meet the JBH guidelines, see Table 6.1. The performance of the schedule

Table 6.1 Minimal capacity required at the JBZ.

\begin{tabular}{lccc}
\hline & Young & Screening & Other \\
\hline Mon & 3 & 5 & 15 \\
Tue & 3 & 5 & 14 \\
Wed & 3 & 5 & 16 \\
Thu & 2 & 5 & 16 \\
Fri & 3 & 6 & 14 \\
\hline
\end{tabular}

with minimal capacity and the current schedule can be found in Figure 6.3. Note that the minimal capacity for young and regular patients is less than the current capacity, which seems to contradict the fact that the guidelines were not met with the current capacity. After we presented the results, the radiology department reconsidered the available capacity, and became aware that the realized capacity for regular patients was usually less than 18 slots (as scheduled) and often less than 15 (average minimal capacity); each day several slots were closed because one or two patients needed to be prepared for surgery and one radiologist had to prepare the MDMs.

Figure 6.3 Access time distribution obtained from the queueing model.

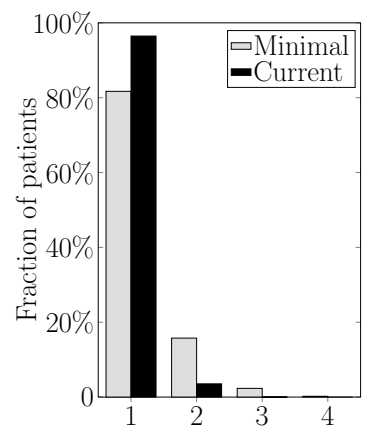

Access time (days) - Young

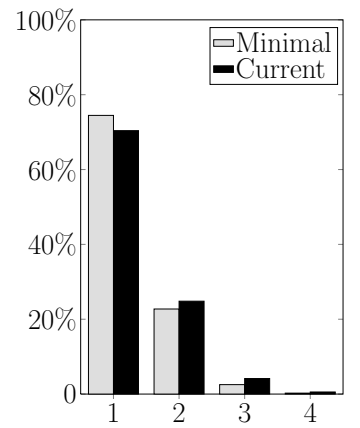

Access time (days) - Screening

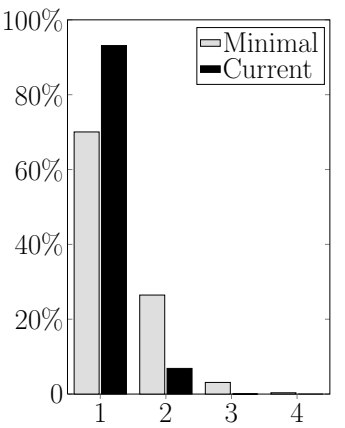

Access time (days) - Regular

Discussions of the results with the radiology staff resulted in two additional 
interventions. First, data analysis indicated that the time to take a mammography and ultrasound for returning patients was shorter than the appointment length. The radiology assistants confirmed that returning patients require less examination time. Therefore, in our intervention all returning patients are scheduled for 15 minute appointments instead of 20 minutes for both mammography and ultrasound tests, thereby increasing the number of daily appointment slots by three. Second, the radiology assistants felt that almost all screening patients required a tomography, which was confirmed by the data. Combining a mammography with a tomography for the new screening patients reduces the processing time of the latter and saves the time that patients (un)dress and change rooms.

The resulting intervention was: to assign slots for the preparations, and schedule on average 1.6 slots per day for young patients, 5 slots per day for screening patients, 15.4 slots per day for regular patients, and 2 flexible slots each day. Additionally, young patients were preferably scheduled for a 3D-ultrasound to both improve diagnostics and alleviate the utilization of the regular ultrasound rooms. By these interventions, the total capacity for new patients theoretically decreased from 27 to 24 slots per day, but in reality it increased since in the process after the intervention it is no longer allowed to close slots that were reserved for new patients. The interventions were implemented in July 2014.

\subsubsection{Result}

Comparing data from January 2013 to October 2014, we conclude that for all patient types the access time decreased, see Figure 6.4. From the data it appeared that especially young patients, who require only an ultrasound, were often scheduled for same-day appointments after the intervention. For the screening

Figure 6.4 Access time distribution before (Jan. '13 - July '14) and after (July '14 - Oct. '14) interventions.
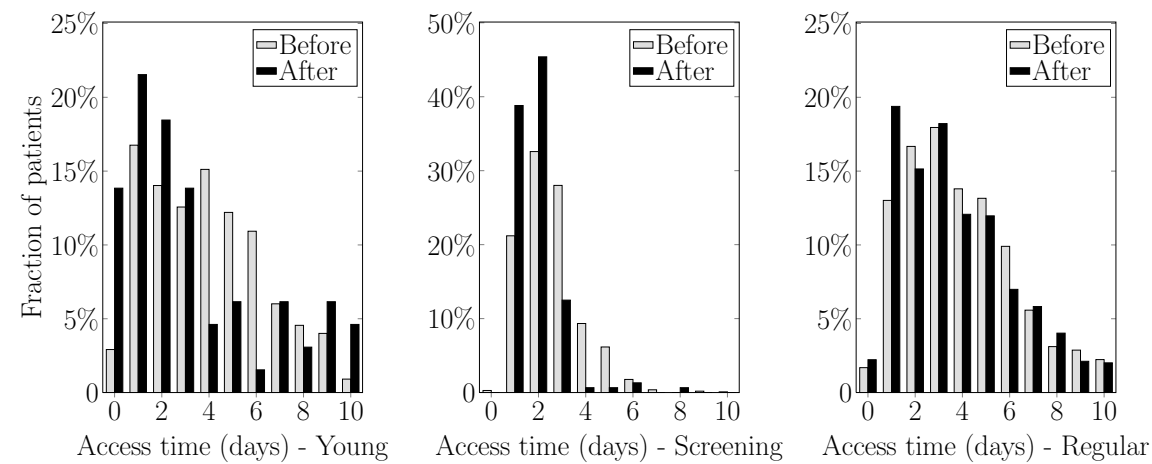

patients the access time improved the most. However, still for none of the patient types the target of $90 \%$ within three days is met. Recall that for young and regular patients these results underestimate the real performance, which is confirmed by the fact that patient surveys indicate that $85 \%$ of the patients have an access 
time of five working days or less. The steering group is currently aiming for more improvements, which will be discussed in Section 6.6.

\subsection{Time to diagnosis}

For the time to diagnosis, two groups of patients may be distinguished. Patients that do not require a biopsy receive their diagnosis immediately after their last radiologic examination. If a patient does require a biopsy, the extracted tissue is processed by the laboratory on the same day. This process, immunostaining, takes two to five days, where five days rarely occurs. In the Netherlands, the results of a biopsy have to be discussed in an MDM before they can be communicated to the patient. Patients receive a follow-up appointment at the breast center to discuss their test results. The time between the biopsy and the followup appointment should be less than or equal to five working days for $90 \%$ of the patients [368].

\subsubsection{Before intervention}

The time between the biopsy and the follow-up appointment at the center was influenced both by the processing time at the laboratory and the frequency of the MDMs. The processing time at the laboratory depended on the time the biopsy was taken: if it was taken before noon, the results were available at the end of the next day, and otherwise at the end of the second day. MDMs were scheduled on Wednesday and Friday afternoon each week. The lab results that were ready on Wednesdays and Fridays could not be discussed in the MDM on the same day, since the results were available too late to prepare for the MDM. Before the interventions, $89 \%$ of the diagnoses were given within five working days.

A process description of the steps from biopsy to the availability of the diagnosis revealed a structural problem at the JBZ. For each morning and afternoon of each day, we determined the earliest time that each process step could be finished if a biopsy was taken on that day, see Table 6.2. From this table it appears that biopsies taken on Thursday morning and in the afternoon of Monday, Wednesday and Thursday, could never be communicated to the patients within five working days because of the way the MDMs were scheduled. Moreover, the number of patients who finished their biopsy before noon was limited; due to the lengths of the tests and the availability of the radiologists, only the patients with appointments before 9:20 could have their biopsy finished before noon.

\subsubsection{Interventions}

We proposed three possible interventions: an extra MDM, shorten the processing times at the laboratory, and only schedule returning patients (and no new patients) on Thursdays. The latter option was very attractive to the radiology department because fewer additional tests were required on such days, increasing the number of patients that could be seen on one day. The department 
Table 6.2 Process from biopsy to diagnosis before the interventions.

\begin{tabular}{llccc}
\hline Biopsy taken & Lab finished & MDM & Follow-up app. \\
\hline \multirow{2}{*}{ Monday } & Morning & Tue & Wed & Thu \\
& Afternoon & Wed & Fri & Mon \\
\hline \multirow{2}{*}{ Tuesday } & Morning & Wed & Fri & Mon \\
& Afternoon & Thu & Fri & Mon \\
\hline \multirow{2}{*}{ Wednesday } & Morning & Thu & Fri & Mon \\
& Afternoon & Fri & Wed & Thu \\
\hline \multirow{2}{*}{ Thursday } & Morning & Fri & Wed & Thu \\
& Afternoon & Mon & Wed & Thu \\
\hline \multirow{2}{*}{ Friday } & Morning & Mon & Wed & Thu \\
& Afternoon & Mon & Wed & Thu \\
\hline
\end{tabular}

organized two trial days on which only returning patients were scheduled. From these days, it appeared that the follow-up appointments with the surgeons could not be scheduled with this number of patients seen on one day. Therefore, this solution was not taken into account.

Both the first and second solution were implemented in April 2014 at the JBH; each Monday at lunch break an additional short MDM was scheduled, and the laboratory bought faster machines so the result of all (both morning and afternoon) biopsies are available the next day. This resulted in the 'earliest finish times' of the process reported in Table 6.3. Note that, since the MDM on

Table 6.3 Process description with additional MDM and faster laboratory.

\begin{tabular}{lccc}
\hline Biopsy taken on & Lab finished & MDM & Follow-up app. \\
\hline Monday & Tue & Wed & Thu \\
Tuesday & Wed & Fri & Mon \\
Wednesday & Thu & Fri & Mon \\
Thursday & Fri & Mon* & Mon \\
Friday & Mon & Wed & Thu \\
\hline
\end{tabular}

Monday (indicated with *) is in the lunch break, the diagnoses of the discussed patients can be communicated on the same day. From Table 6.3 it is clear that all diagnoses could in principle be communicated to the patients within five working days.

\subsubsection{Results}

From data from April 2014 until October 2014 it appeared that after the interventions $92 \%$ of the patients received their diagnosis within five working days. 
Although this is only a minor improvement, it resulted in meeting the guideline. In practise it will not be possible to achieve $100 \%$ within five working days, because sometimes an additional test needs to be performed to ascertain the diagnosis.

\subsection{Waiting time}

Although arrivals to the center are scheduled, the patients' pathways through the center are not deterministic. All young patients receive an appointment for an ultrasound, whereas screening, regular and returning patients receive appointments for both a mammography and an ultrasound. After the mammography is completed, it may appear that the ultrasound is not necessary any more, or that the patient requires additional tests (e.g., 3D-ultrasound, tomography or biopsy). All screening patients and patients that require a biopsy (which are not necessarily disjunct sets) visit a nurse practitioner to receive additional information about their diagnosis and/or the biopsy.

Almost all additional tests are performed on the same day, only MRI scans and stereotactic (mammography based) biopsies are scheduled for later days. The tomographies and biopsies are performed on the same resources as the mammographies and ultrasounds, respectively. Since both resources are fully booked in advance, this induces waiting time.

The waiting time for additional tests is distressing patients, since they know that the results of the first tests indicated that additional tests were necessary, implying that they might have breast cancer. Before this project started, the radiology assistants were always assigned to one patient throughout her stay at the radiology department. Therefore, when their patient was waiting, they were waiting too. This varying pressure of work resulted in reduced work satisfaction of the staff. There are no (national) guidelines for patients' waiting times, but the JBH aims to minimize waiting time while maintaining the same working hours.

\subsubsection{Approach}

For most hospitals it is hard to measure the waiting time from data as the start and end time of an appointment is usually not registered, and the JBH is no exception. Instead of taking measurements manually, we decided to build a proof of concept DES model to advise the radiology department on possible improvements. Input for the DES is a patient appointment schedule, a radiologists schedule, and for each patient type the probability of requiring a certain series of tests. For more details on the DES, see Appendix 6.8.

From the literature sequencing models and stochastic integer programming models appear promising approaches for optimizing the patient schedule. However, with these methods it is not clear how to incorporate that patients have to return to a resource with a different service time. Additionally, we expect the state space to explode since we schedule 40 patients, each following one of the 
11 possible pathways with a probability depending on her type. A simulation model can incorporate all necessary details of the patients' pathways and can be used to quickly evaluate many schedules. Therefore, we constructed several possible patient schedules manually and used the DES to fine-tune the schedule iteratively.

\subsubsection{Simulation results}

We evaluated both the patient appointment schedule used before the project started and after the interventions were implemented. From the DES it appears that the variability of the process is large; even with 520 simulated days the relative precision is not below $20 \%$, and for young patients even around $70 \%$. This confirms the feeling of the staff about strongly varying workloads. Invoking common random numbers [294], the results for the different schedules may be compared, and the variability of the differences between scenarios is reduced.

In Figure 6.5 we depicted boxplots of the waiting time per patient accumulated for all tests, for all simulated working days for the schedule before July 2014 (Before), after July 2014 (Current), and the improved schedule. It appears that the interventions of July significantly decreased the waiting time, especially for regular patients.

Figure 6.5 Simulation results for the accumulated waiting time per patient.

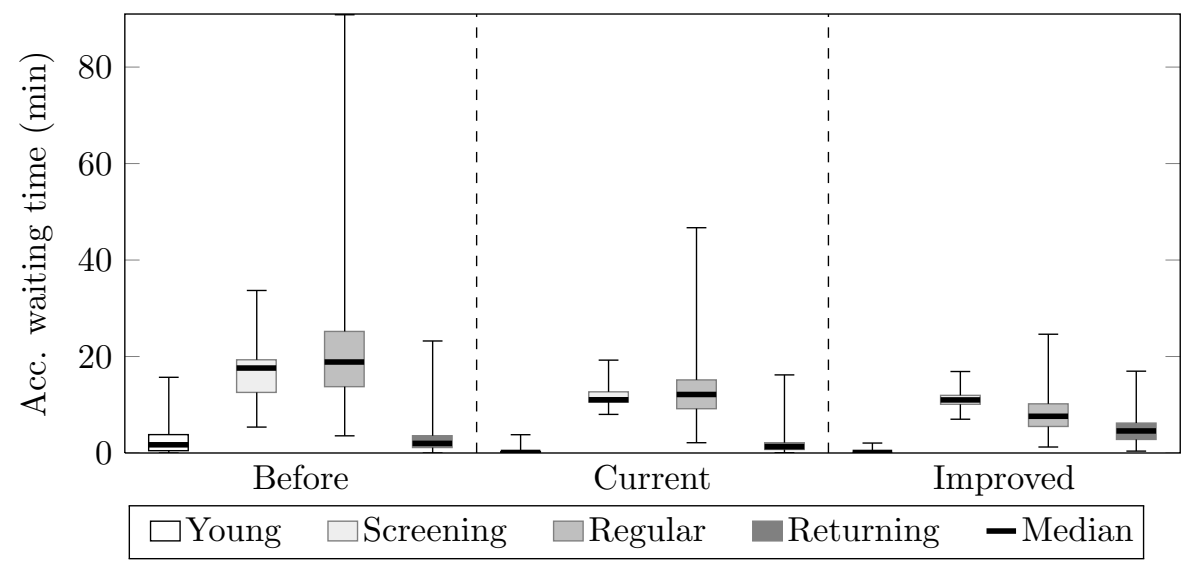

The improvements we made to the schedule were scheduling five minutes idle time between appointments for screening patients (to allow for the radiologist to check the test results), add two slots for (unscheduled) biopsies, and distribute the returning patient slots evenly over the entire day (opposed to only new patients in the morning and returning patients in the afternoon). Additionally, we distributed the empty slots already present in the schedule more evenly over the day to be able to provide additional tests. The best distribution of this idle time was obtained through several iterations of simulation and alterations to the schedule. This schedule reduces the waiting time for all patient types, except the 
average waiting time of returning patients (compared to the schedule currently used), see Figure 6.5.

Figure 6.6 Simulation results for the treatment rooms (f.l.t.r): two mammography rooms, two ultrasound rooms, a 3D ultrasound room and the nurse practitioner.

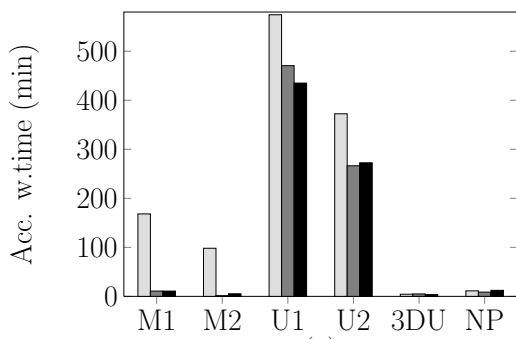

(a)

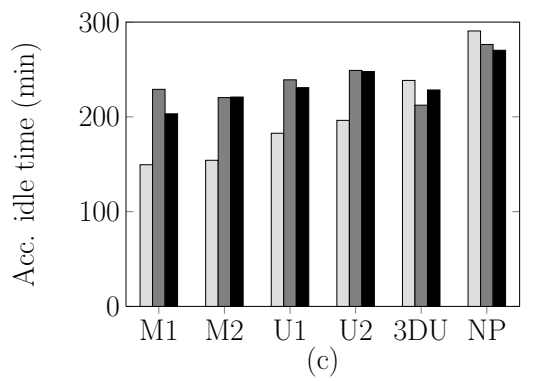

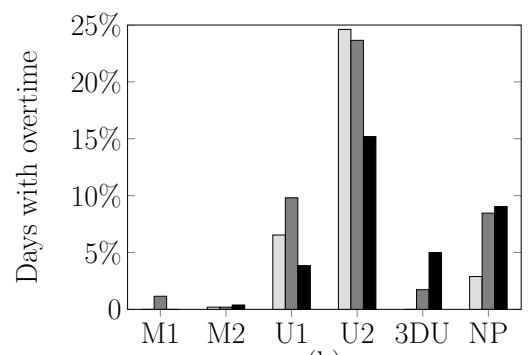

(b)

Figure 6.6 (b) shows that the current schedule results in many days with overtime for both the ultrasound rooms and the nurse practitioner, and the improved schedule induces fewer days with overtime. Both mammography rooms and the 3D-ultrasound room finished within office hours on most of the days. Figure 6.6 (a) displays the accumulated waiting time of all patients scheduled on a room, and Figure 6.6 (c) the accumulated idle time (including breaks) before the last patient of the day leaves. For both mammography rooms and the 3D ultrasound room, patients do not wait long for their tests in the current and improved schedule. The percentage of overtime days for ultrasound room 1 is acceptable, but $9 \%$ for the nurse practitioner and $15 \%$ for ultrasound room 2 are too high. Attempts to decrease the number of overtime days resulted in unacceptable higher waiting time for patients. Therefore, the breast center is currently investigating other possibilities to reduce the waiting time, for example by altering the order of the current process steps such as letting the patient go first to the nurse practitioner in order to take the biopsy directly after the ultrasound. This would reduce the time required for a biopsy significantly.

Regarding the robustness of the results, we performed several tests with the DES. When we assume that the service time durations are not a constant $S$ but uniformly distributed on the interval $[0.9 S, 1.1 S]$, the results of the DES are similar for the improved schedule, but with higher variation between simulated days and some exceptional days with high waiting times. We also investigated 
the effect of non-punctual patients, by assuming that patients arrive a random time from their appointment time. We used a Normal distribution with $\sigma=10$ minutes and three different means: $-10,0$, and 10 minutes. It appears that when patients arrive early, the performance of the schedule improves slightly. For patients that arrive in time on average (mean 0), the performance of the schedule is similar to the schedule in the original DES. Only when patients on average arrive 10 minutes late, the number of days working in overtime increases significantly, but there is a slight decrease in the waiting time for patients as well. For the patients the performance is still quite similar to the performance in the original DES, but there are some exceptional days in the DES with relatively high accumulated waiting times. See Appendix 6.8 for the exact results of the robustness analysis.

The assumption of punctual patients is not entirely realistic, but the JBH staff confirms that most patients arrive early for their appointments. Both the data and the JBH staff indicate that the assumption of deterministic service times is close to reality, but the tested perturbation is relatively low. Therefore, there probably will be days with exceptional high waiting times in reality.

\subsubsection{Intervention}

Although the proposed appointment schedule is not yet implemented, the process at the center has improved during this project. At our suggestion, the laboratory assistants worked in one room the entire day, instead of following patients throughout their stay at the radiology department. The assistants were worried that this intervention would decrease patient-friendliness, but after two trail days all were convinced that this intervention reduced the assistant's waiting time significantly and did not affect the patient-friendliness too much.

\subsection{Conclusion}

This chapter reports on a case study with a business re-engineering approach based on OR methods that is carried out in a hospital and has resulted in considerable improvements of the performance. This case study was performed through a successful cooperation between JBH healthcare practitioners and ORspecialists. In this project, the steering group and stakeholders secured the proposed OR-approach, and were the key to the successful implementation of the interventions. The OR approach provided a rigorous analysis of the performance of the system, which made the stakeholders aware of additional hidden capacity problems. Our interventions have improved the use of the available capacity, by both making additional capacity available via adapting the length of appointment slots, and rearranging the patient appointment schedule. As a consequence, at the breast center of the JBH both the access time and time to diagnosis improved evidently. In addition, we obtained promising results for reducing patients' waiting times. The cooperation had additional positive side-effects that further improved 
the process at the center. This case study is a clear advertisement for the power of OR methods for process improvements and for real implementation in practice and a guideline for how to achieve implementation of OR in practice.

It appeared from this project that the resources at the center are not sufficient to both meet the access time guidelines and work within office hours for a high percentage of days. In accordance with national trends, the steering group is currently investigating the possibility of offering trajectories which provide a diagnosis withing 24 hours, requiring daily MDMs and possibly larger opening hours. The DES built in this project will be extended to investigate the requirements for offering the 24h-diagnostics. Future research will also focus on the care pathway after diagnosis, i.e. the operating theater and radiation therapy schedules. The JBH, like most other hospitals, has a continuous urge to improve the processes for their patients (and thus actually improving efficiency). Therefore, the cooperation between healthcare practitioners and OR-specialists will be continued in future projects.

\subsection{Appendix: Discrete time queueing model}

We provide the formulas relating the daily capacity to the access time distribution through the stationary distribution of $B_{d}$, the backlog of a specific patient type on day $d$. To enhance readability all patient type indices are omitted. These results are similar to the ones presented in [281].

We assume arrivals for day $d$ occur according to a discrete distribution $A_{d}$, with $P\left(A_{d}=i\right)$ obtained from hospital data. Each day $d$ a maximum number of patients $c_{d}$ can be seen. The schedule is cyclic, so $d \in\{0,1, \ldots, D-1\}$ and days are counted modulo $D$. At the beginning of each day $d$, we have a backlog of patients $B_{d}$. We assume that patients cannot be seen on the same day, for example because they need to make arrangements at their work or some time is required for the GP to send in the results of the screening program. For this discrete time queueing model it holds

$$
B_{d}=\left(B_{d-1}-c_{d}\right)^{+}+A_{d} .
$$

The transition probabilities for this model are given by:

$$
P\left(B_{d}=q^{\prime} \mid B_{d-1}=q\right)=P\left(A_{d}=q^{\prime}-\left(q-c_{d}\right)^{+}\right) .
$$

We fill transition probability matrix $\mathbf{P}$ with the transition probabilities and the distribution of $A^{d}$, and obtain the steady state distribution $\pi=\left[\pi^{0} \pi^{1} \cdots \pi^{D-1}\right]$ solving $\pi \mathbf{P}=\pi$ and $\sum \pi=1$. Let $\pi_{d i}$ the stationary probability of having $i$ patients in the backlog on day $d$. Define $c_{d}(y):=\sum_{i=1}^{y} c_{d+i}$ the sum of the capacity from day $d+1$ until day $y$, and let $A T_{d}$ denote the access time of a patient arriving on day $d$. Then $P\left(A T_{d}>y \mid B_{d}=q\right)=1$ if $c_{d}(y) \leq q$, and for $c_{d}(y)>q$ it holds:

$$
P\left(A T_{d}>y \mid B_{d}=q\right)=\frac{\sum_{j=c_{d}(y)+1}^{\infty}\left(j-c_{d}(y)\right) P\left(A_{d}=j\right)}{\mathbb{E}\left[A_{d}\right]} .
$$


Therefore,

$$
P\left(A T_{d}>y\right)=\sum_{i=0}^{\infty} P\left(A T_{d}>y \mid B_{d}=i\right) \cdot \pi_{d i} .
$$

Moreover, the fraction of arriving jobs for which the access time does not exceed $y, F(y)$, is given by:

$$
F(y)=\sum_{d=0}^{D-1}\left\{1-P\left(A T_{d}>y\right)\right\} \frac{\mathbb{E}\left[A_{d}\right]}{\sum_{q=0}^{D-1} \mathbb{E}\left[A_{q}\right]} .
$$

Note that patient types are independent, so for each type we may evaluate the access time distribution separately.

\subsection{Appendix: Discrete event simulation model}

We built a DES that takes into account all model assumptions, with input a schedule with patient types. For each patient type, probabilities that a patient of this type has to take a certain combination of tests are obtained from hospital data, see Table 6.4, in which we used the following abbreviations: MG for mammography; CR indicates that the radiologist should check the test results (this time is also used for cleaning the machines and for patients to get (un)dressed); US for ultrasound; NP for nurse practitioner; BI for biopsy; 3D for 3D ultrasound; and TG for tomography.

\begin{tabular}{|c|c|c|c|c|c|c|c|c|}
\hline \multicolumn{5}{|c|}{ Care pathway } & \multirow[t]{2}{*}{ Young } & \multirow{2}{*}{$\begin{array}{l}\text { Regular } \\
27.0 \%\end{array}$} & \multirow[t]{2}{*}{ Screening } & \multirow{2}{*}{$\begin{array}{c}\text { Returning } \\
47.0 \%\end{array}$} \\
\hline 1 & MG & $\mathrm{CR}$ & & & & & & \\
\hline 2 & MG & $\mathrm{CR}$ & US & $\mathrm{CR}$ & & $50.3 \%$ & & $26.3 \%$ \\
\hline 3 & MG & $\mathrm{CR}$ & US & NP BI CR & & $3.6 \%$ & $12.3 \%$ & $2.2 \%$ \\
\hline 4 & MG & $\mathrm{CR}$ & US & $3 \mathrm{D} \quad \mathrm{CR}$ & & $3.4 \%$ & & \\
\hline 5 & MG & $\mathrm{CR}$ & $3 \mathrm{D}$ & $\mathrm{CR}$ & & $5.6 \%$ & & $1.3 \%$ \\
\hline 6 & MG & $\mathrm{CR}$ & US & NP & & & $87.7 \%$ & \\
\hline 7 & US & $\mathrm{CR}$ & & & $93.4 \%$ & $6.2 \%$ & & $18.6 \%$ \\
\hline 8 & US & NP & $\mathrm{BI}$ & $\mathrm{CR}$ & $3.6 \%$ & & & \\
\hline 9 & US & MG & $\mathrm{CR}$ & & $3.0 \%$ & & & \\
\hline 10 & MG & $\mathrm{CR}$ & TG & $\mathrm{CR}$ & & $3.9 \%$ & & $2.3 \%$ \\
\hline 11 & MG & $\mathrm{CR}$ & TG & CR US CR & & & & $2.3 \%$ \\
\hline
\end{tabular}

Table 6.4 'Care pathways' of patients at the breast center.

For each patient type, resource and test it is specified whether this test can take place on the resource and how much time this takes for the given patient type. All service times except for biopsies are deterministic, which is in correspondence with both the data and practitioners' opinions. A biopsy takes a uniformly distributed time between 30 and 45 minutes. A consult with the nurse practitioner is 10 minutes for screening patients that tested negative (no cancer), 
and 30 minutes otherwise. For each test it is specified whether the radiologist should be present.

Patients arrive punctually according to the appointment schedule and get assigned a care pathway according to Table 6.4. If their first resource is available, they will commence service immediately, otherwise they will join a queue. Every test type has its own queue, and there is a priority rule among the queues. At service completion at a certain resource, the highest priority queue containing a test type that is compatible with the resource, is checked for non-emptiness. The priority rule for emptying the queues is (high to low priority): CR, biopsy, 3D-ultrasound, nurse practitioner, ultrasound, tomography, mammography. We keep an eventlist of all upcoming arrivals and departures, and store an eventlog to obtain patient and system specific performance measures after the DES run.

Figure 6.7 Screenshot of graphical DES program.

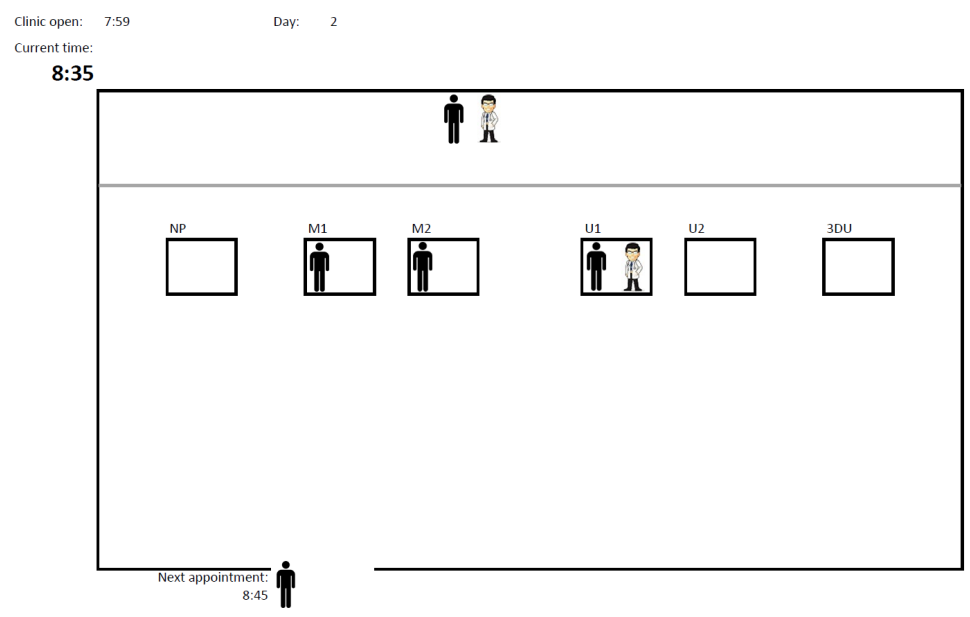

We implemented the DES graphically in Microsoft Excel ${ }^{\circledR}$; software that is commonly available in most Dutch hospitals, and allows for practitioners at the JBH to use and alter the program. A screenshot of the program is depicted in Figure 6.7. In this figure, the squares denote rooms, the white persons are radiologists, and the black persons are patients. Other practitioners are not incorporated in the DES. The rooms are from left to right: the nurse practitioner, two mammography rooms, two ultrasound rooms, and the 3D ultrasound room. The layout of the clinic is similar to the layout of the DES, which provides graphical support in discussing obtained results with practitioners. We additionally implemented a C++ DES for improved random number generation (we used Mersenne Twister [349]) and faster calculations. With the faster calculations we could obtain adequate confidence intervals within acceptable time.

We use common random numbers in order to compare the schedules [294]. Our DES returns the accumulated waiting time for all patients, and for each resource the number of tests performed, the accumulated waiting and idle time between tests performed on this resource, and the last departure time. 
We validated the DES program by letting practitioners observe the graphical simulation and discussing the results of different DES settings with many practitioners. With the graphical DES, the staff was able to validate the movements of patients and radiologists through the center, and provided feedback to make the model more realistic.

Regarding the robustness of the results, we performed several tests with the DES. First, we assumed that the service time durations are not a constant $S$ but uniformly distributed on the interval $[0.9 S, 1.1 S]$. Second, we investigated the effect of non-punctual patients, by assuming that patients arrive a random time from their appointment time. We used a Normal distribution with $\sigma=10$ minutes and three different means: -10, 0, and 10 minutes. The results of the scenarios with early and late arrivals, and with stochastic service durations are displayed in Figures 6.8 and 6.9.

Figure 6.8 The accumulated waiting time per patient for the improved schedule in different scenarios.

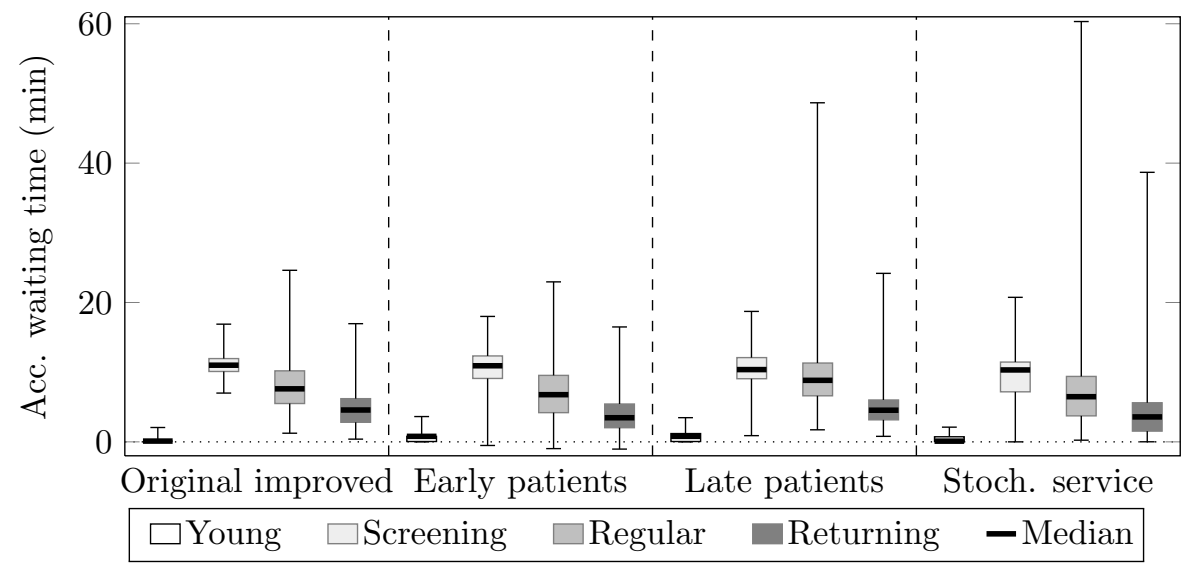


Figure 6.9 Results for the improved schedule in different scenarios for the treatment rooms (f.l.t.r): two mammography rooms, two ultrasound rooms, a 3D ultrasound room and the nurse practitioner.

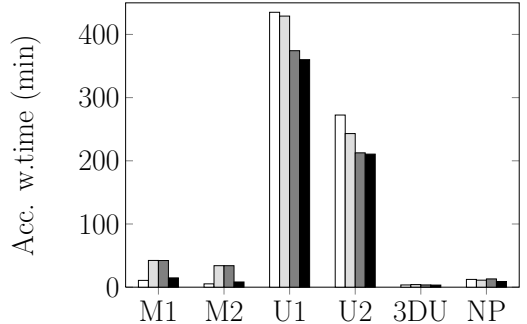

(a)

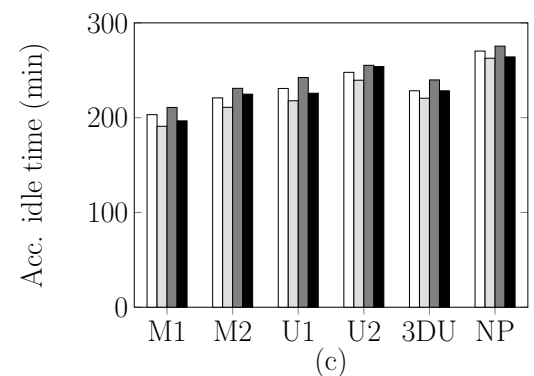

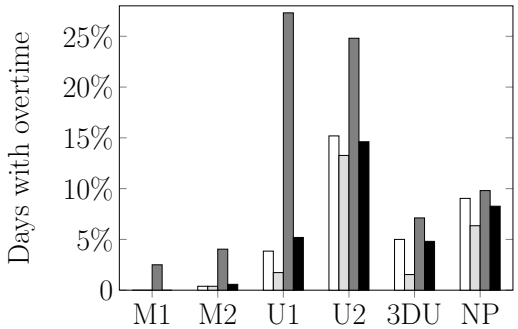

(b)

\footnotetext{
$\square$ Original improved

$\square$ Early patients

$\square$ Late patients

- Stochastic service
} 


\section{Appointments versus walk-in at the plaster room}

\subsection{Introduction}

Patients' waiting time and staff's workload at the plaster room of the JBH fluctuate strongly both throughout each workday and over multiple weekdays. Patients may experience long waiting times, even when they received an appointment for their treatment. The plaster technicians often work in overtime, and the workload fluctuates strongly: congested and quiet times alternate during workdays. In this chapter we present an implementation-oriented case study, in which we provide insight in the workload and possible appointment scenarios at the plaster room.

Typical treatments at a plaster room are applications or removals of casts, splinting and wound checks. Many departments of the hospital depend on the service of the plaster room: patients first get their cast removed at the plaster room, visit a doctor and sometimes radiology, and then get a new cast if necessary. Therefore, long patient waiting times at the plaster room have a knock-on effect on other departments, and may also delay patients that do not require treatment at the plaster room.

Currently, the JBH employs five plaster technicians, of which at least three are present at the plaster room each day. The plaster technicians see patients on both an appointment and a walk-in basis. The technicians share a single appointment system, in which appointments may be scheduled every five minutes. As these appointments are not scheduled with a specific technician, the expected length of the appointment is not taken into account when the patient is scheduled. In the worst case scenario it could therefore occur that at 9:00, 9:05, 9:10, and 9:15 patients are scheduled for treatments that take an hour, which implies that the fourth patient has at least 45 minutes waiting time. However, such long treatment times do not occur often, and data reveals that the probability that a long chain of consecutive time slots is filled is low.

In a system in which more than half of the patients arrive on an appointment based time, a strongly varying workload indicates an inefficient appointment schedule. Unscheduled patients often arrive at the plaster room immediately after a doctor referred them, so the arrival rate depends on doctors' consultation hours. Kortbeek et al. [281] optimize the appointment schedule of an outpatient 
clinic with scheduled and unscheduled arrivals invoking a heuristic. The authors conclude that appointment slots should be scheduled at time periods with relatively few unscheduled patients. For clinics with a single doctor, simulation optimization $[82,465]$ and a local search heuristic [272] were invoked to optimize the appointment schedule, aiming to balance patients' waiting times and doctor idle time and overtime. Additional references on this topic are included in the excellent reviews $[83,242]$ and Chapter 4 . Main difference with existing literature is that at plaster rooms walk-in patients may generally not be rescheduled because they have an appointment with a doctor afterwards. Limiting the daily number of appointments or limiting the daily number of patients seen at the plaster room is therefore not preferred by the JBH.

A plaster room is typically only open during office hours, similar to outpatient clinics. As a consequence, such systems will not evolve long enough to 'reach stationarity', which calls for a transient analysis. In this chapter we therefore analyze different appointment schedules for the plaster room of the JBH invoking discrete event simulation. We include scenarios with increasing patient numbers and different ratios of scheduled and unscheduled patients. The simulation model is described in Section 7.2, and its results in Section 7.3. This chapter ends with the conclusions of this case study.

\subsection{Simulation model}

The aim of this study is to balance the workload throughout each day for the plaster room, and thereby realizing acceptable patient waiting times. This study designs an appointment schedule that achieves this aim, and is easy to implement and work with in practice.

Hospital data shows that the number of patients per day may be approximated by a Poisson distribution, so each simulation day a Poisson random number of arriving patients is drawn. Each patient is a walk-in patient with certain probability, and otherwise receives an appointment. The arrival hour for walk-in patients is determined according to the time dependent arrival rates estimated by the JBH (see Figure 7.1), and the exact arrival time is uniformly distributed within the arrival hour and the next hour of the day. The appointment times are set according to the appointment scheduling rule, which will be varied and described below. We assume that patients are not punctual, i.e., their arrival time at the clinic deviates a random time from their scheduled appointment time; we assume that this deviation is Normally distributed with mean -10 minutes (at the JBH, but also in most outpatient clinics in general, patients tend to arrive early), and $\sigma=10$ minutes.

Each patient has a certain probability $p_{s}$ of being referred by one of the medical specialties $s$, which are: trauma surgery (51\%), orthopedics (35\%), plastic surgery $(11 \%)$, and 'other' $(3 \%)$. This patient type determines from which empirical distribution the service time is drawn. The plaster technicians categorized patients according to their estimated average treatment times into the categories: 
Figure 7.1 Distribution of walk-in patients over the workday.

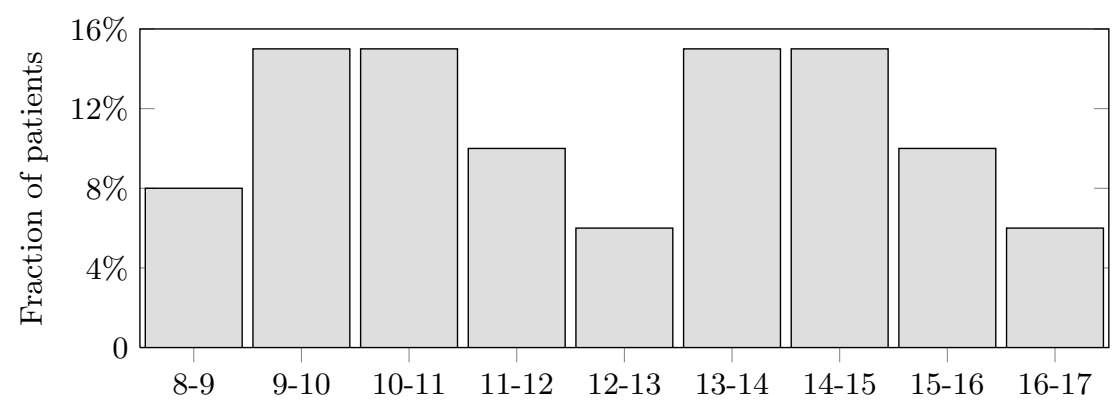

10, 15, 20, 30, 45 or 60 minutes. From hospital data, we obtain the distribution of the patients among the categories, depending on their type, see Figure 7.2. The average treatment time for the total patient population is 15.3 minutes. Let $\mu_{i}$ the estimated treatment time of category $i, i=1, \ldots, 6$. In the simulation model, after the patient's category is determined, each treatment takes a random time, which is uniformly distributed on $\left[0.9 \mu_{i}, 1.1 \mu_{i}\right]$.

Figure 7.2 Distribution of patients over treatment time categories.

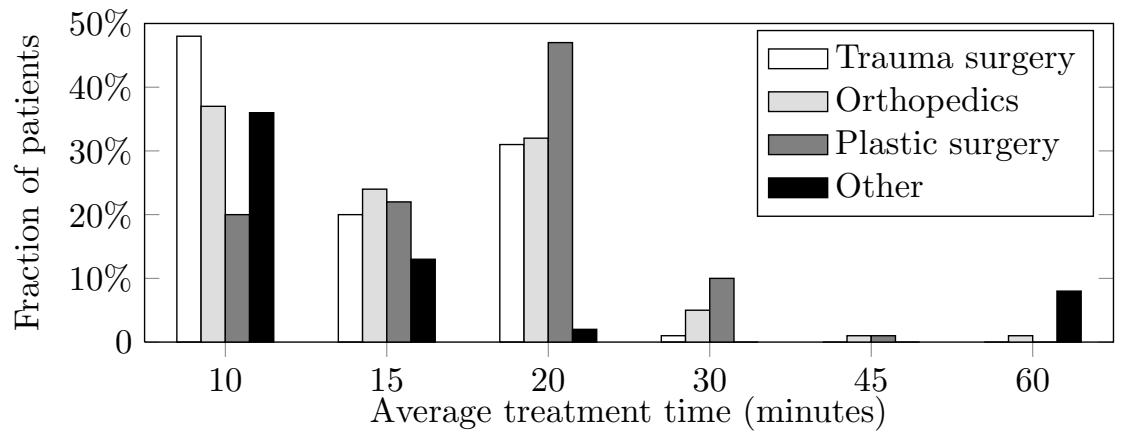

The first plaster technician starts working at 8:00 each day, and the other two start at 8:30. Each technician may take a 30 minutes lunch break, but there should be at least one technician present at the clinic throughout the day. At 17:00 two technicians leave, and the last one stays until all patients from the waiting room are treated.

When a patient arrives at the plaster room and one of the plaster technicians is available, the patient's treatment starts immediately. Otherwise the patient takes place in a waiting room. Currently at the JBH both appointment and walk-in patients are treated in the order of arrival, but the plaster technicians are interested in the effect of prioritizing patients with appointments over unscheduled patients. Therefore, depending on the investigated scenario, we maintain two separate queues for scheduled and unscheduled patients. When a technician completes a treatment, he checks the queue(s) for non-emptiness and starts the treatment of the first patient in the non-empty queue. In case of prioritizing 
scheduled patients, unscheduled patients are only treated when the queue for the scheduled patients is empty.

With the simulation model we investigate several parameter settings. Currently, the plaster room sees on average 52.6 patients per day, but the JBH is interested in the results for both $12 \%$ and $24 \%$ increased arrivals. The utilization of the plaster technicians, which is the average total time patients request treatment divided by the total time available, for the current arrival rate equals $55 \%$. If there would only be two technicians each day, the utilization would be $81 \%$. Therefore, we additionally investigate a scenario with two technicians and the current arrival rate.

The staff estimated which patient types walk-in for their appointments, and $\mathrm{JBH}$ data indicated that with this estimation $62 \%$ of the patients at the plaster room are scheduled in advance. For some of the walk-in patients the referring doctor knows beforehand that the patient requires treatment at the plaster room, but prefers to let the patient choose whether he makes an appointment. If all these patients would also receive appointments, the plaster technicians estimate that in total $76 \%$ of the patients would be scheduled. Therefore, we investigate scenarios with $62 \%$ and $76 \%$ scheduled patients. For each appointment rule, we additionally investigate scenario 'Walk-in (WI)', in which $83 \%$ of the patients walk-in. For the other $17 \%$ of the patients an appointment is either medically necessary, or they require combination appointments with doctors. These appointment patients take one of the available slots randomly, depending on the used appointment rule.

We investigate several appointment scheduling rules in this chapter. Deferring patients to the next day is undesirable for the plaster room, and the plaster technicians always overbook slots to accommodate patients if slots are not available. With the maximum arrival rate investigated, the plaster room requires 96 appointment slots to be able to schedule all patients on $99.99 \%$ of the days without overbooking. The total number of slots available between 8:00 and 17:00 is 108. Based on this insight, and the fact that the average treatment time is slightly over 15 minutes, we design the following appointment rules.

$5 m$ Appointment slots are available every five minutes from 8:00 until 16:55. Arriving patients take one of the available slots randomly. This is the current appointment rule at the plaster room.

5m Appointment slots are available every five minutes from 8:00 until 16:45, but with the slots at 8:05, 8:10, 9:00, 9:25, 9:50, 10:15, 13:00, 13:25, 13:50 and 14:15 closed to prevent waiting and overtime. Arriving patients take one of the available slots randomly.

$10 m$ ' Appointment slots are available every ten minutes from 8:00 until 16:40, but with the slots at 8:10, 9:00, 9:50, 13:00 and 13:50 closed to prevent waiting and overtime. Arriving patients take one of the available slots randomly. If all slots are taken, all non-occupied five minute slots are opened to avoid overbooking. 
A10m Appointment slots are available every ten minutes from 8:00 until 16:40. Arriving patients take alternately the first or last available time slot, to schedule the appointments in the least busy times regarding walk-in patients. The procedure restarts in case of overbooking.

If all slots are occupied, patients are overbooked on a random slot for all appointment rules except rules 10m' and A10m. At any time, at most two patients are scheduled per time slot. Note that the A10m appointment rule has to rely on overbooking more often than the five minute ones. Rule $10 \mathrm{~m}$ ' requires the additional five minute slots on $60 \%$ of the days, and rule A10m overbooks patients on $39 \%$ of the days for the current arrival rate.

The simulation is implemented in $\mathrm{C}++$. In order to apply common random numbers [294] for a better comparison of the schedules, we add all patient arrivals to the eventlist before the start of the day. We simulate 150,000 days as first trials showed large variability between the simulated days. The performance measures of interest are: (1) the waiting time per patient from arrival at the JBH until the treatment starts, so including voluntary waiting time in case the patient arrives early; and (2) the minutes worked in overtime, aggregated over all plaster technicians. In the next section we provide the simulation results and discuss the most interesting insights.

\subsection{Results}

The simulation results indicate that the variability in the waiting and overtime is high, see Figures 7.3-7.8, in which the scale of the upper part of the figure is larger than the scale on the bottom part to display more details of the results. The results of the scenario with a $12 \%$ increased arrival rate are the interpolation of the current arrival rate and $24 \%$ increase, and are therefore omitted.

In the scenarios with three plaster technicians, $50 \%$ of the patients are treated immediately upon arrival. For $75 \%$ of the patients the waiting time is acceptable for all appointment rules in the scenarios with three plaster technicians. On about $75 \%$ of the days the overtime is acceptable for all investigated scenarios and appointment rules. With two technicians the waiting time increases significantly, especially for walk-in patients. However, in all scenarios the maximum value of both performance measures is clearly unacceptable in practice, for all investigated appointment rules. This implies that there are exceptionally busy days and/or times, on which the investigated appointment rules cannot prevent extreme waiting and overtime.

The appointment rule currently used in the JBH, 5m, performs especially bad regarding overtime. Already if ten time slots are closed (rule 5m') the overtime decreases significantly, without affecting the waiting times. For most appointment rules the system performance decreases when $76 \%$ instead of $62 \%$ of the patients make appointments, except for rule A10m. Apparently, achieving that more patients make appointments is not worth the effort for the investigated 
appointment rules. Moreover, especially for the scenario with two plaster technicians, the WI rule performs similar to the other appointment rules. Reducing the number of patients that make appointments reduces the administrative burden significantly, which makes the WI rule attractive to implement.

Figure 7.3 Overtime for different scenarios and appointment rules.

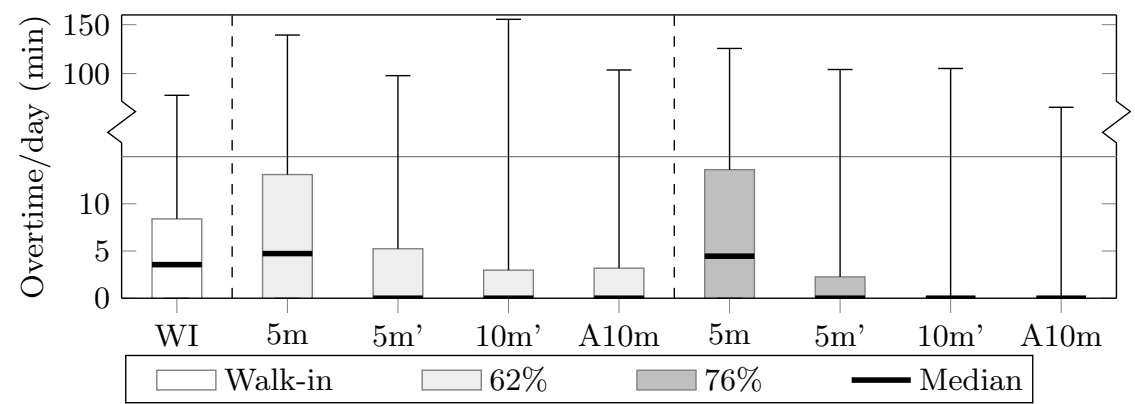

Figure 7.4 Overtime with two plaster technicians.

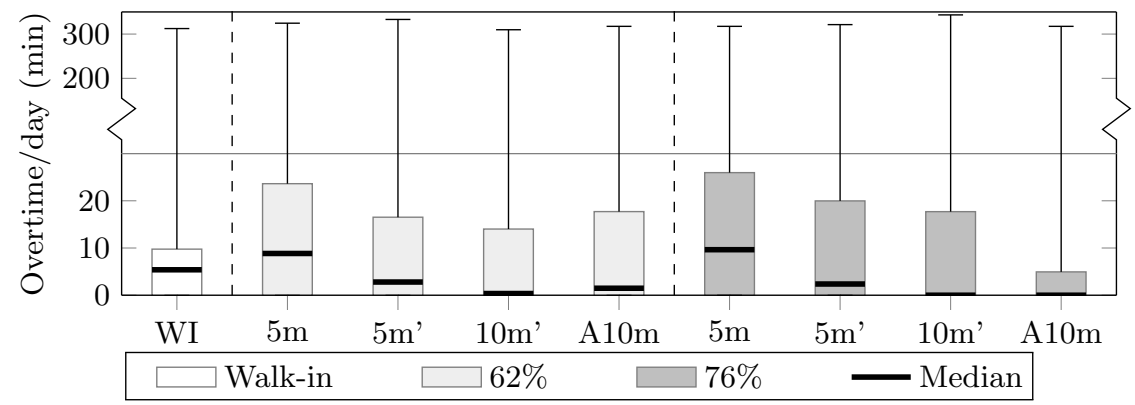

Figure 7.5 Overtime for $24 \%$ more patients.

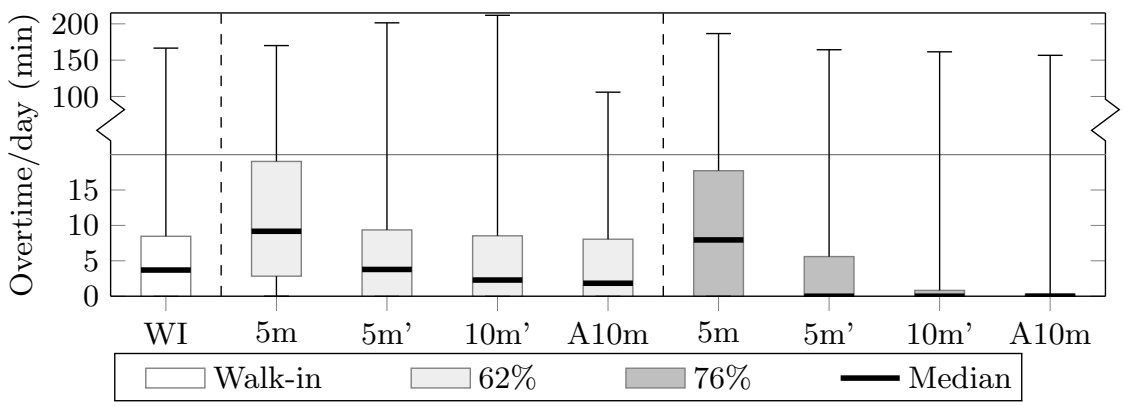

If the JBH would schedule only two plaster technicians each day, patients' waiting times would increase significantly. In this case, the performance of rule A10m is significantly worse than all other appointment rules, especially for walkin patients. For the other appointment rules, $75 \%$ of the patients with appointments wait about 13 minutes or less. If we take into account that patients arrive 
Figure 7.6 Waiting time for different scenarios and appointment rules, with priority for patients with appointment (A) over walk-in patients (NA).

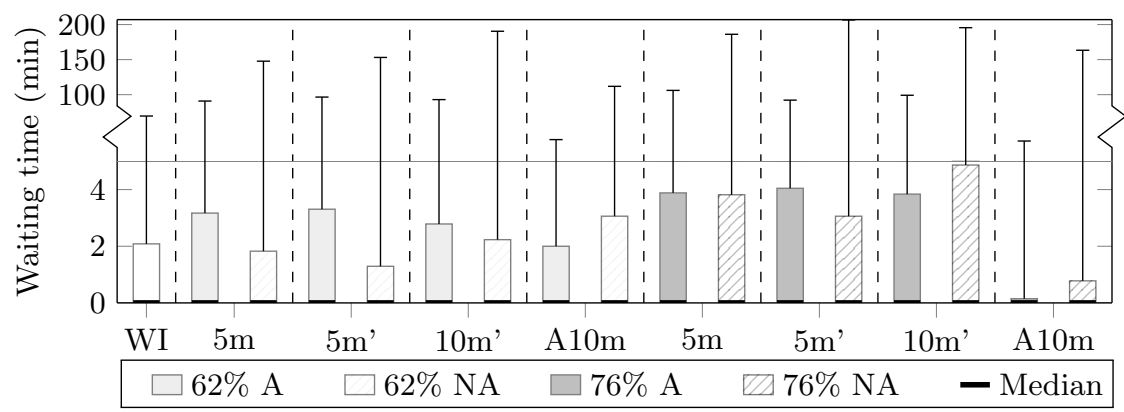

Figure 7.7 Waiting time with two plaster technicians and priority for patients with appointment (A) over walk-in patients (NA).

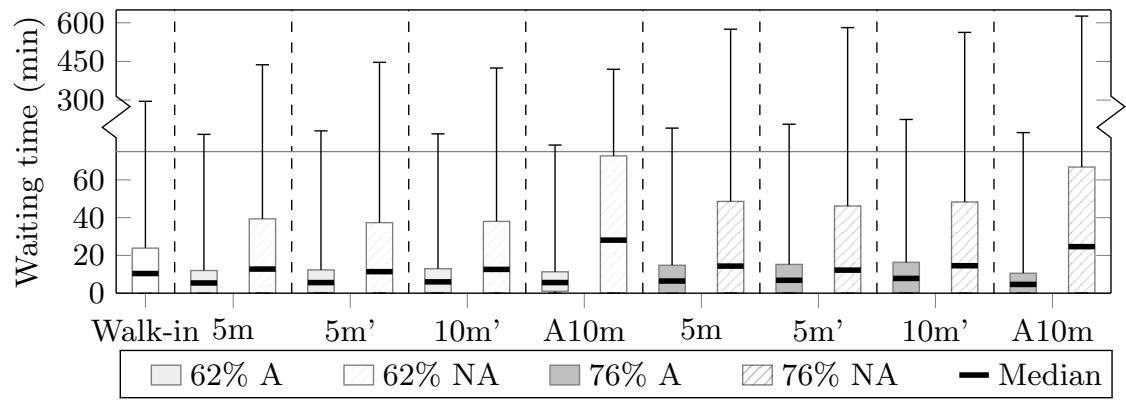

on average ten minutes early, this performance is acceptable. It is generally assumed that walk-in patients are willing to wait longer for their treatment, which was shown to be true for a diagnostic facility at an other Dutch hospital [436], but it requires further research if the performance of $75 \%$ of the patients waiting 40 minutes or less is acceptable.

In all scenarios with three plaster technicians the waiting times for patients with appointment is higher than for walk-in patients. The busier the system

Figure 7.8 Waiting time with $24 \%$ more patients and priority for patients with appointment (A) over walk-in patients (NA).

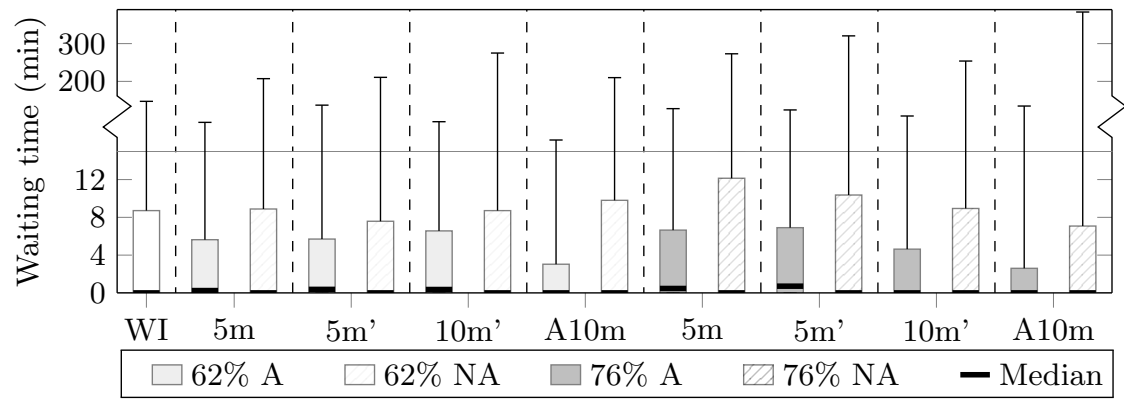


gets, the shorter patients with appointments wait and longer walk-in patients wait. This is because in the investigated scenarios many patients are treated immediately upon arrival, so the priority rule is not applied. In busier systems, the queue with appointment patients will be empty less often, so these patients will be prioritized over walk-in patients more often. The average waiting time without priority rule is equal to the average waiting time in the system with priorities. Therefore, in practice for the current system it will not make a big difference if patients with appointments have priority over walk-in patients.

From a practical viewpoint appointment rules WI, 5m' and 10m' are easy to implement, and the simulation results indicate they will reduce overtime. However, overbooking patients or opening additional slots if necessary is relatively labor-intensive, which makes rule 10m' less practical. Rule A10m results in the best performance for scenarios with three plaster technicians, but is less flexible for patients and requires a central scheduler. Currently, scheduling is decentralized, so this would require significant changes to the system.

In summary, we conclude that appointment rules 5m' and WI would both be promising to reduce overtime, while the waiting time for these appointment rules will be similar to the current rule. Increasing the percentage of patients that make appointments appears not to be worth the efforts. With all investigated appointment rules, the clinic will continue to experience exceptionally busy times with much overtime and long waiting times.

\subsection{Conclusion}

In this chapter we investigated different appointment scheduling rules for the plaster room of the $\mathrm{JBH}$, in an attempt to reduce plaster technicians' overtime and patient waiting time. It appears from the simulation results that waiting and overtime are acceptable for most patients and workdays, but exceptionally high values occur for both performance measures. None of the investigated appointment rules is able to prevent these exceptionally busy times. We did find appointment rules that are both promising to reduce overtime, and easy to implement. These rules also perform well for an increased arrival rate, but might not achieve acceptable performance in case of two plaster technicians. In order to prevent exceptionally busy times, the clinic will have to investigate other interventions, such as offering walk-in patients an appointment at a later time.

This research could be extended by investigating time-dependent waiting times, which possibly reveals bottlenecks throughout the day. With $24 \%$ increased arrival rates all patients can be scheduled without overbooking on $99 \%$ of the days if 14 additional appointment slots are closed in the $5 \mathrm{~m}$ ' appointment rule. Furthermore, we assumed that patients with appointments only have priority over walk-in patients if they are already waiting at the clinic. The plaster technicians would also like to know if they should treat a walk-in patient in case an appointment is scheduled shortly. With such a priority rule, waiting times for appointment patients may decrease, but as the system will have more idle time, 
overtime and walk-in patients' waiting times are likely to increase. Additionally, the results of this research indicate that offering walk-in patients an appointment at busy times might improve the overall performance. It would be interesting to investigate the best policy for offering appointments to walk-in patients, or other possibilities to balance the workload at the plaster room. 



\section{Part IV}

\section{Optimizing doctor schedules}

\section{Chapter 8}

N.M. van de Vrugt, S.T. Luen-English, W.A.P. Bastiaansen, S. Kleinluchtenbeld, W.T.P. Lardinois, M.H. Pots, D.J. Schoonbergen, E.W. Hans, J.L. Hurink, R.J. Boucherie. Simultaneously scheduling tasks and gynecologists to improve patient appointment scheduling. Submitted.

Chapter 9

C.M. Laan, N.M. van de Vrugt, R.J. Boucherie, and J. Olsman. Static and dynamic appointment scheduling to meet access time norms. Submitted. 



\section{8}

\section{Scheduling gynecologists to balance patients' access times}

\subsection{Introduction}

The gynecology department of the Jeroen Bosch Hospital (JBH) experienced difficulties with scheduling outpatient appointments at the medically preferred times. The problem is that pregnant women typically have fixed intervals between two visits to the gynecologist, while the offered number of consultation hours varies from week to week. A gynecologist has, like all other health care professionals, many different tasks in a hospital. Next to seeing patients at the ward, a doctor should be present in the operating theater and outpatient clinic, and leave for study purposes like attending conferences. Making a schedule for these doctors is therefore a difficult job, at the JBH currently performed by one of the gynecologists. This doctor spends two days each six week period to determine an adequate schedule manually, and additionally spends much time to make alterations to the schedule on request of the colleagues.

When creating a schedule, the scheduler has to assign many tasks of different types to shifts, all with their own requirements regarding for example task frequency, and the scheduler additionally has to assign gynecologists to the available shifts in the schedule such that they are able to perform all the tasks assigned to the shifts. The challenges are that there are many constraints on the allowed assignments, e.g., all tasks have different requirements, and some tasks may only be scheduled in a series with other tasks. Despite the time invested in creating the schedule, both the gynecologists and manager of the outpatient clinic see room for improvements in the schedules; gynecologists prefer more compliance with their preferences, and the manager of the outpatient clinic prefers a more balanced number of outpatient clinic hours throughout the weeks. Additionally, the scheduler would prefer to spend less time on creating the schedules.

To support the scheduling of tasks and gynecologists at the JBH, we develop a mixed integer linear program (MIP) approach, which aims to optimize a weighted soft constraint compliance. The MIP combines the assignment of tasks and gynecologists to shifts in a simultaneous decision, and incorporates specializations of the gynecologists that are not strictly increasing in hierarchy levels. Hereby, the requirements of the different task types have to be taken into account, which 
implies that each task of a certain type may only be assigned to a subset of the gynecologists. The MIP takes a prioritized list of hard and soft constraints into account, which is determined by the gynecologists and the outpatient clinic manager. The outcome of the MIP for some test instances forms the base to investigate two heuristic approaches, which are better suitable for practical purposes. Although the MIP is presented for a specific gynecology department, the model is fairly general as many hospital departments have similar constraints on their doctor schedules, and the exact formulation of the constraints is readily tailored to other applications. In the following we highlight some related research. We refer the reader to [490] for a recent review on workforce scheduling.

Gunawan and Lau [208] considered a problem (in a real hospital setting) similar to the one considered in this chapter and also use a mathematical program and a local search heuristic. The authors focus on ergonomic constraints, such as the distribution of physically heavy duties, to ensure a fair schedule. The main difference with the current research, is that gynecologists' specializations are not incorporated. The authors also provide a literature review, to which the reader is referred for additional related references.

In a recent paper, Fügener et al. [178] create a duty schedule for an anesthesiology department. The authors invoke a mixed integer programming approach to first assign shifts to the doctors, and then doctors to workstations. Job qualification levels are incorporated, and are increasing in the number of years of experience and education of the anesthesiologists. In [35], [60] and [515] a similar problem as that of Fügener et al. [178] is treated, but the chosen approaches are different; in [60] a constraint programming approach, in [35] a partial branchand-bounds approach, and in [515] a TABU search heuristic is applied. The problem in the current chapter differs from these papers, as each gynecologist has a (different) subset of tasks she is allowed to perform, and some highly specialized tasks may only be performed by one gynecologist. Fügener et al. [178] provide a literature review, to which the reader is referred for additional related references.

The new contribution of this chapter is that we simultaneously assign tasks and gynecologists to available shifts, and incorporate specializations of the gynecologists that are not strictly increasing in hierarchy levels. We provide pragmatic solutions to a real-world scheduling problem at the gynecology department that are acceptable for the gynecologists, the outpatient clinic management and the patients. The gynecologists and management at the JBH were involved in the entire process, and provided significant input for the developed model and the heuristics.

This chapter is organized as follows. In Section 8.2 we provide the model definition and the heuristics. We end with a presentation of our results in Section 8.3 and a conclusion in Section 8.4. 


\subsection{Model}

In this section we first give an MIP model for the problem of creating suitable schedules for the gynecology department at the JBH. Afterwards we introduce two heuristic approaches for the same problem. Because the MIP formulation is tailored for the gynecology department at the $\mathrm{JBH}$, it may consist of constraints that are not applicable to other departments or hospitals. However, the MIP model is fairly general, as many hospital departments have similar constraints on their doctor schedules, and the exact formulation of the constraints is readily tailored to other applications.

\subsubsection{MIP formulation}

The main decisions in creating a schedule for the gynecology department are to decide during which shifts each gynecologist has to work, and which task types they have to carry out during these shifts. Hereby, preferences of the gynecologists, working regulations, and the requirements of the task types to be performed (e.g., required frequency and skill level) have to be taken into account. To formalize the problem, in the following the sets, parameters and variables required for modeling this scheduling problem are introduced and presented together with the hard and soft constraints and the objective of the MIP.

Let $\mathcal{S}=\{1 m, 1 a, 1 e, 2 m, 2 a, 2 e, \ldots\}$ denote the set of shifts in the planning horizon. Here, the number indicates the day (e.g., ' 1 ' for Monday, '2' for Tuesday, etc.) and the letters ' $m$ ', ' $a$ ' and ' $e$ ' denote the morning, afternoon and evening shift, respectively.

The tasks of the department can be grouped into $N^{T}$ different task types, which set is denoted by $\mathcal{T}=\left\{1, \ldots, N^{T}\right\}$. The tasks have to be performed by a certain number of gynecologists working at the department, which are denoted by a set $\mathcal{G}=\left\{1, \ldots, N^{G}\right\}$. The decision variables describing the schedule after the optimization are denoted by $x_{g s t}$, and are indicators that equal one if gynecologist $g \in \mathcal{G}$ is scheduled for a task of type $t \in \mathcal{T}$ in shift $s \in \mathcal{S}$.

Each gynecologist can be assigned at most one task per shift, which is ensured by the constraint

$$
\sum_{t \in \mathcal{T}} x_{g s t} \leq 1 \quad \forall g \in \mathcal{G}, s \in \mathcal{S} .
$$

There may be task types that cannot be scheduled in all shifts. Therefore, we define set $\mathcal{S}(t) \subset \mathcal{S}$ as the set of shifts in which task type $t$ may be scheduled. Additionally, gynecologists may not always be available due to holidays and other obligations. We define $\mathcal{S}^{h}(g) \subset \mathcal{S}$ as the set of shifts that gynecologist $g$ is unavailable due to holidays or other obligations. Furthermore, each gynecologist is specialized in different tasks, so we define $\mathcal{G}(t) \subset \mathcal{G}$ as the set of gynecologists qualified to perform task $t$. We ensure that each task may only be assigned to 
qualified gynecologists and allowed shifts, by the following constraint.

$$
x_{\text {gst }}=0 \quad \forall g \notin \mathcal{G}(t) \text { or } s \notin\left\{\mathcal{S}(t), \mathcal{S}^{h}(g)\right\} \text { and } t \in \mathcal{T} \text {. }
$$

For each task type a minimum and maximum frequency is specified, denoted by $F_{t}^{\text {min }}$ and $F_{t}^{\max }$, respectively. These frequencies specify how many times this task should/may be scheduled each week. The task 'outpatient surgery' should be scheduled at least once per week and exactly six times every four weeks. To enhance readability, we define subset $\mathcal{S}(j) \subset \mathcal{S}$ to be all shifts of week $j$, $j \in \mathcal{J}=\left\{1,2, \ldots, N^{J}\right\}$. Then, the following constraints set the boundaries for the frequencies of each task type.

$$
\begin{array}{ll}
\sum_{g \in \mathcal{G}} \sum_{s \in \mathcal{S}(j)} x_{g s t} \geq F_{t}^{\text {min }} & \forall j \in \mathcal{J}, t \in \mathcal{T}, \\
\sum_{g \in \mathcal{G}} \sum_{s \in \mathcal{S}(j)} x_{g s t} \leq F_{t}^{\text {max }} & \forall j \in \mathcal{J}, t \in \mathcal{T}, \\
\sum_{g \in \mathcal{G}} \sum_{s \in \bigcup_{i=1}^{4} \mathcal{S}(4 j-i)} x_{g s t}=6 & \forall 4 j \in \mathcal{J}, t=\text { 'outpatient surgery'. }
\end{array}
$$

The task types that refer to on call duty play a special role at the department. Specifically, at any time there should be exactly one gynecologist assigned to the first on call duty, and one to the second on call duty. We define $\mathcal{O} \subset \mathcal{T}$ as the set of task types that refer to on call duties, for example the first and second on call duty task for both weekends and weekdays. In contrast to other task types, tasks of a specific on call type may not be scheduled in parallel. The following constraint ensures this.

$$
\sum_{g \in \mathcal{G}} x_{g s t}=1 \quad \forall s \in \mathcal{S}, t \in \mathcal{O} .
$$

When a gynecologist works an on call duty on a weekday afternoon shift, she must also be assigned an on call duty in the consecutive evening shift. For weekend days the constraint is different, as gynecologists may only be scheduled for on call duty an entire weekend. Let $\mathcal{S}^{m}, \mathcal{S}^{a}, \mathcal{S}^{e}$ denote the subsets of $\mathcal{S}$ containing all shifts corresponding to mornings, afternoons and evenings, respectively. Additionally, define $\mathcal{S}^{w} \subset \mathcal{S}$ as the set of weekend shifts. Let $A(s, n) \in \mathcal{S}$ denote the $n^{\text {th }}$ shift after shift $s$, so for example $A(1 e, 2)=2 a$. Then, the following constraints ensure that on call shifts on weekday afternoons are followed by an evening on call shift, and gynecologists may only be scheduled for on call duty for all six shifts of a weekend.

$$
\begin{array}{ll}
x_{g s t} \leq x_{g s^{\prime} t} & \forall g \in \mathcal{G}, s \in \mathcal{S}^{a} \backslash \mathcal{S}^{w}, s^{\prime}=A(s, 1), t \in \mathcal{O}, \\
x_{g s t}=x_{g s^{\prime} t} & \forall g \in \mathcal{G}, s, s^{\prime} \in \mathcal{S}^{w}, t \in \mathcal{O}, \exists j \text { with } s, s^{\prime} \in S(j) .
\end{array}
$$

Because on call duties are often demanding for gynecologists, after the on call duty shifts a gynecologist is assigned to a task of type 'administration' for one 
morning and afternoon shift. Let $\mathcal{S}^{s e} \subset \mathcal{S}^{w}$ denote the set of evening shifts on all Sundays in the schedule. The following constraints set the administration task type after a weekday and weekend with on call duty.

$$
\begin{aligned}
2 x_{g s t}=x_{g s^{\prime} t^{\prime}}+x_{g s^{\prime \prime} t^{\prime}} \quad \forall g \in \mathcal{G}, s \in\left\{\mathcal{S}^{e} \backslash \mathcal{S}^{w}, \mathcal{S}^{s e}\right\}, s^{\prime}=A(s, 1), \\
s^{\prime \prime}=A(s, 2), t \in \mathcal{O}, t^{\prime}=\text { 'administration'. }
\end{aligned}
$$

Next to all hard constraints on the schedule, the gynecologists have expressed several preferences that would improve the schedule if they were satisfied. To this end, we introduce penalty variables that measure the deviation from the set target. The objective function of the MIP is a weighted sum of the penalty variables. In the following we define several soft constraints and penalty variables.

Besides a minimum and maximum frequency, each task type has a preferred minimum number of times per week it should be scheduled, denoted by $F_{t}^{\text {pref }}$. The deviation from this target for task type $t$ in week $j$ is measured with penalty variable $p_{j t}^{1}$. Furthermore, at the JBH the task type 'education' (in which gynecologists for example educate interns) is preferably scheduled once every two weeks. We introduce penalty variable $p_{j}^{2}$ as the deviation from this frequency. The following constraints ensure the preferred task type frequencies.

$$
\begin{array}{ll}
\sum_{g \in \mathcal{G}} \sum_{s \in \mathcal{S}(j)} x_{g s t}+p_{j t}^{1} \geq F_{t}^{\text {pref }} & \forall j \in \mathcal{J}, t \in \mathcal{T} \\
\sum_{g \in \mathcal{G}} \sum_{s \in \mathcal{S}(2 j)} x_{g s t}+p_{j}^{2} \geq 1 & \forall 2 j \in \mathcal{J}, t=\text { 'education'. }
\end{array}
$$

Each day at the department consists of a morning, afternoon and evening shift. Gynecologists prefer not to have idle time during their workday, e.g., if they are scheduled for a morning and evening shift on a certain day, they prefer to work the afternoon shift too. Recall that the subset $\mathcal{S}^{m}$ consists of all morning shifts, and $A(s, n)$ denotes the $n^{\text {th }}$ shift after shift $s$. We introduce the binary penalty variable $p_{g s}^{3}$, which equals one if gynecologist $g$ has idle time after shift $s$, and the following soft constraint to favor non-idle workdays.

$$
\begin{gathered}
\sum_{t \in \mathcal{T}} x_{g s t}-x_{g s^{\prime} t}+x_{g s^{\prime \prime} t}-p_{g s}^{3} \leq 1 \quad \forall g \in \mathcal{G}, s \in \mathcal{S}^{m}, s^{\prime}=A(s, 1) \\
s^{\prime \prime}=A(s, 2)
\end{gathered}
$$

Furthermore, if a gynecologist is assigned to an on call duty in a certain shift on a weekday, it is preferred that she also works the other on call duty shifts on the same day. This only holds for weekday shifts, as the weekend on call duties are constrained by (8.7) and (8.8). We introduce binary variable $\bar{p}_{g s}^{4}$ that equals one if gynecologist $g$ is assigned at least one on call duty shift on the day (morning, afternoon, and evening shift) containing shift $s$. Additionally, binary penalty variable $p_{g s}^{4}$ equals one if gynecologist $g$ is assigned at least one but less than three on call duty shifts on the day containing shift $s$. The following constraints 
allow to favor schedules in which one on call duty type is assigned to the same gynecologist in all shifts of a single day.

$$
\begin{array}{ll}
x_{g s^{\prime} t} \leq \bar{p}_{g s}^{4} & \forall g \in \mathcal{G}, s \in \mathcal{S}^{m} \backslash \mathcal{S}^{w}, \\
& s^{\prime} \in\{s, A(s, 1), A(s, 2)\}, t \in \mathcal{O}, \\
3 \bar{p}_{g s}^{4}-\sum_{s^{\prime} \in\{s, A(s, 1), A(s, 2)\}} x_{g s^{\prime} t} \leq 2 p_{g s}^{4} & \forall g \in \mathcal{G}, s \in \mathcal{S}^{m} \backslash \mathcal{S}^{w}, t \in \mathcal{O} .
\end{array}
$$

The number of hours a gynecologist is available per week depends on her contracted working hours and holidays, which may be different for each gynecologist. A good schedule takes these factors into account, and assigns approximately the fair number of shifts for each gynecologist with respect to the ratios of contracted hours. We define parameter $R_{g}$ as the ratio of gynecologist $g$ 's contracted working hours to one fte (full-time equivalent). With this parameter we construct parameter $R_{g j}^{s}$, which is the number of shifts gynecologist $g$ should work in week $j \in \mathcal{J}$ according to the contracted working hours. In parameter $R_{g j}^{s}$ we explicitly take holiday weeks into account, to prevent that a gynecologist is assigned to too many shifts surrounding her holiday weeks. It is likely that $R_{g j}^{s}$ contains mostly fractional values, implying that the number of shifts cannot be assigned exactly according to the fte ratios. We quantify 'fairness' in the schedule by the maximum deviation from a desired value. Therefore, we define penalty variables $p_{g j}^{5}$ and $\bar{p}_{g j}^{5}$ as the total number of shifts gynecologist $g$ is under- and over-scheduled in week $j$, respectively. The following soft constraints set these penalty variables.

$$
\begin{array}{ll}
\sum_{s \in \mathcal{S}(j)} \sum_{t \in \mathcal{T}} x_{g s t}+p_{g j}^{5} \geq\left\lfloor R_{g j}^{s}\right\rfloor & \forall g \in \mathcal{G}, j \in \mathcal{J}, \\
\sum_{s \in \mathcal{S}(j)} \sum_{t \in \mathcal{T}} x_{g s t}-\bar{p}_{g j}^{5} \geq\left\lceil R_{g j}^{s}\right\rceil & g \in \mathcal{G}, j \in \mathcal{J} .
\end{array}
$$

Here, $\lceil R\rceil$ and $\lfloor R\rfloor$ denote $R$ rounded up and down to the nearest integer, respectively. Constraints (8.15) and (8.16) would not prevent that one of the gynecologists has the maximum deviation every week, so we additionally introduce two similar constraints for the entire scheduling horizon. Let penalty variables $p_{g}^{6}$ and $\bar{p}_{g}^{6}$ denote the number of shifts gynecologist $g$ is under- and over-scheduled over the entire scheduling horizon, respectively. The following constraints set these penalty values.

$$
\begin{array}{ll}
\sum_{s \in \mathcal{S}} \sum_{t \in \mathcal{T}} x_{g s t}+p_{g}^{6} \geq\left\lfloor\sum_{j \in \mathcal{J}} R_{g j}^{s}\right\rfloor & \forall g \in \mathcal{G}, \\
\sum_{s \in \mathcal{S}} \sum_{t \in \mathcal{T}} x_{g s t}-\bar{p}_{g}^{6} \geq\left\lceil\sum_{j \in \mathcal{J}} R_{g j}^{s}\right\rceil & \forall g \in \mathcal{G} .
\end{array}
$$

At the gynecology department there are many different task types to be performed. The number of times each qualified gynecologist performs each task 
type should be fair with respect to their contracted working hours, and should be balanced for each gynecologist over the entire scheduling horizon. Again, we quantify fairness in the schedule by the maximum deviation from a desired value. Penalty variable $p_{t}^{7}$ measures for each task type $t$ the maximum difference between the number tasks of this type that are performed by gynecologist $g$ and the number of times $g$ is expected to perform task type $t$ according to the fte ratios $R_{g}$. Furthermore, constraint (8.20) ensures that the number of times each task is performed is balanced over the weeks for each gynecologist, where penalty variable $p_{g t}^{8}$ denotes the maximum number of times gynecologist $g$ performs a task of type $t$ in one week.

$$
\begin{array}{ll}
\sum_{s \in \mathcal{S}} x_{g s t} \leq p_{t}^{7} R_{g} & \forall g \in \mathcal{G}, t \in \mathcal{T} \\
\sum_{s \in \mathcal{S}(j)} x_{g s t} \leq p_{g t}^{8} & \forall g \in \mathcal{G}, j \in \mathcal{J}, t=\mathcal{T} \backslash\{\mathcal{O}, \text { administration }\}
\end{array}
$$

Not all soft constraints are equally important to the gynecologists and hospital management. Therefore, for each penalty variable we introduce a penalty parameter $P^{i}$ that reflects the weight of each penalty variable $p^{i}$ in the objective function. Each penalty parameter is specified in the same detail as the accompanying variable is defined, to allow the JBH to set specific preferences if necessary. For example, the preferences of a certain gynecologist, task type, shift, week, or any combination of the indices may be prioritized in the optimization.

All penalty variables and parameters together determine the weighted objective function of this MIP:

$$
\begin{aligned}
& \text { Minimize } \sum_{j \in \mathcal{J}, t \in \mathcal{T}} P_{j t}^{1} p_{j t}^{1}+\sum_{j \in \mathcal{J}} P_{j}^{2} p_{j}^{2}+\sum_{g \in \mathcal{G}, s \in \mathcal{S}^{m}} P_{g s}^{3} p_{g s}^{3} \\
& +\sum_{g \in \mathcal{G}, s \in \mathcal{S}^{m} \backslash \mathcal{S}^{w}} P_{g s}^{4} p_{g s}^{4}+\sum_{g \in \mathcal{G}, j \in \mathcal{J}} P_{g j}^{5}\left(p_{g j}^{5}+\bar{p}_{g j}^{5}\right)+\sum_{g \in \mathcal{G}} P_{g}^{6}\left(p_{g}^{6}+\bar{p}_{g}^{6}\right) \\
& +\sum_{t \in \mathcal{T}} P_{t}^{7} p_{t}^{7}+\sum_{g \in \mathcal{G}, t \in \mathcal{T} \backslash\{\mathcal{O}, \text { admin. }\}} P_{g t}^{8} p_{g t}^{8} .
\end{aligned}
$$

All variables in the MIP are non-negative, with variables $x_{g s t}, p_{g s}^{3}$ and $p_{g s}^{4}$ restricted to binary values. Although this objective function is tailored to the JBH application, the modeling approach allows for integrating diverse types of constraints. As solving the program for realistic scheduling horizons requires substantial running time and specific software that is generally not available in hospitals, in the next subsection we investigate several heuristic approaches to approximate the MIP solution.

\subsubsection{Heuristic approaches}

For practical applications, quickly generating a few good schedules is often preferred over obtaining the optimal schedule in longer computation time [147]. To 
this end, we investigate several local search heuristics, as in many practical applications they have shown to reach high quality solutions and visit many good solutions during their run. In the following, we first clarify how initial solutions for the heuristics are generated, then provide several neighborhood search procedures, and finally describe a multi-start local search and a simulated annealing heuristic. The pseudo-code of the heuristics is provided in Section 8.5.

To apply local search procedures, an initial schedule that complies with all hard constraints has to be generated. The most difficult task types to schedule are the series of on call duties and administrative tasks, as one series involves consecutive shifts. Therefore, we generate the initial schedule by first assigning on call and administrative tasks in a 'weighted random' fashion; for each series of shifts the gynecologists who can perform many other tasks in this series are less likely to be assigned to on call duties. To this end, we weigh the probabilities according to the number of possible task allocations lost by scheduling a gynecologist to a particular set of shifts, which is determined by: (1) the number of different task types the gynecologist is qualified to perform, and (2) the number of shifts the gynecologist is available. Next, we schedule all task types with a minimum scheduling requirement and assign them to gynecologists and shifts following a similar least regret paradigm:

- we choose a task randomly, favoring task types by settings weights that have a relatively high number left to schedule and have fewer possible shifts to be assigned to,

- and we choose a gynecologist/shift combination from the subset of allowed combinations randomly, favoring combinations via weights that allow fewer possible task assignments.

Finally, we schedule the additional outpatient surgery task types randomly, again using weights. These task types have a monthly instead of weekly target and we again favor the gynecologist/shift combinations that allow fewer other task assignments. Note that the scheduling procedure may get stuck without all necessary number of tasks scheduled due to the random choices at each iteration. If at any point there is no allowed assignment for a task, we restart the complete procedure. This procedure appears to generate feasible initial solutions quickly, often using only a few restarts.

The two heuristics described below use a neighborhood search procedure in each iteration. The neighborhood operators investigated are: (1) allocate task, (2) delete task, (3) swap tasks, and (4) reassign on call duties. As the initial schedule contains only the minimum required number of each task type, there are still tasks that may be added after initialization. Tasks may only be deleted if their minimum frequency is not violated. Furthermore, tasks may be swapped, i.e., the gynecologist/shift combination of the two tasks are interchanged, if: (1) the tasks are of different types, (2) both are not on call duties or administrative days, (3) they are scheduled in the same week, and (4) the new gynecologist/shift combination is allowed. Reassigning on call duties requires a series of swaps and several repair operations, as these task types involve a series of consecutive shifts. 
For weekend on call duties, the administrative task shifts of the following Monday are also reassigned. After the reassignment of these series, a repair procedure is initiated that tries to either reschedule or delete all tasks that had to be removed from the schedule before the reassignment. If the repair procedure is not successful, or at any point during this neighborhood operator the resulting schedule would become infeasible, all alterations to the schedule during this iteration are reversed. All developed approaches work only with feasible solutions, to ensure a user can terminate the heuristic at any time and still obtains a feasible schedule.

The neighborhood search procedures are integrated in a multi-start local search (LS) heuristic and a simulated annealing (SA) algorithm. During each iteration in both heuristics one of the neighborhood search procedures is selected randomly, and after its execution a repair procedure is called ensuring that the outpatient surgery is still performed six times each month. The LS heuristic explores the neighborhood of solutions randomly, only accepting new schedules with better objective value, until a certain number of iterations without improvements to the schedule is reached. This solution is then stored, and the heuristic is restarted from a new initial schedule. After a number of restarts depending on the length of the horizon, the heuristic is reapplied to the best found solutions so far for a limited number of iterations. For the SA heuristic, we use the standard acceptance rule and an exponential cooling scheme, see for example [288] for more information.

\subsection{Results}

This section contains the computational and practical results of the MIP model. In Section 8.3.1 we specify the investigated scheduling horizons, the number of gynecologists and tasks, and all input for both heuristics. In Section 8.3.2 we provide insights in both the solution speed and quality of the MIP and the heuristics for different scheduling horizons in a scenario based on data from the JBH. Section 8.3.3 provides a comparison between the best schedule for this scenario and a manual schedule from the $\mathrm{JBH}$, and investigates several scenarios with holiday periods, which are typically hard to solve in practice.

\subsubsection{Case-study specific input}

For the MIP and both heuristics we investigate planning horizons of four, eight, and twelve weeks. This implies $N^{J} \in\{4,8,12\}$. Furthermore, we base the scenarios on data from the JBH. As the JBH currently employs 14 gynecologists, and the department distinguishes 27 different task types, we use these numbers also in our scenarios. The gynecologists and hospital management together determined weight parameters $P^{i}$ for the necessary penalty variables $p^{i}$. In this prioritized list, the task type 'general outpatient clinic' (there are also several specialized outpatient clinic task types) has the highest priority because the JBH experienced difficulties in scheduling patients in the medically preferred weeks 
of their pregnancy. Another example task type with high weight is one of the specialized outpatient clinics, in which the gynecologists cooperate with other specialties. All input for this case study is provided in Section 8.6.

In a first series of tests we consider an instance in which all gynecologists are fully available. As the JBH indicated that holiday periods are generally complicated to schedule, as a second series of tests we consider instances with varying availabilities. Creating good schedules is especially hard during periods in which many gynecologists are unavailable, because each gynecologist has her own specializations and there are specialized tasks that may only be performed by one or few gynecologist(s). This may result in unsolvable instances if all gynecologists for such a task have holidays in the same weeks. However, for holiday weeks there are no penalty scores for over- or under-scheduling a gynecologist as $R_{g s}^{s}=0$ for those weeks. Therefore, it does not hold generally that holiday weeks always result in schedules with higher objective values.

For practical purposes, we limit the solution time of the MIP to one hour, and choose the parameters of the heuristics such that they also terminate within one hour. The MIP was programmed in AIMMS ${ }^{\circledR}$ and solved using CPLEX 12.6.1. Both heuristics were programmed in Python ${ }^{\circledR}$ 2.7. For the LS heuristic, preliminary experiments indicated that limiting the number of iterations without an improved solution to 5000 results in the best balance between exploring new solutions and prematurely judging solutions as 'stuck' in local optima. In the stage of reapplying the LS heuristic to the best solutions initially found by the algorithm, we limit the number of iterations to 100,000, again this proved to be the best compromise between developing many solutions, and sufficiently developing each solution. With the limitation on the running time, this implies that for the four week horizon the heuristic returns three solutions, and for the eight and twelve week horizons two solutions are returned.

In the SA algorithm, based on the results of initial experiments, we set the initial temperature at 300 and use a cooling coefficient of 0.93 , lowering the temperature 100 times each run. This results in a final temperature of about 0.21. The number of iterations possible within the set time frame differed per scheduling horizon, and ranged from 500,000 for the four week planning horizon, to 215,000 for the twelve week planning horizon.

\subsubsection{Computational results of the methods}

The developed methods are only useful in practice if they can deliver good schedules for all practically relevant scenarios within reasonable time. Therefore, in this subsection we first investigate the performance of the MIP and both heuristics for a basic scenario without holidays, which are the easiest to solve in practice.

From our experiments for the basic scenario it appears that all methods are able to deliver a schedule within one hour for all scheduling horizons, see Figure 8.1. It appears that the objective values of the best schedule found with the MIP and both heuristics are close for smaller horizons. For the twelve week horizon the schedules obtained with the heuristics are significantly better than the 
MIP schedule. As expected, for increasing horizon length it becomes increasingly difficult to achieve good solutions within one hour.

Figure 8.1 Analytical results for different horizons.

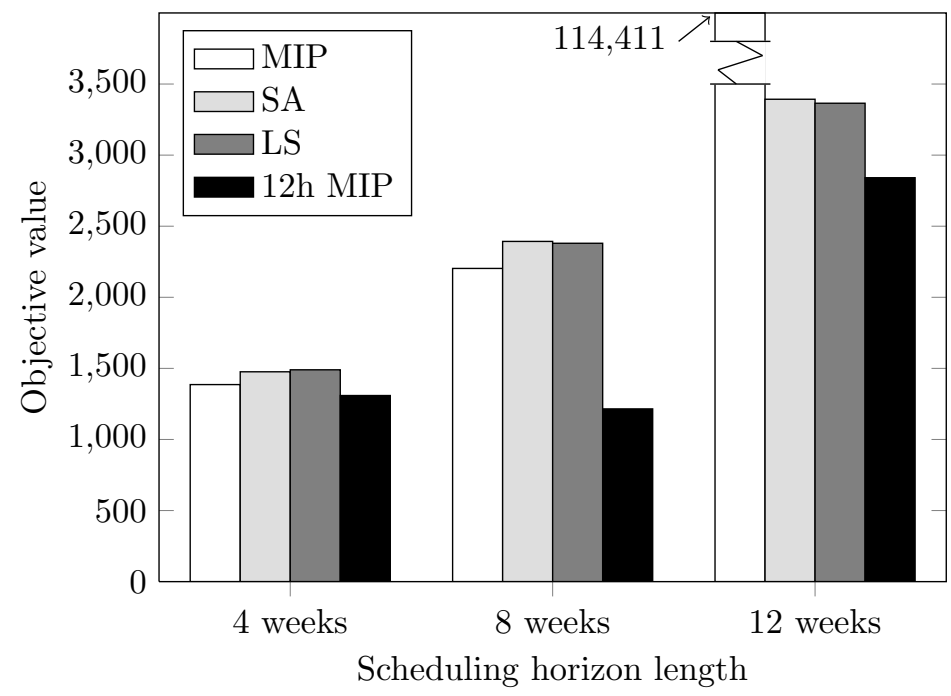

In order to investigate the overall quality of the achieved schedules, we also ran the MIP solver with a time limit of twelve hours, denoted by ' $12 \mathrm{~h}$ MIP' in Figure 8.1. As expected, for the smaller horizons less gain is achieved in the additional eleven hours solving time, but for the twelve week horizon the result improved significantly, even in the last hour of the running time. If we compare the results of the heuristics, we see that for the four and twelve week horizons, the objective values of the schedules found by the heuristics are close to the objective value of the MIP schedule after twelve hour solving time. For the eight week horizon the best MIP solution is significantly better than the solutions obtained with the heuristics.

The above gives an indication of the relative quality of the solutions, but does not indicate their absolute quality. Unfortunately, even after twelve hours of solving time the MIP did not reach the optimal solution for any horizon length, and therefore, we cannot compare the heuristic solutions to the optimal schedules. However, the gaps of the MIP solution after twelve hours provide a lower bound on the optimal objective value. These gaps are still quite large: $25.3 \%, 21.6 \%$, and $20.9 \%$ for the four, eight, and twelve week horizons, respectively. Although this might imply that the optimal schedule has an objective function that is up to $25.3 \%$ lower than the best MIP solution after twelve hours, it can also be the case that the lower bounds used by the solver for the given MIP are quite poor and that the optimal value is much closer to the best achieved solution of the MIP solver. However, considering the structure of the MIP, we conjecture that the latter seems to be more likely. 
Note that the solution found for the eight week horizon has a lower objective value than the solution in the four week horizon. To explain this, we have to take a closer look at the structure of the objective function. In the MIP only the penalty variables $p_{j t}^{1}, p_{j}^{2}$ and $p_{g j}^{5}$ are specified for each week separately, and, thus, lead to an increase if more weeks are considered. However, these penalties have relatively low weights in the objective function. The largest weights in the objective function are for penalty variables $p_{t}^{7}$ and $p_{g t}^{8}$, which are not necessarily higher for a larger number of weeks in the horizon. Additionally, for shorter horizons, not all gynecologists qualified for a certain task will be assigned to perform that task, which may result in larger penalty variables $p_{t}^{7}$ or $p_{g t}^{8}$. Moreover, balancing the number of shifts over the weeks while each gynecologist has to be assigned to a fair number of on call duty tasks, which each consist of many shifts, is harder for shorter horizons.

In summary, we conclude that certainly for larger scheduling horizons, the heuristics perform well compared to the MIP approach with the computation timed to one hour. Furthermore, the two heuristics have similar performance for the investigated scenario.

\subsubsection{Practical results}

Although the performance of the developed methods for the basic scenario appeared promising, a valuable test for the JBH is to compare the computerized schedules to the schedules currently used by the JBH. To be able to make this comparison, we determine the penalty variables for a manually created schedule. However, finding a four week period without any gynecologists on holidays or conferences appeared impossible, and we took a four week schedule from the JBH with: one fte unavailable in week one, $0.75 \mathrm{fte}$ in week three, and $1.7 \mathrm{fte}$ in week four. This schedule did not satisfy hard constraint (8.8) for some combinations of gynecologists and shifts, so these shifts were added to penalty variable $p_{g s}^{4}$. The objective value of this manual schedule was 3057, which should be regarded as an approximation as the schedule did not satisfy all hard constraints. For the four-week period investigated in the previous subsection, which did not have any holidays or conferences, after one hour the MIP reached a value of 1386 . Given that this schedule satisfied all constraints and has a significantly lower objective value, we may conclude that the computerized schedule has great potential for the JBH.

To investigate the performance of our methods in more practically relevant settings, in the following we investigate our methods for different scenarios including summer holiday periods. At the JBH, the summer holiday period consists of six weeks, in which each gynecologist with children may take either the first three weeks (group $\mathcal{G}^{f}$ ) or the last three weeks $\left(\mathcal{G}^{l}\right)$ off. Approximately $1 / 3$ of the gynecologists does not have children $\left(\mathcal{G}^{n}\right)$, and take their three holiday weeks outside this holiday period. At least one of the gynecologists who may perform a specialized task should be available each week.

To test our methodology in holiday periods, we created three test cases for a 
summer holiday period. The three test cases 1,2 , and 3 differ in the distribution of gynecologists over the groups $\mathcal{G}^{f}, \mathcal{G}^{l}$ and $\mathcal{G}^{n}$, and get harder with increasing number. Case 1 is a 'best case scenario', in which all gynecologists allowed to perform highly specialized task types are in $\mathcal{G}^{n}$. For all other task types, we balance the number of gynecologists that may perform this task type evenly over the groups $\mathcal{G}^{f}$ and $\mathcal{G}^{l}$. Case 2 is more difficult to schedule as the gynecologists in $\mathcal{G}^{n}$ are not qualified for the highly specialized task types, but the remaining gynecologists are still spread evenly over other groups according to their specializations. Case 3 is the hardest to schedule, as all gynecologists with the lowest number of contracted fte hours are placed in $\mathcal{G}^{n}$. The other gynecologists are again spread evenly over the other groups. The scheduling period was set to eight weeks, of which the first six were holiday weeks. Again, the solution time was limited to one hour.

Figure 8.2 Practical results for eight week horizon.

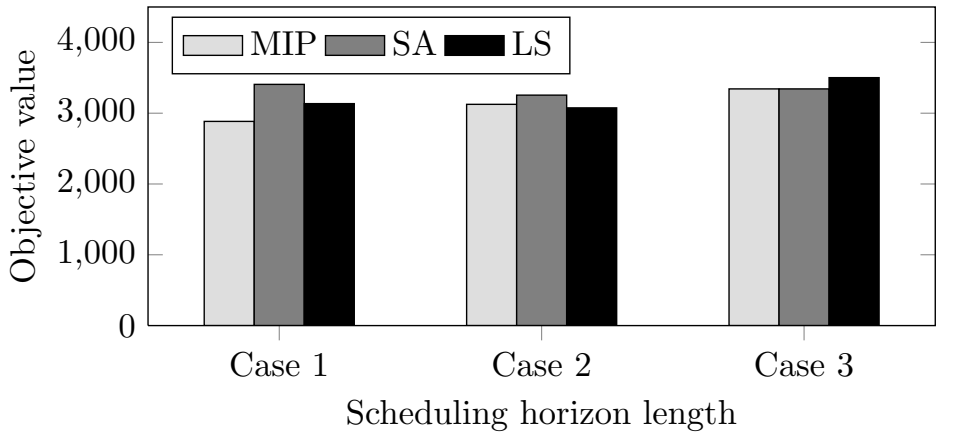

For all three cases, all methods still resulted in feasible schedules, satisfying all hard constraints. The optimized schedules in holiday weeks satisfy, as expected, fewer soft constraints, see Figure 8.2. The objective value of the best MIP schedule without holidays equals 2203 (see Figure 8.1), while for Case 1 it equals 2884, and for Case 3 even 3343. The gap values of the MIP after one hour are $25.2 \%, 24.4 \%$, and $29.6 \%$ for Case 1, 2, and 3, respectively. These gap values are in the same order of magnitude as for the MIP solution for the eight-week horizon after twelve hours running time.

Schedules for periods with and without holidays are difficult to compare. However, the obtained schedules for Case 1 and 2 are still significantly better than the manual (four week) schedule without holidays that was investigated in the previous subsection. This indicates the practical relevance of the schedules in holiday periods for the JBH. Additionally, the difference between the objective values for the hardest Case 3 and best-case scenario Case 1 is relatively small, which is promising for the quality of the schedule for even harder instances. Again, the MIP slightly outperforms the heuristics after one hour solving time, but especially in Cases 2 and 3 the performance of the heuristics is comparable to the MIP model. 
So far, the performance of the investigated methods is assessed through the objective value of the best found schedule. However, in practice it is hard to decide in a fully automated fashion if a schedule is better than another schedule. Despite the time invested in prioritizing the soft constraints, among two schedules with similar objective values gynecologists might have a clear preference, and gynecologists might be indifferent between two schedules with distinct objective values. Although the improvements in the objective value of the schedules are significant, the main practical gain of this research is the significant reduction of manual scheduling time. Furthermore, the benefits of heuristics over an exact approach are that heuristics are particularly useful for providing multiple distinct, good schedules swiftly, so the scheduler has a good starting point for some manual fine-tuning. Both the LS and SA heuristics are therefore very useful in practice, and our numerical experiments show that the performance of both heuristics is good in practical situations. Additionally, creating schedules automatically implies that one gynecologist can be relieved from many hours of scheduling, and the fact that the resulting schedule has a better objective value than the manual schedule is promising for acceptance of the method in practice.

\subsection{Conclusion}

In this chapter we presented a solution approach to simultaneously assigning tasks and gynecologists to shifts for the gynecology department of the JBH. We proposed an MIP model where we incorporated specializations of the gynecologists that are not strictly increasing in hierarchy levels, incorporated hard and soft constraints, and maximized the weighted soft constraint compliance. For practical use, we investigated two heuristics, multi-start local search and simulated annealing, which appeared to provide good solutions, especially for longer scheduling horizons. The presented methods may significantly reduce the time a scheduler has to spend on creating the schedules and improve soft constraint compliance, which may improve for example patients' access time and gynecologists' work satisfaction.

For testing the approach we used an instance based on data from the JBH. Furthermore, the weights used to prioritize the different soft constraints were specified by the gynecologists and the department management of the JBH. After analyzing the obtained schedules it was noticed that often the same tasks were under-scheduled. This may be due to the chosen penalty weights. Therefore, in further research we may adjust the penalties of the soft constraints to also reflect the relative difficulty in satisfying the constraints. This may help to overcome the problem that some tasks are structurally scheduled below their preferred number.

The methods presented in this chapter were all limited to one hour of running time, which is still quite long for practical purposes. It appeared that for the large scheduling horizon the heuristics outperformed the MIP approach and for the smaller scheduling horizons the results were close. However, the investigated 
heuristics were programmed in Python, a programming language known for slow running times compared to certain other languages [403]. Therefore, we believe that implementation in a faster language would imply that heuristics could provide the same solutions in a running time between one and ten minutes, instead of one hour. This would make the heuristics more appealing for practical use.

The MIP formulation presented in this chapter allows for a column generation approach, which would be interesting to explore in future research. For a first step, the two simple heuristics presented in this chapter were more pragmatic in solving the scheduling problem. A further extension to the current research would be to allow the neighborhood search procedures to temporarily visit infeasible solutions, to explore the solution space faster. Additionally, as weekend on call duties should be divided fairly among the gynecologists over a year, incorporating information from the previous results from the MIP (a running horizon approach) would be an interesting extension to the current research.

Concluding, we presented pragmatic solution approaches to a real-world scheduling problem at the gynecology department that are promising for the gynecologists, outpatient clinic management and patients. When we presented the results at the $\mathrm{JBH}$, the department was very interested in using them in practice. 


\subsection{Appendix: Pseudo-code of the heuristics}

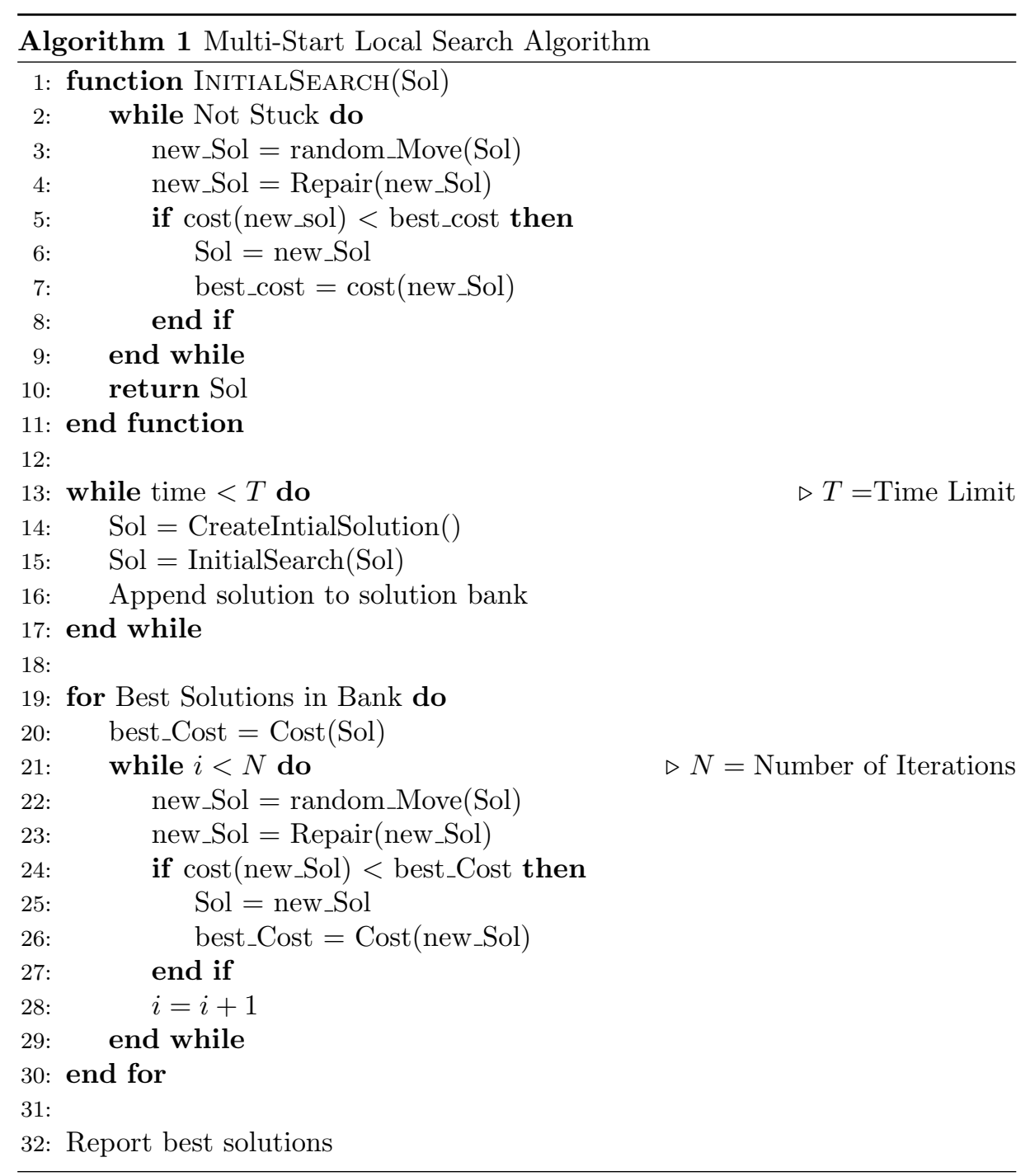




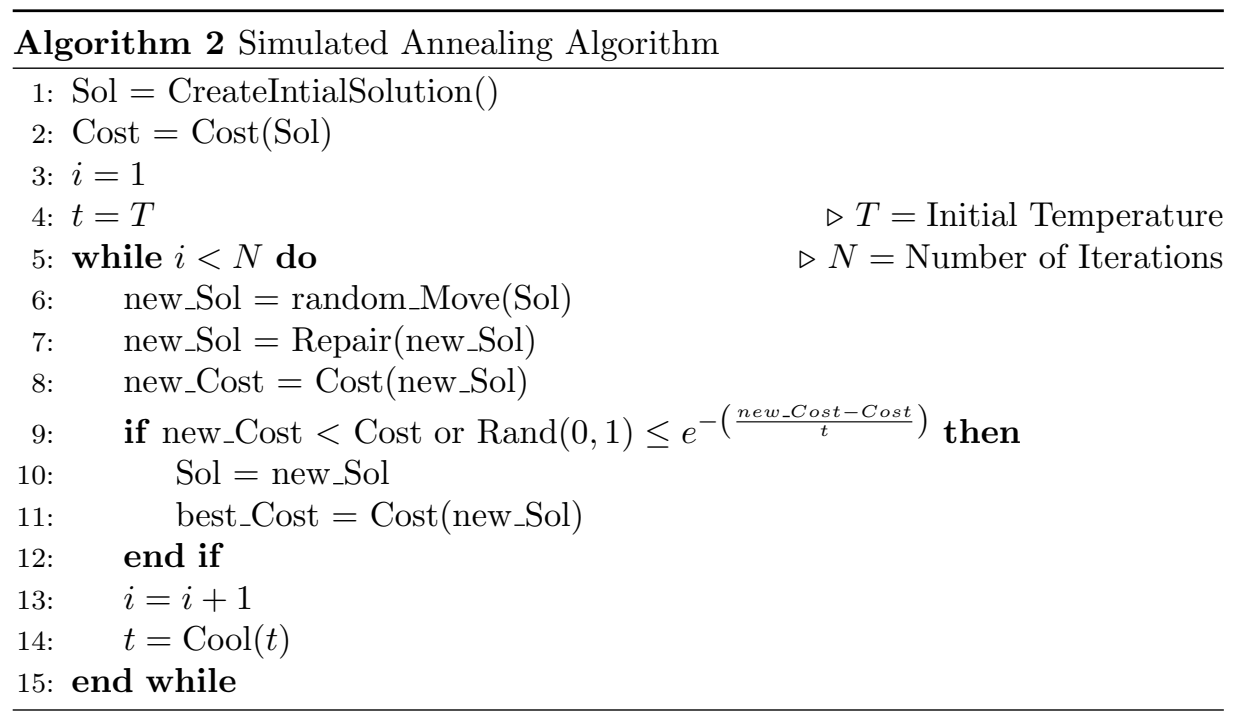

\subsection{Appendix: Parameters for the case study}

In this section we provide all sets and parameters for the JBH case study. Recall that $\mathcal{G}=\{1, \ldots, 14\}$ and $\mathcal{T}=\{1, \ldots, 27\}$. The fte ratios of each gynecologist, $R_{g}$, is given in Table 8.1 .

Table 8.1 $R_{g}$ for each gynecologist.

\begin{tabular}{ll}
\hline$R_{g}$ & Subset of $\mathcal{G}$ \\
\hline 1 & $\{2,5,6,7,9\}$ \\
0.85 & $\{1,3,8,10,11,14\}$ \\
0.75 & $\{4,13\}$ \\
0.6 & $\{12\}$ \\
\hline
\end{tabular}

The gynecologists with $R_{g}<1$ may specify weekday shifts in which they are not available: gynecologists 1, 8, and 13 are not available on all Wednesday shifts; gynecologist 3 is not available on the afternoon and evening shifts on Mondays and Fridays; gynecologist 4 is not available on the morning and afternoon shift on Fridays; and gynecologist 12 is not available on all Monday and Thursday shifts.

The set of gynecologists that is allowed to perform task $t, \mathcal{G}(t)$, is given in Table 8.2. The set of on-call duties is given by $\mathcal{O}=\{\mathrm{D} 1, \mathrm{D} 2, \mathrm{D} 1 \mathrm{w}, \mathrm{D} 2 \mathrm{w}, \mathrm{D} 1 \mathrm{f}$, D2f $\}$.

We assume that the penalty parameters are the same for each week in the schedule. The penalty parameters that do not depend on any index are given in Table 8.3. The task-specific (penalty) parameters are given in Table 8.4. 
Table 8.2 $\mathcal{G}(t)$ for each task.

\begin{tabular}{ll|ll}
\hline Task $t$ & $\mathcal{G}(t)$ & Task $t$ & $\mathcal{G}(t)$ \\
\hline D1 & $\mathcal{G}$ & SE & $\{1\}$ \\
D2 & $\mathcal{G}$ & GV & $\mathcal{G}$ \\
Admin. & $\mathcal{G}$ & GZ & $\{1,11\}$ \\
MA & $\{14\}$ & GR & $\{5,7,14\}$ \\
FE & $\{5,14\}$ & GL & $\{2,6,10,11,13\}$ \\
OK & $\mathcal{G} \backslash\{3\}$ & Outp. surgery & $\{2,6,10,12,14\}$ \\
PI & $\{5,14\}$ & CO & $\{1,7,8,10,11\}$ \\
PO & $\mathcal{G}$ & D1w & $\mathcal{G}$ \\
POb & $\{2,3,4,6,7,9\}$ & D2w & $\mathcal{G}$ \\
AV & $\{1,6-10\}$ & D1f & $\mathcal{G}$ \\
MOC & $\{8,10,11\}$ & D2f & $\mathcal{G}$ \\
BBS & $\{1,5,12,13\}$ & CA & $\mathcal{G}$ \\
PND & $\{3,4,9\}$ & Education & $\{7\}$ \\
EC & $\{3,4,9\}$ & & \\
\hline
\end{tabular}

Table 8.3 Penalty parameters that do not depend on an index.

\begin{tabular}{lccccc}
\hline Parameter & $P_{2}$ & $P_{3}$ & $P_{4}$ & $P_{5}$ & $P_{6}$ \\
Value & 7 & 1 & 50 & 5 & 5 \\
\hline
\end{tabular}


Table 8.4 Task-specific (penalty) parameters

\begin{tabular}{lcccccc}
\hline Task $t$ & $P_{1}$ & $P_{7}$ & $P_{8}$ & $F^{\text {min }}$ & $F^{\text {max }}$ & $F^{\text {pref }}$ \\
\hline D1 & & 20 & 2 & 12 & 12 & 12 \\
D2 & & 20 & 2 & 12 & 12 & 12 \\
Admin. & & & & 24 & 24 & 24 \\
MA & 7 & 5 & 2 & & 2 & 2 \\
FE & 9 & 5 & 2 & & 2 & 2 \\
OK & & 8 & 2 & 10 & 10 & 10 \\
PI & 5 & 5 & 2 & & 3 & 3 \\
PO & 10 & 5 & 2 & 7 & 13 & 10 \\
POb & 6 & 5 & 2 & & 3 & 3 \\
AV & 9 & 4 & 2 & & 1 & 1 \\
MOC & 10 & 5 & 2 & & 1 & 1 \\
BBS & 6 & 5 & 2 & & 1 & 1 \\
PND & 6 & 5 & 2 & & 1 & 1 \\
EC & 6 & 5 & 2 & & 3 & 3 \\
SE & 8 & 5 & 2 & & 1 & 1 \\
GV & 6 & 5 & 2 & & 1 & 1 \\
GZ & 7 & 5 & 2 & & 2 & 2 \\
GR & 8 & 5 & 2 & & 3 & 3 \\
GL & 7 & 5 & 2 & & 4 & 4 \\
Outp. surgery & & 8 & 2 & 1 & 6 & 1 \\
CO & & 8 & 2 & 2 & 2 & 2 \\
D1w & & 20 & & 6 & 6 & 6 \\
D2w & & 20 & & 6 & 6 & 6 \\
D1f & & 20 & & 3 & 3 & 3 \\
D2f & & 20 & & 3 & 3 & 3 \\
CA & 8 & 5 & 2 & & 1 & 1 \\
Education & & 5 & & & 1 & 1 \\
\hline
\end{tabular}


Recall that $\mathcal{S}(t)$ denotes the set of shifts when task $t$ can be performed, and $\mathcal{S}^{i}$ for $i \in\{w, m, a, e\}$ denotes the set of weekend, morning, afternoon and evening shifts, respectively. For the ease of notation, we additionally introduce $\mathcal{S}^{i}$ for $i \in\{M, T u, W, T h, F\}$, which denote all shifts on Monday, Tuesday, Wednesday, Thursday, and Friday, respectively. Then, Table 8.5 gives $\mathcal{S}(t)$ for each task $t$.

Table 8.5 $\mathcal{S}(t)$ for each task $t$.

\begin{tabular}{|c|c|}
\hline Task $t$ & $\mathcal{S}(t)$ \\
\hline D1 & $\mathcal{S} \backslash\left\{\mathcal{S}^{w}, \mathcal{S}^{F}\right\}$ \\
\hline D2 & $\mathcal{S} \backslash\left\{\mathcal{S}^{w}, \mathcal{S}^{F}\right\}$ \\
\hline Admin. & $\mathcal{S} \backslash\left\{\mathcal{S}^{w}, \mathcal{S}^{e}\right\}$ \\
\hline MA & $\mathcal{S} \backslash\left\{\mathcal{S}^{w}, \mathcal{S}^{e}\right\}$ \\
\hline $\mathrm{FE}$ & $\mathcal{S}^{T u} \backslash \mathcal{S}^{e}$ \\
\hline OK & $\mathcal{S} \backslash\left\{\mathcal{S}^{w}, \mathcal{S}^{e}\right\}$ \\
\hline PI & $\mathcal{S}^{m} \cap\left\{\mathcal{S}^{M}, \mathcal{S}^{T u}, \mathcal{S}^{T h}\right\}$ \\
\hline $\mathrm{PO}$ & $\mathcal{S} \backslash\left\{\mathcal{S}^{w}, \mathcal{S}^{e}\right\}$ \\
\hline $\mathrm{POb}$ & $\mathcal{S} \backslash\left\{\mathcal{S}^{w}, \mathcal{S}^{e}\right\}$ \\
\hline AV & $\mathcal{S}^{T u} \cap \mathcal{S}^{e}$ \\
\hline $\mathrm{MOC}$ & $\mathcal{S}^{M} \cap \mathcal{S}^{m}$ \\
\hline BBS & $\mathcal{S}^{T u} \cap \mathcal{S}^{a}$ \\
\hline PND & $\mathcal{S}^{M} \cap \mathcal{S}^{m}$ \\
\hline $\mathrm{EC}$ & $\mathcal{S}^{T h} \cap \mathcal{S}^{m}$ \\
\hline $\mathrm{SE}$ & $\mathcal{S}^{T u} \cap \mathcal{S}^{e}$ \\
\hline GV & $\mathcal{S}^{W} \cap \mathcal{S}^{m}$ \\
\hline GZ & $\mathcal{S}^{M} \backslash \mathcal{S}^{e}$ \\
\hline GR & $\left\{\mathcal{S}^{W}, \mathcal{S}^{T h}\right\} \backslash \mathcal{S}^{e}$ \\
\hline GL & $\left\{\mathcal{S}^{T u}, \mathcal{S}^{W}, \mathcal{S}^{F}\right\} \backslash \mathcal{S}^{e}$ \\
\hline Outp. surgery & $\left\{\mathcal{S}^{M} \cap \mathcal{S}^{m}, \mathcal{S}^{F} \cap \mathcal{S}^{a}\right\}$ \\
\hline $\mathrm{CO}$ & $\left\{\mathcal{S}^{T u} \backslash \mathcal{S}^{e}, \mathcal{S}^{T h} \cap \mathcal{S}^{m}\right\}$ \\
\hline $\mathrm{D} 1 \mathrm{w}$ & $\mathcal{S}^{w}$ \\
\hline $\mathrm{D} 2 \mathrm{w}$ & $\mathcal{S}^{w}$ \\
\hline D1f & $\mathcal{S}^{F}$ \\
\hline D2f & $\mathcal{S}^{F}$ \\
\hline $\mathrm{CA}$ & $\mathcal{S}^{F} \cap \mathcal{S}^{a}$ \\
\hline Education & $\mathcal{S} \backslash\left\{\mathcal{S}^{w}, \mathcal{S}^{e}\right\}$ \\
\hline
\end{tabular}

The subsets of gynecologists that are used in the summer holiday test cases are given in Table 8.6.

Table 8.6 Subsets of $\mathcal{G}$ for the summer holiday cases.

\begin{tabular}{lccc}
\hline Case & $\mathcal{G}^{f}$ & $\mathcal{G}^{l}$ & $\mathcal{G}^{n}$ \\
\hline 1 & $\{2,3,8,12\}$ & $\{4,6,9,10,13\}$ & $\{1,5,7,11,14\}$ \\
2 & $\{2,5,8,9\}$ & $\{3,4,10,11,13\}$ & $\{1,6,7,12,14\}$ \\
3 & $\{2,5,6,7,8\}$ & $\{1,9,10,11,14\}$ & $\{3,4,12,13\}$ \\
\hline
\end{tabular}




\section{9}

\section{Static and dynamic surgeon scheduling}

\subsection{Introduction}

Appointment scheduling systems for outpatient clinics have great influence on hospital efficiency, and can additionally provide patients with timely access to health care services [212]. Timely access is important for both medical outcomes and patient satisfaction. To this end, in the Netherlands outpatient clinics have to comply with national access time norms [61], which impose a maximum on the time between requesting an appointment and the appointment itself.

Many outpatient clinics make tactical appointment schedules in which the available capacity is divided among all patient types [242]. This tactical schedule determines to a large extent patients' access times, as patients often get the first available slot for their type when they request an appointment. However, the number of arriving patients fluctuates throughout the year, and the availability of the doctors is not constant due to other obligations and holidays. As a consequence, overbooking is often unavoidable to provide patients with reasonable access times. Overbooking may result in doctors working overtime, and in large waiting times for patients.

The situation as described above is also found at the surgical outpatient clinic of the JBH. The number of arriving patients is related to, for example, the weather conditions (more bone fractures in icy weather) and the day of the week during which the arrival takes place (on Mondays more sports injuries). The staff at the surgical outpatient clinic experiences large difficulties in coping with the varying demand, and is increasingly relying on overbooking the clinic's sessions to meet their patient's access time norms. Moreover, the capacity at the clinic varies because surgeons have other obligations and holidays. The research presented in this chapter is motivated by the questions of the JBH: what is the minimally required capacity to comply with the access time norm, and how could the outpatient clinic's appointment scheduling be made more robust with respect to the variance in demand and capacity?

Appointment scheduling in healthcare has received considerable attention from academics in the past years. In this chapter we summarize the most related results, and refer the reader to the reviews [83, 212, 242] and Chapter 4 for a broader view of this field. The objective of this study is to optimize the 
tactical appointment schedule with respect to access time and idle time, in such a way that the performance is robust against varying demand and supply. We found three papers studying access time improvements at outpatient clinics at the tactical planning level. Joustra et al. [255] determine an appointment schedule for one week using linear programming, and evaluate patients' access times and the number of overbooked appointments for that schedule using simulation. If necessary, the linear program is run again with input based on the simulation results; these iterative steps are repeated until the schedule complies with all norms. Elkhuizen et al. [159] obtain global insights into the required capacity to meet the access time norms by means of a queueing model, and evaluate the performance of the clinic in more detail by means of a simulation model. Creemers et al. [128] invoke a bulk-server queueing model to evaluate access times, and use complete enumeration to determine the best assignment of the capacity to different patient types.

Dynamic capacity allocation typically includes reserving capacity for urgent or walk-in patients, or obtaining policies that describe how many patients of each diagnostic group should be admitted into the hospital from a waiting list to optimize utilization and access times, cf. [243, 276]. Several studies evaluate specific scheduling policies for reserving capacity for different patient types by means of simulation, cf. [269, 504].

A different, but related topic is the master surgery scheduling problem, in which tactical schedules assign operating theater time to all (sub-)specialties in a hospital [381]. This field of literature typically studies the objective of balancing the occupancy of post-operative wards, which differs from our objective. Moreover, only a few papers incorporate uncertain demand; for example Holte and Mannino [233] minimize the (weighted) queue lengths of different patient types for a cyclical schedule with stochastic demand.

In this chapter, we present a new stochastic mixed integer programming model (SMIP) to optimize a tactical appointment schedule with respect to access times. We approximate the SMIP with two different approaches: (1) a new deterministic mixed integer programming approach, which results in a static appointment schedule, and (2) a new Markov decision model approach, which results in a dynamic scheduling strategy. The approaches are fitted to the two questions the JBH posed, and result in practically relevant schedules. We evaluate the performance of the methods with discrete event simulation. Additionally, we include a numerical analysis, based on the discrete event simulation, that helped the JBH assess the benefits of the different schedules. The models developed in this chapter can readily be adapted to other outpatient clinics and appointment systems in other applications than healthcare.

\subsection{Mathematical model}

The model presented in this chapter mimics a typical Dutch outpatient clinic that uses a tactical appointment schedule in which a fixed capacity (number of 
appointment slots) is reserved for each patient type each week. The clinic works with a block schedule, in which a morning or an afternoon block consists of a specific number of time slots that are reserved for certain patient types. There may be multiple blocks scheduled on the same day part, as multiple surgeons can work in parallel.

Once set, each block type (morning or afternoon) is not allowed to change often, due to surgeon preferences and technical limitations. In general, if a surgeon is not available an entire block is canceled, and if access times exceed the norm surgeons work overtime or add an entire block to the schedule. Therefore, the composition of the blocks, i.e., how many appointment slots for each patient type, play a significant role in patients' access times. As working overtime involves much additional scheduling efforts and often increases patient waiting times, we determine the minimally required number of scheduled blocks and each block type's composition without surgeons working overtime. To this end, we present a stochastic mixed integer programming model (SMIP) that incorporates stochastic arrivals and capacity. We approximate the SMIP's solution in two ways: (1) a MIP in which we incorporate stochastic arrivals but assume that the capacity is fixed, which results in the optimal static schedule, and (2) a Markov decision model, which results in the optimal flexible scheduling policy. The SMIP and both approximation approaches are presented in this section.

\subsubsection{The stochastic scheduling problem}

In accordance with the JBH's current practice, we develop a cyclic tactical schedule that distributes the capacity among the different patient types. A SMIP is invoked to decide on both the number of scheduled blocks and the number of appointment slots that are planned for each patient type during each block. The objective of the SMIP is to balance patient access time and doctor idle time.

In the following, we define the sets, parameters, variables and constraints of the SMIP. Let $p \in \mathcal{P}=\{1, \ldots, P\}$ denote the patient types, $d \in \mathcal{D}=\{1, \ldots, D\}$ the days, and $b \in\{$ morning, afternoon $\}$ the block types. In the SMIP it is possible to schedule more days than the number of clinic days in the cycle, because there are multiple surgeons who may work in parallel. The parameters and variables are denoted as follows.

$L_{p} \quad$ appointment length for type $p$ (number of time slots)

$c_{b} \quad$ capacity of block $b$ (number of time slots)

$M^{b} \quad$ maximum number of blocks during one cycle

$C^{a} \quad$ cost for having an access time of one day

$C^{e} \quad$ cost for having an empty time slot

$X_{p d b}$ number of type $p$ patients scheduled on day $d$, block $b$

$Y_{d b}$ indicator that equals 1 if patients are scheduled on day $d$, block $b$

$c_{p}^{t} \quad$ total number of appointment slots scheduled for type $p$

The maximum number of blocks during one cycle, $M^{b}$, is used to limit the solution space of the SMIP, and is set sufficiently large to accommodate all demand. The cost parameters $C^{a}$ and $C^{e}$ can be used to give priority to minimizing either 
the access time or the idle slots, and can be chosen according to the preferences of the hospital. Note that the parameters and variables of the SMIP are not stochastic. As in practice not the schedule itself, but the realization of the schedule and the patient arrivals are stochastic, we formulated the SMIP such that the stochasticity is only incorporated in the objective function.

The following constraints hold for the parameters and variables.

$$
\begin{array}{ll}
c_{p}^{t}=\sum_{d, b} X_{p d b} & \forall p \\
\sum_{p} X_{p d b} \cdot L_{p}=c_{b} & \forall d, b \\
\sum_{d, b} Y_{d b} \leq M^{b} & \\
X_{p d b} \leq Y_{d b} \cdot c_{b} & \forall p, d, b \\
X_{p d^{\prime} b} \leq X_{p d b}+1 & \forall p, d, d^{\prime}, b \\
X_{p d^{\prime} b} \geq X_{p d b}-1 & \forall p, d, d^{\prime}, b \\
\sum_{d} Y_{d b} \leq \sum_{d} Y_{d b^{\prime}}+1 & \forall b, b^{\prime} \\
\sum_{d} Y_{d b} \geq \sum_{d} Y_{d b^{\prime}}-1 & \forall b, b^{\prime} \\
c_{p}^{t}, X_{p d b} \in \mathbb{N} & \forall p, d, b \\
Y_{d b} \in\{0,1\} & \forall d, b
\end{array}
$$

The first constraint (9.1) sets $c_{p}^{t}$, which is used to calculate the average access time. We assume the total number of appointment slots is distributed evenly over the days in the cycle, as we cannot incorporate time-dependent arrival rates due to lack of data. Constraints (9.2) and (9.3) ensure that the available capacity is not exceeded, and all time slots of each block are used in the schedule. Constraint (9.4) makes sure that a surgeon is scheduled when patients are scheduled on that day. Often in practice, the capacity of block $b$ also depends on the day of the week; this could readily be incorporated in the model and would only affect constraints (9.3) and (9.4), but as the JBH requires the block sizes to equal for each day this is not incorporated in the SMIP. Constraints (9.5)-(9.8) are not necessary for the SMIP, but reflect preferences of the JBH. Constraints (9.5) and (9.6) balance the workload over the surgeons by requiring that the number of scheduled patients on each block differs at most one. Constraints (9.7) and (9.8) ensure that the number of morning and afternoon blocks are balanced, such that on most days both a morning and afternoon block is scheduled. The last constraints (9.9) and (9.10) define the variable types.

The SMIP formulation above does not include stochastic parameters or variables, and is not related to the demand of the clinic. We incorporate the stochas- 
tic capacity and demand in the objective function:

$$
\min C^{a} \sum_{p} f^{a}\left(c_{p}^{t} \mid \lambda_{p}, u\right)+C^{e} \sum_{p} f^{e}\left(c_{p}^{t} \mid \lambda_{p}, u\right) .
$$

Here, parameter $u \in[0,1]$ denotes the cancellation probability of the clinic, and $\lambda_{p}$ the arrival rate of type $p$ patients. Therefore, $f^{a}\left(c_{p}^{t} \mid \lambda_{p}, u\right)$ and $f^{e}\left(c_{p}^{t} \mid \lambda_{p}, u\right)$ are stochastic variables. Furthermore, the functions $f^{a}$ and $f^{e}$ relate the stochastic total realized number of appointment slots and arrival distribution for each patient type to the expected access time and number of empty slots, respectively. These formulas cannot be obtained exactly, but are approximated in Section 9.2.2. Note that this objective allows to set weights for each patient type individually, if necessary.

In this SMIP, both the demand and the capacity are stochastic. There exist several approaches for solving SMIPs, for example decomposition algorithms, cf. [441], and robust optimization approaches, cf. [45]. In this chapter we solve the SMIP with a fixed and a flexible approximation method; for the first method we assume that the capacity is deterministic and solve a MIP, and for the second method we approximate the schedule using Markov decision theory. These methods are both practically relevant and offer the JBH two distinct alternatives with good performance. We describe the methods in the following two subsections.

\subsubsection{Fixed scheduling}

The stochasticity in the SMIP makes that we cannot directly solve the model as presented above. We first approximate the SMIP by a (deterministic) mixed integer program (MIP) in which we assume that the cancellation probability at the clinic $u$ is equal to the average cancellation probability. We incorporate the stochasticity in the patient arrivals in the MIP by means of a discrete time queueing model. The queueing model relates the deterministic realized capacity to the expected access time and number of idle slots for each patient type. In the following we clarify the queueing model and the necessary adjustments to convert the SMIP into a MIP.

The discrete time queueing model is presented analogy with the ones presented in $[281,346]$. The state of the model is the size of the backlog of a specific patient type $p$ at the end of day $d, B_{d p}$. Every day a number of patients, at most equal to the capacity $c_{d p}$ for this type on this day, is removed from the backlog, and a random number of new patients, $A_{d p}$, is added to the backlog. We do not allow arriving patients to be scheduled on the same day. For the backlog on day $d$, we have:

$$
B_{d p}=\left(B_{d-1, p}-c_{d p}\right)^{+}+A_{d p},
$$

in which $x^{+}=x$ if $x>0$ and $x^{+}=0$ otherwise. Eq. (9.11) is known as Lindley's recursion (cf. [116]). By means of the queueing model we derive a relation between the daily capacity and expected access and idle times by calculating the stationary 
distribution of $B_{d p}$. We assume that the total realized capacity is divided equally over the weekdays. Therefore, the conversion from $c_{p}^{t}$ to $c_{d p}$ is $c_{d p}=\left\lfloor c_{p}^{t} / D\right\rfloor$, with $\lfloor x\rfloor$ denoting rounding down to the nearest integer, and the remaining $c_{p}^{t}-D$. $\left\lfloor c_{p}^{t} / D\right\rfloor$ days added to days with the lowest index and enough surgeons available. Section 9.5 presents more details on the queueing model; the exact definition of $f^{a}$ is given by (9.13), and for $f^{e}$ by (9.12).

To be able to incorporate functions in the objective of the MIP, we introduce parameters $m_{p}$ and $M_{p}$ denoting the minimum and maximum number of appointment slots scheduled for type $p$, respectively. Before solving the MIP we create an array with $f^{a}$ and $f^{e}$ evaluated for each patient type and all possible numbers of slots between $m_{p}$ and $M_{p}$. We assume parameter $m_{p}$ is larger than the average arrival rate of type $p$ patients, otherwise the queueing model is not solvable. Furthermore, $M_{p}$ is set sufficiently large to accommodate all arrivals, and we introduce additional constraints to ensure that $m_{p} \leq T_{p} \leq M_{p}$.

Note that the model formulation of both the queueing model and the MIP allow to incorporate other performance measures than the expected values, for example the probability that the access time norm is met for $90 \%$ of the patients. The JBH preferred the average performance measures, as more robust schedules are likely to require more capacity, which is not available in practice.

The MIP provides both the number of blocks that should be scheduled each week, and the number of appointment slots in each block type for each patient type. This schedule is the base scenario for our numerical study. If the variance in the capacity of the clinic is relatively low, the schedule obtained with the MIP should result in acceptable performance in practice. In order to accommodate for higher variability, in the following subsection we present a flexible scheduling approach, which is based on the MIP schedule.

\subsubsection{Flexible scheduling}

Since both the capacity and the number of patients requesting an appointment fluctuates during the year, the performance of the clinic will improve by allocating capacity dynamically. The basis of this flexible schedule is the cyclical schedule as determined above, but with less fixed capacity and the clinic may add an extra block each week. The optimal policy for determining whether or not to schedule an additional block is determined by means of a Markov decision process.

The state of the Markov process is the number of patients in the queue for all patient types: $\mathbf{s}=\left[q_{1}, \ldots, q_{N}\right] \in \mathcal{S}$, with $N$ the number of patient types, and $q_{p}$ is finite for all $p$. Transitions and decisions occur at the end of every cycle. Each decision epoch, the clinic either schedules an additional block at the end of the next cycle $(a=1)$, or not $(a=0)$. The additional block is the same each time it is added to the schedule, and is obtained with the MIP. Let $P\left(q_{p}^{\prime} \mid q_{p}, a\right)$ denote the probability that the queue length of type $p$ equals $q_{p}^{\prime}$ at the next decision epoch, given that the current queue length is $q_{p}$ and decision $a$ is taken. Furthermore, let $Y^{t}$ denote the total number of blocks scheduled by the MIP in one cycle, and $\alpha_{p}$ the number of appointment slots per block for type $p$ patients. 
Then, given that each patient type can only be assigned to appointment slots that are reserved for their type, the transition probabilities are:

$$
P\left(\mathbf{s}^{\prime} \mid \mathbf{s}, a\right)=P\left(q_{1}^{\prime} \mid q_{1}, a\right) \cdot \ldots \cdot P\left(q_{N}^{\prime} \mid q_{N}, a\right),
$$

with

$$
\begin{aligned}
& P\left(q_{p}^{\prime} \mid q_{p}, a\right)= \\
& \begin{cases}P\left(A_{p}=q_{p}^{\prime}\right) & \text { if } q_{p} \leq t_{p i}, \\
P\left(A_{p}=q_{p}^{\prime}\right) \sum_{i=0}^{j-1} P\left(c_{p}^{t}=t_{p i}\right) & \text { if } t_{p j} \leq q_{p} \leq t_{p, j-1}, \\
\quad+\sum_{i=j}^{Y^{t}} P\left(A_{p}=q_{p}^{\prime}-q_{p}+t_{p i}+a \cdot \alpha_{p}\right) P\left(c_{p}^{t}=t_{p i}\right) & \text { for } j=1, \ldots, Y^{t}, \\
\sum_{i=0}^{Y^{t}} P\left(A_{p}=q_{p}^{\prime}-q_{p}+t_{p i}+a \cdot \alpha_{p}\right) P\left(c_{p}^{t}=t_{p i}\right) & \text { else. }\end{cases}
\end{aligned}
$$

Here, $A_{p}$ is the random number of arrivals for patient type $p$ each cycle, and $t_{p i}$ the capacity for patients of type $p$ in case $i$ blocks are canceled. We assume that each block is canceled with probability $u$, so $P\left(c_{p}^{t}=t_{p i}\right)$ follows a binomial distribution with probability $u$.

To enhance readability, we assume that the cycle length equals the access time norm, but the formulas can be adapted to different values. Costs are incurred when there are idle time slots or when the access time exceeds one cycle length. We use the expected number of empty slots and the expected queue length at the end of the cycle for the direct costs, resulting in:

$$
\begin{gathered}
C(\mathbf{s}, a)=\sum_{i=0}^{Y^{t}}\left[P ( c _ { p } ^ { t } = t _ { p i } ) \cdot \left(C^{a} \sum_{p=1}^{N}\left(q_{p}+\mathbb{E}\left[A_{p}\right]-t_{p i}+a \cdot \alpha_{p}\right)^{+}\right.\right. \\
\left.\left.+C^{e} \sum_{p=1}^{N}\left(t_{p i}+a \cdot \alpha_{p}-q_{p}-\mathbb{E}\left[A_{p}\right]\right)^{+}\right)\right]
\end{gathered}
$$

The total discounted value of the MDP, given policy pol, satisfies the Bellmanequations:

$$
V_{p o l}(\mathbf{s})=\max _{a}\left\{C(s, a)+\beta \sum_{\mathbf{s}^{\prime} \in S} P\left(\mathbf{s} \mid \mathbf{s}^{\prime}, a\right) V_{p o l}\left(\mathbf{s}^{\prime}\right)\right\},
$$

with $\beta$ the discount factor. We assume $\beta=0.95$ to reflect that future access times are important to take into account with the current decision making.

Opposed to the queueing model, the MDP cannot be evaluated for each patient type independently, as we may only add an entire block and blocks consist of appointment slots for all patient types. In order to both avoid state space explosion and increase the practical relevance of the policy, we aggregate all patient types in the MDP model. As the purpose of the MDP is only to determine whether or not to add a block and the actual scheduling is carried out following the results of Section 9.2.2, the aggregated form used here is justified. Note that we could also assume patients arrive and are treated in batches in order to reduce the state space, but this would result in a more difficult and therefore less practical policy. 
Table 9.1 Weekly demand and capacity for the case study.

\begin{tabular}{cccc}
\hline Type $p$ & $L_{p}$ & $\begin{array}{c}\mathbb{E}\left[A_{p}\right] \\
\text { (patients) }\end{array}$ & $\begin{array}{c}\text { MIP capacity } \\
\text { (appointment slots) }\end{array}$ \\
\hline 1 & 2 & 7.4 & 9 \\
2 & 2 & 115.9 & 130 \\
3 & 2 & 14.0 & 17 \\
4 & 2 & 29.4 & 34 \\
5 & 2 & 8.3 & 11 \\
6 & 2 & 27.7 & 33 \\
7 & 2 & 5.7 & 8 \\
8 & 1 & 24.7 & 28 \\
\hline
\end{tabular}

\subsection{Application}

In this section we assess the quality of the schedules derived by the MIP and MDP approaches by means of the case study of the surgical outpatient clinic of the JBH, for the sub-specialty oncology. To this end, we conducted a simulation study and compared the different appointment schedules on the following performance measures: average access time, the probability that the access time exceeds one week, and the utilization of the appointment slots. The JBH aims for an access time norm of one week for as many patients as possible, which is a tighter norm than the national norm.

As in many hospitals, in the JBH it is not possible to use data on the times appointments were requested, as only the realized appointment times are registered. This also implies that we cannot incorporate appointments scheduled for patients who did not show up, and cannot derive the arrival distribution from the data. Therefore, as input for all models we use the data in Table 9.1, which is based on the realized appointment schedules instead of the times the appointments are requested. Additionally, we assume that the arrival distribution is Poisson with a constant rate.

In the discrete event simulation patients of different types arrive one by one according to a stochastic process and take the first available appointment slot that is reserved for their type. Each block has a certain probability of being canceled, which is varied in the simulation.

We solve the MIP using AIMMS ${ }^{\circledR}$ and use the policy iteration algorithm [405], implemented and solved in Matlab ${ }^{\circledR}$ to obtain the optimal policy for the MDP. The simulation is programmed in $\mathrm{C}++$. We use common random numbers [295] for a fair comparison between the schedules. We simulate 200 runs with 260 clinic days each, in order to obtain minimally $5 \%$ relative precision.

The results for the MIP in this section are obtained for $C^{a}=C^{e}$, e.g., equal weights for access and idle time. We assume that the probability a block is canceled is independent for each block and equals $10 \%$, so $u=0.1$, which is desired by the JBH but currently the cancellation probability is slightly higher. All schedules are obtained using this assumption, and with simulation we investigate the effect when the actual cancellation probability is different. Next to the MIP 
Figure 9.1 Results static and dynamic scheduling for multiple cancellation probabilities.

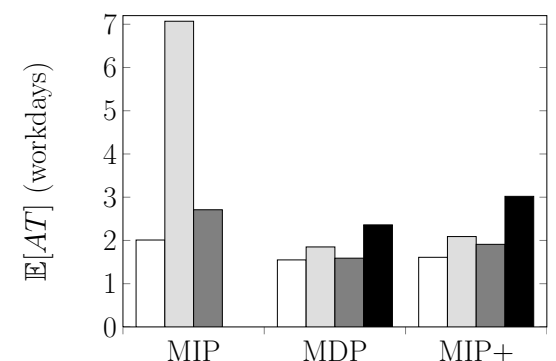

(a) Expected access time

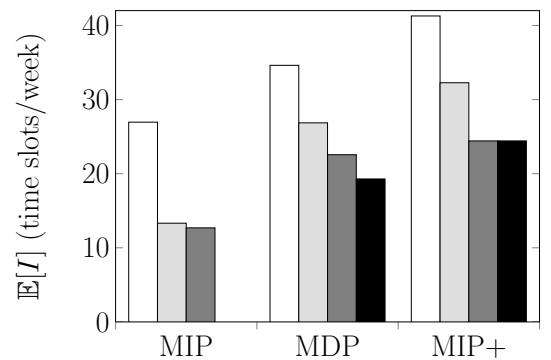

(b) Expected idle time

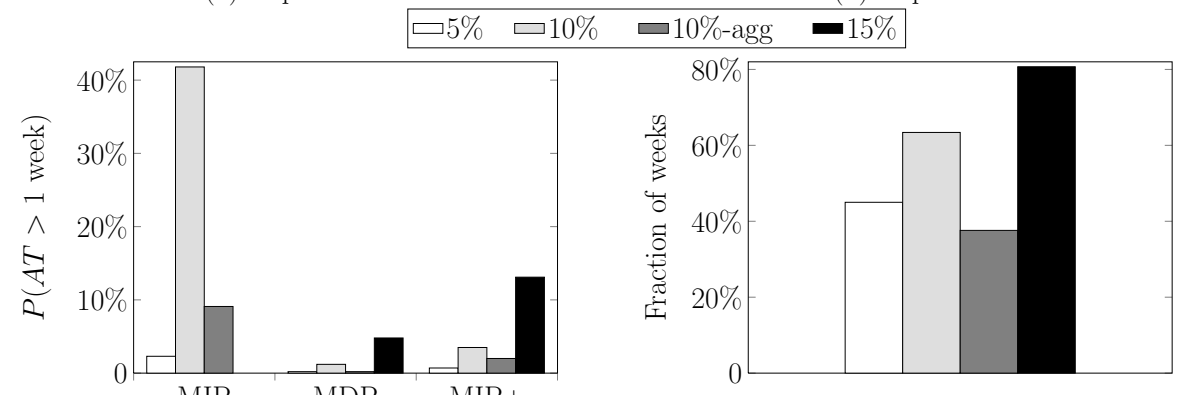

(c) Prob. of exceeding $A T$ norm

(d) Weeks with added block by MDP

and MDP schedule, we investigate scenarios in which the capacity is pooled, i.e., aggregated for all patient types, denoted by suffix '-agg'. The MDP schedule adds capacity to the MIP schedule in a significant number of weeks. In order assess the added value of dynamic scheduling compared to static scheduling we additionally investigate a MIP schedule with the same total capacity as the MDP schedule, which is labeled 'MIP+'; the additional capacity is added by adding (part of) a block to the MIP schedule each week such that the total capacity equals the capacity of the MDP schedule over the entire simulation run.

The schedule created by the MIP for this scenario requires 512 time slots in 15 blocks each week, divided over $7 \times 18$ appointment slots in the morning and $8 \times 18$ appointment slots in the afternoon. Note that most patient types require two time slots per appointment slot, and we can schedule more than ten blocks per week (five working days $\times$ two blocks per day) because multiple surgeons can work in parallel. Figure 9.1 depicts simulation results for different cancellation probabilities, performance measures and scheduling policies. Recall that all schedules in this figure are obtained assuming that the cancellation probability is $10 \%$ and the cancellation probability is only varied in the simulation. The simulated scenario with $15 \%$ cancellation probability has insufficient capacity to schedule all arriving patients and is therefore not depicted in Figure 9.1.

As expected, from the simulation results it appears that the average access time and access time norm compliance improve significantly in case of a lower 
Table 9.2 MDP policy for different $C^{a}$ and $C^{e}$.

\begin{tabular}{cccc}
\hline $\begin{array}{c}C^{a} \rightarrow \\
C^{e} \downarrow\end{array}$ & 1 & 2 & 5 \\
\hline 1 & 22 & 20 & 1 \\
2 & 32 & 22 & 9 \\
5 & 41 & 35 & 22 \\
\hline
\end{tabular}

cancellation probability, more capacity (MDP and MIP + schedules), and when all capacity is pooled. With the current capacity and $10 \%$ cancellations $38.3 \%$ of the patients cannot be scheduled within the norm, see Figure 9.1 (c). In the other schedules there also are patients for which the access times exceed the norm, but these numbers of patients are acceptable for the clinic.

The MDP and MIP + schedules have relatively many idle slots each week, the $\mathrm{MIP}+$ schedule slightly more than the MDP schedule. As each block consists of 34 time slots, on average one block is canceled each week, which is acceptable for the JBH; because the appointments are planned in advance the surgeons can use this time to schedule other activities. Figure 9.1 (d) depicts how often the MDP policy prescribes to schedule an additional block; this policy implies that for the $10 \%$-scenario on average $2.5 \%$ capacity is added to the schedule.

From Figures 9.1 (a) and (c) it appears that the MDP policy outperforms the MIP + policy with respect to the access time, i.e., dynamic scheduling outperforms static scheduling. This is an intuitive result, as both schedules contain the exact same capacity, but the dynamic schedule schedules the capacity only at times with many patients in the system. We conjecture that dynamic scheduling outperforms static scheduling for all realistic instances. However, in an artificial instance in which alternately $Y^{t}<x<2 Y^{t}$ patients and zero patients arrive each week, the MIP + schedule will outperform the MDP schedule; the MDP policy will add capacity in quiet weeks and will not add capacity in busy weeks, thereby increasing both the access times and the number of idle slots.

Similar to the MIP, Figure 9.1 only depicts the results of the MDP with equal costs for access and idle time. With these parameters, it is optimal that an extra block is scheduled when the total queue length exceeds 22 patients. We investigated the effect of other values of $C^{a}$ and $C^{e}$, see Table 9.2. As expected, the threshold to schedule an additional block decreases for relatively large access time costs, and vice versa. As the results of these schedules are obvious, they are omitted in this chapter. Additionally, we investigated policies that depend on all patient types separately, but we do not present those results here as they are more complex and did not improve the results significantly. From a practical perspective, more complicated policies are of little use for the JBH.

In Figure 9.2 we depict simulation results of the conditional average access time of patients arriving in week $x$ for several scheduling policies with $10 \%$ cancallation probability. We conclude that for this case study in most weeks the conditional average access time of a patient is shorter than 5 days, but the static schedule results in several time periods where the norm is exceeded. The condi- 
Figure 9.2 Week-dependent results for $10 \%$ cancellation.

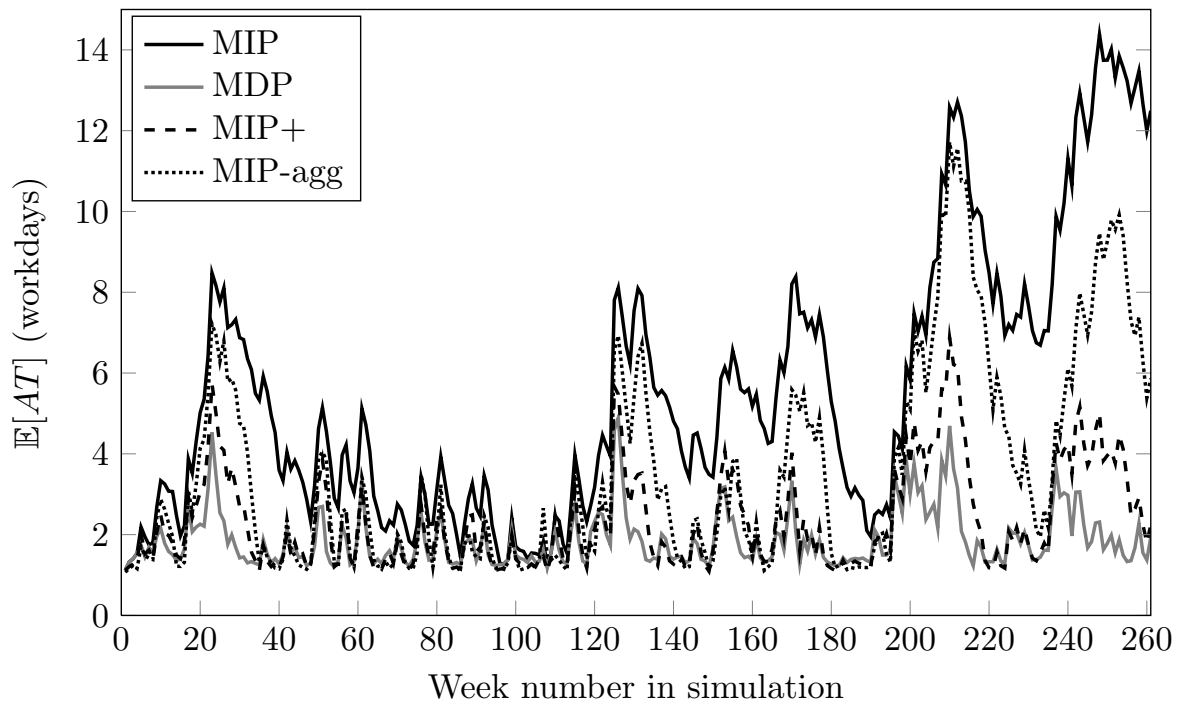

tional performance is similar in the scenarios with different cancellation probability.

In reality, patients could have a preference for one of the doctors when making an appointment. With the simulation model, we investigated scenarios in which $40 \%$ or $80 \%$ of the patients had a preference for a random doctor; the JBH does not have data on patient preferences. These preferences, obviously, increased the average access time and number of idle slots. The probability that the access time exceeds the norm approximately doubled compared to the scenarios where patients do not have preferences. These effects should be taken into account when the results of the models would be implemented in practice, and imply that the performance as presented here will probably not be achieved by the JBH.

For the performance of the clinic it is important to minimize the cancellation probability, as the simulation results show this probability significantly affects all performance measures when the load of the system is close to one. For the MDP and MIP + schedules, the effect of different cancellation probabilities is relatively small. Pooling all patient types would significantly increase the clinic's performance, even resulting in better performance compared to the scenario with only $5 \%$ cancellation probability.

\subsection{Discussion}

In this chapter we have presented an SMIP and two approximation methods by which an optimal static and dynamic appointment schedule can be made for an outpatient clinic with time-varying demand and capacity. We have shown that optimally allocating flexible capacity increases both the efficiency and per- 
formance of the hospital, thus improving the quality of care. Although this methodology is designed for a hospital case study, it can also be applied to different appointment systems with time-varying demand and/or capacity.

The JBH is currently discussing the implications of this research, and studying the implementation possibilities. The main discussion is on the addition of $2.5 \%$ capacity and the flexible scheduling policy. The required additional capacity is currently not available in theory, but is presumably currently available because surgeons work overtime. Pooling capacity does not seem a valid option, as there are patient types with shorter access time norms that arrive relatively less often. Scheduling an additional block for the next week also seems too short notice, so future work could be extending the models to make longer-term decisions.

The models presented here may be enhanced to take into account more practical settings: patients that do not take the first available slot; or patients that are assigned to a slot that is not reserved for their own type, e.g., when slots for a different type are still idle on short notice. Additionally, scenarios that allow surgeons to work overtime up to a certain maximum per block or week would better reflect the current practice at the $\mathrm{JBH}$ and most outpatient clinics.

The results of our research might deviate from reality due to the assumption of Poisson arrivals. Additional interesting future work could be to measure the arrival process in reality, and use that as input for our model. All models used in this study can be extended to include time-dependent (non-Poisson) arrival distributions.

In reality, temporarily adding capacity is usually done after a period with less available capacity, e.g., after holidays. It would be interesting to investigate if this policy is effective, or that the hospital could better use the flexible schedule throughout the year. For a realistic comparison, one should also simulate the flexible schedule including patients' preferences and doctors taking weeks off.

\subsection{Appendix}

For completeness, we provide the formulas relating the daily capacity to the expected access time and number of empty slots through the stationary distribution of $B_{d p}$, the backlog for patient type $p$ on day $d$. The discrete time queueing model applied in this chapter is already introduced in Appendix 6.7. Recall that each day $d$ a random number of type $p$ patients, $A_{d p}$, arrives and a maximum number of patients $c_{d p}$ can be seen. Additionally, the conversion from $c_{p}^{t}$ to $c_{d p}$ is $c_{d p}=\left\lfloor c_{p}^{t} / D\right\rfloor$, with $\lfloor x\rfloor$ denoting rounding down to the nearest integer, and the remaining $c_{p}^{t}-D \cdot\left\lfloor c_{p}^{t} / D\right\rfloor$ days added to days with the lowest index and enough surgeons available. Furthermore, recall that $B_{d p}=\left(B_{d-1, p}-c_{d p}\right)^{+}+A_{d p}$ and the transition probabilities for this model are given by:

$$
P\left(B_{d p}=q^{\prime} \mid B_{d-1, p}=q\right)=P\left(A_{d p}=q^{\prime}-\left(q-c_{d p}\right)^{+}\right) .
$$

We consider a cyclic schedule, so index $d:=d \bmod D$, with $D$ the cycle length. As the JBH does not have data on the arrival distribution, we assume 
Poisson arrivals, which implies:

$$
P\left(A_{d p}=j\right)=\frac{\lambda_{d p}^{j} e^{\lambda_{d p}}}{j !},
$$

with $\lambda_{d p}$ the arrival rate on day $d$. The stationary distribution of $B_{d p}$ is obtained by solving $\pi \mathbf{P}=\pi$, with $\mathbf{P}$ the transition probability matrix. We require the expected access and idle time, which are derived as follows.

Recall that $\pi_{d p i}$ denotes the stationary probability that the backlog on day $d$ equals $i$ for type $p$ patients. The expected number of empty slots per cycle is then given by:

$$
\mathbb{E}\left[I_{p}\right]=\sum_{d=0}^{D-1} \sum_{q=0}^{c_{d p}-1}\left(c_{d p}-q\right) \pi_{d p q}
$$

The expected access time is derived by conditioning on the backlog of patients on a day $d$ :

$$
\mathbb{E}\left[A T_{p}\right]=\sum_{d=0}^{D-1} \sum_{y=1}^{\infty} P\left(A T_{d p}>y \mid B_{d p}=q\right) \pi_{d p q} \cdot \frac{\mathbb{E}\left[A_{d p}\right]}{\sum_{j=0}^{D-1} \mathbb{E}\left[A_{j p}\right]} .
$$

Here, $P\left(A T_{d p}>y \mid B_{d p}=q\right)$ is the probability that the access time of a type $p$ patient arriving on day $d$ exceeds $y$ days, given that the backlog at the end of day $d$ equals $q$. Recall that $c_{d p}(y)=\sum_{i=1}^{y} c_{d+i, p}$ denotes the sum of the capacity from day $d+1$ until day $y, P\left(A T_{d p}>y \mid B_{d p}=q\right)=1$ if $c_{d p}(y) \leq q$, and for $c_{d p}(y)>q$ it holds:

$$
P\left(A T_{d p}>y \mid B_{d p}=q\right)=\frac{\sum_{j=c_{d p}(y)+1}^{\infty}\left(j-c_{d p}(y)\right) P\left(A_{d p}=j\right)}{\mathbb{E}\left[A_{d p}\right]} .
$$

Concluding, the average access time is given by:

$$
\mathbb{E}\left[A T_{p}\right]=\sum_{d=0}^{D-1} \sum_{y=1}^{\infty} \frac{\sum_{j=c_{d p}(y)+1}^{\infty}\left(j-c_{d p}(y)\right) P\left(A_{d p}=j\right)}{\sum_{j=0}^{D-1} \mathbb{E}\left[A_{j p}\right]} \cdot \pi_{d p q} .
$$

Formula $f^{e}\left(c_{p}^{t} \mid \lambda_{p}, u\right)$ equals equation (9.12), for which we need to derive $c_{d p}$ from $c_{p}^{t}$ as explained in Section 9.2.1 and above. In order to evaluate formula $f^{a}\left(c_{p}^{t} \mid \lambda_{p}, u\right)$, we determine $c_{d p}(y)$ with $y$ equal to five days in our case study, and evaluate equation (9.13). 



\section{Part V}

\section{Optimizing throughput at the ED}

Chapter 10

N.M. van de Vrugt and R.J. Boucherie. Assigning treatment rooms at the Emergency Department. Operations Research for Health Care, 8:62-70, 2016.

Chapter 11

N.M. van de Vrugt and I. Ziedins. Sequentially assigning and prioritizing patients at Emergency Departments. Working paper. 



\section{0 department}

Assigning treatment rooms at the emergency

\subsection{Introduction}

Emergency department (ED) overcrowding has many negative effects, for both patients and ED staff. ED overcrowding leads for example to an increase in the number of medical errors, long waiting times and high levels of stress for ED staff (see for example $[234,388,517]$ ). Therefore, governments have set legal norms on the waiting time and length of stay $(\mathrm{LoS})$ of patients visiting the ED. Meeting these norms is often difficult due to an increasing demand for acute care and the closure of many EDs and hospital beds [274].

The waiting time and LoS of ED patients is influenced by many factors, among others the patient's urgency class and type. In the Netherlands, patients are both triaged and assigned to a specialty upon arrival at the ED. The triage category of a patient determines the order in which patients are treated. Treatment can start when there is a treatment room and the patient's doctor is available. The specialties with the highest number of patients are represented at the ED at all times, either by a specialist or a resident (together with physicians collectively called 'doctors' in this chapter). Other specialties have to be called for assistance, implying that patients at least have to wait for the travel time of the doctor.

Typically at an ED, one doctor occupies multiple rooms in parallel; when a patient requires diagnostic tests, the doctor visits other patients. Each specialty/doctor has a different working routine: emergency physicians are for example specially trained for only providing urgent care, while other doctors tend to provide care more like they are used to outside the ED [274]. As a consequence, LoSs and waiting times of different patient types differ significantly, and the room utilization can be unbalanced over the doctor types. The unbalanced room utilization possibly also affects patients' LoS and waiting time.

Besides the LoS and waiting time, the probability that the ED is 'full' (blocking probability) is an important performance measure. When the number of patients at the ED exceeds a certain level, the ED staff will call in an extra doctor and/or defer new patients. The first will result in additional costs, while the latter negatively affects the image of the hospital.

In this chapter we investigate several room assignment policies and doctor 
working routines at an ED and determine the Pareto-efficient combinations with respect to the performance measures mentioned above. To this end, we invoke a continuous time queueing model in which patients may require diagnostic tests, implying that doctors visit their patients a random number of times and interact in sharing the available treatment rooms. We investigate a case study of the JBH and several additional illustrative cases. By means of a discrete event simulation, we investigate how the obtained policies perform in a more realistic setting.

We also study the LoS of an accepted patient conditioned on the state found in the ED upon arrival. This conditional waiting time and LoS information may be provided to the patients to inform them about their expected length of stay at the ED, which adds to patient satisfaction [57].

In the next section we will provide an overview of related literature. The model is described in Section 10.3. Section 10.4 provides the analytical results, Section 10.5 the numerical results, and Section 10.6 the conclusion and discussion.

\subsection{Related literature}

The literature that is related to this chapter consists of papers on EDs and of queueing models that are similar to the one considered in this chapter. Both types of literature will be summarized below.

EDs are modeled most often by means of queueing theory and computer simulation. Typical focus areas of these works are capacity dimensioning and patient routing, with the objectives to minimize patient waiting time, maximize patient throughput or increase staff utilization [242]. In patient routing the effects of for example parallelization of tasks, a 'fast-track' system, and letting patients wait outside the treatment room are studied.

There are many papers that apply queueing models to an ED, but only a few papers are closely related to this chapter as they consider patients who are visited more than once by the doctor, see for example [158, 246, 532]. These studies consider a multi-server queueing network in which customers can re-enter a queue, and the authors aim to determine appropriate staffing levels to achieve certain performance targets. Differences between the models in these references (and the references therein) and the model considered here, are that patients do not return to their own doctor and that it is assumed that treatment can start once the doctor is available. This implies that either there always is a sufficient number of treatment rooms, or patients await their test results outside the room.

In a recent review, Wiler et al. [517] state that most queueing approaches to modeling an ED assumed that: (1) arrival rates are constant, (2) patients do not deteriorate, and (3) patients are not seen by same doctor again. Here we also assume (1) and (2), but allow for patients to be seen by the same doctor more than once. For more literature on modeling EDs, the reader is referred to recent literature reviews $[242,388,517]$ and the references therein.

A different but related topic is the optimal assignment of servers to two types of calls in a callcenter, studied by for example Bhulai and Koole [46]. Here the 
question is when to assign servers to outbound calls in such a way that inbound calls still achieve high service levels. Differences with our work are that in [46] each call visits the server only once, and each server has its own line (opposed to doctors sharing multiple treatment rooms).

Our contribution to both fields of literature is that we incorporate the ordering of diagnostic tests and doctors revisiting their patients in a queueing model for an ED. We consider a model with two resource types (doctors and rooms) in which a customer can only get service when both resource types are available at the same time. To the best of our knowledge, this type of model has not been analyzed before. This model is applied to an ED, but could easily be adapted to, for example, an outpatient clinic in which multiple doctors share the available treatment rooms.

The method used in this chapter has been applied before to telecommunication systems, see for the most related papers [56, 313, 314, 410]. These systems typically have two call types: inelastic (speech) calls, and elastic (data) calls. The inelastic calls require one unit of capacity (bandwidth) throughout their entire service time. The elastic calls share the remaining capacity in a processor sharing fashion. Important performance measures are the (average) throughput, sojourn time and blocking probability. At an ED, multiple types of patients also have to share the available capacity (treatment rooms). Although the processor sharing service discipline is not applicable for an ED, it will be shown that the methods applied to telecommunication systems can still be applied.

\subsection{The Model}

In this chapter, we focus on a system with two doctors, since in many Dutch hospitals such as the JBH most of the non-urgent patients require a surgeon or an internist and both specialties are represented at the ED at all times. Patients that require other specialties are either treated by an emergency physician (working at the ED from 7:00 to 23:00) or by a specialist doctor who is called for assistance.

Upon arrival at the ED, patients are triaged, assigned to a doctor, and join a waiting area. We only consider patients that are triaged as non-urgent, and are therefore treated on a first come, first served basis. The triage is typically not the bottleneck at an ED, and therefore we only focus on the process after triage.

To model this ED in which two doctors share multiple treatment rooms to treat two types of patients with equal priority and each type of patient has its own queue and doctor, we consider a queueing system as depicted in Figure 10.1. There are more treatment rooms than doctors, depicted by squares and circles respectively. The number of patients in the system is finite for both patient types and patients are assumed to arrive at their assigned queue according to a random process with constant arrival rate (we numerically investigate this assumption in Section 10.5). When the required doctor is inactive upon arrival of a patient and a treatment room is available, the treatment commences immediately. Otherwise, the patient is either blocked (if the maximum number of patients of this type is 
Figure 10.1 Schematic representation of the queueing network.

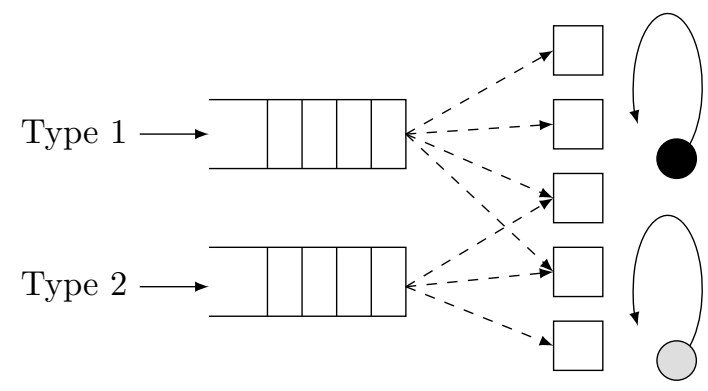

reached) or placed in a waiting area.

The treatment of a patient consists of at least one visit by the doctor; between two visits of the doctor, patients take diagnostic tests. At the instant the treatment of a patient begins, he is assigned a treatment room. This room will be assigned to this patient during the entire treatment time, even if the patient is not always physically in the room, which is the common policy at Dutch hospitals. We call each time a doctor visits a patient a 'phase' and the 'consultation time' of a patient denotes the total time a doctor has to spend with this patient in a room (so the sum of all phases). The treatment time thus includes the consultation time and possibly time between two doctor visits and time for taking diagnostic tests. To enhance tractability, we assume that both types of doctors visit their patients in random order. The LoS equals the waiting time before a room is assigned plus the treatment time.

During each visit the doctor can decide that the patient requires (further) diagnostic tests. Therefore, after each phase completion a patient stays in the system with a certain probability. When a patient's phase is completed but this patient does not leave the ED, the doctor can consult either a patient from the waiting area ('new patient') or in another treatment room ('existing patient'). The first option requires an extra room. The decision which of the two options to choose, depends on the working routine of the doctor and will be varied. If at phase completion the patient cannot leave the system and there is no other patient in the system, the doctor will become 'inactive', for example perform administrative tasks, for a random time. If a new patient arrives during an inactive period and there is a room available, the new patient is consulted immediately.

An interesting feature of this model is that in order to be treated, each patient requires two resources at the same time: a room and a doctor. Rooms 'serve' patients in a first come first serve fashion, while doctors visit their patients in random order. A patient occupies a room throughout its entire treatment time, while the doctor will only be in the room with the patient occasionally. Without the doctor present in the room, the treatment of the patient is 'paused'.

The system under consideration can be modeled as a continuous time Markov 
process with two types of patients and one doctor for each patient type. This system has two independent Poisson arrival streams with rates $\lambda_{1}$ and $\lambda_{2}$. The state of the system is denoted by $\mathbf{n}=\left(n_{1}, r_{1}, i_{1} ; n_{2}, r_{2}, i_{2}\right)$, with: $n_{j}$ the number of patients in system; $r_{j}$ the number of rooms occupied; $i_{j} \in\{0,1\}$ an indicator that equals 1 if the doctor is with a patient and 0 otherwise; and $j=1,2$ denotes the different patient and doctor types. The indicator is used when there is a phase completion of a patient that is the only patient present of his type. At such phase completions, the doctor will be inactive until either the patient returns from his tests, or a new patient of his type arrives. The time a doctor is inactive is therefore the minimum of two exponential times, with parameters $\nu_{j}$ and $\lambda_{j}$ respectively.

Each phase of consultation time is exponentially distributed with rate $\mu_{j}$ for type $j$. Define $p_{j}$ the probability that the patient's consultation time ends after completion of a phase. Then, the consultation time consists of a geometric number of exponential phases, and thus it is readily derived that its probability mass function is given by $f_{j}(x)=p_{j} \mu_{j} \exp \left(-p_{j} \mu_{j} x\right)$. Furthermore, let $M_{j}$ be the maximum number of patients in the system for type $j$. The generator $\mathbf{Q}$ for this process is developed in Appendix 10.7.

\subsection{Analytical results}

Since for both patient types the analysis is the same, we will omit most patient type subscripts in the development which follows, using the subscript $j$ to indicate one of the types where necessary, and refer to 'patient' instead of mentioning the types. The analysis follows the lines of $[313,410]$.

\subsubsection{Basic performance measures}

We obtain the stationary distribution $\pi$ of this system by solving $\pi \mathbf{Q}=0$. Using $\pi$, we can obtain average performance measures like the expected number of patients in the queue $\left(N^{q}\right)$ and system $\left(N^{s}\right)$, and the blocking probability $P^{b}$ for both patient types:

$$
N_{j}^{q}=\sum_{\mathbf{n} \mid n_{j} \geq r_{j}}\left(n_{j}-r_{j}\right) \cdot \pi_{\mathbf{n}}, \quad N_{j}^{s}=\sum_{\mathbf{n}} n_{j} \cdot \pi_{\mathbf{n}}, \quad P_{j}^{b}=\sum_{\mathbf{n} \mid n_{j}=M_{j}} \pi_{\mathbf{n}} .
$$

Using Little's formula, we can obtain the expected waiting time $W_{j}$ and LoS $S T_{j}$ of an accepted patient by:

$$
W_{j}=\frac{N_{j}^{q}}{\lambda_{j}\left(1-P_{j}^{b}\right)}, \quad S T_{j}=\frac{N_{j}^{s}}{\lambda_{j}\left(1-P_{j}^{b}\right)} .
$$

\subsubsection{The conditional expected LoS}

The LoS of a patient consists of two stages: the time from triage until entering the treatment room (waiting time) and the treatment time. We determine the 
conditional expected LoS by tagging an arriving patient and running the Markov chain from the state the patient encountered upon arrival until the patient leaves the system. The conditional expected waiting time $\alpha_{\mathbf{n} j}$ depends on the number of patients in the system upon arrival of the tagged patient. The treatment time of the tagged patient depends both on his consultation time, and the (possibly varying) number of rooms his doctor uses during his treatment time. The conditional expected treatment time $\tau_{\mathbf{n}^{\prime} j}(x)$ of the tagged patient is conditioned on his consultation time $x$ and the state of the system in which the treatment of this patient commences. This state is not necessarily the state which the tagged patient encountered upon arrival. Therefore, we also need to determine the conditional transition probabilities $\psi_{j}\left(\mathbf{n}, \mathbf{n}^{\prime}\right)$ : the probability that a patient arriving in state $\mathbf{n}$ commences his service in state $\mathbf{n}^{\prime}$. The expected LoS of an admitted patient, conditioned on the state of the system at the instant the patient has arrived, is then expressed by:

$$
S T_{\mathbf{n} j}=\int_{0}^{\infty} \sigma_{\mathbf{n} j}(x) f_{j}(x) \mathrm{d} x, \text { with } \sigma_{\mathbf{n} j}(x)=\alpha_{\mathbf{n} j}+\sum_{\mathbf{n}^{\prime}} \tau_{\mathbf{n}^{\prime} j}(x) \psi_{j}\left(\mathbf{n}, \mathbf{n}^{\prime}\right) .
$$

Here, $\sigma_{\mathbf{n}, j}(x)$ denotes the conditional LoS of a patient of type $j$ arriving in state $\mathbf{n}$ (including the new patient) with consultation time $x$. Note that the LoS does not exist for certain states, for example states with $n_{j}=0$. From the conditional expected LoS, the expected LoS can be obtained by:

$$
S T_{j}=\sum_{\mathbf{n} \mid n_{j}>0} \frac{\pi_{\mathbf{n}-\mathbf{e}_{j}}}{\sum_{\mathbf{n} \mid n_{j}>0} \pi_{\mathbf{n}-\mathbf{e}_{j}}} S T_{\mathbf{n} j}=\sum_{\mathbf{n} \mid n_{j}>0} \frac{\pi_{\mathbf{n}-\mathbf{e}_{j}}}{1-P_{j}^{b}} \cdot S T_{\mathbf{n} j},
$$

with $\mathbf{n}-\mathbf{e}_{j}$ denoting a system with one patient of type $j$ less. Equation (10.3) consists of multiple parts that can be obtained by the steps summarized in Appendix 10.8, and in more detail in $[314,410]$. We implemented equation (10.3) and all calculation steps in Matlab ${ }^{\circledR}$ in order to perform a numerical study.

\subsection{Numerical results}

In this section we first describe the JBH case study and its results. Hereafter, we provide numerical results for several illustrative cases. The policies we investigate for this queueing model are all of threshold-type: for both specialties we define a maximum number of rooms $\delta_{j}$ that specialty $j$ can use in parallel. The room assignment policies we investigate only include policies in which all rooms could theoretically be used; for example for a case with five rooms available the policy $\left(\delta_{1}, \delta_{2}\right)=(3,1)$ was not evaluated since the maximum number of rooms in use is four here. We further distinguish two different working routines per doctor: either he visits a new patient first at phase completion (called NF), or he returns to an existing patient first (called EF). The working routines are therefore denoted by: EF-EF, EF-NF, NF-EF and NF-NF (in which the working routine of doctor 
1 is mentioned first). For all performance measures, we determine the Pareto efficient 'working routine + room assignment policy' combinations (RPCs), which we denote by (working routine doctor $1, \delta_{1}$, working routine doctor $2, \delta_{2}$ ).

In addition to the analytical results, we conduct a discrete event simulation study to investigate the policies in a more realistic setting: the arrival rates are time-dependent and obtained from data of the JBH, see Figure 10.2. We

Figure 10.2 Time-dependent arrival rates obtained from JBH data.

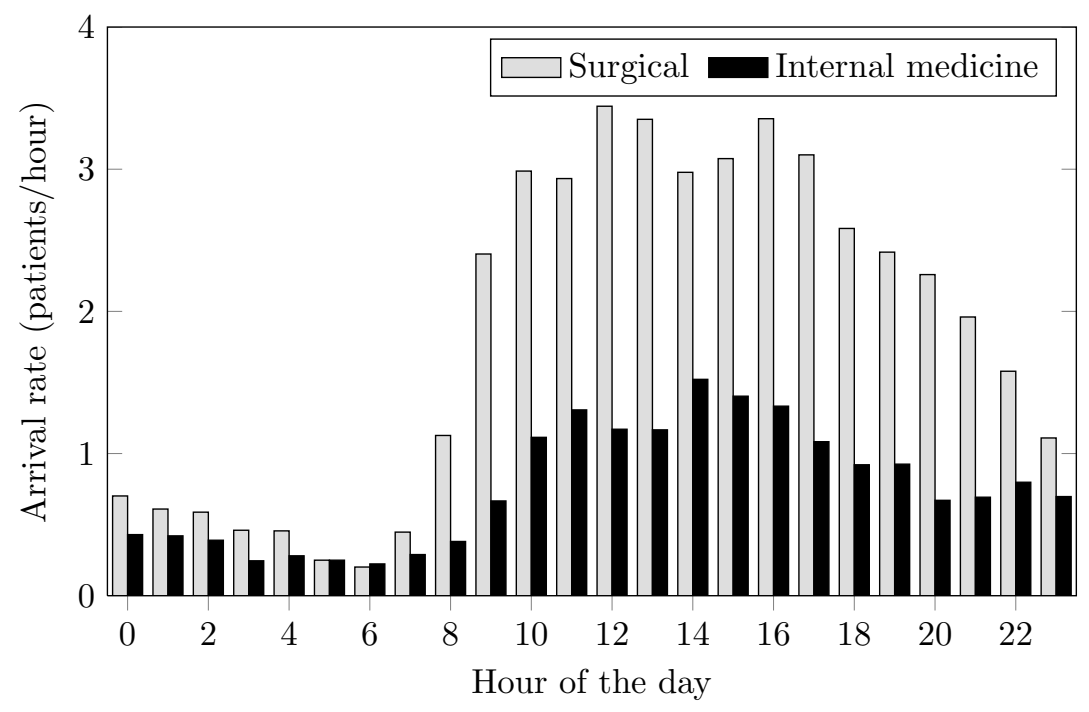

simulate 5000 days for 20 runs such that the relative precision of the waiting time and LoS is always below $1 \%$. We use common random numbers [294].

\subsubsection{JBH case study}

Data We only consider surgical (type 1) and internal medicine (type 2) patients in our case study, which represents $66 \%$ of the non-urgent ED-patients at the JBH. Typically at an ED, arrival rates are not constant during the day. The problem of dividing rooms among the doctors is most prevalent during the peak hours. Therefore, we take the average arrival rate between 10:00 and 17:00 as input for the model, and refer to this rate as the 'peak arrival rate'. All input parameters are obtained from JBH data or estimated when not available, and are displayed in Table 10.1.

In the current working routine of the JBH, there is no policy for the assignment of rooms and both doctors visit new patients first. There are eight available treatment rooms at the ED. In our study, since we only consider $66 \%$ of the patients, we assume there are only five treatment rooms. For five treatment rooms, there exist 19 allowed room assignment policies: $(5,5),(5,4),(5,3),(5,2),(5,1)$, $(4,5),(4,4),(4,3),(4,2),(4,1),(3,5),(3,4),(3,3),(3,2),(2,5),(2,4),(2,3),(1,5)$, 
Table 10.1 Input parameters for the JBH case study.

\begin{tabular}{lccccc}
\hline & $\lambda\left(h^{-1}\right)$ & $\mu\left(h^{-1}\right)$ & $\nu\left(h^{-1}\right)$ & $p$ & $M$ \\
\hline Surgical & 3.15 & $8.57^{*}$ & $1.93^{*}$ & $0.45^{*}$ & 15 \\
Internal medicine & 1.26 & 7.09 & 1.93 & $0.30^{*}$ & 10 \\
\hline * = parameter is estimated & & & &
\end{tabular}

Figure 10.3 Analytical (left) and simulated (right) waiting time for different policies for the JBH case study, non-dominated marked gray, efficient marked black.
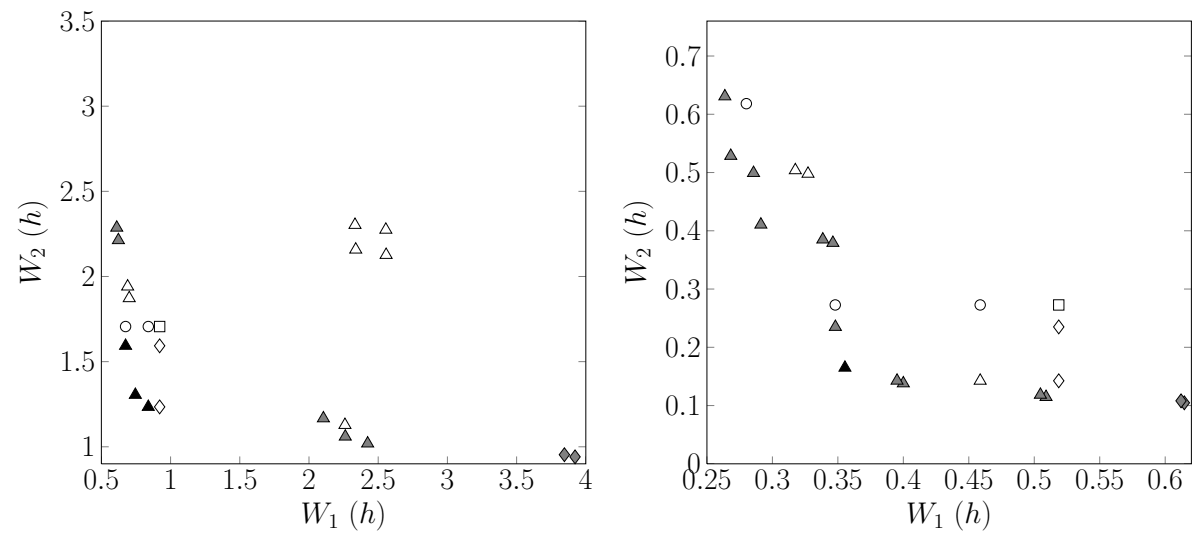

$$
\triangle \mathrm{EF}-\mathrm{NF} \quad \circ \mathrm{NF}-\mathrm{EF} \quad \square \mathrm{EF}-\mathrm{EF} \quad \triangle \mathrm{NF}-\mathrm{NF}
$$

$(1,4)$. There are two possible working routines for each doctor, thus the total number of RPCs investigated is $19 \times 4=76$.

Basic performance measures We refer to the $\operatorname{RPC}(N F, 5, N F, 5)$ as the current situation; both doctors visit new patients first and are allowed to use all treatment rooms in parallel. From the analytical model we obtain that for the current situation the expected LoS equals 3.4 hour for surgical patients, and $3.9 \mathrm{~h}$ for internal medicine patients. These values are significantly higher than the measured average LoS, as was expected since the peak arrival rate is used in the analytical model. In order to determine the Pareto efficient RPCs, we consider six objectives: three performance measures for both patient types. For each performance measure we determine the Pareto front from all RPCs; if an RPC is Pareto efficient it is impossible to establish an improvement for one patient type without increasing the blocking probability, the expected waiting time and/or the expected LoS for the other type. All RPCs that are not Pareto efficient are said to be dominated. There exist RPCs for which all three performance measures are Pareto efficient, which we denote by 'efficient' RPCs.

The Pareto front for the expected waiting time is depicted in Figure 10.3. Here all markers indicate one or multiple RPCs and the different marker types indicate the different working routines. One marker can indicate multiple RPCs; for example for the routine where both doctors treat existing patients first (EF- 
EF, marker square), both doctors will by assumption never occupy more than two rooms in parallel, resulting in the same expected waiting time for all policies with $\delta_{j} \geq 2$.

Both for the blocking probability and the expected LoS the Pareto front consists of a single $\left(P_{1}^{b}, P_{2}^{b}\right)$ - and $\left(S T_{1}, S T_{2}\right)$-coordinate respectively, and this optimum is attained in the policies $\left(\delta_{1}, \delta_{2}\right) \in\{(3,3) ;(3,2) ;(2,3)\}$ for all working routines.

For the JBH case study, the efficient RPCs and the resulting performance measures are given in Table 10.2. It appears that the improvements when

Table 10.2 Waiting times for the current situation (1st line) and the efficient RPCs, all with routine NF-NF.

\begin{tabular}{ccllllll}
\hline$\delta_{1}$ & $\delta_{2}$ & $W_{1}(h)$ & $W_{2}(h)$ & $S T_{1}(h)$ & $S T_{2}(h)$ & $P_{1}^{b}$ & $P_{2}^{b}$ \\
\hline 5 & 5 & 2.56 & 2.27 & 3.39 & 3.89 & 0.18 & 0.19 \\
3 & 3 & $0.75(-71 \%)$ & $1.30(-43 \%)$ & $1.34(-60 \%)$ & $2.53(-35 \%)$ & $0.01(-94 \%)$ & $0.07(-64 \%)$ \\
3 & 2 & $0.67(-74 \%)$ & $1.59(-30 \%)$ & $1.34(-60 \%)$ & $2.53(-35 \%)$ & $0.01(-94 \%)$ & $0.07(-64 \%)$ \\
2 & 3 & $0.84(-67 \%)$ & $1.23(-46 \%)$ & $1.34(-60 \%)$ & $2.53(-35 \%)$ & $0.01(-94 \%)$ & $0.07(-64 \%)$ \\
\hline
\end{tabular}

efficient policies are used are significant, especially for type 1 patients. The efficient policies all require both doctors to visit new patients first when a patient finishes (a phase of) his treatment. This policy implies that both doctors occupy as many treatment rooms in parallel as possible, which is beneficial for the waiting time of patients. The optimal room assignment policies indicate in all cases that one doctor should never use less than two rooms in parallel, which ensures that the doctor does not have to be inactive while waiting for test results. The room assignment policies $(3,2)$ and $(2,3)$ ensure that each doctor has his own treatment rooms, and no rooms are used by both doctors. For this case study sharing all the available rooms, which is the current room assignment policy at the JBH, appears to result in higher blocking probability, waiting time and/or LoS for patients.

Simulation results From the simulation study, as expected, it appears that the value of all performance indicators decreases when time-varying arrival rates are incorporated. For example, for the current policy the average LoS in the simulation is $1.1 \mathrm{~h}$ for type 1 patients and $1.6 \mathrm{~h}$ for type 2 patients, and the average LoSs with the Pareto efficient RPCs are $1.0 \mathrm{~h}$ and $1.2 \mathrm{~h}$ respectively. The improvement achieved with the Pareto efficient RPCs is less than in the analytical results: $-6 \%$ and $-26 \%$ respectively.

Similar to the results of the analytical model, in the simulation results a single $\left(P_{1}^{b}, P_{2}^{b}\right)$-coordinate forms the Pareto front for the blocking probability. For the average LoS, three Pareto efficient coordinates exist; of these coordinates two are uniquely attained and one is attained in $30 \mathrm{RPCs}$.

For $94 \%$ of the $(3$ indicators $\times 76$ RPCs $=) 228$ performance indicators, the conclusion whether the indicator is Pareto efficient is the same in the simulation results and analytical results. From the analytical model three RPCs appear to be efficient (see Table 10.2), but only $(\mathrm{NF}, 3, \mathrm{NF}, 3)$ is efficient in the simulation 
results.

Conditional performance measures We study the conditional expected LoS when the doctors both visit a new patient first at phase completion, since the JBH already works according to this routine, and from the analytical model it appears to be the only routine with Pareto efficient RPCs. Figures 10.4 and 10.5 display the conditional expected LoS of both patient types for different system states with $n_{2}=5, r_{2}=1, i_{2}=1$ (a typical example) when, respectively, room assignment policies $(3,3)$ and $(4,2)$ would be implemented and both doctors see new patients first.

The conditional expected LoS for type 1 patients increases with the number of patients present upon arrival, and decreases with the number of rooms that the type 1 doctor occupies for states with relatively few type 1 patients. The conditional expected LoS of a type 2 patient is almost equal for all system states in Figure 10.4. However, for policies that are not efficient, there is a difference for states with relatively many rooms; compare for example states with $n_{1}=6$ and $r_{1}=3,4$ in Figure 10.5. From this figure it also appears that the expected LoS of type 2 patients is higher when there are few type 1 patients at arrival, because for these states the type 2 doctor can take an additional room and as a consequence the LoS of all existing patients increases. Moreover, the conditional expected LoS of a type 2 patient is higher when the type 1 doctor occupies more rooms at the instant the patient arrives.

The national norm on the LoS, including time for triage, is 3 hours. From Figure 10.4 we may conclude that the expected LoS of type 1 patients will exceed the norm if they arrive when there are already 10 patients of their type present and the ED doctors would both visit new patients first and use at most 3 rooms in parallel. In order to provide insight about the effect of using the optimal room assignment policy, we simulated the system for all room assignment policies and the same working routine (NF-NF). The results for room assignment policy $(4,2)$ are displayed in Figure 10.5, which is a typical result for a non-optimal policy. For the $(4,2)$ policy, the norm will be exceeded for type 1 patients arriving in a system with 12 patients of their type present. For both policies, type 2 patients will never have an expected LoS exceeding the norm, but as the conditional expected LoS depends more on the number of type 2 patients in the system, it may exceed the norm for other values of $\left(n_{2}, r_{2}, i_{2}\right)$.

\subsubsection{Additional insights}

We investigated several additional parameter settings in systems with a different number of rooms, in order to determine whether the insights found for the JBH case study also hold in general. In this subsection we present the most insightful results, based on a system with eight treatment rooms for four cases (see Table 10.3). In cases 1 and 2 the expected consultation time of type 1 patients is equal, but in case 2 the expected number of visits by the doctor is lower. In 
10.5. Numerical results

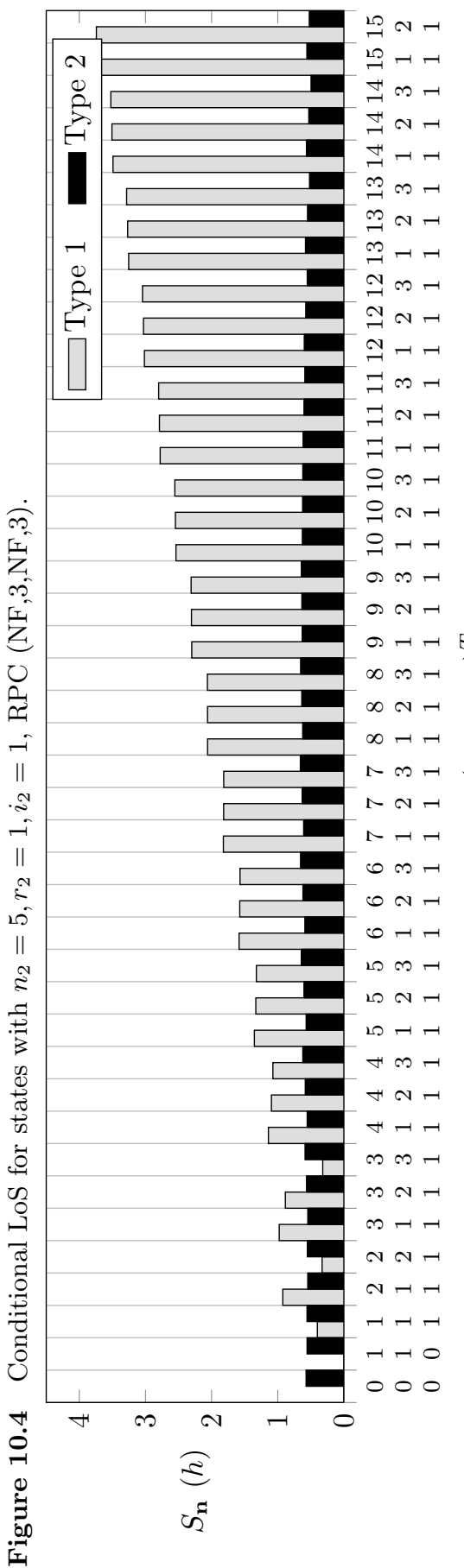

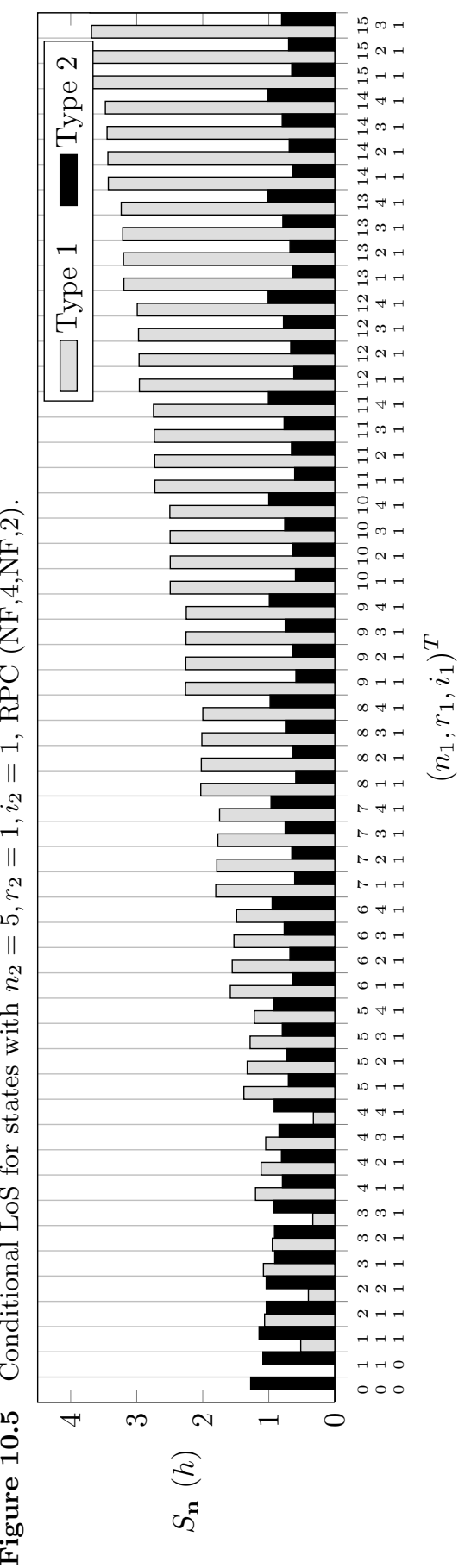


Table 10.3 Input parameters for cases with eight treatment rooms.

\begin{tabular}{c|ccccc|ccccc}
\hline Case & $\lambda_{1}$ & $\mu_{1}$ & $\nu_{1}$ & $p_{1}$ & $M_{1}$ & $\lambda_{2}$ & $\mu_{2}$ & $\nu_{2}$ & $p_{2}$ & $M_{2}$ \\
\hline 1 & 6.00 & 12.00 & 2.00 & 0.67 & 15 & 2.00 & 7.50 & 2.00 & 0.33 & 10 \\
2 & 6.25 & 9.26 & 2.00 & 0.90 & 15 & 2.00 & 7.50 & 2.00 & 0.33 & 10 \\
3 & 2.00 & 7.50 & 2.00 & 0.33 & 10 & 2.00 & 7.50 & 2.00 & 0.33 & 10 \\
4 & 6.00 & 12.00 & 2.00 & 0.67 & 15 & 2.00 & 7.50 & 1.00 & 0.33 & 10 \\
\hline
\end{tabular}

case 3 both type 1 and 2 patients have exactly the same characteristics. Case 4 is equal to case 1 except for $\nu_{2}$.

From the analytical results it appears that also for the system with a different number of treatment rooms, the Pareto-front for the blocking probability and LoS consists of one coordinate. The results for all cases of Table 10.3 are displayed in Table 10.4, in which 'Current' implies $\left(\delta_{1}, \delta_{2}\right)=(8,8)$ and the extreme values of the Pareto-front of the expected waiting time are given instead of all individual coordinates. The efficient working routine is NF-NF in all cases, and the efficient room assignment policies are displayed in Table 10.5.

Table 10.4 Results from the analytical model for eight treatment rooms.

\begin{tabular}{|c|c|c|c|c|c|c|c|}
\hline & & $W_{1}(h)$ & $W_{2}(h)$ & $S T_{1}(h)$ & $S T_{2}(h)$ & $P_{1}^{b}$ & $P_{2}^{b}$ \\
\hline \multirow{2}{*}{ Case 1} & Current & 0.33 & 0.35 & 0.69 & 1.85 & 0.025 & 0.033 \\
\hline & Efficient & $(0.19,0.30)$ & $(0.28,0.53)$ & 0.52 & 1.74 & 0.004 & 0.027 \\
\hline \multirow[t]{2}{*}{ Case 2} & Current & 0.37 & 0.24 & 0.59 & 1.76 & 0.018 & 0.028 \\
\hline & Efficient & $(0.24,0.29)$ & $(0.28,0.94)$ & 0.47 & 1.74 & 0.003 & 0.027 \\
\hline \multirow[t]{2}{*}{ Case 3} & Current & 0.49 & 0.49 & 1.94 & 1.94 & 0.039 & 0.039 \\
\hline & Efficient & $(0.33,0.55)$ & $(0.33,0.55)$ & 1.74 & 1.74 & 0.027 & 0.027 \\
\hline \multirow[t]{2}{*}{ Case 4} & Current & 0.33 & 0.36 & 0.69 & 1.90 & 0.025 & 0.035 \\
\hline & Efficient & $(0.17,0.30)$ & $(0.28,0.54)$ & 0.52 & 1.77 & 0.004 & 0.027 \\
\hline
\end{tabular}

Table 10.5 Efficient policies for eight treatment rooms with working routine NF-NF.

\begin{tabular}{cl}
\hline Case & Policies $\left(\delta_{1}, \delta_{2}\right)$ \\
\hline 1 & $(6,6)^{*}(6,5)(6,4)(5,6)^{*}(4,6)^{*}(3,6)(2,6)$ \\
2 & $(6,6)^{*}(6,5)^{*}(6,4)^{*}(6,3)(6,2)(5,6)^{*}(4,6)(3,6)^{*}(2,6)$ \\
3 & $(6,6)^{*}(6,5)^{*}(6,4)(5,6)(4,6)$ \\
4 & $(6,6)^{*}(6,5)(6,4)(5,6)(4,6)^{*}(3,6)^{*}(2,6)$ \\
${ }^{*}=$ & RPC is also efficient according to simulation results.
\end{tabular}

From Table 10.4 it appears that for both patient types it is better if one of them requires less phases that each take longer, compare cases 1, 2 and 3 . However, when the rooms are divided according to the efficient assignment policies there is only an improvement for type 1 patients. When type 2 patients have to wait longer for the test results, this only increases their expected waiting time and LoS and does not affect type 1 patients. From Table 10.5 it appears that all 
doctors should get at least two treatment rooms in parallel.

For all cases the performance of the system significantly improves when the efficient room assignment policies are used. In cases 1, 2 and 4 the expected LoS of type 1 patients decreases more than $20 \%$ and the blocking probability more than $80 \%$. For case 3 the decrease is $10 \%$ for the expected LoS and $31 \%$ for the blocking probability.

We validated the results again by means of the simulation model with timevarying arrival rates. Most of the Pareto efficient RPCs are also found in the simulation results; $94 \%$ of the conclusions whether a RPC is optimal for a performance measure is the same in the results of both models. As expected, all performance measures are lower in the simulation results. The improvements by introducing an efficient RPC are in cases 1,2 and 4 over $60 \%$ for the blocking probability and over $8 \%$ for the expected LoS of type 1 patients. For case 3 the decrease is over $10 \%$ for the blocking probability and $3 \%$ for the expected LoS. In addition to the efficient policies in Table 10.5, the simulation results indicate that policy $(\mathrm{NF}, 8, \mathrm{NF}, 3)$ and all policies with $\delta_{1}=7$ and $\delta_{2} \geq 2$ for routine NF-EF are efficient. We postulate that also for other parameter settings the performance measures can be improved significantly by implementing an RPC, and the efficient RPCs found by means of the analytical model are also found to be efficient in a system with time-varying arrival rates.

\subsection{Discussion}

The assignment of treatment rooms among doctors at EDs is often unbalanced, which possibly affects patients' blocking probability, waiting time and LoS. From the results of this study, we conclude that introducing threshold policies that indicate the maximum number of rooms that one doctor can use in parallel, can significantly improve the performance of the ED for all relevant performance measures. When two doctors interact on sharing treatment rooms, the working routine to visit new patients first is found to result in the best performance.

We analytically expressed the expected LoS of a patient conditioned on the state of the system this patient encounters upon arrival. This conditional waiting time and LoS information may be provided to the patients to inform them about their expected length of stay at the ED, which adds to patient satisfaction.

One limitation of the analytical model is that it suffers from an 'exploding state space'; it could be extended to incorporate more patient types, but for determining the conditional LoS this will make the state space too large and/or computation time very long. In the analytical model we assume stationary arrival rates, which is typically not the case at an ED. Our simulation study indicates that this assumption does not affect the decisions whether a policy is optimal or not. Other limiting assumptions made in this model are: (1) no emergency arrivals, (2) doctors visiting their patients in random order, (3) when a doctor occupies two or more treatment rooms in parallel, the test results will be ready before the next phase completion of one of his patients, (4) the probability that a 
patient can go home after a phase completion is equal for all phases, and (5) the duration of one phase of service is exponentially distributed. These assumptions are included for analytical tractability, but are not realistic for ED systems. To overcome this limitation and investigate to what extent these assumptions affect the policy that results in the best performance, in Chapter 11 we investigate this system using a rolling horizon approach.

\subsection{Appendix A The generator}

We will illustrate part of the generator matrix of the system described in Section 10.3 for the working routine in which both doctors visit a new patient first at phase completion. For all other working routines and generator entries, the generator matrix can be obtained in similar ways. Define additional parameters:

$c$ total number of rooms,

$M_{j}$ maximum number of patients in system, $j=1,2$,

$\delta_{j}$ the maximum number of rooms occupied, $j=1,2$,

$\nu$ the parameter of the exponential time a doctor has to wait on test results.

There are six possible events when the system is in state $\left(n_{1}, r_{1}, 1 ; n_{2}, r_{2}, 1\right)$ : an arrival, departure and phase completion for both patient types. In this chapter, $\mathbb{1}\{x\}$ equals 1 if event $x$ is true and 0 otherwise; $\mathbb{1}\{x, y\}$ equals 1 if both $x$ and $y$ are true; and $\mathbb{1}\{x \| y\}$ equals 1 if $x$ or $y$ is true. Let $\mathbf{n}=\left(n_{1}, r_{1}, 1 ; n_{2}, r_{2}, 1\right)$ be a state with $1<n_{1}<M_{1}$ and $1<n_{2}<M_{2}$, then:

$\mathbf{Q}\left(\mathbf{n} ; \mathbf{n}^{\prime}\right)=$

$$
\left\{\begin{array}{l}
\lambda_{1} \\
\lambda_{2} \\
p_{1} \mu_{1} \mathbb{1}\left\{n_{1}=r_{1}\right\} \\
p_{1} \mu_{1} \mathbb{1}\left\{n_{1}>r_{1}\right\} \\
\\
p_{2} \mu_{2} \mathbb{1}\left\{n_{2}=r_{2}\right\} \\
p_{2} \mu_{2} \mathbb{1}\left\{n_{2}>r_{2}\right\} \\
\left(1-p_{1}\right) \mu_{1} \mathbb{1}\left\{n_{1}>r_{1}, r_{1}<\delta_{1}, r_{1}+r_{2}<c\right\} \\
\left(1-p_{2}\right) \mu_{2} \mathbb{1}\left\{n_{2}>r_{2}, r_{2}<\delta_{2}, r_{1}+r_{2}<c\right\} \\
\left(1-p_{1}\right) \mu_{1} \mathbb{1}\left\{n_{1}=r_{1}\left\|r_{1}=\delta_{1}\right\| r_{1}+r_{2}=c\right\} \\
\quad+\left(1-p_{2}\right) \mu_{2} \mathbb{1}\left\{r_{2}=\delta_{2}\left\|n_{2}=r_{2}\right\| r_{1}+r_{2}=c\right\}
\end{array}\right.
$$

$$
\begin{aligned}
& \mathbf{n}^{\prime}=\left(n_{1}+1, r_{1}, 1 ; n_{2}, r_{2}, 1\right) \\
& \mathbf{n}^{\prime}=\left(n_{1}, r_{1}, 1 ; n_{2}+1, r_{2}, 1\right) \\
& \mathbf{n}^{\prime}=\left(n_{1}-1, r_{1}-1,1 ; n_{2}, r_{2}, 1\right) \\
& \mathbf{n}^{\prime}=\left(n_{1}-1, r_{1}, 1 ; n_{2}, r_{2}, 1\right) \\
& \mathbf{n}^{\prime}=\left(n_{1}, r_{1}, 1 ; n_{2}-1, r_{2}-1,1\right) \\
& \mathbf{n}^{\prime}=\left(n_{1}, r_{1}, 1 ; n_{2}-1, r_{2}, 1\right) \\
& \mathbf{n}^{\prime}=\left(n_{1}, r_{1}+1,1 ; n_{2}, r_{2}, 1\right) \\
& \mathbf{n}^{\prime}=\left(n_{1}, r_{1}, 1 ; n_{2}, r_{2}+1,1\right) \\
& \mathbf{n}^{\prime}=\left(n_{1}, r_{1}, 1 ; n_{2}, r_{2}, 1\right)
\end{aligned}
$$


For a state with $n_{1}=r_{1}=n_{2}=r_{2}=1$ we have:

$$
\mathbf{Q}\left(\mathbf{n} ; \mathbf{n}^{\prime}\right)= \begin{cases}\lambda_{1} \mathbb{1}\left\{i_{1}=0, r_{1}<\delta_{1}, r_{1}+r_{2}<c\right\} & \mathbf{n}^{\prime}=\left(n_{1}+1, r_{1}+1,1 ; n_{2}, r_{2}, i_{2}\right) \\ \lambda_{1} \mathbb{1}\left\{i_{1}=1\left\|r_{1}=\delta_{1}\right\| r_{1}+r_{2}=c\right\} & \mathbf{n}^{\prime}=\left(n_{1}+1, r_{1}, i_{1} ; n_{2}, r_{2}, i_{2}\right) \\ \lambda_{2} \mathbb{1}\left\{i_{2}=0, r_{2}<\delta_{2}, r_{1}+r_{2}<c\right\} & \mathbf{n}^{\prime}=\left(n_{1}, r_{1}, i_{1} ; n_{2}+1, r_{2}+1,1\right) \\ \lambda_{2} \mathbb{1}\left\{i_{2}=1\left\|r_{2}=\delta_{2}\right\| r_{1}+r_{2}=c\right\} & \mathbf{n}^{\prime}=\left(n_{1}, r_{1}, i_{1} ; n_{2}+1, r_{2}, i_{2}\right) \\ p_{1} \mu_{1} \mathbb{1}\left\{i_{1}=1,\left\{i_{2}=1 \| r_{2}=\delta_{2}\right\}\right\} & \mathbf{n}^{\prime}=\left(n_{1}-1, r_{1}-1,0 ; n_{2}, r_{2}, i_{2}\right) \\ p_{1} \mu_{1} \mathbb{1}\left\{i_{1}=1, i_{2}=0, r_{2}<\delta_{2}\right\} & \mathbf{n}^{\prime}=\left(n_{1}-1, r_{1}-1,0 ; n_{2}, r_{2}+1,1\right) \\ p_{2} \mu_{2} \mathbb{1}\left\{i_{2}=1,\left\{i_{1}=1 \| r_{1}=\delta_{1}\right\}\right\} & \mathbf{n}^{\prime}=\left(n_{1}, r_{1}, i_{1} ; n_{2}-1, r_{2}-1,0\right) \\ p_{2} \mu_{2} \mathbb{1}\left\{i_{2}=1, i_{1}=0, r_{1}<\delta_{1}\right\} & \mathbf{n}^{\prime}=\left(n_{1}, r_{1}+1,1 ; n_{2}-1, r_{2}-1,0\right) \\ \left(1-p_{1}\right) \mu_{1} \mathbb{1}\left\{i_{1}=1\right\} & \\ \left(1-p_{2}\right) \mu_{2} \mathbb{1}\left\{i_{2}=1\right\} & \mathbf{n}^{\prime}=\left(n_{1}, r_{1}, 0 ; n_{2}, r_{2}, i_{2}\right) \\ \nu_{1} \mathbb{1}\left\{i_{1}=0\right\} & \mathbf{n}^{\prime}=\left(n_{1}, r_{1}, i_{1} ; n_{2}, r_{2}, 0\right) \\ \nu_{2} \mathbb{1}\left\{i_{2}=0\right\} & \\ & \mathbf{n}^{\prime}=\left(n_{1}, r_{1}, 1 ; n_{2}, r_{2}, i_{2}\right)\end{cases}
$$

Here, the first four lines represent arrivals; lines 5-8 represent departures; lines 9 and 10 represent phase completions without departures; and the last two lines represent the doctors returning to their patients after waiting for the test results.

The generator entries for other states are obtained following the same reasoning. The diagonal entries of $\mathbf{Q}$ are such that all row sums equal zero.

\subsection{Appendix B Conditional performance measures}

We next summarize the steps in obtaining the conditional expected LoS of a patient. More details on this method can for example be found in [313, 410]. Recall that the expected LoS of an admitted patient, conditioned on the state of the system at the instant the patient has arrived, is expressed by

$$
S T_{\mathbf{n} j}=\int_{0}^{\infty} \sigma_{\mathbf{n} j}(x) f_{j}(x) \mathrm{d} x, \text { with } \sigma_{\mathbf{n} j}(x)=\alpha_{\mathbf{n} j}+\sum_{\mathbf{n}^{\prime}} \tau_{\mathbf{n}^{\prime} j}(x) \psi_{j}\left(\mathbf{n}, \mathbf{n}^{\prime}\right) .
$$

The following subsections respectively describe the analysis of the conditional expected waiting time $\alpha_{\mathbf{n}}$, the conditional expected treatment time $\tau_{\mathbf{n}}(x)$, and the transition probabilities $\psi\left(\mathbf{n}, \mathbf{n}^{\prime}\right)$.

\subsubsection{The conditional expected waiting time}

The conditional expected waiting time of a tagged type $j$ patient that has just arrived, and upon arrival encountered a certain state $\mathbf{n}$, can be determined by considering an alternative system equal to the original system, but with the arrival rate of type $j$ patients equal to zero. The time it takes for the alternative system to reach a state in which the queue for type $j$ patients is empty equals the waiting time of a type $j$ patient in the original system, since this implies the tagged patient will start his service. 
For completeness, we summarize the results mentioned in [313]. Let $\tilde{\mathbf{Q}}$ denote the generator of the process obtained by letting $\lambda_{j}=0$, and removing all states with an empty type $j$ queue. The states with no type $j$ patients queued represent the absorbing set. The time until absorption can then readily be determined by $\operatorname{defining} \tilde{\alpha}_{\mathbf{n}}:=-1 / \operatorname{diag}(\tilde{\mathbf{Q}})$, with $\operatorname{diag}(\tilde{\mathbf{Q}})$ the $\operatorname{diagonal}$ of $\tilde{\mathbf{Q}}$. Let $\tilde{\mathbf{P}}$ be the onestep transition probability matrix of the embedded jump chain of the process described by generator $\tilde{\mathbf{Q}}$. Then,

$$
\alpha_{\mathbf{n}}=(\boldsymbol{I}-\tilde{\mathbf{P}})^{-1} \tilde{\alpha}_{\mathbf{n}}
$$

with $\boldsymbol{I}$ an identity-matrix of appropriate size. The inverse of $(\boldsymbol{I}-\tilde{\mathbf{P}})$ exists since $\tilde{\mathbf{P}}$ is by definition a substochastic matrix.

\subsubsection{The conditional expected treatment time}

The expected treatment time of a tagged patient with consultation time $x$ entering a treatment room in state $\mathbf{n}, \tau_{\mathbf{n}}(x)$, can be described by a system of differential equations, in which we assume the tagged patient cannot leave the system for some small time $\Delta$. We augment the state space by $z \in\{0,1\}$, where $z=1$ indicates the doctor is with the tagged patient.

We now formulate the differential equations for the conditional expected treatment time by conditioning on a small time $\Delta$ during which the tagged patient cannot complete its treatment. Note that the doctor still occasionally visits the tagged patient as if it were a regular patient, but after a visit the tagged patient will not leave the system. For the conditional expected treatment of a type 1 patient that is currently seen by the doctor in state $\tilde{\mathbf{n}}=\left(n_{1}, r_{1}, i_{1} ; n_{2}, r_{2}, 1 ; 1\right)$ with $n_{1}, n_{2}>1, r_{1}+r_{2}<c$ ( $c$ the total number of treatment rooms), we get

$$
\begin{aligned}
\tau_{\tilde{\mathbf{n}}}(x)= & \Delta \\
& +\lambda_{1} \Delta \tau_{\tilde{\mathbf{n}}+\mathbf{e}_{1}}(x-O(\Delta)) \\
& +\left(1-p_{1}\right) \mu_{2} \Delta \tau_{\tilde{\mathbf{n}}+\mathbf{e}_{2}-\mathbf{e}_{7}}(x-O(\Delta)) \\
& +\lambda_{2} \Delta \tau_{\tilde{\mathbf{n}}+\mathbf{e}_{4}}(x-O(\Delta)) \\
& +p_{2} \mu_{2} \Delta \tau_{\tilde{\mathbf{n}}-\mathbf{e}_{4}}(x-O(\Delta)) \\
& +\left(1-p_{2}\right) \mu_{2} \Delta \tau_{\tilde{\mathbf{n}}+\mathbf{e}_{5}}(x-O(\Delta)) \\
& +\left(1-\lambda_{1}-\left(1-p_{1}\right) \mu_{1}-\lambda_{2}-\mu_{2}\right) \Delta \tau_{\tilde{\mathbf{n}}}(x-\Delta) \\
& +o(\Delta)
\end{aligned}
$$

Here $\mathbf{e}_{k}$ denotes a vector with value 1 at position $k$ and zeros elsewhere. The second and fourth line on the right-hand side represent that in time $\Delta$ an arrival of type 1 and 2 has occurred, to a state with one additional type 1 patient $\left(\tilde{\mathbf{n}}+\mathbf{e}_{1}\right)$ and one additional type 2 patient $\left(\tilde{\mathbf{n}}+\mathbf{e}_{4}\right)$, respectively. Line three refers to a completion of the current phase of the tagged patient, where the doctor gets an extra room and the service of the tagged patient is temporarily 
paused $\left(\tilde{\mathbf{n}}+\mathbf{e}_{2}-\mathbf{e}_{7}\right)$. Line five represents a departure of type $2\left(\tilde{\mathbf{n}}-\mathbf{e}_{4}\right)$, and line six a phase completion of a type 2 patient where the type 2 doctor treats a new patient in an additional treatment room $\left(\tilde{\mathbf{n}}+\mathbf{e}_{5}\right)$. The last two lines represent, respectively, that nothing happens and that two events happen in time $\Delta$. The equations for states with $z=0$ and boundary states can be obtained in similar ways. We then obtain the derivative of $\tau_{\tilde{\mathbf{n}}}(x)$ by rearranging terms and letting $\Delta \rightarrow 0$.

This system of differential equations may equivalently be written in matrix notation. To this end, we introduce generator $\mathbf{Q}^{*}$, which is identical to $\mathbf{Q}$ excluding all states with $n_{j}=0$ and the departure rate modified such that the permanent patient never leaves the system. All diagonal elements of $\mathbf{Q}^{*}$ are such that each row sums to zero. Since there are states in which the service rate of the tagged patient equals 0 , we reorder the states such that the state space can be split into states with $z=1$ (subscript + ) and with $z=0$ (subscript 0 ). We write $\mathbf{Q}^{*}$ as follows:

$$
\mathbf{Q}^{*}=\left[\begin{array}{ll}
\mathbf{Q}_{+}^{*} & \mathbf{Q}_{+\mathbf{0}}^{*} \\
\mathbf{Q}_{\mathbf{0}+}^{*} & \mathbf{Q}_{\mathbf{0}}^{*}
\end{array}\right]
$$

The system of differential equations can then be written as

$$
\frac{\partial}{\partial x} \tau_{+}(x)=\mathbf{1}+\mathbf{Q}_{+\mathbf{0}}^{*} \tau_{\mathbf{0}}(x)+\mathbf{Q}_{+}^{*} \tau_{+}(x),
$$

with 1 a vector with all entries equal to one. The initial condition of this system is $\tau_{+}(0)=0$, which indicates that the conditional expected treatment time of a patient with consultation time zero equals zero almost surely. Here $\tau_{\mathbf{0}}, \tau_{+}$ are vectors containing $\tau_{\tilde{\mathbf{n}}}$ for states with $z=0$ and $z=1$ respectively, and $\tau(x)=\left(\tau_{+}(x), \tau_{\mathbf{0}}(x)\right)$. This system is similar to a system with one permanent patient.

Let $\pi_{+}^{*}$ be a stationary distribution obtained by solving $\pi_{+}^{*} \mathbf{Q}_{+}^{*}=\mathbf{0}$. Then, the conditional expected treatment time of a patient with consultation time $x$ is given by:

$$
\begin{aligned}
\tau_{+}(x) & =x \mathbf{1}+\left[\boldsymbol{I}-\exp \left(x\left\{\mathbf{Q}_{+}^{*}-\mathbf{Q}_{+\mathbf{0}}^{*}\left(\mathbf{Q}_{\mathbf{0}}^{*}\right)^{-1} \mathbf{Q}_{\mathbf{0}+}^{*}\right\}\right)\right] \gamma, \\
\tau_{\mathbf{0}}(x) & =-\left(\mathbf{Q}_{\mathbf{0}}^{*}\right)^{-1}\left(\mathbf{1}+\mathbf{Q}_{\mathbf{0}+}^{*} \tau_{+}(x)\right)
\end{aligned}
$$

with $\gamma$ the unique solution of

$$
\begin{aligned}
-\left\{\mathbf{Q}_{+}^{*}-\mathbf{Q}_{+\mathbf{0}}^{*}\left(\mathbf{Q}_{\mathbf{0}}^{*}\right)^{-1} \mathbf{Q}_{\mathbf{0}+}^{*}\right\} \gamma & =\mathbf{Q}_{+\mathbf{0}}^{*}\left(\mathbf{Q}_{\mathbf{0}}^{*}\right)^{-1} \mathbf{1}, \\
\pi_{+}^{*} \gamma & =0 .
\end{aligned}
$$

Following the derivation in [313], we may show that (10.4) has a unique solution, and it is readily checked that (10.5) is the solution. 


\subsubsection{Transition probabilities}

The conditional expected access and treatment time have to be linked together by the probability that a patient arriving in state $\mathbf{n}$ starts his service in state $\mathbf{n}^{\prime}$. By analogy with the derivation above, we obtain the probability matrix $\psi$ by tagging a patient and augmenting the state space with the location $l$ of the tagged patient, which equals zero if the patient is no longer queued. In this system, all states where the tagged patient is no longer queued form an absorbing set. For completeness, we summarize these results from [313]. Denote with $\mathbf{P}^{\bullet}$ the onestep probability matrix of the embedded jump chain of the augmented Markov chain. Then, for all states $\mathbf{n}$ in the absorbing set $\mathbf{P}^{\bullet}(0, \mathbf{n} ; 0, \mathbf{n})=1$. For all other states, $\mathbf{P}^{\bullet}\left(l, \mathbf{n}, l^{\prime}, \mathbf{n}^{\prime}\right)$ is non-zero only if $\mathbf{Q}\left(\mathbf{n}, \mathbf{n}^{\prime}\right)>0$ and there is a transition from $l$ to $l^{\prime}$. $\mathbf{P}^{\bullet}$ can be written as

$$
\mathbf{P}^{\bullet} \equiv\left(\begin{array}{cc}
\boldsymbol{I} & \mathbf{O} \\
\mathbf{P}_{\mathbf{0}}^{\bullet} & \mathbf{P}_{+}^{\bullet}
\end{array}\right)
$$

with $\mathbf{O}$ the null-matrix, $\mathbf{P}_{\mathbf{0}}^{\bullet}$ a stochastic submatrix corresponding to transitions from the recurrent states into the absorbing set, and $\mathbf{P}_{+}^{\bullet}$ transitions among the recurrent states. We obtain the probability matrix $\psi$ using $\psi\left(\mathbf{n}, \mathbf{n}^{\prime}\right)=$ $\psi^{\bullet}\left(l, \mathbf{n} ; 0, \mathbf{n}^{\prime}\right)$, and $\psi^{\bullet}=\left(\boldsymbol{I}-\mathbf{P}_{+}^{\bullet}\right)^{-1} \mathbf{P}_{\mathbf{0}}^{\bullet}$, where the inverse always exists since $\mathbf{P}_{\mathbf{0}}^{\bullet}$ is substochastic, see [313]. 


\section{1}

\section{Sequentially assigning and prioritizing patients at emergency departments}

\subsection{Introduction}

Medical doctors at emergency departments (EDs) relatively often treat severely ill patients, and logistical inefficiencies at EDs may result in life-threatening situations quicker than at other hospital departments. These inefficiencies may for example occur due to long waiting times for diagnostic tests, or due to a lack of inpatient beds or treatment rooms. Additionally, at EDs one doctor typically occupies multiple rooms in parallel; when patients are awaiting diagnostic test results, the doctor treats other patients. From Chapter 10 it appears that treating too few or too many patients in parallel also negatively affects the ED's performance.

Both prioritizing patients and assigning patients to doctors significantly affect patients' length of stay (LoS) at the ED. Each time a doctor completes (a phase of) a patient's treatment time, she has the choice between treating a new patient from the waiting area or an existing patient who is already treated before. Due to their different specializations, not all doctors can treat all patients and treatment times for each patient type may differ among doctors. Additionally, patients are preferably treated by a single doctor for all the phases of their treatment. These factors make assigning and prioritizing patients efficiently a complicated task, which is in practice often performed by the triage nurse or doctors.

In Chapter 10 we investigated several room assignment policies and doctor working routines. Room assignment policies limit the number of rooms each doctor occupies in parallel. The working routines prescribe if a doctor should give priority to an existing or a new patient. Among the investigated combinations of room assignment policies and working routines, prioritizing new patients appeared to result in the best performance. The best room assignment policy depends on the system parameters.

In this chapter we aim to optimize the priority policy of the doctor and the patient-doctor assignments in each state of the ED system. By using a rolling-horizon approach, we are able to incorporate time-varying arrival rates. Furthermore, we take each doctor's specialization into account, and each phase of treatment may have different characteristics. The objective of the optimization 
is to minimize patients' LoS at the ED. Additionally, we investigate the time until a patient is first treated by a doctor (waiting time) and the number of patients that leave the ED within the national norm on the LoS. This chapter is organized as follows. First, we provide an overview of related literature in Section 11.2. Second, we analyze extreme cases of the patient prioritizing problem in Section 11.3. We present the model in Section 11.4, and end with a presentation of our results and a conclusion.

\subsection{Related literature}

In this section we provide a brief overview of three related fields of literature. The first field of literature focuses on prioritizing patients at EDs, the second on dual resource constrained systems, and the third on the optimal assignment of flexible servers.

Most of the related literature on prioritizing patients at EDs considers different ways to triage patients, and let the triage category determine in which order the patients should be treated. In order to comply with LoS targets, often patients' LoSs are part of the input for the triage model and the triage categories are frequently updated. Recently examples of such triage systems are the 'group technology algorithm' [20] and a multi-attribute utility theory approach [109].

Three recent manuscripts consider a topic relatively close to the topic of the current chapter, as they consider doctors that may visit their patients multiple times. Kim and Kim [265] consider a queueing network in which each queue represents a stage in a patient's stay at the ED, such as 'triage', 'Xray' and 'CT scan'. Patients have probabilistic routes to visit queues in the network (and are not necessarily always treated by the same doctor), and the routing probabilities depend on the patient types. The authors assume there are multiple priority classes, and investigate different priority disciplines to reduce the waiting time of patients in higher priority classes. Huang et al. [237] consider an ED at which doctors have to choose between new and existing patients. The authors invoke a queueing model to obtain a policy that minimizes the weighted sum of the total LoS and the waiting times until the first consult with a doctor. He et al. [227] improve upon the policy in [237] using a robust optimization approach for the same objective.

A different related field of literature investigates dual resource constrained systems. The ED system under consideration in this chapter is such a system because a patient requires both a treatment room and doctor to be 'served'. From a recent literature review, it appears that these models are usually analyzed by means of simulation, genetic algorithms and neural networks [524]. In this collection of papers, the challenges are given in the five areas: job release mechanisms (effect of planning), job dispatching (determining the order of jobs to be processed), worker flexibility (preferring multi-skilled but slower workers or single-skilled quicker workers), worker assignment (which worker to which machine at what time) and transfer costs (letting machines switch from processing 
one type of jobs to another). In this chapter, the challenges are a mixture between release mechanisms, dispatching and worker assignment. One recent paper with a slightly different approach is on a dual resource constrained system with sequence and job dependent set-up times [123]. The authors use a mixed integer programming approach to solve smaller instances of the manufacturing problem, and base genetic algorithms on its solutions to solve realistic instances. In this chapter we aim for different objectives and use a different approach compared to the typical objectives and approaches considered in the literature.

Physicians at EDs often have the choice between treating an existing patient and treating a new patient. Already over 30 years ago, König and Schmidt [278] studied a similar decision problem for a tandem queueing network in which a flexible server should be assigned to one of the queues. Since then, several extensions of this model were studied, such as switch-over times every time a server is re-assigned [152], and a system in which all queues have one dedicated server and you optimize the assignment of one general server to one of the queues [442]. For an ED system, Zayas-Caban et al. [538] invoke Markov decision theory to optimize the decision of a doctor between triaging or treating patients. The difference with the existing literature in this field is that our system is dual resource constrained, so the total number of customers in service aggregated for all queues is limited. Moreover, we assume that patients are temporarily unavailable between completing the first and starting the second phase of their treatment. Furthermore, in production systems and general queueing networks it is often assumed that multiple servers may work together on one job, and the service is faster when they do, which is generally not the case at EDs.

Opposed to the literature on prioritizing patients at EDs, in this chapter we incorporate the availability of treatment rooms in our model, which imposes a maximum on the number of patients seen in parallel. Similar to [227], we use a rolling horizon approach to be able to incorporate time-varying arrival rates, which are an important feature of ED arrivals but are often not incorporated in the analyses. Furthermore, we incorporate that each doctor may visit each patient multiple times, where (1) the duration of the phase of treatment, (2) the time it takes for the test results to be available after the current phase, and (3) the probability that the patient requires another phase after the current one, may depend on the current phase, the doctor and the patient's type.

\subsection{Extreme case analyses}

In this section we investigate three extreme cases of the patient prioritizing problem to obtain more insights in the optimal priority policy. First, we provide some results from general scheduling literature for EDs operated by a single doctor. For the second case we consider optimizing the LoS tail, which is an important performance measure of EDs; patients should often be treated within three or four hours, a legal target that differs per country. In the third case we optimize the ED's throughput for a single server system in heavy traffic. 
For systems with one doctor, the scheduling literature provides some insights to the patient prioritizing problem. If the doctor aims to minimize the expected LoS of patients, the shortest remaining treatment time discipline is shown to be optimal [437] for all treatment time distributions and arrival processes. This policy would imply that doctors have perfect a priori knowledge on treatment times and times to complete diagnostic tests for all patients, which is not a very realistic assumption. Furthermore, the 'earliest due date first' algorithm is optimal for minimizing the maximum lateness, which is the maximum difference between the LoS target and a patient's ED discharge [195]. If all patients have the same LoS target, this algorithm implies the doctor should always treat the patient with the highest LoS first.

In the second extreme case we assume that the time to complete a (series of) diagnostic test(s) is negligible. This implies that after completing one phase of treatment, all patients are immediately available for another treatment. This extreme case resembles the regular dual resource constrained systems, except dual resource constrained systems are often analyzed in a processor sharing service discipline. If we furthermore assume that the duration of each phase of treatment has a light-tailed distribution, Nair et al. [367] show that always treating patients with the highest LoS first is optimal for minimizing the tail of the LoS distribution. This implies that each doctor should only use one treatment room at a time, and perform all phases of treatment for one patient continuously. The sojourn time tail objective is also studied in a system with these assumptions in [227, 265], and [227] (Figure 5, page 27) includes numerical results that show that treating patients with the highest LoS first, i.e., the first come first served (FCFS) discipline, results in the best LoS-target compliance. For the single server ED system in which the doctor chooses between triaging or treating patients, Zayas-Caban et al. [538] also prove that FCFS is optimal.

As noted above, for the single doctor model, when the time to complete diagnostic tests is negligible, FCFS is optimal under a wide range of circumstances. However, if the time to complete diagnostic tests is not negligible then this may not be the case. To illustrate this, in the following we consider a much simplified model in the heavy traffic case.

The model assumes a single doctor with a fixed number of rooms $N \geq 2$. The doctor treats a single patient type, and each patient has maximally two phases of service, with an intermediate diagnostic phase. We assume that a patient in the first phase of their treatment is treated at rate $\mu_{1}$. At the end of that treatment, they depart with probability $p$, but with probability $1-p$ they need further diagnostic or assessment tests. The mean time to complete these is $1 / \mu_{d}$. We assume that the diagnostic test phase operates like a multi-server queue, which may be the case for, e.g., blood tests, where several people are available to perform the analyses. While the diagnostic tests are being performed patients still occupy their room. Once the test results are returned, the doctor may choose to treat the patient again; this second phase of treatment lasts a mean time of 
$1 / \mu_{2}$, after which the patient leaves the ED. The heavy traffic assumption we make is that as soon as a patient leaves a room, that room is occupied by a new patient. The doctor treats one patient at a time, and after the completion of each treatment phase, chooses the next patient to treat - if there are both new and existing patients in the system ready to be seen, then the doctor could choose either. We make the standard simplifying assumptions of exponential treatment times in each phase, and that treatment times and the probability of departing without requiring diagnostic tests are all independent of one another. The question is whether it may sometimes be optimal to treat a new patient, rather than an existing patient, for the objective of maximizing the throughput. (As we assume the queue is always full, LoS minimization is not possible for this analysis.) It turns out that this can be the case, and we give the simplest example illustrating this, with just two rooms, although it is the case also for larger examples.

Let $n_{1}, n_{2}$ denote the number of patients in phases 1 and 2 of their treatment respectively, and let $n_{d}$ be the number of customers awaiting diagnostic results. Let $\mathbf{n}=\left(n_{1} n_{d} n_{2}\right)$ be the triple giving numbers in each state, with state space $\mathcal{S}=\left\{\mathbf{n}: n_{1}+n_{d}+n_{2}=N, n_{i} \geq 0\right\}$. Let $a(\mathbf{n}) \in\{0,1,2\}$ denote whether the doctor sees a patient in phase 1 or 2 of their service when the system is in state $\mathbf{n}$ or does not see a patient at all in state $\mathbf{n}$ (action 0 ), with $A=\{a(\mathbf{n}), \mathbf{n} \in S\}$ denoting a particular policy, and $\mathcal{A}$ the class of possible policies. The 0 action would typically only be taken in the state $(0 N 0)$ where all patients in rooms are awaiting the results of diagnostic tests. Note that the doctor only has a choice of which patient to see in those states where there is a patient at both phases of treatment. Let $\mathbf{e}_{i}$ denote the zero vector with a one in the $i^{\text {th }}$ entry. Let $\pi_{A}$ denote the stationary distribution under policy $A$.

Then the transition rates for this model are as follows:

$$
n \rightarrow \begin{cases}\mathbf{n}-\mathbf{e}_{1}+\mathbf{e}_{2} & \mu_{1}(1-p) \mathbb{1}\{a(\mathbf{n})=1\} \\ \mathbf{n}-\mathbf{e}_{2}+\mathbf{e}_{3} & n_{d} \mu_{d} \\ \mathbf{n}-\mathbf{e}_{3}+\mathbf{e}_{1} & \mu_{2} \mathbb{1}\{a(\mathbf{n})=2\}\end{cases}
$$

Note that while the system is in any state $\mathbf{n}$ such that $a(\mathbf{n})=1$, departures of patients requiring only one phase of treatment are occurring at rate $\mu_{1} p$. Hence the throughput under policy $A$ is given by

$$
\sum_{\mathbf{n}} \pi_{A}(\mathbf{n})\left(\mu_{1} p \mathbb{1}\{a(\mathbf{n})=1\}+\mu_{2} \mathbb{1}\{a(\mathbf{n})=2\}\right) .
$$

When $N=2$, there is only one state in which there is a choice between two types of patients, and that is state (101) with one patient in phase 1 of their treatment and the other in phase 2 .

Let policy 1 be the one where the doctor always treats the patient in phase one in state (101). Then the transition diagram of this system is depicted in Figure 11.1. Note that in this case the system never returns to state (200) once it has left it. For notational convenience, let $\tilde{\mu}_{1}=\mu_{1}(1-p)$. It is readily obtained 
Figure 11.1 Transition diagram for $N=2$ for policy 1 , with $\tilde{\mu}_{1}=\mu_{1}(1-p)$.

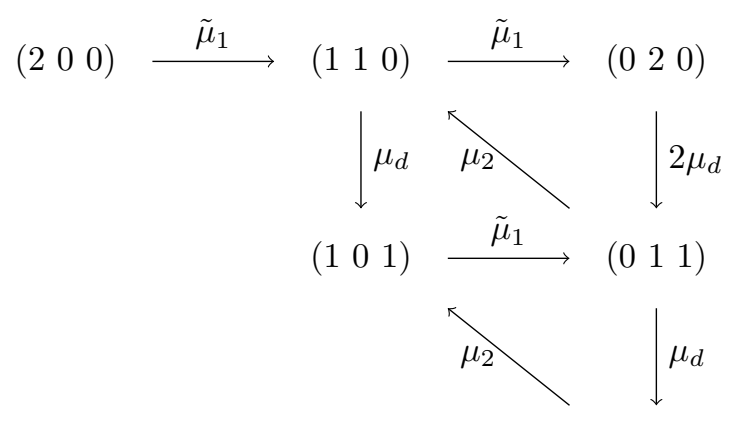

$\left(\begin{array}{lll}0 & 0 & 2\end{array}\right)$

that the equilibrium distribution is given by

$$
\begin{array}{lll}
\pi_{1}(020) & \propto 1 & \alpha:=\frac{\mu_{d}}{\mu_{1}(1-p)} \\
\pi_{1}(110) & \propto 2 \alpha, & \beta:=\frac{\mu_{d}}{\mu_{2}} \\
\pi_{1}(011) & \propto 2 \beta(1+\alpha), & \\
\pi_{1}(101) & \propto 2 \alpha(\alpha+\beta+\alpha \beta) & \\
\pi_{1}(002) & \propto 2 \beta^{2}(1+\alpha) . &
\end{array}
$$

The throughput of the system operating under policy 1 is given by

$$
\begin{aligned}
T_{1} & =\mu_{1} p\left[\pi_{1}(110)+\pi_{1}(101)\right]+\mu_{2}\left[\pi_{1}(011)+\pi_{1}(002)\right] \\
& =\frac{\mu_{1} p(2 \alpha+2 \alpha(\alpha+\beta+\alpha \beta))+\mu_{2} 2 \beta(1+\alpha+\beta(1+\alpha))}{1+2 \alpha(1+\alpha+\beta)+2 \beta(1+\alpha+\beta+\alpha \beta)} .
\end{aligned}
$$

Using $\alpha \mu_{1} p=\frac{\mu_{d} p}{1-p}$, this simplifies into

$$
\begin{aligned}
T_{1} & =\frac{2\left(\frac{\mu_{d} p}{1-p}+\mu_{d}\right)(1+\alpha)(1+\beta)}{1+2(\alpha+\beta)(1+\alpha)(1+\beta)} \\
& =\frac{2 \frac{\mu_{d}}{1-p}(1+\alpha)(1+\beta)}{1+2(\alpha+\beta)(1+\alpha)(1+\beta)} .
\end{aligned}
$$

In the following, we compare $T_{1}$ with the throughput of the system under policy 2 , i.e., when the doctor always treats the patient in phase 2 in state (101). If we would just remove the arrow from (101) to (011) and add an arrow from (101) to (200) to the system depicted in Figure 11.1, this would represent a system in which the doctor is allowed to preempt patients. Therefore, we extend state (101) with the location of the doctor, denoted by $(101, x)$ if the doctor is with the patient in phase $x, x=1,2$. The transition diagram for this system 
Figure 11.2 Transition diagram for $N=2$ for policy 2, without preemption of patients.

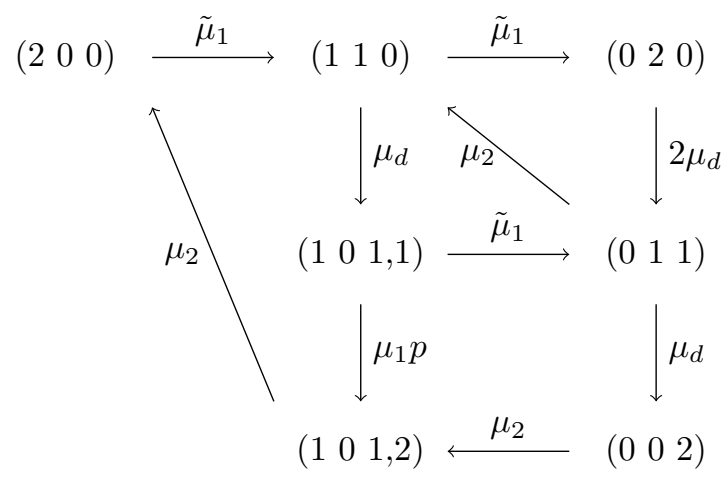

is depicted in Figure 11.2. For this system it is readily obtained that the equilibrium distribution is given by

$$
\begin{array}{ll}
\pi_{2}(020) & \propto 1 \\
\pi_{2}(110) & \propto 2 \alpha \\
\pi_{2}(011) & \propto \frac{2 \beta(1+\alpha(1-p))}{1+\beta} \\
\pi_{2}(101,1) & \propto 2 \alpha^{2}(1-p) \\
\pi_{2}(002) & \propto \frac{2 \beta^{2}(1+\alpha(1-p))}{1+\beta} \\
\pi_{2}(101,2) & \propto 2 \alpha \beta p+\frac{2 \beta^{2}(1+\alpha(1-p))}{1+\beta} \\
\pi_{2}(200) & \propto 2 \alpha^{2} p+\frac{2 \alpha \beta(1+\alpha(1-p))}{1+\beta} .
\end{array}
$$

The throughput of the system operating under policy 2 is given by

$$
\begin{aligned}
T_{2}=\mu_{1} p\left[\pi_{2}(200)+\pi_{2}(110)+\pi_{2}(101,1)\right] \\
+\mu_{2}\left[\pi_{2}(011)+\pi_{2}(002)+\pi_{2}(101,2)\right] \\
=\frac{2 \frac{\mu_{d}}{1-p}\left(1+\alpha+\frac{\beta}{1+\beta}(1+\alpha(1-p))\right.}{1+2(\alpha+\beta)\left(1+\alpha+\frac{\beta}{1+\beta}(1+\alpha(1-p))\right.} .
\end{aligned}
$$


With the expressions for $T_{1}$ and $T_{2}$ we formulate the following theorem.

Theorem 4. Consider a heavy traffic ED system with a single doctor treating patients who maximally require two phases of treatment, and between the two phases each patient joins an infinite server diagnosis queue while still occupying his treatment room. Let policy 1(2) imply that when the doctor always prioritizes patients in phase 1(2) of their treatment. Then, for an ED with two treatment rooms $(N=2)$ the throughput for policy 1 is higher than for policy 2 for all parameter settings, and policy 1 is in fact the optimal policy.

Proof. We have to show that $T_{1}>T_{2}$, and thus

$$
\frac{2 \frac{\mu_{d}}{1-p}(1+\alpha)(1+\beta)}{1+2(\alpha+\beta)(1+\alpha)(1+\beta)}>\frac{2 \frac{\mu_{d}}{1-p}\left(1+\alpha+\frac{\beta}{1+\beta}(1+\alpha(1-p))\right.}{1+2(\alpha+\beta)\left(1+\alpha+\frac{\beta}{1+\beta}(1+\alpha(1-p))\right.} .
$$

As all parameters are positive, this is equivalent to

$$
1+\alpha+\beta+\alpha \beta>1+\alpha+\frac{\beta}{1+\beta}(1+\alpha(1-p))
$$

Since $\alpha, \beta>0$, and $0 \leq p \leq 1, T_{1}>T_{2}$ holds for any parameter setting. This proves that giving priority to patient in phase one results in a higher throughput than giving priority to patients in phase two.

As the state space of this model is finite and the set of possible actions in each state is finite, according to proposition 4.4 .3 of [405] there exists a deterministic optimal policy for this system. For this system policies 1 and 2 are the only two possible deterministic policies, so policy 1 is in fact the optimal policy.

The results in Theorem 4 are only given for $N=2$. However, numerical investigations indicate that this result also holds for larger $N$.

From these extreme cases we may conclude that it is unlikely that there exists a policy that is optimal for all ED systems. As patients at an ED are temporarily unavailable for consultation due to diagnostic tests, doctors have to treat multiple patients in parallel to prevent that they are idle. However, when multiple doctors share the treatment rooms while all doctors are treating as many patients in parallel as possible, this may result in unbalanced treatment room assignments and may negatively affect the overall performance of the ED. In the following section we show that giving priority to new patients will not always result in the best performance for EDs, while this policy is optimal for extreme case three and appeared to result in the best performance for a more realistic ED system investigated in Chapter 10. Furthermore, is not a trivial task to optimally assign a doctor to each patient while taking differences between doctors, due to for example different specializations, into account. Three decisions are jointly optimized in the model presented in the following section: (1) how many patients each doctor should treat in parallel, (2) which patient a free doctor should treat next, and (3) which patients are treated by which doctors. 


\subsection{Model}

In this chapter, we minimize patients' LoS at the ED by optimizing both the patient/doctor assignments and the sequence in which patients are treated. We only consider the process at the ED after triage, when patients are labeled for a certain medical specialty, but are not yet assigned to one of the doctors. For time-varying arrival rates, the state space of a Markov decision problem quickly becomes too big for practically relevant scenarios. Therefore, we use a rolling horizon approach to maximize LoS-target compliance; each time one of the doctors becomes idle we invoke a mixed integer linear program (MIP) to decide which patient this doctor should treat next. The MIP consists of information from the current state of the ED, such as the number of patients present. The MIP solves a scenario in which we assume that each patient requires the maximum number of treatment phases for his type, which is close to a worst-case scenario. In the following, we define the sets, parameters, variables, constraints and the objective of the MIP.

Let $g \in \mathcal{G}=\left\{1, \ldots, N^{G}\right\}$ denote the doctors, and $p \in \mathcal{P}=\left\{1, \ldots, N^{P}\right\}$ the patients currently present at the ED. Each patient may require multiple phases of treatment, with phases $j \in \mathcal{J}=\left\{1, \ldots, N^{J}\right\}$. Here, phase 1 denotes the current phase of the patient's treatment, i.e., starts counting after the number of phases this patient has already completed. Furthermore, we introduce a set of integer event time points $n \in \mathcal{N}=\left\{1, \ldots, N^{N}\right\}$, in analogy with [248] and the 'Unit-Specific Event-Based Model' best tested in [446] for similar problems that are not dual resource-constrained. The event times are defined for each doctor and room separately.

The decision variables describing the optimal sequence of patients for each doctor after the optimization are denoted by $i_{g j p n}$, which equals one if phase $j$ of patient $p$ starts at event time $n$ with doctor $g$ and zero otherwise. For each doctor, each event time may be allocated to at most one event, which is ensured by the following constraint.

$$
\sum_{j \in \mathcal{J}, p \in \mathcal{P}} i_{g j p n} \leq 1 \quad \forall g \in \mathcal{G}, n \in \mathcal{N} .
$$

Each patient is treated by one doctor, and the patient is assigned to a doctor when the first phase of treatment commences. Therefore, we let $\mathcal{P}_{g}$ denote the subset of patients that may be treated by doctor $g$, which possibly includes new patients who are not yet assigned to a doctor. Additionally, we let $P_{g}^{C} \in \mathcal{P}$ denote the patient who is currently treated by doctor $g$. The following constraints assign the patient currently in service to the doctor.

$$
i_{g 1 p 1}=1 \quad \forall g \in \mathcal{G}, p=P_{g}^{C} .
$$

In order to prevent that doctors treat multiple patients in parallel, we introduce continuous variables $s_{g j p n}$, which denote the start time of phase $j$ of patient $p$ with doctor $g$ at event time $n$. Furthermore, let parameters $C_{g j p}$ and 
$T_{g j p}$ denote the average consultation time of phase $j$ and test time after phase $j$ of patient $p$ with doctor $g$, respectively. Note that by assuming average times the scenario solved by the MIP is slightly better than worst-case; $C$ and $T$ may be chosen differently, for example a certain percentile of the consultation and test time distributions. Constraints (11.5) ensure that phase $j+1$ of patient $p$ does not start before phase $j$ and the subsequent tests are completed. Constraints (11.6) ensure that the next event of doctor $g$ does not start before the current phase is completed. By constraints (11.7), (11.8), and (11.9) each patient's first phase is assigned to a doctor, and all consecutive phases are scheduled with the same doctor.

$$
\begin{aligned}
& \sum_{n^{\prime}>n \in \mathcal{N}} s_{g j p^{\prime} n^{\prime}}+M\left(1-\sum_{n^{\prime}>n \in \mathcal{N}} i_{g j p^{\prime} n^{\prime}}\right) \geq s_{g j p n}+\left(C_{g j p}+T_{g j p}\right) i_{g j p n} \\
& \forall g \in \mathcal{G}, j, j^{\prime}=j+1 \in \mathcal{J}, p \in \mathcal{P}, n \in \mathcal{N}, \\
& \sum_{j \in \mathcal{J}, p \in \mathcal{P}_{g}} s_{g j p n^{\prime}}+M\left(1-\sum_{j \in \mathcal{J}, p \in \mathcal{P}_{g}} i_{g j p n^{\prime}}\right) \geq \sum_{j \in \mathcal{J}, p \in \mathcal{P}_{g}} s_{g j p n}+C_{g j p} \cdot i_{g j p n} \\
& \forall g \in \mathcal{G}, n^{\prime}=n+1 \in \mathcal{N}, \\
& \sum_{g \in \mathcal{G}, n \in \mathcal{N}} i_{g 1 p n} \geq 1 \quad \forall p \in \mathcal{P}_{g}, \\
& C_{g j^{\prime} p} \cdot i_{g j p n} \leq M \sum_{n^{\prime}>n \in \mathcal{N}} i_{g j^{\prime} p n^{\prime}} \quad \forall g \in \mathcal{G}, j, j^{\prime}=j+1 \in \mathcal{J} \\
& p \in \mathcal{P}_{g}, n \in \mathcal{N} \\
& \sum_{j \in \mathcal{J}, g^{\prime} \neq g \in \mathcal{G}, n \in \mathcal{N}} i_{g^{\prime} j p n} \leq N^{P}\left(1-\sum_{n \in \mathcal{N}} i_{g 1 p n}\right) \quad \forall g \in \mathcal{G}, p \in \mathcal{P}_{g}
\end{aligned}
$$

Here, $M$ denotes a relatively large number, and $C_{g j^{\prime} p}$ in constraint (11.8) equals zero if phase $j$ is the last phase of patient $p$. Furthermore, we introduce $\mathcal{P}_{g}^{A}$, which denotes the subset of patients assigned to doctor $g$ who are currently away for tests, and parameter $R_{p}^{T}$ the remaining time until the test results of patient $p$ are available. The following constraints ensure that the patients currently away for tests are not visited by their doctor before the test results are available.

$$
\sum_{n \in \mathcal{N}} s_{g 1 p n} \geq R_{p} \quad \forall g \in \mathcal{G}, j \in \mathcal{P}_{g}^{A} .
$$

As the optimal patient schedule is, next to the number of doctors, also limited by the total number of rooms, we introduce the set of treatment rooms $\mathcal{R}=$ $\left\{1, \ldots, N^{R}\right\}$. Furthermore, indicator variable $i_{p r n}^{r}$ equals one if patient $p$ starts in room $r$ at event time $n$. The following constraints ensure that there is at most 
one patient assigned to each room for each event time.

$$
\sum_{p \in \mathcal{P}} i_{p r n}^{r} \leq 1 \quad \forall r \in \mathcal{R}, n \in \mathcal{N} .
$$

At the JBH, a treatment room is assigned to a patient from the start of the first phase until the end of the last phase of this patient. To this end, we introduce continuous variables $s_{p r n}^{r}$ and $f_{p r n}^{r}$, which denote the start and finish time of patient $p$ in room $r$ at event time $n$, respectively. Constraints (11.12) assign exactly one room to each patient. The variables $s^{r}$ and $f^{r}$ are set through constraints (11.13) and (11.14), and constraints (11.15) ensure that there is only one patient in each room at the time.

$$
\begin{array}{ll}
\sum_{r \in \mathcal{R}, n \in \mathcal{N}} i_{p r n}^{r}=1 & \forall p \in \mathcal{P} \\
\sum_{g \in \mathcal{G}, n \in \mathcal{N}} s_{g 1 p n} \geq \sum_{n \in \mathcal{N}, r \in \mathcal{R}} s_{p r n}^{r} & \forall p \in \mathcal{P}_{g} \\
\sum_{g \in \mathcal{G}, n^{\prime} \in \mathcal{N}} s_{g j p n^{\prime}}+C_{g j p} \cdot i_{g j p n^{\prime}} \leq \sum_{r \in \mathcal{R}, n \in \mathcal{N}} f_{p r n}^{r} & \forall j \in \mathcal{J}, p \in \mathcal{P} \\
\sum_{p \in \mathcal{P}} s_{p r n^{\prime}}^{r}+M\left(1-\sum_{p \in \mathcal{P}} i_{p r n^{\prime}}^{r}\right) \geq \sum_{p \in \mathcal{P}} f_{p r n}^{r} & \forall r \in \mathcal{R}, n, n^{\prime}=n+1 \in \mathcal{N} .
\end{array}
$$

In order to retrieve the MIP solution automatically, the schedule should start in event time point 1 . The following constraints ensure this for both the doctors and rooms.

$$
\begin{aligned}
\sum_{j \in \mathcal{J}, p \in \mathcal{P}} i_{g j p n^{\prime}} \leq \sum_{j \in \mathcal{J}, p \in \mathcal{P}} i_{g j p n} & \forall g \in \mathcal{G}, n, n^{\prime}=n+1 \in \mathcal{N}, \\
\sum_{p \in \mathcal{P}} i_{p r n^{\prime}}^{r} \leq \sum_{p \in \mathcal{P}} i_{p r n}^{r} & \forall r \in \mathcal{R}, n, n^{\prime}=n+1 \in \mathcal{N} .
\end{aligned}
$$

The main performance measure of the ED is the LoS norm compliance, i.e., the number of patients treated within the LoS norm. However, to prevent that the MIP discards patients for who the LoS norm is already exceeded, we minimize the sum of all lateness:

$$
\min \sum_{p \in \mathcal{P}, r \in \mathcal{R}, n \in \mathcal{N}} f_{p r n}^{r}-L_{p} .
$$

Here, $L_{p}$ denotes the remaining time until the LoS of patient $p$ exceeds the norm. Variables $i$ and $i^{r}$ are binary, and variables $s, s^{r}$ and $f^{r}$ are non-negative. The information we use from the MIP solution is which patient free doctor $g$ should see next, $i_{g 1 p 1}$, and the starting time of this patient, $s_{g 1 p 1}$. Note that the 
objective function allows for adding weights for certain patients, which may be useful if different patient types have different LoS targets. Furthermore, note that patient types are not explicitly modeled in the MIP; if a doctor is not qualified to treat a patient, the parameters $C$ and $T$ are set to zero for this doctor/patient combination. New patients who are not yet treated by a doctor, have non-zero $C_{g j p}$ values for all $g$ qualified to treat them, while for other patients only for their assigned doctor the $C_{g j p}$ values are non-zero. The MIP as formulated above is symmetric in the treatment rooms, so we assign all patients who are currently in a room at the ED to a specific room in the MIP.

In order to have practical relevance, the MIP should be solved within the order of seconds. As the only result we use from the MIP is which patient the available doctor will see next, and because all new patients of the same type are seen in order of arrival, we only incorporate one new patient of each type in the MIP to reduce the MIP's running time. For large instances, a further reduction of the running time could be achieved by interrupting the MIP solver before optimality is reached, for example when a sequence is found such that all LoS targets are met. However, for the case study considered in this chapter, interrupting the solver appeared unnecessary.

\subsection{Results}

This section provides the results of the rolling horizon approach. To this end, we introduce the case of the Jeroen Bosch Hospital (JBH), to which the approach is applied. We assess the performance of the MIP by invoking it from a discrete event simulation, and using the results again in the simulation. Therefore, this section provides an overview of the simulation model and its input. In order to assess the performance of our approach, for all scenarios we additionally simulate four priority policies: (1) an 'existing first' (EF) policy, in which a doctor always treats the patient with the longest LoS; (2) a 'new first' (NF) policy, in which the doctor always treats a patient from the waiting area with the longest LoS first, and otherwise treats the existing patient with the longest LoS; (3) a random (RND) policy, in which the doctor randomly chooses one of the available patients of his type; and (4) a 'next room' (NR) policy, in which the doctor visits the room with a higher index number (modulo the total number of rooms) with an available patient, and if this is an empty room the doctor may treat a new patient. In these policies all patients are still only treated by their own doctor.

\subsubsection{Case-study specific input}

To investigate the performance of our approach, we consider the ED of the JBH, which is also studied in Chapter 10. At this ED, three doctors with different specializations are present most of the day (from 7:00 until 23:00): a surgeon, an internist and an emergency physician. The surgeon and internist are specialized doctors, and can only treat patients arriving for their own medical specialty. The 
Table 11.1 Input scenario 1 for the JBH case, all times are averages and in minutes.

\begin{tabular}{ccccccccc}
\hline Patient & Physician $g$ & $C_{g 1 p}$ & $C_{g 2 p}$ & $C_{g 3 p}$ & $T_{g 1 p}$ & $T_{g 2 p}$ & $q_{g 1 p}$ & $q_{g 2 p}$ \\
\hline Type 1 & 1 & 15 & 10 & & 10 & & 0.1 & 1 \\
& 3 & 20 & 12 & 5 & 10 & 20 & 0.1 & 0.9 \\
\hline \multirow{2}{*}{ Type 2 } & 2 & 20 & 30 & 20 & 20 & 30 & 0.2 & 0.3 \\
& 3 & 30 & 22.5 & & 22.5 & & 0.4 & 1 \\
\hline
\end{tabular}

Table 11.2 Input scenario 2 for the JBH case, all times are averages and in minutes.

\begin{tabular}{ccccccccc}
\hline Patient & Physician & $C_{g 1 p}$ & $C_{g 2 p}$ & $C_{g 3 p}$ & $T_{g 1 p}$ & $T_{g 2 p}$ & $q_{g 1 p}$ & $q_{g 2 p}$ \\
\hline Type 1 & 1 & 5 & 5 & & 45 & & 0.1 & 1 \\
& 3 & 7 & 10 & 5 & 45 & 20 & 0.1 & 0.9 \\
\hline Type 2 & 2 & 15 & 10 & 15 & 45 & 45 & 0.2 & 0.3 \\
& 3 & 25 & 15 & & 90 & & 0.4 & 1 \\
\hline
\end{tabular}

emergency physician is specially trained for providing urgent care to a wide range of diagnoses. The ED is equipped with eight treatment rooms, which may be used by all doctors.

For the ease of presentation, in this chapter we assume that all patients are from non-emergency triage classes, but, as mentioned before, different urgency classes can readily be incorporated in the objective function of the MIP. Additionally, we only consider surgical or internal medicine patients, and both types may be treated by the specialized doctor or the emergency physician. Furthermore, we introduce parameter $q_{g j p}$ as the probability that patient $p$ leaves the ED after phase $j$ if he is treated by doctor $g$. We do not have data on the distribution of the number of phases of treatment and (distribution of) the duration of each phase. We limit the number of phases to three in all investigated scenarios, but the model is readily extended to incorporate more phases.

We investigate a realistic scenario (labeled 'scenario 1') in which the type 1 patients and doctor are surgical, the type 2 patients and doctor are internal medicine, and doctor 3 is the emergency physician; all detailed input is presented in Table 11.1. In this scenario, surgical patients generally require one (series of) diagnostic test(s) and we assume that patients treated by the emergency physician have slightly longer treatment times compared to patients treated by the surgeon. Additionally, the internist generally requires more phases of treatment compared to the emergency physician, which is in line with the results of [274]. Additionally, we investigate in scenario 2 an ED system in which the time for obtaining diagnostic test results is relatively long, which is presented in Table 11.2.

The discrete event simulation used for this chapter is similar to the one used in Chapter 10. Patients for both specialties arrive according to a time-varying Poisson process, for which the rates are derived from JBH data (see Figure 10.2). For both scenarios we investigate two cases: (a) a case with five treatment rooms and only the non-urgent surgical and internal medicine patients, and (b) a case with eight treatment rooms and the arrival rates scaled up so together they equal 
the total arrival rate at the ED of the JBH. We denote scenario 1 for case (a) by 'scenario 1(a)'.

The simulation is implemented in $\mathrm{C}++$. We simulate 120 days 30 times, and use common random numbers [294] for a fair comparison between the different scheduling policies. In order to apply common random numbers, we limit the total number of patients present at the ED to 40; preliminary results indicated that the blocking probability is negligible in this case. Recall that in the MIP each patient stays the maximum number of phases in the system, and each phase of treatment or tests is set to take the average time. In the simulation, each phase of treatment and tests is exponentially distributed, for which the average times are equal to the averages used in the MIP.

The MIP is implemented in AIMMS ${ }^{\circledR}$, and operated from the simulation with AIMMS SDK. The MIP is only solved when a doctor has a choice between multiple patients, i.e., if there is a free room and at least one new and one existing patient the doctor may treat. We assume that practitioners at the ED can estimate the remaining test times accurately, so we use the actual remaining test times in parameter $R_{p}$. The remaining time in a treatment phase is assumed to be equal to the average time in that phase. The parameter for the number of patients in the system, $N^{P}$, equals the number of occupied rooms plus two (one patient of each type). For each patient we store the arrival time at the ED, which is used to determine $L o S_{p}$ each time the MIP is invoked. We assume that each patient has a LoS norm of three hours, which holds for all Dutch hospitals. Parameter $M$ is set at two times the LoS target, and the number of event time points equals the maximum number of treatment phases a doctor may possibly perform. Therefore, $N^{N}$ depends on the number of patients each doctor is treating and in which phase these patients are. In case the model is infeasible, $M$ and $N^{N}$ are increased with the LoS target and one, respectively, and the model is solved again. We define all room-related variables only for half of the event time points to reduce the computation time. The MIP is always solved to optimality within one minute, and often within a few seconds, for all investigated scenarios.

\subsubsection{Performance of the policies}

From the simulation runs it appears that the performance of the MIP policy is close to the performance of the EF policy, see Figures 11.3 and 11.4 for the results of a single run of the simulation for scenario $1(\mathrm{~b})$. In these figures $(h)$ denotes hours. The performance of the NF policy is significantly worse compared to the MIP and EF policies for all scenarios. The performance of the other policies is slightly worse compared to the performance of the MIP and EF policies, for all scenarios. From Figure 11.4 it appears that patients of type 2 treated by doctor 2 have relatively long LoSs, compared to the other patients, and for these patients the MIP policy appears to result in the best performance. The EF and NR policy result in similar LoS-norm compliance, which appears to be poor in this scenario. For doctors 1 and 3 treating type 1 patients, the probability that the 
LoS target is exceeded equals $20.4 \%$ and $30.6 \%$, respectively. Type 2 patients have even higher probability of exceeding the LoS target: almost $34.8 \%$ when they are treated by doctor 2, and $29.7 \%$ when treated by doctor 3 . All other scenarios have a significantly higher LoS-target compliance, but are still lower than the compliance the JBH aims for.

Figure 11.3 Cumulative LoS density for type 1 patients treated by doctor 1 (left) and 3 (right) in scenario $1(\mathrm{~b})$.
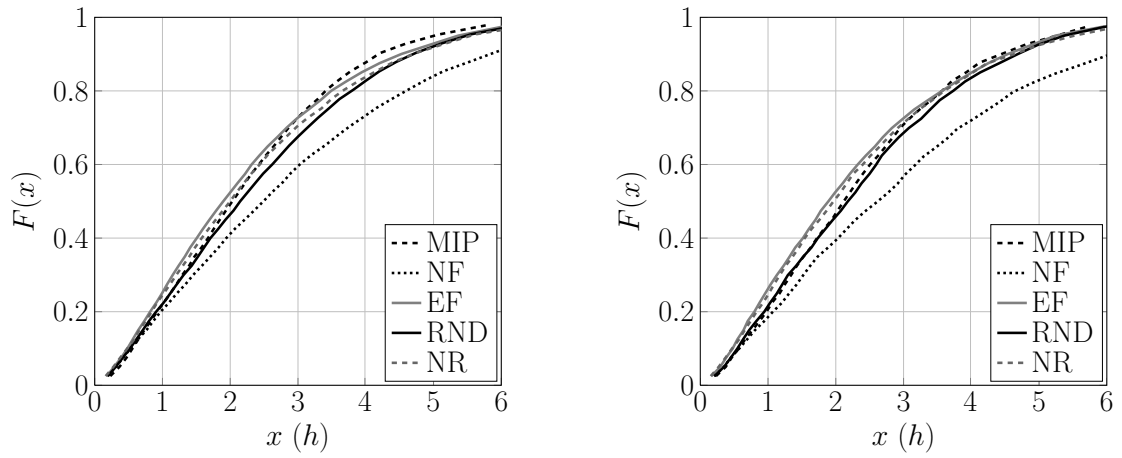

Figure 11.4 Cumulative LoS density for type 2 patients treated by doctor 2 (left) and 3 (right) in scenario 1 (b).
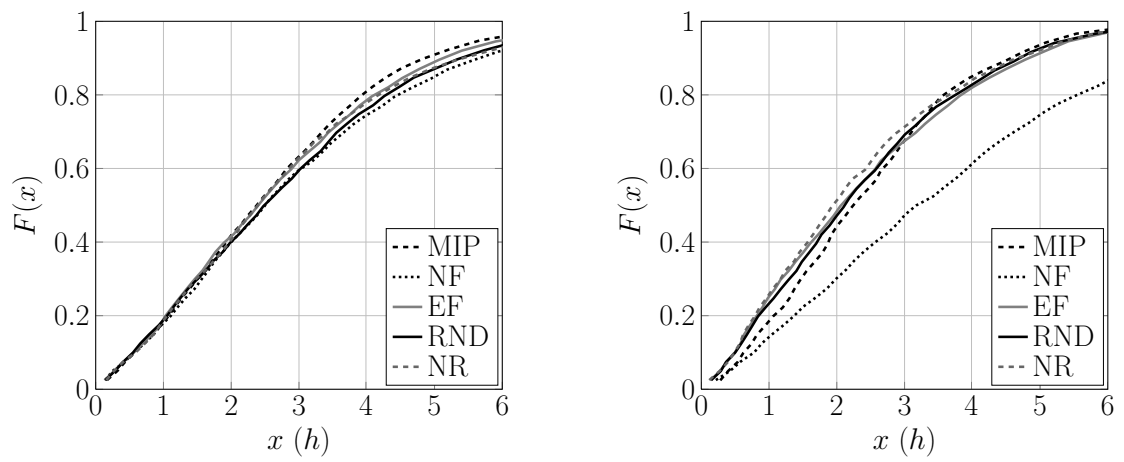

The average LoS and waiting time for all investigated scenarios are depicted in Figure 11.5, in which ' $\mathbb{E}\left[L o S_{31}\right]$ ' denotes the average LoS for type 1 patients treated by doctor 3 and ' $\mathbb{E}\left[W_{1}\right]$ ' denotes the average waiting time for type 1 patients. It appears that the LoS of type 2 patients consists of relatively short waiting time, while type 1 patients have relatively long waiting times.

It appears from the simulation results that the policy does not have a significant effect on the average LoS or waiting time, except for the NF policy; the performance of the NF policy is relatively poor. Moreover, the Los target compliance is significantly better for all scenarios with the MIP and EF policies compared to the NF policy. 
Figure 11.5 Average performance measures with $95 \%$ confidence intervals for the MIP policy.

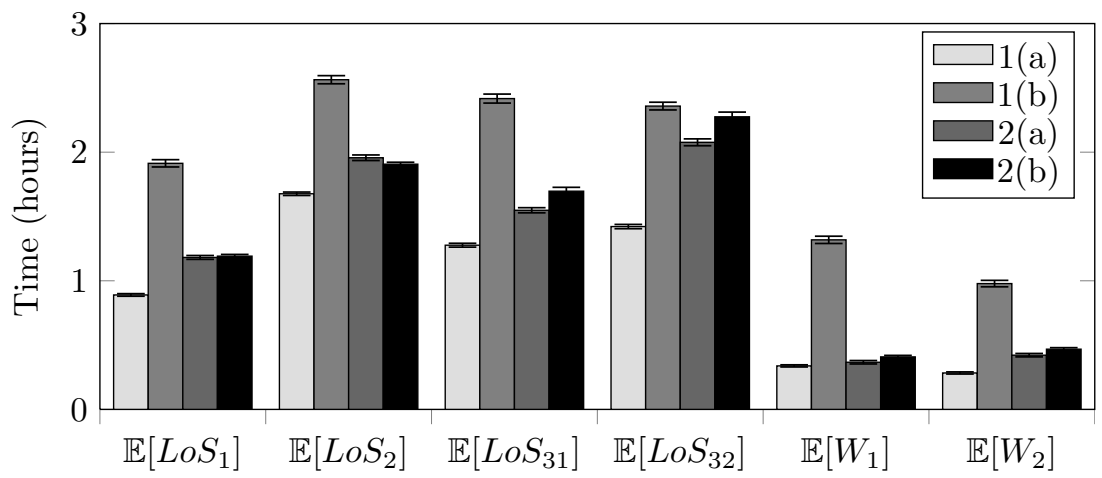

\subsubsection{The best policy}

From the numerical results of scenarios 2(a) and 2(b) it appears that the performance of all policies is close. For scenarios 1(a) and 1(b) the performance of the MIP and EF policies is close, and the other policies perform significantly worse. For all scenarios except 1(b), the performance of the NR policy is the third best, and still quite close to the performance of the EF and MIP policies. In order to compare the policies, we analyze the decisions based on the MIP solution for the same simulation run as in the previous subsection.

For scenarios 1(a) and 1(b) the MIP solution predominantly prescribes to prioritize existing patients, see Tables 11.3 and 11.4. Physician 2 requires the MIP for decision making the least, but is relatively often advised to give priority to new patients or do nothing. The MIP is called 1337 times in 120 days in scenario 1(a), and 7761 times in scenario 1(b). In scenario 1(a) over 7500 patients were treated in total in this run, and over $70 \%$ of the patients were treated by the specialized doctor for both types. In scenario 1(b) 12179 patients were treated, of which $66 \%$ of the type 1 patients and $73 \%$ of the type 2 patients were treated by the specialized doctor. The states in which the MIP decides not to prioritize existing patients do not show a clear distinction from other states, so no general rule could be obtained from this scenario.

Table 11.3 Specification of the decisions from the MIP in scenario 1(a).

\begin{tabular}{lccccc}
\hline Decision & Patient type & Physician 1 & Physician 2 & Physician 3 & Overall \\
\hline Existing patient & Type 1 & $99.0 \%$ & & $80.0 \%$ & $78.4 \%$ \\
& Type 2 & & $94.9 \%$ & $18.3 \%$ & $19.8 \%$ \\
New patient & & \multirow{2}{*}{$1.0 \%$} & $3.6 \%$ & & $1.0 \%$ \\
Do nothing & & & $1.5 \%$ & $1.6 \%$ & $0.7 \%$ \\
\hline Overall & & $53.4 \%$ & $14.7 \%$ & $31.9 \%$ & $100.0 \%$ \\
\hline
\end{tabular}

Although the performances of the EF and MIP policies are also similar for scenario 2(a), in scenario 2(a) the MIP policy prioritizes new patients relatively 
Table 11.4 Specification of the decisions from the MIP in scenario 1(b).

\begin{tabular}{lccccc}
\hline Decision & Patient type & Physician 1 & Physician 2 & Physician 3 & Overall \\
\hline Existing patient & Type 1 & $99.2 \%$ & & $83.1 \%$ & $70.9 \%$ \\
& Type 2 & & $89.1 \%$ & $14.4 \%$ & $25.4 \%$ \\
New patient & & $0.8 \%$ & $5.4 \%$ & & $1.6 \%$ \\
Do nothing & & & $5.6 \%$ & $2.4 \%$ & $2.1 \%$ \\
\hline Overall & & $44.1 \%$ & $23.2 \%$ & $32.7 \%$ & $100.0 \%$ \\
\hline
\end{tabular}

often for doctor 1 compared to scenario 1, see Table 11.5. Additionally, doctor 3 treats more type 2 patients compared to scenario 1 . The decisions of the MIP policy for scenario 2(b) are presented in Table 11.6, from which it appears that doctor 2 should almost always treat existing patients first. In scenario 2(a) the MIP is solved 1977 times, while for scenario 2(b) only 527 times.

Table 11.5 Specification of the decisions from the MIP in scenario 2(a).

\begin{tabular}{lccccc}
\hline Decision type & Patient type & Physician 1 & Physician 2 & Physician 3 & Overall \\
\hline Existing patient & Type 1 & $67.3 \%$ & & $71.5 \%$ & $54.0 \%$ \\
& Type 2 & & $88.9 \%$ & $27.1 \%$ & $23.6 \%$ \\
New patient & & $32.7 \%$ & $10.2 \%$ & & $22.0 \%$ \\
Do nothing & & & $1.0 \%$ & $1.4 \%$ & $0.5 \%$ \\
\hline Overall & & $60.6 \%$ & $20.9 \%$ & $18.5 \%$ & $100.0 \%$ \\
\hline
\end{tabular}

Table 11.6 Specification of the decisions from the MIP in scenario 2(b).

\begin{tabular}{lccccc}
\hline Decision type & Patient type & Physician 1 & Physician 2 & Physician 3 & Overall \\
\hline Existing patient & Type 1 & $77.0 \%$ & & $67.2 \%$ & $57.7 \%$ \\
& Type 2 & & $98.9 \%$ & $32.8 \%$ & $26.9 \%$ \\
New patient & & $23.0 \%$ & $1.6 \%$ & & $15.4 \%$ \\
Do nothing & & & & \\
\hline Overall & & & & & \\
\hline
\end{tabular}

Concluding, it appears that in the investigated realistic ED systems, the EF policy performs similar to the MIP policy. As the EF policy is a simple heuristic, it would be much easier to implement in practice compared to the MIP policy. Furthermore, the numerical results indicate that the NR policy performs relatively good for ED systems with a small number of rooms, and the NF policy (which appeared superior to the EF policy in the heavy traffic analysis of a simplified system, Theorem 4) performs the worst of all investigated policies. From all investigated policies and scenarios it appears that the LoS-target compliance is lower than the aim of the $\mathrm{JBH}$; the hospital has already initiated other projects to reduce patients' LoS at the ED. 


\subsection{Conclusion}

In this chapter we investigated a dual resource constrained system with multiple distinct servers, which is found at EDs. In this system, patients require multiple phases of treatment and are unavailable for treatment in between of these phases. Physicians follow a policy that prescribes the sequence in which the patients are treated. From the investigated extreme cases we may conclude that it is unlikely that there exists a policy that is optimal for all ED systems. We optimized the priority policy of each doctor and the patient-doctor assignments in each state of the ED system using a mixed integer programming model in a rolling-horizon approach, in which we incorporated time-varying arrival rates and that each phase of treatment may have different characteristics that depend on the type of doctor and patient. Numerical experiments indicated that our policy performs similar to the policy that gives priority to the patient with the longest LoS. As this heuristic policy is easy and performs well, these results are of significant practical relevance.

Although the ED investigated in Chapter 10 is similar to the one considered in this chapter, the conclusion on which policy results in the best performance is exactly opposite. These opposite results could be caused by the assumption in Chapter 10 that as soon as there is more than one patient in server, the diagnostic test times are negligible. However, the heavy traffic analysis for Theorem 4 indicates that this is not a crucial assumption for these results to hold. From the results of this chapter and Chapter 10 we may conclude that the best policy depends on the parameters of the ED system, but it is unclear which parameters are most important, if any, for the system to switch between giving priority to new or to existing patients. Therefore, further research will address this question.

The optimization model does not take stochasticity into account, and assumes that every patient stays in the system for the maximum number of phases. The formulation of the model may be extended to incorporate that patients may stay for less than the maximum number of phases. However, this extension will require significantly more variables and thus solving time, implying that additional measures should be taken to reduce the model's solving time.

At the hospital in this case study patients may await their test results in their treatment room, whereas in other hospitals patients may have to wait in a waiting area. The policy that patients occupy the room throughout their entire length of stay generally restricts the number of patients that can be treated in parallel. It would be interesting for future research to investigate the performance of the ED without this restriction. Furthermore, in this chapter we assumed that the switch-over times for a doctor to go from one room to another are negligible, but this may not be a realistic assumption for all EDs. Our optimization model is readily extended to incorporate switch-over times, and it may be interesting to investigate how these times affect the ED's performance. 


\section{2}

\section{Conclusion and outlook}

Healthcare expenditures have been rapidly rising in the last 50 years, and there is much potential to improve logistics and logistical support in hospitals. Despite the vast amount of academic papers on this topic and the large potential of Operations Research (OR) methodologies, it appears that the actual implementation of OR models and/or results in healthcare practice is rare. This thesis aims to bridge the gap between theory and practice, as it displays both research that focuses on theoretical results and implementation-oriented case studies. Furthermore, we elaborate on the experienced problems with implementing research results, and provide several factors for successful implementation. Although most methodologies in this thesis are designed for hospital case studies, the methodologies and/or results may be generalized to other hospitals and application areas. This chapter provides the main conclusions of this thesis, and closes with an outlook on OR in healthcare.

The literature on OR methodologies is extensive, as Chapters 2 and 4 highlight for OR models applied specifically to hospital wards and online appointment scheduling, respectively. Both chapters provide a structured overview of the rapidly growing field of OR literature, aiming both to guide interested readers to existing methods, and to identify research opportunities. Additionally, by focusing on implemented OR research, the review in Chapter 2 aims to reduce the gap between theoretic research and practical implementations. By not restricting to healthcare applications, Chapter 4 aims to stimulate mutual interchange of research results from different application areas.

Hospitals typically work with appointment systems for their elective patients. In Chapter 5 we show for a simple queueing system that offering patients appointments instead of letting them walk-in may significantly affect the system's accessibility. Depending on the parameters of the system, appointments either increase or decrease the blocking probability. Additionally, from Chapter 7 it appears that the performance obtained with treating as many patients as medically possible on a walk-in basis is comparable to the performance of the best found appointment rule. As appointment systems require more organizational efforts, for many hospital departments it would be valuable to investigate the possibilities and benefits of evolving into a walk-in system.

Patient appointment schedules determine to a large extent patients' access 
and waiting times, and doctor idle and overtime. In Chapter 6 we showed that minor changes to the appointment schedule may already have significant impact. Appointments for each patient type were clustered in the schedule, but it appeared that spreading the patient types over the schedule reduced waiting times significantly. This was due to the different stochastic capacity requirements for each patient type. Also in Chapter 7 we obtained appointment rules that are both promising to reduce overtime and easy to implement, but for a system with combined appointments and walk-in patients. Key to these solutions was to schedule appointments in the times that have fewer arrivals of walk-in patients. However, for this case study it appeared that in order to prevent exceptionally busy times, the clinic will have to investigate other interventions, such as offering walk-in patients an appointment at a later time.

Patient appointment schedules are based on doctors' schedules. Healthcare practitioners are often aware of variability and seasonality in the demand for healthcare, but typically also the supply of care varies significantly. Physicians may not be available for outpatient consultations due to their many other tasks, such as ward or emergency department (ED) duties, performing surgeries, and education. Creating good doctor schedules is therefore a difficult task. In Chapter 8 we provided pragmatic solutions to this task, which significantly reduce the scheduler's time spend on creating the schedules and improve both patients' access time and doctors' work satisfaction. Next to an improved static doctor schedule, we showed in Chapter 9 that dynamically allocating capacity increases both the efficiency and performance of an outpatient clinic. In this scenario, the supply of care is linked to the varying demand, reducing wasted capacity while improving accessibility for patients.

In hospitals patients typically arrive either via the outpatient clinic or via the ED. EDs often experience severe overcrowding, which may put patient lives at risk. Typically, doctors at EDs use multiple rooms in parallel; while one patient awaits test results in a treatment room, the doctor visits other patients. The assignment of rooms among the doctors is often unbalanced, which affects the blocking probability, waiting time and length of stay of patients. In Chapter 10 we investigate different room assignment policies and working routines of the ED doctors, and in Chapter 11 we optimize these policies and routines. Depending on the characteristics of the ED, doctors should either give priority to new patients or to patients they have already treated before. Based on the results of Chapters 10 and 11 , we conclude that it is unlikely that there exists a policy that is optimal for all ED systems.

The advantage of OR models for healthcare is that possible interventions can be evaluated in a safe virtual environment, reducing the risk of piloting an intervention that appears to be counter-productive. Despite this, from Chapter 2 it appears that reported practical implementations of OR models are sparse. We are convinced that this final and for practitioners most important phase of an OR project should receive more attention both during OR projects and in OR literature. In Section 2.7 we provide a list of factors that improve implementation 
likelihood, which is consistent with experiences with the implemented research reported in Chapters 3 and 6 . We are confidently optimistic that the provided illustrations of applied research in this thesis inspire both researchers and healthcare professionals in exploring the possibilities and opportunities OR offers for healthcare, and making the extra step towards improving healthcare logistics in practice.

One of the reasons why OR research often lacks implementation in practice, is that the developed models are too demarcated and/or the results too complicated to be of practical relevance. Academics generally develop new theories and models, while for practitioners simple existing methods may already result in a significant improvement. From our experiences in the collaborating hospital, especially with the research reported in Chapter 6, it appears that hidden capacity problems are already discovered just because practitioners take the time to discuss the process with each other. Therefore, an OR researcher may also contribute to improvements in (healthcare) practice by temporarily shifting practitioners' focus from an ad hoc to a more holistic viewpoint. A lack of medical knowledge may actually help an OR researcher in healthcare, as logistical irregularities in care processes will stand out more, and practitioners will 'think out-of-the-box' when medically infeasible but logistically sensible solutions are proposed.

In the near future the focus area of this thesis, appointment scheduling, will become more important. A recent trend in healthcare is the increasing number of outpatient procedures. Due to technological advancements, for many procedures it is no longer medically necessary for patients to stay over night. Already the number of outpatient procedures is $50 \%$ of the total number of procedures [236]. As a consequence, the number of inpatient beds in hospital will reduce, and more outpatient beds will be required. Outpatient care is short and scheduled in advance, leaving many possibilities for optimizing patient admission scheduling.

Dutch hospitals have recently begun or are about to introduce digital patient records, often referred to as 'electronic health record' or 'electronic medical record'. Digital records add significantly to the already large healthcare data collection and have the potential to support both logistical and medical practices. Supercomputers can already advise doctors on the most promising treatments [131]. Logistical inefficiencies may be detected early as management information on important performance indicators can be obtained automatically real-time. In such cases, more reliable data is available for research purposes, which may improve both medical and logistical performances. However, the quantities of generated data are such that keeping a holistic viewpoint or extracting specific information might become a highly specialized task. Software companies can to a large extent support in these issues, but healthcare managers and researchers will have to be educated as well. For OR researchers in healthcare, big data theory will become more important. Furthermore, with this data the models may mimic reality better, and thus provide solutions with higher 
practical relevance.

Healthcare has become more patient-centered, which forces different medical departments to align their activities. Additionally, patient co-morbidity is increasing, which also forces several medical specialties to increase their cooperation and communication with other specialisms. Moreover, a recent trend is to organize healthcare more into specialized centers, which typically increases patient travel times. As a consequence, offering multiple appointments on one day is increasingly important. Research has to adapt, and be aimed more at scheduling and optimizing care pathways and the interaction between departments, instead of focused on single departments. Even without these developments, hospital departments already have strongly inter-related inflow of patients; patient arrivals at emergency departments and outpatient clinics will determine arrivals at the operation theater and hospital wards. However, researchers appear to focus on optimizing single departments [492], and often even on single resource systems in which patients require only one appointment (see Chapter 4). Ultimately, involving care providers outside the hospital, such as nursing homes, in the optimization would further reduce delays in patients' treatments.

In the near future, technological advances in point-of-care testing and telemedicine will increase the possibilities for diagnosing or treating more patients from their homes. Furthermore, robots may take over supportive tasks, or even perform surgery or judge diagnostic test results. As a consequence, healthcare delivery and healthcare logistics will change, opening up opportunities for OR researchers. Some examples of new logistical challenges are: optimizing the sequence of surgical activities, optimizing material and/or patient transportation in hospitals, and developing follow-up strategies that prescribe when patients are required to visit the hospital and when they may be treated from their homes. Additionally, OR methodologies may assess the costs and benefits of letting robots take over practitioners' tasks.

In summary, this thesis provides both theoretical and pragmatic applications of OR methodologies that support logistical decision making in hospitals. Optimizing the efficiency of healthcare logistics often improves both patient-friendliness and quality of care through better accessibility and alignment of the appointments, and may provide the hospital with the possibility to treat more patients with the same capacity. We focus on inventive mathematical solutions with a human touch, by incorporating both patient and doctor preferences in our approaches. With this thesis we intend to bring theory and practice closer together, so academics and practitioners can join forces in the continuous improvement of the efficiency of healthcare logistics. 


\section{Bibliography}

[1] S. Adabi, A. Movaghar, and A.M. Rahmani. Bi-level fuzzy based advanced reservation of cloud workflow applications on distributed grid resources. The Journal of Supercomputing, 67(1):175-218, 2014.

[2] I. Adan, J. Bekkers, N. Dellaert, J. Jeunet, and J. Vissers. Improving operational effectiveness of tactical master plans for emergency and elective patients under stochastic demand and capacitated resources. European Journal of Operational Research, 213(1):290-308, 2011.

[3] I.J.B.F. Adan and J.M.H. Vissers. Patient mix optimisation in hospital admission planning: a case study. International Journal of Operations 86 Production Management, 22(4):445-461, 2002.

[4] J.D. Adler and P.B. Mirchandani. Online routing and battery reservations for electric vehicles with swappable batteries. Transportation Research Part B, 70: 285-302, 2014.

[5] A. Afzal, A.S. McGough, and J. Darlington. Capacity planning and scheduling in grid computing environments. Future Generation Computer Systems, 24(5): 404-414, 2008.

[6] N. Agatz, A. Campbell, M. Fleischmann, and M. Savelsbergh. Time slot management in attended home delivery. Transportation Science, 45(3):435-449, 2011.

[7] K. Aida and H. Casanova. Scheduling mixed-parallel applications with advance reservations. Cluster Computing, 12(2):205-220, 2009.

[8] E. Akcali, J.C. Murray, and C. Lin. A network flow approach to optimizing hospital bed capacity decisions. Health Care Management Science, 9(4):391-404, 2006 .

[9] Y. Akçay, A. Balakrishnan, and S.H. Xu. Dynamic assignment of flexible service resources. Production and Operations Management, 19(3):279-304, 2010.

[10] G. Akin, J.S. Ivy, T.R. Huschka, T.R. Rohleder, and Y.N. Marmor. Capacity management and patient scheduling in an outpatient clinic using discrete event simulation. In Proceedings of the Winter Simulation Conference, pages 2215-2226, Washington, USA, 2013.

[11] R. Akkerman and M. Knip. Reallocation of beds to reduce waiting time for cardiac surgery. Health Care Management Science, 7(2):119-126, 2004.

[12] S. Albers. Recent advances for a classical scheduling problem. In F.V. Fomin, R. Freivalds, M. Kwiatkowska, and D. Peleg, editors, Automata, Languages, and Programming, volume 7966 of Lecture Notes in Computer Science, pages 4-14. Springer-Verlag, Berlin Heidelberg, Germany, 2013. 
[13] S.L. Albin, J. Barrett, D. Ito, and J.E. Mueller. A queueing network analysis of a health center. Queueing Systems, 7(1):51-61, 1990.

[14] G.F. Anderson, P.S. Hussey, B.K. Frogner, and H.R. Waters. Health spending in the united states and the rest of the industrialized world. Health Affairs, 24(4): 903-914, 2005.

[15] K. Anderson, B. Zheng, S.W. Yoon, and M.T. Khasawneh. An analysis of overlapping appointment scheduling model in an outpatient clinic. Operations Research for Health Care, 4:5-14, 2015.

[16] E. Angelelli, M.G. Speranza, J. Szoldatics, and Z. Tuza. Geometric representation for semi on-line scheduling on uniform processors. Optimization Methods and Software, 25(3):421-428, 2010.

[17] J.A. Araúzo, J. Pajares, and A. Lopez-Paredes. Simulating the dynamic scheduling of project portfolios. Simulation Modelling Practice and Theory, 18(10):14281441, 2010.

[18] M. Armony and C. Maglaras. Contact centers with a call-back option and realtime delay information. Operations Research, 52(4):527-545, 2004.

[19] A. Armstrong and J. Meissner. Railway revenue management: overview and models. Working paper (available at www.meiss.com), Lancaster University Management School, Lancaster, UK, 2010.

[20] O.M. Ashour and G.E. Okudan Kremer. Dynamic patient grouping and prioritization: a new approach to emergency department flow improvement. Health Care Management Science, pages 1-14, 2014.

[21] B. Ata and T.L. Olsen. Near-optimal dynamic lead-time quotation and scheduling under convex-concave customer delay costs. Operations Research, 57(3):753-768, 2009 .

[22] V. Auletta, R. De Prisco, P. Penna, and G. Persiano. On designing truthful mechanisms for online scheduling. Theoretical Computer Science, 410(36):3348$3356,2009$.

[23] N. Ayvaz and W.T. Huh. Allocation of hospital capacity to multiple types of patients. Journal of Revenue and Pricing Management, 9(5):386-398, 2010.

[24] U.Th. Bachmann, M.M. Halldórsson, and H. Shachnai. Online selection of intervals and $t$-intervals. Information and Computation, 233:1-11, 2013.

[25] A. Bagust, M. Place, and J.W. Posnett. Dynamics of bed use in accommodating emergency admissions: stochastic simulation model. BMJ, 319(7203):155-158, 1999.

[26] N.T.J. Bailey. A study of queues and appointment systems in hospital out-patient departments, with special reference to waiting-times. Journal of the Royal Statistical Society. Series B (Methodological), 14(2):185-199, 1952. 
[27] H. Balasubramanian, A. Muriel, and L. Wang. The impact of provider flexibility and capacity allocation on the performance of primary care practices. Flexible Services and Manufacturing Journal, 24(4):422-447, 2012.

[28] H. Balasubramanian, S. Biehl, L. Dai, and A. Muriel. Dynamic allocation of same-day requests in multi-physician primary care practices in the presence of prescheduled appointments. Health Care Management Science, 17(1):31-48, 2014.

[29] C. Banditori, P. Cappanera, and F. Visintin. A combined optimization-simulation approach to the master surgical scheduling problem. IMA Journal of Management Mathematics, 24(2):155-187, 2013.

[30] A. Bar-Noy, J.A. Garay, and A. Herzberg. Sharing video on demand. Discrete Applied Mathematics, 129(1):3-30, 2003.

[31] C. Barnhart, P. Belobaba, and A.R. Odoni. Applications of operations research in the air transport industry. Transportation Science, 37(4):368-391, 2003.

[32] Y. Baryshnikov, E.G. Coffman Jr., and P. Jelenković. Space filling and depletion. Journal of Applied Probability, 41(3):691-702, 2004.

[33] C. Barz and R. Kolisch. Hierarchical multi-skill resource assignment in the telecommunications industry. Production and Operations Management, 23(3): 489-503, 2014.

[34] C. Barz and K. Rajaram. Elective patient admission and scheduling under multiple resource constraints. Production and Operations Management, 24(12):19071930, 2015.

[35] H. Beaulieu, J.A. Ferland, B. Gendron, and M. Philippe. A mathematical programming approach for scheduling physicians in emergency room. Health Care Management Science, 3:193-200, 2000.

[36] R. Bekker and A.M. de Bruin. Time-dependent analysis for refused admissions inclinical wards. Annals of Operations Research, 178(1):45-65, 2010.

[37] R. Bekker and P.M. Koeleman. Scheduling admissions and reducing variability in bed demand. Health Care Management Science, 14(3):237-249, 2011.

[38] J. Beliën and E. Demeulemeester. Building cyclic master surgery schedules with leveled resulting bed occupancy. European Journal of Operational Research, 176 (2):1185-1204, 2007.

[39] J. Beliën, E. Demeulemeester, and B. Cardoen. A decision support system for cyclic master surgery scheduling with multiple objectives. Journal of Scheduling, 12(2):147-161, 2009.

[40] R. Ben Bachouch, A. Guinet, and S. Hajri-Gabouj. An integer linear model for hospital bed planning. International Journal of Production Economics, 140(2): 833-843, 2012.

[41] A.R. Bennett and A.L. Erera. Dynamic periodic fixed appointment scheduling for home health. IIE Transactions on Healthcare Systems Engineering, 1(1):6-19, 2011. 
[42] G. Berbeglia, J.F. Cordeau, and G. Laporte. Dynamic pickup and delivery problems. European Journal of Operational Research, 202(1):8-15, 2010.

[43] B. Berg and B.T. Denton. Appointment planning and scheduling in outpatient procedure centers. In R. Hall, editor, Handbook of Healthcare System Scheduling, volume 168 of International Series in Operations Research 83 Management Science, chapter 6, pages 131-154. Springer Science+Business Media, Dordrecht, the Netherlands, 2012.

[44] T.J. Best, B. Sandikci, D. Eisenstein, and D. Meltzer. Managing hospital bed capacity through partitioning care into focused wings. Manufacturing \& Service Operations Management, 17(2):157-176, May 2015.

[45] Hans-Georg Beyer and Bernhard Sendhoff. Robust optimization - a comprehensive survey. Computer Methods in Applied Mechanics and Engineering, 196 (33-34):3190 - 3218, 2007. ISSN 0045-7825.

[46] S. Bhulai and G. Koole. A queueing model for call blending in call centers. IEEE Transactions on Automatic Control, 48(8):1434-1438, 2003.

[47] I.A. Bikker, N. Kortbeek, R.M. van Os, and R.J. Boucherie. Reducing access times for radiation treatment by aligning the doctor's schemes. Operations Research for Health Care, 7:111-121, 2015.

[48] G. Bitran and R. Caldentey. An overview of pricing models for revenue management. Manufacturing \& Service Operations Management, 5(3):203-229, 2003.

[49] E.L. Blair and C.E. Lawrence. A queueing network approach to health care planning with an application to burn care in New York State. Socio-Economic Planning Sciences, 15(5):207-216, 1981.

[50] J.T. Blake and J. Donald. Mount Sinai Hospital uses integer programming to allocate operating room time. Interfaces, 32(2):63-73, 2002.

[51] H.J. Böckenhauer, D. Komm, R. Královič, and P. Rossmanith. The online knapsack problem: advice and randomization. Theoretical Computer Science, 527: 61-72, 2014.

[52] A.V. den Boer. Dynamic pricing and learning: historical origins, current research, and new directions. Surveys in Operations Research and Management Science, 20 (1):1-18, 2015.

[53] A.V. den Boer, G.M. Koole, R.D. van der Mei, and B. Zwart. Capacity management for a diagnostic medical facility. Working paper (available at www.math.vu.nl/ koole/publications), VU University, Amsterdam, the Netherlands, 2009.

[54] J.A. Bondy and U.S.R. Murty. Graph theory with applications. Macmillan, London, UK, 1976.

[55] M.A. Bonuccelli and S. Pelagatti. Optimal on demand packet scheduling in singlehop multichannel communication systems. In Proceedings of the IEEE International Parallel \& Distributed Processing Symposium, pages 343-352, Cancún, Mexico, 2000. 
[56] S.C. Borst, R. Núñez Queija, and M.J.G. van Uitert. User-level performance of elastic traffic in a differentiated-services environment. Performance Evaluation, 49(1-4):507-519, 2002.

[57] E.D. Boudreaux and E.L. O'Hea. Patient satisfaction in the emergency department: a review of the literature and implications for practice. The Journal of Emergency Medicine, 26(1):13-26, 2004.

[58] T. Bountourelis, L. Luangkesorn, A. Schaefer, L. Maillart, S.G. Nabors, and G. Clermont. Development and validation of a large scale icu simulation model with blocking. In S. Jain, R.R. Creasey, J. Himmelspach, K. P. White, and M. Fu, editors, Proceedings of the Winter Simulation Conference, pages 11431153, Phoenix, USA, 2011. IEEE.

[59] T. Bountourelis, M.Y. Ulukus, J.P. Kharoufeh, and S.G. Nabors. The modeling, analysis, and management of intensive care units. In B.T. Denton, editor, Handbook of Healthcare Operations Management, volume 184 of International Series in Operations Research $\&$ Management Science, chapter 6, pages 153-182. Springer New York, 2013.

[60] S. Bourdais, P. Galinier, and G. Pesant. HIBISCUS: A constraint programming application to staff scheduling in health care. In F. Rossi, editor, Principles and Practice of Constraint Programming, volume 2833 of Lecture Notes in Computer Science, pages 153-167. 2003.

[61] P.F. van Boven (RIVM). Het treekoverleg: streefnormen wachttijden curatieve sector [in Dutch]. In: Volksgezondheid Toekomst Verkenning, Nationale Atlas Volksgezondheid (available at www.zorgatlas.nl/themas/wachtlijsten/wachtlijsten-ziekenhuiszorg). Bilthoven: RIVM, 2007.

[62] E.A. Boyd and I.C. Bilegan. Revenue management and E-commerce. Management Science, 49(10):1363-1386, 2003.

[63] A. Braaksma, N. Kortbeek, G.F. Post, and F. Nollet. Integral multidisciplinary rehabilitation treatment planning. Operations Research for Health Care, 3(3): 145-159, 2014.

[64] A. Braaksma, J. Deglise-Hawkinson, B.T. Denton, M.P. Van Oyen, R.J. Boucherie, and M.R.K. Mes. Online appointment scheduling with different urgencies and appointment lengths. Working paper (obtained through personal communication), 2015.

[65] S. Brailsford. Overcoming the barriers to implementation of operations research simulation models in healthcare. Clinical and Investigative Medicine, 28(6):312$315,2005$.

[66] S. Brailsford and J. Vissers. OR in healthcare: A European perspective. European Journal of Operational Research, 212(2):223-234, 2011.

[67] J.R. Broyles, J.K. Cochran, and D.C. Montgomery. A statistical markov chain approximation of transient hospital inpatient inventory. European Journal of Operational Research, 207(3):1645-1657, 2010. 
[68] A.M. de Bruin, A.C. van Rossum, M.C.C. Visser, and G.M. Koole. Modeling the emergency cardiac in-patient flow: an application of queuing theory. Health Care Management Science, 10(2):125-137, 2007.

[69] A.M. de Bruin, R. Bekker, L. van Zanten, and G.M. Koole. Dimensioning hospital wards using the erlang loss model. Annals of Operations Research, 178(1):23-43, 2010 .

[70] E.K. Burke, P. Leite-Rocha, and S. Petrovic. An integer linear programming model for the radiotherapy treatment scheduling problem. Working paper (available at arxiv:1103.3391v1), 2011.

[71] D. Byrne and B. Silke. Acute medical units: Review of evidence. European Journal of Internal Medicine, 22(4):344-347, 2011.

[72] A.M. Campbell and M.W.P. Savelsbergh. Decision support for consumer direct grocery initiatives. Transportation Science, 39(3):313-327, 2005.

[73] J. Cao and F. Zimmermann. Queue scheduling and advance reservations with cosy. In Proceedings 18th International Parallel and Distributed Processing Symposium, pages 63-70, Santa Fe, New Mexico, April 2004. IEEE.

[74] P. Cao and J. Tang. Hybrid appointment for a single-physician clinic with noshows and overbooking. In Proceedings of the 26th Chinese Control and Decision Conference, pages 2680-2685, Changsha, China, 2014.

[75] P. Cappanera, F. Visintin, and C. Banditori. Comparing resource balancing criteria in master surgical scheduling: A combined optimisation-simulation approach. International Journal of Production Economics, 158:179-196, 2014.

[76] B. Cardoen, E. Demeulemeester, and J. Beliën. Operating room planning and scheduling: a literature review. European Journal of Operational Research, 201 (3):921-932, 2010.

[77] A.P. Carpenter, L.M. Leemis, A.S. Papir, D.J. Phillips, and G.S. Phillips. Managing magnetic resonance imaging machines: support tools for scheduling and planning. Health Care Management Science, 14(2):158-173, 2011.

[78] M.W. Carter and S. Ketabi. Bed balancing in surgical wards via block scheduling. Journal of Minimally Invasive Surgical Sciences, 2(2):129-137, 2013.

[79] C. Castillo, G.N. Rouskas, and K. Harfoush. On the design of online scheduling algorithms for advance reservations and QoS in grids. In Proceedings of the IEEE International Parallel \& Distributed Processing Symposium, pages 1-10, Long Beach, USA, 2007.

[80] C. Castillo, G.N. Rouskas, and K. Harfoush. Online algorithms for advance resource reservations. Journal of Parallel and Distributed Computing, 71(7):963973, 2011.

[81] E. Castro and S. Petrovic. Combined mathematical programming and heuristics for a radiotherapy pre-treatment scheduling problem. Journal of Scheduling, 15 (3):333-346, 2012. 
[82] T. Cayirli and E.D. Gunes. Outpatient appointment scheduling in presence of seasonal walk-ins. Journal of the Operational Research Society, 65(4):512-531, 2013.

[83] T. Cayirli and E. Veral. Outpatient scheduling in health care: a review of literature. Production and Operations Management, 12(4):519-549, 2003.

[84] T. Cayirli, K.K. Yang, and S.A. Quek. A universal appointment rule in the presence of no-shows and walk-ins. Production and Operations Management, 21 (4):682-697, 2012.

[85] S. Ceschia and A. Schaerf. Dynamic patient admission scheduling with operating room constraints, flexible horizons, and patient delays. To appear in Journal of Scheduling. doi: 10.1007/s10951-014-0407-8.

[86] S. Ceschia and A. Schaerf. Local search and lower bounds for the patient admission scheduling problem. Computers \& Operations Research, 38(10):1452-1463, 2011.

[87] S. Ceschia and A. Schaerf. Modeling and solving the dynamic patient admission scheduling problem under uncertainty. Artificial Intelligence in Medicine, 56(3): 199-205, 2012.

[88] R. Chakka, D. Papp, and T. Le-Nhat. A new queueing model for a physician office accepting scheduled patients and patients without appointments. In T. Van Do, H.A.L. Thi, and N.T. Nguyen, editors, Advanced Computational Methods for Knowledge Engineering, volume 282 of Advances in Intelligent Systems and Computing, pages 107-118. Springer International Publishing, Cham, Switzerland, 2014 .

[89] S. Chakraborty, K. Muthuraman, and M. Lawley. Sequential clinical scheduling with patient no-show: the impact of pre-defined slot structures. Socio-Economic Planning Sciences, 47(3):205-219, 2013.

[90] C.W. Chan, V.F. Farias, N. Bambos, and G.J. Escobar. Optimizing intensive care unit discharge decisions with patient readmissions. Operations Research, 60 (6):1323-1341, 2012.

[91] G.J. Chang. On-line job shop scheduling with transfer time in supply chain. In Proceedings of the IEEE International Conference on Automation and Logistics, pages 284-289, Qingdao, China, September 2008.

[92] N. Charbonneau and V.M. Vokkarane. A survey of advance reservation routing and wavelength assignment in wavelength-routed WDM networks. IEEE Communications Surveys \& Tutorials, 14(4):1037-1064, 2012.

[93] S.S. Chauhan, V. Gordon, and J.M. Proth. Scheduling in supply chain environment. European Journal of Operational Research, 183(3):961-970, 2007.

[94] F. Chauvet, E. Levner, L.K. Meyzin, and J.M. Proth. On-line scheduling in a surface treatment system. European Journal of Operational Research, 120(2): 382-392, 2000. 
[95] F. Chauvet, J.M. Proth, and Y. Wardi. Scheduling no-wait production with time windows and flexible processing times. IEEE Transactions on Robotics and Automation, 17(1):60-69, 2001.

[96] B. Chen and G.J. Woeginger. A study of on-line scheduling two-stage shops. In D.Z. Du and P.M. Pardalos, editors, Minimax and Applications, volume 4 of Nonconvex Optimization and its Applications, pages 97-107. Kluwer Academic Publishers, Dordrecht, the Netherlands, 1995.

[97] B. Chen, D. Du, J. Han, and J. Wen. On-line scheduling of small open shops. Discrete Applied Mathematics, 110(2-3):133-150, 2001.

[98] C.Y. Chen, H.A. Liu, and J.Y. Song. Integrated projects planning in IS departments: a multi-period multi-project selection and assignment approach with a computerized implementation. European Journal of Operational Research, 229 (3):683-694, 2013.

[99] R.R. Chen and L.W. Robinson. Sequencing and scheduling appointments with potential call-in patients. Production and Operations Management, 23(9):15221538, 2014.

[100] X. Chen, Z. Xu, G. Dósa, X. Han, and H. Jiang. Semi-online hierarchical scheduling problems with buffer or rearrangements. Information Processing Letters, 113 (4):127-131, 2013.

[101] X. Chen, N. Ding, G. Dósa, X. Han, and H. Jiang. Online hierarchical scheduling on two machines with known total size of low-hierarchy jobs. International Journal of Computer Mathematics, 92(5):873-881, 2015.

[102] M.E. Chernew and J.P. Newhouse. Health care spending growth. Handbook of Health Economics, 2:1-43, 2012.

[103] W.C. Chiang, J.C.H. Chen, and X. Xu. An overview of research on revenue management: current issues and future research. International Journal of Revenue Management, 1(1):97-128, 2007.

[104] S. Choi and W.E. Wilhelm. On capacity allocation for operating rooms. Computers $\&$ Operations Research, 44:174-184, 2014.

[105] V.S. Chow, M.L. Puterman, N. Salehirad, W. Huang, and D. Atkins. Reducing surgical ward congestion through improved surgical scheduling and uncapacitated simulation. Production and Operations Management, 20(3):418-430, 2011.

[106] K. Christodoulopoulos and E. Varvarigos. Routing and scheduling in grids. In Proceedings of the International Conference on Transparent Optical Networks, pages 170-174, Athens, Greece, 2008.

[107] K. Christodoulopoulos, K. Vlachos, E. Varvarigos, L. Stampoulidis, and E. Kehayas. Efficient burst reservation protocol: a hybrid signaling protocol for efficient burst-level reservations and quality-of-service differentiation in optical burst switching networks. Journal of Optical Networking, 5(3):147-158, 2006. 
[108] K. Christodoulopoulos, V. Sourlas, I. Mpakolas, and E. Varvarigos. A comparison of centralized and distributed meta-scheduling architectures for computation and communication tasks in grid networks. Computer Communications, 32(7-10): 1172-1184, 2009.

[109] D. Claudio, G.E. Okudan Kremer, W. Bravo-Llerena, and A. Freivalds. A dynamic multi-attribute utility theorybased decision support system for patient prioritization in the emergency department. IIE Transactions on Healthcare Systems Engineering, 4(1):1-15, 2014.

[110] C. Cleophas and J.F. Ehmke. When are deliveries profitable? Business $\&$ Information Systems Engineering, 6(3):153-163, 2014.

[111] J.K. Cochran and A. Bharti. Stochastic bed balancing of an obstetrics hospital. Health Care Management Science, 9(1):31-45, 2006.

[112] E.G. Coffman Jr. and P. Jelenković. Threshold policies for single-resource reservation systems. SIGMETRICS Performance Evaluation Review, 28(4):9-10, 2001.

[113] E.G. Coffman Jr., P. Jelenković, and B. Poonen. Reservation probabilities. Advances in Performance Analysis, 13:129-158, 1999.

[114] E.G. Coffman Jr., L. Flatto, and P. Jelenković. Interval packing: the vacant interval distribution. The Annals of Applied Probability, 10(1):240-257, 2000.

[115] E.G. Coffman Jr., J. Csirik, G. Galambos, S. Martello, and D. Vigo. Bin packing approximation algorithms: survey and classification. In P.M. Pardalos, D.Z. Du, and R.L. Graham, editors, Handbook of Combinatorial Optimization, volume 1, pages 455-531. Springer Science+Business Media, New York, USA, second edition, 2013.

[116] J.W. Cohen. The single server queue, volume 8 of North-Holland series in applied mathematics and mechanics. North-Holland Publishing Co., Amsterdam, second edition, 1982.

[117] A. Condotta and N.V. Shakhlevich. Scheduling patient appointments via multilevel template: a case study in chemotherapy. Operations Research for Health Care, 3(3):129-144, 2014.

[118] D. Conforti, F. Guerriero, and R. Guido. Optimization models for radiotherapy patient scheduling. 4OR, 6(3):263-278, 2008.

[119] D. Conforti, F. Guerriero, and R. Guido. Non-block scheduling with priority for radiotherapy treatments. European Journal of Operational Research, 201(1): 289-296, 2010.

[120] D. Conforti, F. Guerriero, R. Guido, M.M. Cerinic, and M.L. Conforti. An optimal decision making model for supporting week hospital management. Health Care Management Science, 14(1):74-88, 2011.

[121] D. Conforti, F. Guerriero, R. Guido, and M. Veltri. An optimal decision-making approach for the management of radiotherapy patients. OR Spectrum, 33(1): 123-148, 2011. 
[122] M.W. Cooke, J. Higgins, and P. Kidd. Use of emergency observation and assessment wards: a systematic literature review. Emergency Medicine Journal, 20(2): 138-142, 2003.

[123] A. Costa, F. A. Cappadonna, and S. Fichera. A hybrid genetic algorithm for job sequencing and worker allocation in parallel unrelated machines with sequencedependent setup times. The International Journal of Advanced Manufacturing Technology, 69(9):2799-2817, 2013.

[124] A.X. Costa, S.A. Ridley, A.K. Shahani, P.R. Harper, V. De Senna, and M.S. Nielsen. Mathematical modelling and simulation for planning critical care capacity. Anaesthesia, 58(4):320-327, 2003.

[125] S. Creemers. Appointment-driven queueing systems. Phd thesis, KU Leuven, Leuven, Belgium, 2009.

[126] S. Creemers and M. Lambrecht. An advanced queueing model to analyze appointment-driven service systems. Computers \&3 Operations Research, 36(10): 2773-2785, 2009.

[127] S. Creemers and M. Lambrecht. Queueing models for appointment-driven systems. Annals of Operations Research, 178(1):155-172, 2010.

[128] S. Creemers, J. Beliën, and M. Lambrecht. The optimal allocation of server time slots over different classes of patients. European Journal of Operational Research, 219(3):508-521, 2012.

[129] S. Creemers, P. Colen, and M. Lambrecht. Evaluation of appointment scheduling rules: a multi-performance measures approach. Working paper (available at ssrn.com), KU Leuven, Leuven, Belgium, 2012.

[130] M.L.A.G. Cremers, J.A.S. Gromicho, W.K. Klein Haneveld, and M.H. van der Vlerk. A dynamic service mechanic problem for a housing corporation. In Proceedings of the Cologne-Twente Workshop on Graphs and Combinatorial Optimization, pages 78-81, Gargnano, Italy, 2008.

[131] H. Croonen. Big data in de zorg: niet alleen verzamelen [in Dutch]. Medisch Contact, 01/02:23-26, 2015.

[132] S. Daly, D.A. Campbell, and P.A. Cameron. Short-stay units and observation medicine: a systematic review. Medical Journal of Australia, 178(11):559-563, 2003.

[133] G.B. Dantzig. Linear programming and extensions. Princeton university press, Princeton, USA, 1963.

[134] R. Davies. Simulation for planning services for patients with coronary artery disease. European Journal of Operational Research, 72(2):323-332, 1994.

[135] S. De Vuyst, S. Wittevrongel, D. Fiems, and H. Bruneel. Controlling the delay trade-off between packet flows using multiple reserved places. Performance Evaluation, 65(6-7):484-511, 2008. 
[136] S. De Vuyst, H. Bruneel, and D. Fiems. Computationally efficient evaluation of appointment schedules in health care. European Journal of Operational Research, 237(3):1142-1154, 2014.

[137] J. Deglise-Hawkinson, M. Van Oyen, and B. Roessler. An optimization-based approach to capacity planning for clinical research operations. Working paper (obtained through personal communication), University of Michigan, Ann Arbor, USA, 2014.

[138] O.C. Demirag and J.L. Swann. Capacity allocation to sales agents in a decentralized logistics network. Naval Research Logistics, 54(7):796-810, 2007.

[139] B. Denton and D. Gupta. A sequential bounding approach for optimal appointment scheduling. IIE Transactions, 35(11):1003-1016, 2003.

[140] F. Dexter, J. Ledolter, and R.E. Wachtel. Tactical decision making for selective expansion of operating room resources incorporating financial criteria and uncertainty in subspecialties' future workloads. Anesthesia $\&$ Analgesia, 100(5): 1425-1432, 2005.

[141] N.M. van Dijk and N. Kortbeek. Erlang loss bounds for OT-ICU systems. Queueing Systems, 63(1-4):253-280, 2009.

[142] J. Ding, T. Ebenlendr, J. Sgall, and G. Zhang. Online scheduling of equal-length jobs on parallel machines. In L. Arge, M. Hoffmann, and E. Welzl, editors, Algorithms - ESA 2007, volume 4698 of Lecture Notes in Computer Science, pages 427-438. Springer-Verlag, Berlin Heidelberg, Germany, 2007.

[143] N. Ding, Y. Lan, X. Chen, G. Dósa, H. Guo, and X. Han. Online minimum makespan scheduling with a buffer. International Journal of Foundations of Computer Science, 25(5):525-536, 2014.

[144] G. Dobson, H. Lee, and E. Pinker. A model of ICU bumping. Operations Research, 58(6):1564-1576, 2010.

[145] G. Dobson, S. Hasija, and E.J. Pinker. Reserving capacity for urgent patients in primary care. Production and Operations Management, 20(3):456-473, 2011.

[146] G. Dósa and L. Epstein. Online scheduling with a buffer on related machines. Journal of Combinatorial Optimization, 20(2):161-179, 2010.

[147] K.A. Dowsland. Nurse scheduling with tabu search and strategic oscillation. European Journal of Operational Research, 106(2-3):393 - 407, 1998.

[148] K. Dresner and P. Stone. A multiagent approach to autonomous intersection management. Journal of Artificial Intelligence Research, 31:591-656, 2008.

[149] D. Du. Randomized on-line scheduling similar jobs to minimize makespan on two identical processors. Acta Mathematicae Applicatae Sinica, 21(3):485-488, 2005.

[150] H. Du Kim and J.S. Kim. An online scheduling algorithm for grid computing systems. In M. Li, X.H. Sun, Q. Deng, and J. Ni, editors, Grid and Cooperative Computing, volume 3033 of Lecture Notes in Computer Science, pages 34-39. Springer-Verlag, Berlin Heidelberg, Germany, 2004. 
[151] R.O. Duda, P.E. Hart, and D.G. Stork. Pattern classification. John Wiley \& Sons, Inc., Hoboken, USA, second edition, 2001.

[152] I. Duenyas, D. Gupta, and T.L. Olsen. Control of a single-server tandem queueing system with setups. Operations Research, 46(2):218-230, 1998.

[153] M.B. Dumas. Hospital bed utilization: an implemented simulation approach to adjusting and maintaining appropriate levels. Health Services Research, 20(1): 43-61, 1985.

[154] T. Ebenlendr and J. Sgall. A lower bound for scheduling of unit jobs with immediate decision on parallel machines. In E. Bampis and M. Skutella, editors, Approximation and Online Algorithms, volume 5426 of Lecture Notes in Computer Science, pages 43-52. Springer-Verlag, Berlin Heidelberg, Germany, 2009.

[155] P. Edara and D. Teodorović. Model of an advance-booking system for highway trips. Transportation Research Part C, 16(1):36-53, 2008.

[156] J.F. Ehmke and A.M. Campbell. Customer acceptance mechanisms for home deliveries in metropolitan areas. European Journal of Operational Research, 233 (1):193-207, 2014.

[157] E. El-Darzi, C. Vasilakis, T. Chaussalet, and P.H. Millard. A simulation modelling approach to evaluating length of stay, occupancy, emptiness and bed blocking in a hospital geriatric department. Health Care Management Science, 1(2):143-149, 1998.

[158] O. El-Rifai, T. Garaix, V. Augusto, and X. Xie. A stochastic optimization model for shift scheduling in emergency departments. Health Care Management Science, pages $1-14,2014$.

[159] S.G. Elkhuizen, S.F. Das, P.J.M. Bakker, and J.A.M. Hontelez. Using computer simulation to reduce access time for outpatient departments. Quality and Safety in Health Care, 16(5):382-386, 2007.

[160] P.J. Emstad and B. Feng. Traffic models for reservation systems. In A. Jensen and V.B. Iversen, editors, Teletraffic and datatraffic in a period of change. (Proceedings 13th International Teletraffic Congress), pages 647-652, Amsterdam, the Netherlands, 1991. Elsevier Science Publishers B.V.

[161] L. Epstein. Optimal preemptive on-line scheduling on uniform processors with non-decreasing speed ratios. Operations Research Letters, 29(2):93-98, 2001.

[162] L. Epstein and A. Ganot. Optimal on-line algorithms to minimize makespan on two machines with resource augmentation. Theory of Computing Systems, 42(4): 431-449, 2008.

[163] L. Epstein and H. Zebedat-Haider. Preemptive online scheduling with rejection of unit jobs on two uniformly related machines. Journal of Scheduling, 17(1):87-93, 2014. 
[164] A. Erdelyi and H. Topaloglu. Computing protection level policies for dynamic capacity allocation problems by using stochastic approximation methods. IIE Transactions, 41(6):498-510, 2009.

[165] A. Erdelyi and H. Topaloglu. Approximate dynamic programming for dynamic capacity allocation with multiple priority levels. IIE Transactions, 43(2):129-142, 2010 .

[166] S.A. Erdogan and B. Denton. Dynamic appointment scheduling of a stochastic server with uncertain demand. INFORMS Journal on Computing, 25(1):116-132, 2013.

[167] J.T. van Essen, M. van Houdenhoven, and J.L. Hurink. Clustering clinical departments for wards to achieve a prespecified blocking probability. OR Spectrum, 37(1):243-271, 2015.

[168] U. Faigle and W.M. Nawijn. Note on scheduling intervals on-line. Discrete Applied Mathematics, 58(1):13-17, 1995.

[169] D. Falsini, A. Perugia, and M.M. Schiraldi. An operations management approach for radiology services. In Proceedings of the Conference on Sustainable Development: Industrial Practice, Education \& Research, Bari, Italy, 2010.

[170] R. de Feijter, J.J.M. Evers, and G. Lodewijks. Improving travel-time reliability by the use of trip booking. IEEE Transactions on Intelligent Transportation Systems, $5(4): 288-292,2004$.

[171] J. Feldman, N. Liu, H. Topaloglu, and S. Ziya. Appointment scheduling under patient preference and no-show behavior. Operations Research, 62(4):794-811, 2014 .

[172] Q. Feng, W. Li, W. Shang, and Y. Cai. An on-line scheduling problem of parallel machines with common maintenance time. Journal of Systems Science and Complexity, 26(2):201-208, 2013.

[173] R.B. Ferreira, F.C. Coelli, W.C . Pereira, and R.M.V.R. Almeida. Optimizing patient flow in a large hospital surgical centre by means of discrete-event computer simulation models. Journal of Evaluation in Clinical Practice, 14(6):1031-1037, 2008 .

[174] J. Figueira, S. Greco, and M. Ehrgott, editors. Multiple criteria decision analysis: state of the art surveys, volume 78 of International Series in Operations Research Es Management Science. Springer Science+Business Media, New York, USA, 2005.

[175] G.B. Figueiredo, E.C. Xavier, and N.L.S. da Fonseca. An optimal batch scheduling algorithm for OBS networks. In Proceedings of the IEEE Global Telecommunications Conference, Honolulu, USA, 2009.

[176] I. Foster, M. Fidler, A. Roy, V. Sander, and L. Winkler. End-to-end quality of service for high-end applications. Computer Communications, 27(14):1375-1388, 2004 . 
[177] A. Fügener, E.W. Hans, R. Kolisch, N. Kortbeek, and P.T. Vanberkel. Master surgery scheduling with consideration of multiple downstream units. European Journal of Operational Research, 239(1):227-236, 2014.

[178] A. Fügener, J.O. Brunner, and A. Podtschaske. Duty and workstation rostering considering preferences and fairness: a case study at a department of anaesthesiology. International Journal of Production Research, 2015.

[179] S. Gallivan and M. Utley. Modelling admissions booking of elective in-patients into a treatment centre. IMA Journal of Management Mathematics, 16(3):305$315,2005$.

[180] S. Gallivan and M. Utley. A technical note concerning emergency bed demand. Health Care Management Science, 14(3):250-252, 2011.

[181] L. Garg, S. McClean, M. Barton, B. Meenan, and K. Fullerton. Forecasting hospital bed requirements and cost of care using phase type survival trees. In Proceedings of the 5th IEEE International Conference Intelligent Systems, pages 185-190, London, UK, 2010.

[182] G.M. Garrison and J.L. Pecina. Using the M/G/inf queueing model to predict inpatient family medicine service census and resident workload. Health Informatics Journal, 2015.

[183] N. Geng and X. Xie. Optimizing contracted resource capacity with two advance cancelation modes. European Journal of Operational Research, 221(3):501-512, 2012 .

[184] N. Geng, X. Xie, V. Augusto, and Z. Jiang. A Monte Carlo optimization and dynamic programming approach for managing MRI examinations of stroke patients. IEEE Transactions on Automatic Control, 56(11):2515-2529, 2011.

[185] N. Geng, X. Xie, and Z. Jiang. Capacity reservation and cancellation of critical resources. IEEE Transactions on Automation Science and Engineering, 8(3):470$481,2011$.

[186] N. Geng, X. Xie, and Z. Jiang. Implementation strategies of a contract-based MRI examination reservation process for stroke patients. European Journal of Operational Research, 231(2):371-380, 2013.

[187] Y. Gerchak, D. Gupta, and M. Henig. Reservation planning for elective surgery under uncertain demand for emergency surgery. Management Science, 42(3):321334, 1996.

[188] Y. Gocgun and A. Ghate. Lagrangian relaxation and constraint generation for allocation and advanced scheduling. Computers \& Operations Research, 39(10): 2323-2336, 2012.

[189] Y. Gocgun and M.L. Puterman. Dynamic scheduling with due dates and time windows: an application to chemotherapy patient appointment booking. Health Care Management Science, 17(1):60-76, 2014. 
[190] E. Gomes and M.A. Ribeiro Dantas. An advance reservation mechanism to enhance throughput in an opportunistic high performance computing environment. In Proceedings of the 13th IEEE International Symposium on Network Computing 63 Applications, pages 221-228, Cambridge, USA, 2014.

[191] K. Gopalan and T. Chiueh. Multiresource allocation and scheduling for periodic soft real-time applications. In M.G. Kienzle and P.J. Shenoy, editors, Multimedia Computing and Networking (Proceedings of SPIE), volume 4673, pages 34-45, 2002.

[192] F. Gorunescu, S.I. McClean, and P.H. Millard. A queueing model for bedoccupancy management and planning of hospitals. Journal of the Operational Research Society, 53(1):19-24, 2002.

[193] F. Gorunescu, S.I. McClean, and P.H. Millard. Using a queueing model to help plan bed allocation in a department of geriatric medicine. Health Care Management Science, 5(4):307-312, 2002.

[194] M. Gorunescu, F. Gorunescu, and A. Prodan. Continuous-time Markov model for geriatric patients behavior: Optimization of the bed occupancy and computer simulation. Korean Journal of Computational \& Applied Mathematics, 9(1):185$195,2002$.

[195] R.L. Graham, E.L. Lawler, J.K. Lenstra, and A.H.G. Rinnooy Kan. Optimization and approximation in deterministic sequencing and scheduling: a survey. In P.L. Hammer, E.L. Johnson, and B.H. Korte, editors, Discrete Optimization II, volume 5 of Annals of Discrete Mathematics, pages 287 - 326. Elsevier, 1979.

[196] L.V. Green and V. Nguyen. Strategies for cutting hospital beds: the impact on patient service. Health Services Research, 36:421-442, 2001.

[197] L.V. Green and S. Savin. Reducing delays for medical appointments: a queueing approach. Operations Research, 56(6):1526-1538, 2008.

[198] L.V. Green, S. Savin, and B. Wang. Managing patient service in a diagnostic medical facility. Operations Research, 54(1):11-25, 2006.

[199] A.G. Greenberg, R. Srikant, and W. Whitt. Resource sharing for book-ahead and instantaneous-request calls. IEEE/ACM Transactions on Networking, 7(1): 10-22, 1999.

[200] J. Griffin, S. Xia, S. Peng, and P. Keskinocak. Improving patient flow in an obstetric unit. Health Care Management Science, 15(1):1-14, 2012.

[201] J.D. Griffiths, V. Knight, and I. Komenda. Bed management in a critical care unit. IMA Journal of Management Mathematics, 24(2):137-153, 2013.

[202] J.D. Griffiths, J.E. Williams, and R.M. Wood. Modelling activities at a neurological rehabilitation unit. European Journal of Operational Research, 226(2): 301-312, 2013.

[203] D. Gross, J.F. Shortle, J.M. Thompson, and C.M. Harris. QtsPus software, appendix E, pages 489-492. John Wiley and Sons, Inc., fourth edition, 2008. 
[204] F. Gu, C. Xie, M. Peng, Ç. Çavdar, S. Khan, and N. Ghani. Virtual overlay network scheduling. IEEE Communications Letters, 15(8):893-895, 2011.

[205] J. Guadix, L. Onieva, J. Muñuzuri, and P. Cortés. An overview of revenue management in service industries: an application to car parks. The Service Industries Journal, 31(1):91-105, 2011.

[206] H.H. Guerrero. Demand management strategies for assemble-to-order production environments. International Journal of Production Research, 29(1):39-51, 1991.

[207] M.M. Gunal and M. Pidd. Discrete event simulation for performance modelling in health care: a review of the literature. Journal of Simulation, 4(1):42-51, 2010.

[208] A. Gunawan and H.C. Lau. Master physician scheduling problem. Journal of the Operational Research Society, 64(3):410-425, 2013.

[209] E. Günther, O. Maurer, N. Megow, and A. Wiese. A new approach to online scheduling: approximating the optimal competitive ratio. In S. Khanna, editor, Proceedings of the 24th ACM-SIAM Symposium on Discrete Algorithms, pages 118-128, New Orleans, USA, 2013.

[210] M. Guo, M. Wagner, and C. West. Outpatient clinic scheduling: a simulation approach. In Proceedings of the Winter Simulation Conference, pages 1981-1987, Washington, USA, 2004.

[211] D. Gupta. Surgical suites' operations management. Production and Operations Management, 16(6):689-700, 2007.

[212] D. Gupta and B. Denton. Appointment scheduling in health care: challenges and opportunities. IIE Transactions, 40(9):800-819, 2008.

[213] D. Gupta and W.Y. Wang. Patient appointments in ambulatory care. In R. Hall, editor, Handbook of Healthcare System Scheduling, volume 168 of International Series in Operations Research $\&$ Management Science, chapter 4, pages 65-104. Springer Science+Business Media, Dordrecht, the Netherlands, 2012.

[214] S. Hahn-Goldberg, M.W. Carter, J.C. Beck, M. Trudeau, P. Sousa, and K. Beattie. Dynamic optimization of chemotherapy outpatient scheduling with uncertainty. Health Care Management Science, 17(4):379-392, 2014.

[215] B. Hamidzadeh, L.Y. Kit, and D.J. Lilja. Dynamic task scheduling using online optimization. IEEE Transactions on Parallel and Distributed Systems, 11(11): 1151-1163, 2000.

[216] J.D. Hamilton. Time series analysis. Princeton University Press, Princeton, USA, 1994.

[217] E.W. Hans and P.T. Vanberkel. Operating theatre planning and scheduling. In R. Hall, editor, Handbook of Healthcare System Scheduling, volume 168 of International Series in Operations Research $\&$ Management Science, chapter 5, pages 105-130. Springer Science+Business Media, Dordrecht, the Netherlands, 2012. 
[218] J.J. Harms. Video distribution to the home using advance reservation. In Proceedings of the Local Computer Networks Conference, pages 170-178, Minneapolis, USA, 1997.

[219] J.J. Harms and J.W. Wong. Performance modeling of a channel reservation service. Computer Networks and ISDN Systems, 27(11):1487-1497, 1995.

[220] P.R. Harper and M.A. Pitt. On the challenges of healthcare modelling and a proposed project life cycle for successful implementation. Journal of the Operational Research Society, 55(6):657-661, 2004.

[221] P.R. Harper and A.K. Shahani. Modelling for the planning and management of bed capacities in hospitals. Journal of the Operational Research Society, 53(1): $11-18,2002$.

[222] P.R. Harper, V.A. Knight, and A.H. Marshall. Discrete conditional phase-type models utilising classification trees: Application to modelling health service capacities. European Journal of Operational Research, 219(3):522-530, 2012.

[223] R.A. Harris. Hospital bed requirements planning. European Journal of Operational Research, 25(1):121-126, 1986.

[224] G.W. Harrison, A. Shafer, and M. Macky. Modelling variability in hospital bed occupancy. Health Care Management Science, 8(4):325-334, 2005.

[225] J.C. Hartman and T.C. Perry. Approximating the solution of a dynamic, stochastic multiple knapsack problem. Control and Cybernetics, 35(3):535-550, 2006.

[226] E. He, X. Wang, and J. Leigh. A flexible advance reservation model for multidomain WDM optical networks. In Proceedings of the 3rd International Conference on Broadband Communications, Networks and Systems, San José, USA, 2006.

[227] S. He, M. Sim, and M. Zhang. Data-driven patient scheduling in emergency departments: A hybrid robust-stochastic approach. Nus working papers, 11/2015 (available at www.optimization-online.org), Last modified 24/11/2015.

[228] J.E. Helm, M.P. Van Oyen, and T.R. Rohleder. Priority scheduling in a queueing network with an application to itinerary completion at destination medical centers. Working paper (obtained through personal communication), Kelley School of Business, Indiana University, Bloomington, USA, 2012.

[229] J. Herbots, W. Herroelen, and R. Leus. Dynamic order acceptance and capacity planning on a single bottleneck resource. Naval Research Logistics, 54(8):874-889, 2007.

[230] J. Herbots, W. Herroelen, and R. Leus. Single-pass and approximate dynamicprogramming algorithms for order acceptance and capacity planning. Journal of Heuristics, 16(2):189-209, 2010.

[231] J. Hertz, A. Krogh, and R.G. Palmer. Introduction to the theory of neural computation. Addison-Wesley, Redwood City, USA, 1991. 
[232] L.B. Holm, H. Luras, and F.A. Dahl. Improving hospital bed utilisation through simulation and optimisation: with application to a $40 \%$ increase in patient volume in a Norwegian General Hospital. International Journal of Medical Informatics, 82(2):80-89, 2013.

[233] M. Holte and C. Mannino. The implementor/adversary algorithm for the cyclic and robust scheduling problem in health-care. European Journal of Operational Research, 226(3):551-559, 2013.

[234] N.R. Hoot and D. Aronsky. Systematic review of emergency department crowding: causes, effects, and solutions. Annals of Emergency Medicine, 52(2):126-136, 2008 .

[235] N. Hosseini and K.M. Taaffe. Allocating operating room block time using historical caseload variability. Health Care Management Science, 18(4):419-430, 2015.

[236] H. van Houwelingen. Ziekenhuizen leeg door succes van dagbehandeling [in Dutch]. Newspaper item (available at www.ad.nl), 7-10-2015.

[237] J. Huang, B. Carmeli, and A. Mandelbaum. Control of patient flow in emergency departments, or multiclass queues with deadlines and feedback. Operations Research, 63(4):892-908, 2015.

[238] Y.L. Huang. Ancillary service impact on outpatient scheduling. International Journal of Health Care Quality Assurance, 26(8):746-759, 2013.

[239] Z. Huang, P. Xiao, and D. Liu. An overlapped advance reservation strategy for grid resources. Journal of Information \& Computational Science, 9(8):2211-2220, 2012 .

[240] A. Huggins, D. Claudio, and E. Pérez. Improving resource utilization in a cancer clinic: an optimization model. In Y. Guan and H. Liao, editors, Proceedings of the Industrial and Systems Engineering Research Conference, Montréal, Canada, 2014 .

[241] P.J.H. Hulshof, R.J. Boucherie, J.T. van Essen, E.W. Hans, J.L. Hurink, N. Kortbeek, N. Litvak, P.T. Vanberkel, E. van der Veen, B. Veltman, I.M.H. Vliegen, and M.E. Zonderland. ORchestra: an online reference database of OR/MS literature in health care. Health Care Management Science, 14(4):383-384, 2011.

[242] P.J.H. Hulshof, N. Kortbeek, R.J. Boucherie, E.W. Hans, and P.J.M. Bakker. Taxonomic classification of planning decisions in health care: a structured review of the state of the art in OR/MS. Health Systems, 1(2):129-175, 2012.

[243] P.J.H. Hulshof, R.J. Boucherie, E.W. Hans, and J.L. Hurink. Tactical resource allocation and elective patient admission planning in care processes. Health Care Management Science, 16(2):152-166, 2013.

[244] M.W. Isken, T.J. Ward, and S.J. Littig. An open source software project for obstetrical procedure scheduling and occupancy analysis. Health Care Management Science, 14(1):56-73, 2011. 
[245] S. Ivanov and V. Zhechev. Hotel revenue management - a critical literature review. Tourism, 60(2):175-197, 2012.

[246] N. Izady and D. Worthington. Setting staffing requirements for time dependent queueing networks: the case of accident and emergency departments. European Journal of Operational Research, 219(3):531-540, 2012.

[247] Y. Jacquemin, E. Marcon, and P. Pommier. Towards an improved resolution of radiotherapy scheduling. In Proceedings of the IEEE Workshop on Health Care Management, Venice, Italy, 2010.

[248] S.L. Janak, X. Lin, and C.A. Floudas. A new robust optimization approach for scheduling under uncertainty: II. uncertainty with known probability distribution. Computers \& Chemical Engineering, 31(3):171-195, 2007.

[249] Jeroen Bosch Ziekenhuis. Het Jeroen Bosch Ziekenhuis: werken uit het hart [in Dutch]. Information booklet, June 2013.

[250] Jeroen Bosch Ziekenhuis. Facts and figures of 2014. Infographic by the marketing department of the Jeroen Bosch hospital, November 2015.

[251] Ł. Jeż, J. Schwartz, J. Sgall, and J. Békési. Lower bounds for online makespan minimization on a small number of related machines. Journal of Scheduling, 16 (5):539-547, 2013.

[252] H. Jiang, Z. Pang, and S. Savin. Performance-based contracts for outpatient medical services. Manufacturing \& Service Operations Management, 14(4):654669, 2012.

[253] Y. Jiang, A. Zhang, and J. Hu. Optimal online algorithms on two hierarchical machines with resource augmentation. In B. Fu and D.Z. Du, editors, Computing and Combinatorics, volume 6842 of Lecture Notes in Computer Science, pages 638-648. Springer-Verlag, Berlin Heidelberg, Germany, 2011.

[254] S. Johns. Heuristics to schedule service engineers within time windows. Journal of the Operational Research Society, 46(3):339-346, 1995.

[255] P.E. Joustra, J. Wit, V.M.D. Struben, B.J.H. Overbeek, P. Fockens, and S.G. Elkhuizen. Reducing access times for an endoscopy department by an iterative combination of computer simulation and linear programming. Health Care Management Science, 13(1):17-26, 2010.

[256] G.C. Kaandorp and G. Koole. Optimal outpatient appointment scheduling. Health Care Management Science, 10(3):217-229, 2007.

[257] A. Kaheel and H. Alnuweiri. Batch scheduling algorithms for optical burst switching networks. In R. Boutaba, K. Almeroth, R. Puigjaner, S. Shen, and J.P. Black, editors, NETWORKING 2005. Networking Technologies, Services, and Protocols; Performance of Computer and Communication Networks; Mobile and Wireless Communications Systems, volume 3462 of Lecture Notes in Computer Science, pages 90-101. Springer-Verlag, Berlin Heidelberg, Germany, 2005. 
[258] A.M. Kaheel, H. Alnuweiri, and F. Gebali. A new analytical model for computing blocking probability in optical burst switching networks. IEEE Journal on Selected Areas in Communications, 24(12):120-128, 2006.

[259] S. Karhi and D. Shabtay. Online scheduling of two job types on a set of multipurpose machines. International Journal of Production Economics, 150:155-162, 2014.

[260] N.R. Kaushik, S.M. Figueira, and S.A. Chiappari. Flexible time-windows for advance reservation scheduling. In Proceedings of the IEEE International Symposium on Modeling, Analysis, and Simulation of Computer and Telecommunication Systems, pages 218-225, Monterey, USA, 2006.

[261] K. Keepers and G.W. Harrison. Internal flows and frequency of internal overflows in a large teaching hospital. In S. McClean, P. Millard, E. El-Darzi, and C. Nugent, editors, Intelligent Patient Management, volume 189 (Part 3), pages 185-192. Springer-Verlag Berlin Heidelberg, 2009.

[262] B. Kemper, C.A.J. Klaassen, and M. Mandjes. Optimized appointment scheduling. European Journal of Operational Research, 239(1):243-255, 2014.

[263] G.M. Kern and H.H. Guerrero. A conceptual model for demand management in the assemble-to-order environment. Journal of Operations Management, 9(1): 65-84, 1990.

[264] S. Kharraja, P. Albert, and S. Chaabane. Block scheduling: toward a master surgical schedule. In Proceedings of the International Conference on Service Systems ES Service Management, volume 1, pages 429-435, Troyes, France, 2006.

[265] S. Kim and S. Kim. Differentiated waiting time management according to patient class in an emergency care center using an open jackson network integrated with pooling and prioritizing. Annals of Operations Research, 230(1):35-55, 2013.

[266] S.C. Kim and I. Horowitz. Scheduling hospital services: the efficacy of electivesurgery quotas. Omega, 30(5):335-346, 2002.

[267] S.C. Kim, I. Horowitz, K.K. Young, and T.A. Buckley. Analysis of capacity management of the intensive care unit in a hospital. European Journal of Operational Research, 115(1):36-46, 1999 .

[268] S.C. Kim, I. Horowitz, K.K. Young, and T.A. Buckley. Flexible bed allocation and performance in the intensive care unit. Journal of Operations Management, 18(4):427-443, 2000.

[269] K.J. Klassen and T.R. Rohleder. Scheduling outpatient appointments in a dynamic environment. Journal of Operations Management, 14(2):83-101, 1996.

[270] K.J. Klassen and T.R. Rohleder. Outpatient appointment scheduling with urgent clients in a dynamic, multi-period environment. International Journal of Service Industry Management, 15(2):167-186, 2004.

[271] K.J. Klassen and R. Yoogalingam. Strategies for appointment policy design with patient unpunctuality. Decision Sciences, 45(5):881-911, 2014. 
[272] P.M. Koeleman and G.M. Koole. Optimal outpatient appointment scheduling with emergency arrivals and general service times. IIE Transactions on Healthcare Systems Engineering, 2(1):14-30, 2012.

[273] A. Kokangul. A combination of deterministic and stochastic approaches to optimize bed capacity in a hospital unit. Computer Methods and Programs in Biomedicine, 90(1):56-65, 2008.

[274] D.R.J.C. Koks, M.E. Zonderland, and C. Heringhaus. Development of an observational instrument to determine variations in the patient care process and patient flow among emergency physicians and internists at the emergency department. International Journal of Emergency Medicine, 6(1):1, 2013.

[275] P. Kolesar. A Markovian model for hospital admission scheduling. Management Science, 16(6):B·384-B·396, 1970.

[276] R. Kolisch and S. Sickinger. Providing radiology health care services to stochastic demand of different customer classes. OR Spectrum, 30(2):375-395, 2008.

[277] A. Kolker. Interdependency of hospital departments and hospital-wide patient flows. In R. Hall, editor, Patient Flow, volume 206 of International Series in Operations Research \& Management Science, chapter 2, pages 43-63. Springer US, 2013.

[278] D. König and V. Schmidt. Relationships between time/customer stationary characteristics of tandem queues attended by a single server. Journal of the Operations Research Society of Japan, 27(3):191-204, 1984.

[279] K. Konstanteli, D. Kyriazis, T. Varvarigou, T. Cucinotta, and G. Anastasi. Realtime guarantees in flexible advance reservations. In Proceedings of the 33rd Annual IEEE International Computer Software and Applications Conference, volume 2, pages 67-72, Seattle, USA, 2009.

[280] K. Konstanteli, T. Cucinotta, and T. Varvarigou. Optimum allocation of distributed service workflows with probabilistic real-time guarantees. Service Oriented Computing and Applications, 4(4):229-243, 2010.

[281] N. Kortbeek, M.E. Zonderland, A. Braaksma, I.M.H. Vliegen, R.J. Boucherie, N. Litvak, and E.W. Hans. Designing cyclic appointment schedules for outpatient clinics with scheduled and unscheduled patient arrivals. Performance Evaluation, $80: 5-26,2014$.

[282] N. Kortbeek, A. Braaksma, F.H.F. Smeenk, P.J.M. Bakker, and R.J. Boucherie. Integral resource capacity planning for inpatient care services based on bed census predictions by hour. Journal of the Operational Research Society, 66(7):10611076, 2015.

[283] V. Kostami and A.R. Ward. Managing service systems with an offline waiting option and customer abandonment. Manufacturing 8 Service Operations Management, 11(4):644-656, 2008. 
[284] A.A. Kovacs, B.L. Golden, R.F. Hartl, and S.N. Parragh. Vehicle routing problems in which consistency considerations are important: a survey. Networks, 64(3): 192-213, 2014.

[285] A. Kumar and J. Mo. Models for bed occupancy management of a hospital in singapore. In A. Ali, editor, Proceedings of the 2010 International Conference on Industrial Engineering 8 Operations Management, pages 1-6, Dhaka, Bangladesh, 2010 .

[286] S. Kumar. Modeling hospital surgical delivery process design using system simulation: optimizing patient flow and bed capacity as an illustration. Technology and Health Care, 19(1):1-20, 2011.

[287] R.J. Kusters and P.M.A. Groot. Modelling resource availability in general hospitals design and implementation of a decision support model. European Journal of Operational Research, 88(3):428-445, 1996.

[288] P.J.M. van Laarhoven and E.H.L. Aarts. Simulated Annealing: Theory and Applications, volume 37 of Mathematics and Its Applications. Springer Netherlands, 1987.

[289] L.R. LaGanga and S.R. Lawrence. Appointment overbooking in health care clinics to improve patient service and clinic performance. Production and Operations Management, 21(5):874-888, 2012.

[290] P. Landa, M. Sonnessa, E. Tanfani, and A. Testi. A discrete event simulation model to support bed management. In Proceedings of the 2014 International Conference on Simulation and Modeling Methodologies, Technologies and Applications, pages 901-912, 2014.

[291] C. Langguth and H. Schuldt. Optimizing resource allocation for scientific workflows using advance reservations. In M. Gertz and B. Ludäscher, editors, Scientific and Statistical Database Management, volume 6187 of Lecture Notes in Computer Science, pages 434-451. Springer-Verlag, Berlin Heidelberg, Germany, 2010.

[292] S.D. Lapierre, D. Goldsman, R. Cochran, and J. DuBow. Bed allocation techniques based on census data. Socio-Economic Planning Sciences, 33(1):25-38, 1999.

[293] H.C. Lau. On the complexity of manpower shift scheduling. Computers $\&$ Operations Research, 23(1):93-102, 1996.

[294] A.M. Law. Simulation modeling and analysis. McGraw-Hill, New York, fourth edition, 2007.

[295] A.M. Law and W.D. Kelton. Simulation Modeling and Analysis. McGrawHill, third edition, 2000.

[296] K. Lee, J.Y.T. Leung, and M.L. Pinedo. Makespan minimization in online scheduling with machine eligibility. Annals of Operations Research, 204(1):189-222, 2013.

[297] K. Lee, H.C. Hwang, and K. Lim. Semi-online scheduling with GoS eligibility constraints. International Journal of Production Economics, 153:204-214, 2014. 
[298] S. Lee, D. Min, J. Ryu, and Y. Yih. A simulation study of appointment scheduling in outpatient clinics: open access and overbooking. Simulation, 89(12):1459-1473, 2013.

[299] A. Legrain, M.A. Fortin, N. Lahrichi, and L.M. Rousseau. Online stochastic optimization of radiotherapy patient scheduling. Health Care Management Science, 18(2):110-123, 2015.

[300] P. Leite-Rocha. Novel approaches to radiotherapy treatment scheduling. PhD thesis, University of Nottingham, Nottingham, UK, 2011.

[301] W.A.M. van Lent, J.W. Deetman, H.J. Teertstra, S.H. Muller, E.W. Hans, and W.H. van Harten. Reducing the throughput time of the diagnostic track involving CT scanning with computer simulation. European Journal of Radiology, 81(11): 3131-3140, 2012.

[302] W.A.M. van Lent, P.T. VanBerkel, and W.H. van Harten. A review on the relation between simulation and improvement in hospitals. BMC Medical Informatics 86 Decision Making, 12(1):18, 2012.

[303] R. Levi and C. Shi. Revenue management of reusable resources with advanced reservations. Working paper (available at www-personal.umich.edu/ shicong), Massachusetts Institute of Technology, Cambridge, USA, 2011.

[304] B. Li and D. Zhao. Performance impact of advance reservations from the grid on backfill algorithms. In Proceedings of the 6th International Conference on Grid \& Cooperative Computing, pages 456-461, Urumchi, China, 2007.

[305] B. Li, Y. Pei, H. Wu, and B. Shen. Resource availability-aware advance reservation for parallel jobs with deadlines. The Journal of Supercomputing, 68(2):798-819, 2014 .

[306] R. Li, L. Yang, X. He, Q. Chen, and X. Cheng. Semi-online scheduling for jobs with release times. Journal of Combinatorial Optimization, 26(3):448-464, 2013.

[307] S. Li, N. Geng, and X. Xie. A queueing and patient pooling approach for healthcare resource allocation with waiting time targets and re-entrance. Working paper (available at www.emse.fr/ xie), Shanghai Jiao Tong University, Shanghai, China, 2014.

[308] X. Li, P. Beullens, D. Jones, and M. Tamiz. An integrated queuing and multiobjective bed allocation model with application to a hospital in china. Journal of the Operational Research Society, 60:330-338, 2009.

[309] X. Li, R.Y.K. Fung, and H. Sun. An events-driven scheduling algorithm for two-cluster tools with processing time windows. In Proceedings of the IEEE International Conference on Industrial Engineering and Engineering Management, pages 1436-1440, Singapore, 2011.

[310] J. Lian, K. DiStefano, S.D. Shields, C. Heinichen, M. Giampietri, and L. Wang. Clinical appointment process: improvement through schedule defragmentation. IEEE Engineering in Medicine \& Biology Magazine, 29(2):127-134, 2010. 
[311] Y. Liang, K-Q. Liao, J.W. Roberts, and A. Simonian. Queueing models for reserved set up telecommunications services. In M. Bonatti, editor, Teletraffic science for new cost-effective systems, networks and services. (Proceedings 12th International Teletraffic Congress), Amsterdam, the Netherlands, 1989. NorthHolland.

[312] J. Lin, K. Muthuraman, and M. Lawley. Optimal and approximate algorithms for sequential clinical scheduling with no-shows. IIE Transactions on Healthcare Systems Engineering, 1(1):20-36, 2011.

[313] R. Litjens and R.J. Boucherie. Performance analysis of fair channel sharing policies in an integrated cellular voice/data network. Telecommunication Systems, 19 (2):147-186, 2002.

[314] R. Litjens, H. van den Berg, and R.J. Boucherie. Throughputs in processor sharing models for integrated stream and elastic traffic. Performance Evaluation, 65(2): 152-180, 2008.

[315] N. Litvak, M. van Rijsbergen, R.J. Boucherie, and M. van Houdenhoven. Managing the overflow of intensive care patients. European Journal of Operational Research, 185(3):998-1010, 2008.

[316] K. Liu, S.H. Son, V.C.S. Lee, and K. Kapitanova. A token-based admission control and request scheduling in lane reservation systems. In Proceedings of the 14th IEEE International Conference on Intelligent Transportation Systems, pages 1489-1494, Washington, USA, 2011.

[317] K. Liu, E. Chan, V. Lee, K. Kapitanova, and S.H. Son. Design and evaluation of token-based reservation for a roadway system. Transportation Research Part $C$, 26:184-202, 2013.

[318] L. Liu and X. Liu. Dynamic and static job allocation for multi-server systems. IIE Transactions, 30(9):845-854, 1998.

[319] M. Liu, F. Zheng, C. Chu, and Y. Xu. Optimal algorithms for online scheduling on parallel machines to minimize the makespan with a periodic availability constraint. Theoretical Computer Science, 412(39):5225-5231, 2011.

[320] N. Liu. Optimal choice for appointment scheduling window under patient no-show behavior. Production and Operations Management, 25(1):128-142, 2016.

[321] N. Liu and S. Ziya. Panel size and overbooking decisions for appointment-based services under patient no-shows. Production and Operations Management, 23(12): 2209-2223, 2014.

[322] N. Liu, S. Ziya, and V.G. Kulkarni. Dynamic scheduling of outpatient appointments under patient no-shows and cancellations. Manufacturing $\& 3$ Service Operations Management, 12(2):347-364, 2010.

[323] X. Lu and Z. Liu. Semi-online scheduling problems on two uniform machines under a grade of service provision. Theoretical Computer Science, 489-490:58-66, 2013. 
[324] Y. Lu and A. Radovanović. Asymptotic blocking probabilities in loss networks with subexponential demands. Journal of Applied Probability, 44:1088-1102, 2007.

[325] J. Luo, V.G. Kulkarni, and S. Ziya. Appointment scheduling under patient noshows and service interruptions. Manufacturing 85 Service Operations Management, 14(4):670-684, 2012.

[326] J. Luo, Z. Wu, J. Cao, and T. Tian. Dynamic multi-resource advance reservation in grid environment. The Journal of Supercomputing, 60(3):420-436, 2012.

[327] J. Luo, V.G. Kulkarni, and S. Ziya. A tandem queueing model for an appointmentbased service system. Queueing Systems, 79(1):53-85, 2015.

[328] T. Luo and Y. Xu. Semi-online scheduling on two machines with GoS levels and partial information of processing time. The Scientific World Journal, 2014:Article ID 576234, 2014.

[329] T. Luo, Y. Xu, L. Luo, and C. He. Semi-online scheduling with two GoS levels and unit processing time. Theoretical Computer Science, 521:62-72, 2014.

[330] Y. Luzon, A. Mandelbaum, and M. Penn. Scheduling appointments via fluids control. In Proceedings of the International Conference on Model-Based Systems Engineering, pages 29-35, Haifa, Israel, 2009.

[331] G. Ma and E. Demeulemeester. A multilevel integrative approach to hospital case mix and capacity planning. Computers \&S Operations Research, 40(9):2198-2207, 2013.

[332] C.M. Macal and M.J. North. Tutorial on agent-based modelling and simulation. Journal of Simulation, 4:151-162, 2010.

[333] M. Mackay. Practical experience with bed occupancy management and planning systems: an australian view. Health Care Management Science, 4(1):47-56, 2001.

[334] M. Mackay and M. Lee. Choice of models for the analysis and forecasting of hospital beds. Health Care Management Science, 8(3):221-230, 2005.

[335] O.B.G. Madsen, K. Tosti, and J. Vælds. A heuristic method for dispatching repair men. Annals of Operations Research, 61(1):213-226, 1995.

[336] M. Mahdavi, T. Malmstrom, J. van de Klundert, S. Elkhuizen, and J. Vissers. Generic operational models in health service operations management: A systematic review. Socio-Economic Planning Sciences, 47(4):271-280, 2013.

[337] R.J. Maillardet and P.G. Taylor. Queues with advanced reservations: an infiniteserver proxy for the bookings diary. Advances in Applied Probability, 48(1):13-31, 2016.

[338] M. Mainegra Hing, A. van Harten, and P.C. Schuur. Reinforcement learning versus heuristics for order acceptance on a single resource. Journal of Heuristics, 13(2):167-187, 2007. 
[339] F. Mallor and C. Azcarate. Combining optimization with simulation to obtain credible models for intensive care units. Annals of Operations Research, 221(1): 255-271, 2014.

[340] F. Mallor, C. Azcarate, and J. Barado. Control problems and management policies in health systems: application to intensive care units. Flexible Services and Manufacturing Journal, 28(1):62-89, 2016.

[341] A. Mamat, Y. Lu, J. Deogun, and S. Goddard. Real-time divisible load scheduling with advance reservation. In Proceedings of the Euromicro Conference on RealTime Systems, pages 37-46, Prague, Czech Republic, 2008.

[342] C. Mannino, E.J. Nilssen, and T.E. Nordlander. A pattern based, robust approach to cyclic master surgery scheduling. Journal of Scheduling, 15(5):553-563, 2012.

[343] E. Marcon, S. Kharraja, N. Smolski, B. Luquet, and J.P. Viale. Determining the number of beds in the postanesthesia care unit: a computer simulation flow approach. Anesthesia \& Analgesia, 96(5):1415-1423, 2003.

[344] C.C. Marinagi, C.D. Spyropoulos, C. Papatheodorou, and S. Kokkotos. Continual planning and scheduling for managing patient tests in hospital laboratories. Artificial Intelligence in Medicine, 20(2):139-154, 2000.

[345] Y.N. Marmor, T.R. Rohleder, D.J. Cook, T.R. Huschka, and J.E. Thompson. Recovery bed planning in cardiovascular surgery: a simulation case study. Health Care Management Science, 16(4):314-327, 2013.

[346] I.H.J. Masselink, T.L.C. van der Mijden, N. Litvak, and P.T. Vanberkel. Preparation of chemotherapy drugs: planning policy for reduced waiting times. Omega, 40(2):181 - 187, 2012.

[347] W.A. Massey and W. Whitt. An analysis of the modified offered-load approximation for the nonstationary Erlang loss model. The Annals of Applied Probability, 4(4):1145-1160, 1994.

[348] B.J. Masterson, T.G. Mihara, G. Miller, S.C. Randolph, M.E. Forkner, and A.L. Crouter. Using models and data to support optimization of the military health system: A case study in an intensive care unit. Health Care Management Science, $7(3): 217-224,2004$.

[349] M. Matsumoto and T. Nishimura. Mersenne twister: A 623-dimensionally equidistributed uniform pseudo-random number generator. In ACM Transactions on Modeling and Computer Simulation, volume 8 (1), pages 3-30, New York, USA, January 1998. ACM.

[350] M.E. Matta and S.S. Patterson. Evaluating multiple performance measures across several dimensions at a multi-facility outpatient center. Health Care Management Science, 10(2):173-194, 2007. ISSN 1386-9620.

[351] A. Mazier, X. Xie, and M. Sarazin. Scheduling inpatient admission under high demand of emergency patients. In Proceedings of the IEEE International Conference on Automation Science and Engineering, pages 792-797, Toronto, Canada, 2010 . 
[352] J.O. McClain. A model for regional obstetric bed planning. Health Services Research, 13(4):378-394, 1978.

[353] J.I. McGill and G.J. Van Ryzin. Revenue management: research overview and prospects. Transportation Science, 33(2):233-256, 1999.

[354] A.S. McGough, A. Afzal, J. Darlington, N. Furmento, A. Mayer, and L. Young. Making the grid predictable through reservations and performance modelling. The Computer Journal, 48(3):358-368, 2005.

[355] F. McLeod and T. Cherrett. Loading bay booking and control for urban freight. International Journal of Logistics: Research and Applications, 14(6):385-397, 2011.

[356] M.L. McManus, M.C. Long, A. Cooper, and E. Litvak. Queuing theory accurately models the need for critical care resources. Anesthesiology, 100(5):1271-1276, 2004 .

[357] M. Mes, M. van der Heijden, and P. Schuur. Look-ahead strategies for dynamic pickup and delivery problems. OR Spectrum, 32(2):395-421, 2010.

[358] R. Min and M. Maheswaran. Scheduling co-reservations with priorities in grid computing systems. In Proceedings of the IEEE/ACM International Symposium on Cluster Computing and the Grid, Berlin, Germany, 2002.

[359] X. Min, Y. Wang, J. Liu, and M. Jiang. Semi-online scheduling on two identical machines with rejection. Journal of Combinatorial Optimization, 26(3):472-479, 2013.

[360] M. Miyazawa and G. Yamazaki. The basic equations for a supplemented gsmp and its applications to queues. Journal of Applied Probability, 25:565-578, 1988.

[361] H.R. Moaddeli, G. Dastghaibyfard, and M.R. Moosavi. Flexible advance reservation impact on backfilling scheduling strategies. In Proceedings of the 7th International Conference on Grid and Cooperative Computing, pages 151-159, Shenzhen, China, 2008.

[362] S.V. Mondschein and G.Y. Weintraub. Appointment policies in service operations: a critical analysis of the economic framework. Production and Operations Management, 12(2):266-286, 2003.

[363] M. Müller-Bungart. Revenue management with flexible products: models and methods for the broadcasting industry, volume 596 of Lecture Notes in Economics and Mathematical Systems. Springer-Verlag, Berlin Heidelberg, Germany, 2007.

[364] N. Mustafee, T. Lyons, P. Rees, L. Davies, M. Ramsey, and M.D. Williams. Planning of bed capacities in specialized and integrated care units: incorporating bed blockers in a simulation of surgical throughput. In C. Laroque, J. Himmelspach, R. Pasupathy, O. Rose, and A.M. Uhrmacher, editors, Proceedings of the Winter Simulation Conference, pages 1-12. IEEE, 2012.

[365] K. Muthuraman and M. Lawley. A stochastic overbooking model for outpatient clinical scheduling with no-shows. IIE Transactions, 40(9):820-837, 2008. 
[366] R.B. Myerson. Game theory: analysis of conflict. Harvard university press, Cambridge, MA, 1991.

[367] J. Nair, A. Wierman, and B. Zwart. Tail-robust scheduling via limited processor sharing. Performance Evaluation, 67(11):978-995, 2010.

[368] Nationaal Borstkanker Overleg Nederland. Nabon-nota handboek organisatie mammazorg [in Dutch], 2008.

[369] National Institute for Public Health and the Environment (RIVM). Screening for breast cancer. Information booklet, 2012.

[370] Nederlandse Vereniging van Ziekenhuizen. Zorg in de toekomst: standpunten [in Dutch]. Booklet (available at www.nvz-ziekenhuizen.nl), Utrecht, 2013.

[371] Netherlands Cancer Registry (IKNL). Most prevalent localizations of cancer in 2013. Online database (available at www.cijfersoverkanker.nl), accessed 10-092014.

[372] M.E.J. Newman. Networks: an introduction. Oxford University Press, Oxford, UK, 2010.

[373] J.M. Nguyen, P. Six, R. Parisot, D. Antonioli, F. Nicolas, and P. Lombrail. A universal method for determining intensive care unit bed requirements. Intensive Care Medicine, 29(5):849-852, 2003.

[374] T.B.T. Nguyen, A.I. Sivakumar, and S.C. Graves. A network flow approach for tactical resource planning in outpatient clinics. Health Care Management Science, 18(2):124-136, 2015.

[375] M. Nowak, M. Hewitt, and N. Nataraj. Planning strategies for home health care delivery. Iie transactions on healthcare systems engineering (available at ecommons.luc.edu), 2013.

[376] L.G.N. Nunes, S.V. de Carvalho, and R. de Cássia Meneses Rodrigues. Markov decision process applied to the control of hospital elective admissions. Artificial Intelligence in Medicine, 47(2):159-171, 2009.

[377] J.P. Oddoye, M.A. Yaghoobi, M. Tamiz, D.F. Jones, and P. Schmidt. A multiobjective model to determine efficient resource levels in a medical assessment unit. Journal of the Operational Research Society, 58(12):1563-1573, 2007.

[378] J.P. Oddoye, D.F. Jones, M. Tamiz, and P. Schmidt. Combining simulation and goal programming for healthcare planning in a medical assessment unit. European Journal of Operational Research, 193(1):250-261, 2009.

[379] S.N. Ogulata, M.O. Cetik, E. Koyuncu, and M. Koyuncu. A simulation approach for scheduling patients in the department of radiation oncology. Journal of Medical Systems, 33(3):233-239, 2009.

[380] H.J. Oh, A. Muriel, H. Balasubramanian, K. Atkinson, and T. Ptaszkiewicz. Guidelines for scheduling in primary care under different patient types and stochastic nurse and provider service times. IIE Transactions on Healthcare Systems Engineering, 3(4):263-279, 2013. 
[381] J.M. van Oostrum, M. van Houdenhoven, J.L. Hurink, E.W. Hans, G. Wullink, and G. Kazemier. A master surgical scheduling approach for cyclic scheduling in operating room departments. OR Spectrum, 30(2):355-374, 2008.

[382] T. Orawiwattanakul, H. Otsuki, E. Kawai, and S. Shimojo. Dynamic time scheduling in advance bandwidth reservation. In Proceedings of the 26th International Conference on Advanced Information Networking and Applications, pages 885890, Fukuoka, Japan, 2012.

[383] A.C. Orgerie, L. Lefèvre, and I. Guérin-Lassous. Energy-efficient bandwidth reservation for bulk data transfers in dedicated wired networks. The Journal of Supercomputing, 62(3):1139-1166, 2012.

[384] K. Pak and N. Piersma. Overview of OR techniques for airline revenue management. Statistica Neerlandica, 56(4):479-495, 2002.

[385] J. Patrick and A. Aubin. Models and methods for improving patient access. In B.T. Denton, editor, Handbook of Healthcare Operations Management, volume 184 of International Series in Operations Research $\&$ Management Science, chapter 15, pages 403-420. Springer Science+Business Media, Dordrecht, the Netherlands, 2013.

[386] J. Patrick and M.L. Puterman. Improving resource utilization for diagnostic services through flexible inpatient scheduling: a method for improving resource utilization. Journal of the Operational Research Society, 58(2):235-245, 2007.

[387] J. Patrick, M.L. Puterman, and M. Queyranne. Dynamic multipriority patient scheduling for a diagnostic resource. Operations Research, 56(6):1507-1525, 2008.

[388] S.A. Paul, M.C. Reddy, and C.J. DeFlitch. A systematic review of simulation studies investigating emergency department overcrowding. Simulation, 86(8-9): $559-571,2010$.

[389] C. Pehlivan, V. Augusto, X. Xiaolan, and C. Crenn-Hebert. Multi-period capacity planning for maternity facilities in a perinatal network: A queuing and optimization approach. In Proceedings of the 2012 IEEE International Conference on Automation Science and Engineering, pages 137-142, 2012.

[390] Y. Peng, X. Qu, and J. Shi. A hybrid simulation and genetic algorithm approach to determine the optimal scheduling templates for open access clinics admitting walk-in patients. Computers \& Industrial Engineering, 72:282-296, 2014.

[391] E. Pérez, L. Ntaimo, C. Bailey, and P. McCormack. Modeling and simulation of nuclear medicine patient service management in DEVS. Simulation, 86(8-9): 481-501, 2010.

[392] E. Pérez, L. Ntaimo, W.E. Wilhelm, C. Bailey, and P. McCormack. Patient and resource scheduling of multi-step medical procedures in nuclear medicine. IIE Transactions on Healthcare Systems Engineering, 1(3):168-184, 2011.

[393] E. Pérez, L. Ntaimo, C.O. Malavé, C. Bailey, and P. McCormack. Stochastic online appointment scheduling of multi-step sequential procedures in nuclear medicine. Health Care Management Science, 16(4):281-299, 2013. 
[394] T.C. Perry and J.C. Hartman. An approximate dynamic programming approach to solving a dynamic, stochastic multiple knapsack problem. International Transactions in Operational Research, 16(3):347-359, 2009.

[395] H. Peters. Game theory: a multi-leveled approach. Springer-Verlag, Berlin Heidelberg, Germany, 2008.

[396] D. Petrovic, E. Castro, S. Petrovic, and T. Kapamara. Radiotherapy scheduling. In A.S. Uyar, E. Ozcan, and N. Urquhart, editors, Automated Scheduling and Planning, volume 505 of Studies in Computational Intelligence, pages 155-189. Springer-Verlag, Berlin Heidelberg, Germany, 2013.

[397] S. Petrovic and P. Leite-Rocha. Constructive approaches to radiotherapy scheduling. In Proceedings of the World Congress on Engineering and Computer Science, pages 722-727, San Francisco, USA, 2008.

[398] S. Petrovic, W. Leung, X. Song, and S. Sundar. Algorithms for radiotherapy treatment booking. In Proceedings of the Workshop of the UK Planning and Scheduling Special Interest Group, Nottingham, UK, 2006.

[399] V. Pillac, M. Gendreau, C. Guéret, and A.L. Medaglia. A review of dynamic vehicle routing problems. European Journal of Operational Research, 225(1):111, 2013.

[400] M. Pinedo, C. Zacharias, and N. Zhu. Scheduling in the service industries: an overview. Journal of Systems Science and Systems Engineering, 24(1):1-48, 2015.

[401] M.L. Pinedo. Scheduling: theory, algorithms and systems. Springer Science+Business Media, Dordrecht, the Netherlands, fourth edition, 2012.

[402] W.B. Powell. Approximate dynamic programming: solving the curses of dimensionality. Wiley Series in Probability and Statistics. John Wiley \& Sons, Inc., Hoboken, USA, second edition, 2011.

[403] L. Prechelt. An empirical comparison of seven programming languages. Computer, 33(10):23-29, 2000.

[404] K. Pruhs, J. Sgall, and E. Torng. Online scheduling. In J.Y.T. Leung, editor, Handbook of scheduling: algorithms, models, and performance analysis, Chapman $\&$ Hall/CRC Computer and Information Science Series, chapter 15, pages 15·115·41. CRC Press LLC, Boca Raton, FL, 2004.

[405] M.L. Puterman. Markov decision processes: discrete stochastic dynamic programming. Wiley Series in Probability and Statistics. John Wiley \& Sons, Inc., Hoboken, USA, 2005.

[406] C. Qiao and M. Yoo. Optical burst switching (OBS) - a new paradigm for an optical internet. Journal of High Speed Networks, 8(1):69-84, 1999.

[407] X. Qu, R.L. Rardin, J.A.S. Williams, and D.R. Willis. Matching daily healthcare provider capacity to demand in advanced access scheduling systems. European Journal of Operational Research, 183(2):812-826, 2007. 
[408] X. Qu, R.L. Rardin, and J.A.S. Williams. A mean-variance model to optimize the fixed versus open appointment percentages in open access scheduling systems. Decision Support Systems, 53(3):554-564, 2012.

[409] X. Qu, Y. Peng, N. Kong, and J. Shi. A two-phase approach to scheduling multicategory outpatient appointments - a case study of a women's clinic. Health Care Management Science, 16(3):197-216, 2013.

[410] R. Núñez Queija. Processor-sharing models for integrated-services networks. PhD thesis, Eindhoven University of Technology, 2000.

[411] H. Rahman, R. Sarker, and D. Essam. Permutation flow shop scheduling with dynamic job order arrival. In Proceedings of the IEEE Conference on Cybernetics and Intelligent Systems, pages 30-35, Manila and Pico de Loro, Philippines, 2013.

[412] K. Rajah, S. Ranka, and Y. Xia. Advance reservations and scheduling for bulk transfers in research networks. IEEE Transactions on Parallel and Distributed Systems, 20(11):1682-1697, 2009.

[413] M. Ramakrishnan, D. Sier, and P.G. Taylor. A two-time-scale model for hospital patient flow. IMA Journal of Management Mathematics, 16(3):197-215, 2005.

[414] A. Ratcliffe, W. Gilland, and A. Marucheck. Revenue management for outpatient appointments: joint capacity control and overbooking with class-dependent noshows. Flexible Services and Manufacturing Journal, 24(4):516-548, 2012.

[415] A. Rhodes, P. Ferdinande, H. Flaatten, B. Guidet, P.G. Metnitz, and R.P. Moreno. The variability of critical care bed numbers in Europe. Intensive Care Medicine, 38(10):1647-1653, 2012.

[416] J.C. Ridge, S.K. Jones, M.S. Nielsen, and A.K. Shahani. Capacity planning for intensive care units. European Journal of Operational Research, 105(2):346-355, 1998.

[417] A. Riise and E.K. Burke. Local search for the surgery admission planning problem. Journal of Heuristics, 17(4):389-414, 2011.

[418] J. Roberts and K. Liao. Traffic models for telecommunication services with advance capacity reservation. Computer Networks and ISDN systems, 10(3-4):221$229,1985$.

[419] M. Roelofs and J. Bisschop. AIMMS: the user's guide, chapter 20, pages 235-244. Paragon Decision Technology, 2012.

[420] T.R. Rohleder and K.J. Klassen. Rolling horizon appointment scheduling: a simulation study. Health Care Management Science, 5(3):201-209, 2002.

[421] H.L. Romero, N.P. Dellaert, S. van der Geer, M. Frunt, M.H. Jansen-Vullers, and G.A.M. Krekels. Admission and capacity planning for the implementation of onestop-shop in skin cancer treatment using simulation-based optimization. Health Care Management Science, 16(1):75-86, 2013. 
[422] M. Roser. Life expectancy. Online database, accessed December 2015 (available at ourworldindata.org).

[423] S.M. Ross. Introduction to probability models. Academic Press, San Diego, CA, nineth edition, 2007.

[424] F. Rossi, P. van Beek, and T. Walsh, editors. Handbook of constraint programming. Foundations of Artificial Intelligence. Elsevier, Amsterdam, the Netherlands, first edition, 2006.

[425] A. Sadki, X. Xie, and F. Chauvin. Patients assignment for an oncology outpatient unit. In Proceedings of the IEEE International Conference on Automation Science and Engineering, pages 891-896, Toronto, Canada, 2010.

[426] A. Sadki, X. Xie, and F. Chauvin. Planning oncologists of ambulatory care units. Decision Support Systems, 55(2):640-649, 2013.

[427] J.R.C. van Sambeek, F.A. Cornelissen, P.J.M. Bakker, and J.J. Krabbendam. Models as instruments for optimizing hospital processes: a systematic review. International Journal of Health Care Quality Assurance, 23(4):356-377, 2010.

[428] J.R.C. van Sambeek, P.E. Joustra, S.F. Das, P.J. Bakker, and M. Maas. Reducing MRI access times by tackling the appointment-scheduling strategy. BMJ Quality ES Safety, 20(12):1075-1080, 2011.

[429] M. Samorani and L.R. LaGanga. Outpatient appointment scheduling given individual day-dependent no-show predictions. European Journal of Operational Research, 240(1):245-257, 2015.

[430] P. Santibáñez, M. Begen, and D. Atkins. Surgical block scheduling in a system of hospitals: an application to resource and wait list management in a British Columbia health authority. Health Care Management Science, 10(3):269-282, 2007.

[431] P. Santibáñez, R. Aristizabal, M.L. Puterman, V.S. Chow, W. Huang, C. Kollmannsberger, T. Nordin, N. Runzer, and S. Tyldesley. Operations research methods improve chemotherapy patient appointment scheduling. The Joint Commission Journal on Quality and Patient Safety, 38(12):541-553, 2012.

[432] A. Sauré and M.L. Puterman. The appointment scheduling game. INFORMS Transactions on Education, 14(2):73-85, 2014.

[433] A. Sauré, J. Patrick, S. Tyldesley, and M.L. Puterman. Dynamic multiappointment patient scheduling for radiation therapy. European Journal of Operational Research, 223(2):573-584, 2012.

[434] O. Schelén and S. Pink. Resource sharing in advance reservation agents. Journal of High Speed Networks, 7(3-4):213-228, 1998.

[435] R. Schmidt, S. Geisler, and C. Spreckelsen. Decision support for hospital bed management using adaptable individual length of stay estimations and shared resources. BMC Medical Informatics \& Decision Making, 13(1):1-19, 2013. 
[436] M. Scholtens. Visiting the CT-scan; appointment system or walk in? Patient preferences and possible arrival pattern. Master's thesis, University of Twente, 2009 .

[437] L. Schrage. Letter to the editor - a proof of the optimality of the shortest remaining processing time discipline. Operations Research, 16(3):687-690, 1968.

[438] C.R. Schultz. Economic service quotas. Journal of Service Research, 5(2):154-163, 2002 .

[439] I. Scott, L. Vaughan, and D. Bell. Effectiveness of acute medical units in hospitals: a systematic review. International Journal for Quality in Health Care, 21(6):397407,2009 .

[440] K. Seklou, A. Sideri, P. Kokkinos, and E. Varvarigos. New assembly techniques and fast reservation protocols for optical burst switched networks based on traffic prediction. Optical Switching and Networking, 10(2):132-148, 2013.

[441] Suvrajeet Sen. Algorithms for stochastic mixed-integer programming models. In G.L. Nemhauser K. Aardal and R. Weismantel, editors, Discrete Optimization, volume 12 of Handbooks in Operations Research and Management Science, pages 515 - 558. Elsevier, 2005.

[442] L.I. Sennott, M.P. Van Oyen, and S.M.R. Iravani. Optimal dynamic assignment of a flexible worker on an open production line with specialists. European Journal of Operational Research, 170(2):541 - 566, 2006.

[443] J. Sgall. On-line scheduling. In A. Fiat and G.J. Woeginger, editors, Online Algorithms, volume 1442 of Lecture Notes in Computer Science, chapter 9, pages 196-231. Springer-Verlag, Berlin Heidelberg, Germany, 1998.

[444] D. Shabtay and S. Karhi. An asymptotically optimal online algorithm to minimize the total completion time on two multipurpose machines with unit processing times. Discrete Optimization, 9(4):241-248, 2012.

[445] A.K. Shahani, S.A. Ridley, and M.S. Nielsen. Modelling patient flows as an aid to decision making for critical care capacities and organisation. Anaesthesia, 63 (10):1074-1080, 2008.

[446] A. Shaik, M, S.L. Janak, and C.A. Floudas. Continuous-time models for shortterm scheduling of multipurpose batch plants: A comparative study. Industrial E Engineering Chemistry Research, 45(18):6190-6209, 2006.

[447] O. Shcherbina and E. Shembeleva. Modeling recreational systems using optimization techniques and information technologies. Annals of Operations Research, 221 (1):309-329, 2014.

[448] C.C. Shen and W.H. Tsai. A graph matching approach to optimal task assignment in distributed computing systems using a minimax criterion. IEEE Transactions on Computers, C-34(3):197-203, 1985.

[449] A. Shmueli, C.L. Sprung, and E.H. Kaplan. Optimizing admissions to an intensive care unit. Health Care Management Science, 6(3):131-136, 2003. 
[450] W. Shonick and J.R. Jackson. An improved stochastic model for occupancyrelated random variables in general-acute hospitals. Operations Research, 21(4): 952-965, 1973.

[451] S. Sickinger and R. Kolisch. The performance of a generalized Bailey-Welch rule for outpatient appointment scheduling under inpatient and emergency demand. Health Care Management Science, 12(4):408-419, 2009.

[452] E. Simhon and D. Starobinski. Game-theoretic analysis of advance reservation services. In Proceedings of the 48th Annual Conference on Information Sciences and Systems, Princeton, USA, 2014.

[453] A.A. Sissouras and B. Moores. The optimum number of beds in a coronary care unit. Omega, 4(1):59-65, 1976.

[454] S.A. Slotnick. Order acceptance and scheduling: a taxonomy and review. European Journal of Operational Research, 212(1):1-11, 2011.

[455] W. Smith, I. Foster, and V. Taylor. Scheduling with advanced reservations. In Proceedings of the 14th International Parallel 85 Distributed Processing Symposium, pages 127-132, Cancún, Mexico, 2000. IEEE.

[456] Q. Snell, M. Clement, D. Jackson, and C. Gregory. The performance impact of advance reservation meta-scheduling. In D.G. Feitelson and L. Rudolph, editors, Job Scheduling Strategies for Parallel Processing, volume 1911 of Lecture Notes in Computer Science, pages 137-153. Springer-Verlag, Berlin Heidelberg, Germany, 2000 .

[457] Q. Song, Z. Li, and I. Habib. Performance analysis of connection oriented services with book-ahead reservations in internet protocol optical networks. International Journal of Autonomous and Adaptive Communications Systems, 1(2):220-241, 2008 .

[458] T. Sowle, N. Gardini, F.V.A. Vazquez, E. Pérez, J.A. Jimenez, and L. DePagter. A simulation-IP based tool for patient admission services in a multi-specialty outpatient clinic. In Proceedings of the Winter Simulation Conference, pages 1186-1197, Savannah, USA, 2014.

[459] M.G. Speranza and Z. Tuza. On-line approximation algorithms for scheduling tasks on identical machines with extendable working time. Annals of Operations Research, 86(0):491-506, 1999.

[460] R. Spliet and G. Desaulniers. The discrete time window assignment vehicle routing problem. European Journal of Operational Research, 244(2):379-391, 2015.

[461] A. Stanciu, L. Vargas, and J. May. A revenue management approach for managing operating room capacity. In Proceedings of the Winter Simulation Conference, pages 2444-2454, Baltimore, USA, 2010.

[462] C.R. Standridge and D. Steward. Using expert systems for simulation modeling of patient scheduling. Simulation, 75(3):148-156, 2000. 
[463] T. Stevens, M. De Leenheer, C. Develder, B. Dhoedt, K. Christodoulopoulos, P. Kokkinos, and E. Varvarigos. Multi-cost job routing and scheduling in grid networks. Future Generation Computer Systems, 25(8):912-925, 2009.

[464] D.P. Strum, L.G. Vargas, and J.H. May. Surgical subspecialty block utilization and capacity planning: a minimal cost analysis model. Anesthesiology, 90(4): 1176-1185, 1999.

[465] S. Su and C.L. Shih. Managing a mixed-registration-type appointment system in outpatient clinics. International Journal of Medical Informatics, 70(1):31-40, 2003.

[466] A. Sulistio, K.H. Kim, and R. Buyya. On incorporating an on-line strip packing algorithm into elastic grid reservation-based systems. In Proceedings of the International Conference on Parallel and Distributed Systems, volume 2, Hsinchu, Taiwan, 2007.

[467] A. Sulistio, K.H. Kim, and R. Buyya. Managing cancellations and no-shows of reservations with overbooking to increase resource revenue. In Proceedings of the 8th IEEE International Symposium on Cluster Computing and the Grid, pages 267-276, Lyon, France, 2008.

[468] R.W. Swain, K.E. Kilpatrick, and J.J. Marsh. Implementation of a model for census prediction and control. Health Services Research, 12(4):380-395, 1977.

[469] Z. Tan and A. Zhang. Online and semi-online scheduling. In P.M. Pardalos, D.Z. Du, and R.L. Graham, editors, Handbook of Combinatorial Optimization, volume 4, pages 2191-2252. Springer Science+Business Media, New York, USA, second edition, 2013.

[470] J. Tang, C. Yan, and R.Y.K. Fung. Optimal appointment scheduling with noshows and exponential service time considering overtime work. Journal of Management Analytics, 1(2):99-129, 2014.

[471] G.J. Taylor, S.I. McClean, and P.H. Millard. Stochastic models of geriatric patient bed occupancy behaviour. Journal of the Royal Statistical Society: Series A (Statistics in Society), 163(1):39-48, 2000.

[472] E. Tekin and I. Sabuncuoglu. Simulation optimization: A comprehensive review on theory and applications. IIE Transactions, 36(11):1067-1081, 2004.

[473] A. Testi, E. Tanfani, and G. Torre. A three-phase approach for operating theatre schedules. Health Care Management Science, 10(2):163-172, 2007.

[474] The editors. Looking back on the millennium in medicine. New England Journal of Medicine, 342(13):988-988, 2000.

[475] The Health Care Inspectorate. Kwaliteitsindicatoren: basisset ziekenhuizen 2015 [in Dutch]. Booklet (available at www.igz.nl), Utrecht, September 2014.

[476] M. Thomas and H. Szczerbicka. Online scheduling on multiple resources under stochastic conditions. Systems Science, 33(2):23-30, 2007. 
[477] M.S. Thomsen and O. Nørrevang. A model for managing patient booking in a radiotherapy department with differentiated waiting times. Acta Oncologica, 48 (2):251-258, 2009.

[478] L. Tomás, C. Carrión, B. Caminero, and A. Caminero. Meta-scheduling in advance using red-black trees in heterogeneous grids. In Proceedings of the IEEE International Parallel \& Distributed Processing Symposium, Atlanta, USA, 2010.

[479] V. Toporkov, A. Toporkova, A. Tselishchev, and D. Yemelyanov. Slot selection algorithms in distributed computing. The Journal of Supercomputing, 69(1):5360, 2014.

[480] J. Triay, C. Cervelló-Pastor, and V.M. Vokkarane. Analytical blocking probability model for hybrid immediate and advance reservations in optical WDM networks. IEEE/ACM Transactions on Networking, 21(6):1890-1903, 2013.

[481] P.M. Troy and L. Rosenberg. Using simulation to determine the need for ICU beds for surgery patients. Surgery, 146(4):608-617, 2009.

[482] V.A. Truong. Optimal advance scheduling. Management Science, 61(7):15841597, 2015.

[483] P.F.J. Tsai and G.Y. Teng. A stochastic appointment scheduling system on multiple resources with dynamic call-in sequence and patient no-shows for an outpatient clinic. European Journal of Operational Research, 239(2):427-436, 2014.

[484] A. Turkcan, B. Zeng, K. Muthuraman, and M. Lawley. Sequential clinical scheduling with service criteria. European Journal of Operational Research, 214(3):780$795,2011$.

[485] R. Umar, A. Agarwal, and C.R. Rao. Advance planning and reservation in a grid system. In R. Benlamri, editor, Networked Digital Technologies, volume 293 of Communications in Computer and Information Science, pages 161-173. SpringerVerlag, Berlin Heidelberg, Germany, 2012.

[486] United States Census Bureau. Monthly population estimates for the united states: April 1, 2000 to december 1, 2010 (na-est2009-01). Database (available at www.census.gov).

[487] M. Utley, S. Gallivan, K. Davis, P. Daniel, P. Reeves, and J. Worrall. Estimating bed requirements for an intermediate care facility. European Journal of Operational Research, 150(1):92-100, 2003.

[488] M. Utley, S. Gallivan, T. Treasure, and O. Valencia. Analytical methods for calculating the capacity required to operate an effective booked admissions policy for elective inpatient services. Health Care Management Science, 6(2):97-104, 2003 .

[489] M. Utley, S. Gallivan, and M. Jit. How to take variability into account when planning the capacity for a new hospital unit. In J. Vissers and R. Beech, editors, Health Operations Management: Patient Flow Logistics in Health Care. Routledge, 2005. 
[490] J. Van den Bergh, J. Beliën, P. De Bruecker, E. Demeulemeester, and L. De Boeck. Personnel scheduling: a literature review. European Journal of Operational Research, 226(3):367 - 385, 2013.

[491] P.T. Vanberkel and J. Blake. A comprehensive simulation for wait time reduction and capacity planning applied in general surgery. Health Care Management Science, 10:373-385, 2007.

[492] P.T. Vanberkel, R.J. Boucherie, E.W. Hans, J.L. Hurink, and N. Litvak. A survey of health care models that encompass multiple departments. International Journal of Health Management and Information, 1(1):37-69, 2009.

[493] P.T. Vanberkel, R.J. Boucherie, E.W. Hans, J.L. Hurink, W.A.M. van Lent, and W.H. van Harten. An exact approach for relating recovering surgical patient workload to the master surgical schedule. Journal of the Operational Research Society, 62(10):1851-1860, 2011.

[494] W. Vancroonenburg, P. De Causmaecker, and G. Vanden Berghe. A study of decision support models for online patient-to-room assignment planning. Annals of Operations Research, 239(1):253-271, 2016.

[495] E. Varvarigos, V. Sourlas, and K. Christodoulopoulos. Routing and scheduling connections in networks that support advance reservations. Computer Networks, 52(15):2988-3006, 2008.

[496] E.A. Varvarigos and V. Sharma. An efficient reservation connection control protocol for gigabit networks. Computer Networks and ISDN Systems, 30(12):11351156, 1998.

[497] C. Vasilakis and E. El-Darzi. A simulation study of the winter bed crisis. Health Care Management Science, 4(1):31-36, 2001.

[498] C. Vasilakis, E. El-Darzi, and P. Chountas. A decision support system for measuring and modelling the multi-phase nature of patient flow in hospitals. In P. Chountas, I. Petrounias, and J. Kacprzyk, editors, Intelligent Techniques and Tools for Novel System Architectures, volume 109 of Studies in Computational Intelligence, chapter 12, pages 201-217. Springer Berlin Heidelberg, 2008.

[499] M. Vasirani and S. Ossowski. Learning and coordination for autonomous intersection control. Applied Artificial Intelligence, 25(3):193-216, 2011.

[500] R. Velásquez, T. Melo, and K.H. Küfer. Tactical operating theatre scheduling: efficient appointment assignment. In J. Kalcsics and S. Nickel, editors, Operations Research Proceedings 200\%, pages 303-308. Springer-Verlag, Berlin Heidelberg, Germany, 2008.

[501] M.F. van der Velde, N. Kortbeek, and N. Litvak. Organizing multidisciplinary care for children with neuromuscular diseases. Working paper (available at www.math.utwente.nl/publications), University of Twente, Enschede, the Netherlands, 2012. 
[502] I. Vermeulen, S. Bohte, S. Elkhuizen, P. Bakker, and H. La Poutré. Decentralized online scheduling of combination-appointments in hospitals. In J. Rintanen, B. Nebel, J.C. Beck, and E. Hansen, editors, Proceedings of the 18th International Conference on Automated Planning and Scheduling, pages 372-379, California, USA, 2008. The AAAI Press.

[503] I.B. Vermeulen, S.M. Bohte, P.A.N. Bosman, S.G. Elkhuizen, P.J.M. Bakker, and J.A. La Poutré. Optimization of online patient scheduling with urgencies and preferences. In C. Combi, Y. Shahar, and A. Abu-Hanna, editors, Artificial Intelligence in Medicine, volume 5651 of Lecture Notes in Computer Science, pages 71-80. Springer-Verlag, Berlin Heidelberg, Germany, 2009.

[504] I.B. Vermeulen, S.M. Bohte, S.G. Elkhuizen, H. Lameris, P.J.M. Bakker, and H. La Poutré. Adaptive resource allocation for efficient patient scheduling. Artificial Intelligence in Medicine, 46(1):67-80, 2009.

[505] W. Vink, A. Kuiper, B. Kemper, and S. Bhulai. Optimal appointment scheduling in continuous time: the lag order approximation method. European Journal of Operational Research, 240(1):213-219, 2015.

[506] J.T. Virtamo. A model of reservation systems. IEEE Transactions on Communications, 40(1):109-118, 1992.

[507] J.T. Virtamo and S. Aalto. Stochastic optimization of reservation systems. European Journal of Operational Research, 51(3):327-337, 1991.

[508] J.M.H. Vissers, I.J.B.F. Adan, and J.A. Bekkers. Patient mix optimization in tactical cardiothoracic surgery planning: a case study. IMA Journal of Management Mathematics, 16(3):281-304, 2005.

[509] J. de Vreede. Kennemer gasthuis nummer 1 in ad ziekenhuis top 100 [in Dutch]. Newspaper item (available at www.ad.nl), 13-11-2015.

[510] N.M. van de Vrugt, N. Litvak, and R.J. Boucherie. Blocking probabilities in Erlang loss queues with advance reservation. Stochastic Models, 30(2):187-196, 2014 .

[511] D.J. Wallace, D.C. Angus, C.W. Seymour, A.E. Barnato, and J.M. Kahn. Critical care bed growth in the united states. a comparison of regional and national trends. American Journal of Respiratory and Critical Care Medicine, 191(4):410-416, 2014.

[512] T. Wang, A. Guinet, and B. Besombes. A sizing tool for allocation planning of hospital bed resources. In S. McClean, P. Millard, E. El-Darzi, and C. Nugent, editors, Intelligent Patient Management, volume 189 (Part 2) of Studies in Computational Intelligence, pages 113-125. Springer-Verlag, Berlin Heidelberg, Germany, 2009.

[513] T. Wang, E. Marcon, P. Pommier, and T. Zhang. Decision support tool for patient recruitment in a hadrontherapy center. In S. Bittanti, A. Cenedese, and S. Zampieri, editors, Proceedings of the 18th International Federation of Automatic Control World Congress, volume 18, pages 10416-10421, Milano, Italy, 2011. International Federation of Automatic Control. 
[514] W.Y. Wang and D. Gupta. Adaptive appointment systems with patient preferences. Manufacturing \& Service Operations Management, 13(3):373-389, 2011.

[515] C.A. White and G.M. White. Scheduling doctors for clinical training unit rounds using tabu optimization. In E. Burke and P. De Causmaecker, editors, Practice and Theory of Automated Timetabling IV, volume 2740 of Lecture Notes in Computer Science, pages 120-128. Springer Berlin Heidelberg, 2003.

[516] W. Whitt. The queueing network analyzer. Bell System Technical Journal, 62 (9):2779-2815, 1983.

[517] J.L. Wiler, R.T. Griffey, and T. Olsen. Review of modeling approaches for emergency department patient flow and crowding research. Academic Emergency Medicine, 18(12):1371-1379, 2011.

[518] J. Williams, S. Dumont, J. Parry-Jones, I. Komenda, J. Griffiths, and V. Knight. Mathematical modelling of patient flows to predict critical care capacity required following the merger of two district general hospitals into one. Anaesthesia, 70 (1):32-40, 2015.

[519] W.L. Winston. Operations research: applications and algorithms. Brooks/Cole Thomson Learning, Belmont, USA, fourth edition, 2003.

[520] World Health Organization. Health and development. Accessed December 2015 (available at www.who.int/hdp).

[521] S. Wu and D. Kotak. Agent-based collaborative project management system for distributed manufacturing. In Proceedings of the IEEE International Conference on Systems, Man 83 Cybernetics, volume 2, pages 1223-1228, Washington, USA, 2003.

[522] X.D. Wu, M.T. Khasawneh, D.M. Yue, Y.N. Chu, and Z.T. Gao. A simulation study of outpatient scheduling with multiple providers and a single device. International Journal of Computational Intelligence Systems, 7(supplement 2):15-25, 2014.

[523] P. Xiao, P. Qu, and X. Qu. An adaptive redundant reservation strategy in distributed high-performance computing environments. International Journal of Hybrid Information Technology, 6(6):51-64, 2013.

[524] J. Xu, X. Xu, and S. Q. Xie. Recent developments in dual resource constrained (DRC) system research. European Journal of Operational Research, 215(2):309318, 2011.

[525] C. Yan, J. Tang, and B. Jiang. Sequential appointment scheduling considering walk-in patients. Mathematical Problems in Engineering, page Article ID 564832, 2014.

[526] K.K. Yang, M.L. Lau, and S.A. Quek. A new appointment rule for a single-server, multiple-customer service system. Naval Research Logistics, 45(3):313-326, 1998.

[527] M. Yang and X. Lu. On-line and semi-online scheduling for flow shop problems on two machines. Optimization, 62(4):499-507, 2013. 
[528] M. Yang, M.J. Fry, J. Raikhelkar, C. Chin, A. Anyanwu, J. Brand, and C. Scurlock. A model to create an efficient and equitable admission policy for patients arriving to the cardiothoracic ICU. Critical Care Medicine, 41(2):414-422, 2013.

[529] R. Yang, S. Bhulai, R. van der Mei, and F. Seinstra. Optimal resource allocation for time-reservation systems. Performance Evaluation, 68(5):414-428, 2011.

[530] Y. Yang, W. Jin, and X. Hao. Car rental logistics problem: a review of literature. In Proceedings of the IEEE International Conference on Service Operations and Logistics, and Informatics, volume 2, pages 2815-2819, Beijing, China, 2008.

[531] D. Ye and G. Zhang. On-line scheduling with extendable working time on a small number of machines. Information Processing Letters, 85(4):171-177, 2003.

[532] G.B. Yom-Tov and A. Mandelbaum. Erlang-R: a time-varying queue with reentrant customers, in support of healthcare staffing. Manufacturing $\&$ Service Operations Management, 16(2):283-299, 2014.

[533] H.J. Yoon and D.Y. Lee. Online scheduling of integrated single-wafer processing tools with temporal constraints. IEEE Transactions on Semiconductor Manufacturing, 18(3):390-398, 2005.

[534] L. Yuan, C.K. Tham, and A.L. Ananda. A probing approach for effective distributed resource reservation. In M.A. Marsan, G. Corazza, M. Listanti, and A. Roveri, editors, Quality of Service in Multiservice IP Networks, volume 2601 of Lecture Notes in Computer Science, pages 672-688. Springer-Verlag, Berlin Heidelberg, Germany, 2003.

[535] C. Zacharias and M. Armony. Joint panel sizing and appointment scheduling in outpatient care. Working paper (available at people.stern.nyu.edu/cz464), Stern School of Business, New York University, New York, USA, 2015.

[536] C. Zacharias and M. Pinedo. Managing customer arrivals in service systems with multiple servers. Working paper (available at ssrn.com), Stern School of Business, New York University, New York, USA, 2014.

[537] C. Zacharias and M. Pinedo. Appointment scheduling with no-shows and overbooking. Production and Operations Management, 23(5):788-801, 2014.

[538] G. Zayas-Caban, J. Xie, L. Green, and M.E. Lewis. Optimal control of an emergency room triage and treatment process. Working paper (available at ssrn.com) Paper No. 14-51, Columbia Business School Research, September 2014.

[539] B. Zeng, A. Turkcan, J. Lin, and M. Lawley. Clinic scheduling models with overbooking for patients with heterogeneous no-show probabilities. Annals of Operations Research, 178(1):121-144, 2010.

[540] A. Zhang, Y. Jiang, L. Fan, and J. Hu. Optimal online algorithms on two hierarchical machines with tightly-grouped processing times. Journal of Combinatorial Optimization, 29(4):781-795, 2015. 
[541] B. Zhang, P. Murali, M.M. Dessouky, and D. Belson. A mixed integer programming approach for allocating operating room capacity. Journal of the Operational Research Society, 60(5):663-673, 2009.

[542] C. Zhao, J. Cao, H. Wu, and F. Zhang. Cost estimation of advance reservations over queued jobs: a quantitative study. International Journal of Modeling, Simulation, and Scientific Computing, 1(3):317-332, 2010.

[543] Y. Zhao, K. Triantis, D. Teodorović, and P. Edara. A travel demand management strategy: the downtown space reservation system. European Journal of Operational Research, 205(3):584-594, 2010.

[544] G. Zhu, D. Lizotte, and J. Hoey. Scalable approximate policies for Markov decision process models of hospital elective admissions. Artificial Intelligence in Medicine, 61(1):21-34, 2014.

[545] L. Zhu, N. Geng, and X. Xie. MDP-based appointment scheduling for one examination of two types of outpatients. In Proceedings of the IEEE International Conference on Automation Science and Engineering, pages 764-769, Madison, USA, 2013.

[546] X. Zhu and M. Veeraraghavan. Analysis and design of book-ahead bandwidthsharing mechanisms. IEEE Transactions on Communications, 56(12):2156-2165, 2008.

[547] Z. Zhu. Impact of different discharge patterns on bed occupancy rate and bed waiting time: a simulation approach. Journal of Medical Engineering 83 Technology, 35(6-7):338-343, 2011.

[548] Z. Zhu. An online short-term bed occupancy rate prediction procedure based on discrete event simulation. Journal of Hospital Administration, 3(4):p37, 2014.

[549] M.E. Zonderland and R.J. Boucherie. Queuing networks in health care systems. In R. Hall, editor, Handbook of Healthcare System Scheduling, volume 168 of International Series in Operations Research 83 Management Science, chapter 9, pages 201-243. Springer Science+Business Media, Dordrecht, the Netherlands, 2012 .

[550] M.E. Zonderland, F. Boer, R.J. Boucherie, A. de Roode, and J.W. van Kleef. Redesign of a university hospital preanesthesia evaluation clinic using a queuing theory approach. Anesthesia \& Analgesia, 109(5):1612-1621, 2009.

[551] M.E. Zonderland, R.J. Boucherie, and A. Al Hanbali. Appointments in care pathways: the $G_{e o}^{x} / D / 1$ queue with slot reservations. Queueing Systems, 79(1): $37-51,2015$.

[552] M.E. Zonderland, R.J. Boucherie, M.W. Carter, and D.A. Stanford. Modeling the effect of short stay units on patient admissions. Operations Research for Health Care, 5:21-27, 2015.

[553] L. Zuo, M.M. Zhu, and C.Q. Wu. Fast and efficient bandwidth reservation algorithms for dynamic network provisioning. Journal of Network and Systems Management, 23(3):420-444, 2015. 
[554] S. Zurheide and K. Fischer. Revenue management methods for the liner shipping industry. Flexible Services and Manufacturing Journal, 27(2-3):200-223, 2015. 
1-1 One appointment on one resource type

1-m One appointment on multiple resource types

ABM Agent based model

AMU Acute medical unit

ANN Artificital neural network

cap Capacity allocation

CHOIR Center for Healthcare Operations Improvement and Research

DES Discrete event simulation

ED Emergency department

EF Existing patients first

FCFS First come first served

GOL Greedy online

GP General practitioner

ICU Intensive care unit

ILP Integer linear programming

JBH Jeroen Bosch hospital

JBZ Jeroen Bosch ziekenhuis

LoS Length of stay

LS Local search

m-1 Multiple appointments on one resource type

MDM Multi disciplinary meeting

MDP Markov decision process

MIP Mixed integer programming

m-m Multiple appointments on multiple resource types

MOL Modified offered load

MRI Magnetic Resonance Imaging

MSS Master surgery schedule 
near Near-online appointment scheduling

NF New patients first

NP Nurse practitioner

NR Next room first

OBS Obstetric ward

onl Online appointment scheduling

OR Operations research

OT Operating theater

QNA Queueing network analyzer

RPC Working routine and room assignment policy combination

RND Random patient next

SA Simulated annealing

SEH Spoedeisende hulp-afdeling

SMIP Stochastic mixed integer programming

WDW Weekday ward

WI Walk-in 


\section{Summary}

Despite the long experienced urgency of rapidly increasing healthcare expenditures, there is still a large potential to improve hospitals' logistical efficiency. Operations Research (OR) methodologies may support healthcare professionals in making better decisions concerning planning and capacity issues and improving efficiency in the delivery of healthcare, but appears to be lacking implementation in practice. This thesis displays OR research that focuses both on theoretical results and practical applications, aiming to improve efficiency while improving compliance with patients' and physicians' preferences.

\section{Part I Operations Research applied to hospital wards}

Hospital wards affect patient flow through the hospital, as the availability of beds determines if new patients are admitted or may undergo surgery. Part I focuses on OR methodologies applied to hospital wards and their integration into practice.

Chapter 2 first defines the different OR methodologies applied to hospital wards, and the logistical performance measures considered: blocking probability, utilization and throughput. Next, we review the OR literature applied to hospital wards. Based on logistical characteristics and common patient flow problems, we distinguish the following particular ward types: intensive care, acute medical units, obstetric wards, weekday wards, and general wards. We analyze typical trade-offs of performance measures for each ward type, and the common OR models applied to it. Additionally, we provide four modeling examples, discuss reported experiences with implementation of the research, and highlight voids in the literature that may be directions for future research. Main conclusions of the review are: there exist many papers on OR models relating to different types of wards, actual use of the models in practice seems scarce, and the stakeholders of a project play a significant role in the likelihood of implementation. Additionally, researchers should be thorough in their data collection, sensitivity and robustness analyses, and implementation support.

Chapter 3 contains an implementation-oriented case study in which we invoke both queueing theory and discrete event simulation, applied to medical wards of the Jeroen Bosch Hospital (JBH) that experienced unbalanced bed occupancies. In this case study, medical specialists proposed interventions. The effects of all possible interventions were quantified in advance using OR methods, which enabled the steering group to choose the most promising intervention to implement. This intervention was supported by all involved staff, and proved to be effective in reality. 


\section{Part II Online appointment scheduling}

Online appointment scheduling refers to systems in which customers receive an immediate answer to their appointment request. This answer is either an appointment time, or a refusal. In an online system, a scheduler has no information about the future when he has to decide on the current request. Therefore, offline systems, in which the scheduler collects all requests before making the decisions, often achieve higher utilization and acceptance rates.

Chapter 4 provides an extensive literature review of online appointment scheduling for different application areas, not limited to healthcare. The literature is categorized according to the number of appointments each customer requires, the number of resource types at the facility, and the horizon at which the scheduling decisions are made. We provide an overview of the scheduling decisions, the objectives, and the OR methods applied in different application areas. Different application areas focus on different modeling assumptions and goals, but in all application areas, there is a continuous trade-off between customerfriendliness, for example, short access and waiting times, and system efficiency, for example, little idle time and overtime of the server. Most online appointment scheduling literature considers systems with one resource in which customers make a single appointment. We highlight gaps in the literature that represent opportunities for future research.

In Chapter 5 we study the effect of introducing advance reservation, i.e., an online appointment system instead of a walk-in system, on the blocking probability for a general queueing model. It appears that introducing advance reservation may increase or decrease the blocking probability, depending on the system parameters. The lower blocking probabilities are achieved because systems with advance reservation tend to accept many relatively short jobs.

\section{Part III Patient appointment schedules}

Patient appointment schedules determine to a large extent patients' access and waiting times. Part III entails two chapters in which we optimize patient appointment schedules to improve patient flow and reduce specialists' idle time and overtime for two JBH departments.

At the breast center of the $\mathrm{JBH}$, patients undergo a series of diagnostic tests on different machines to obtain a diagnose. In Chapter 6 we apply a discrete time queueing model to determine the necessary capacity to comply with the national access time norms. The center implemented interventions based on the model, which concern a new patient appointment schedule and an additional multidisciplinary meeting. We show that the interventions reduce both the appointment and diagnostics delay. Additionally, we propose a promising new patient appointment schedule to further reduce patient waiting times and staff overtime, based on a discrete event simulation. It appears that waiting and overtime may be reduced if patient types are not clustered in the schedule but spread over the day, and idle time is divided equally over the day instead of clustered at the end of the day. 
Chapter 7 presents an implementation-oriented case study in which we investigate different appointment scheduling policies for the JBH plaster room. At the plaster room patients may either make an appointment or walk-in. Invoking discrete event simulation, we investigate different appointment slot lengths and times, different proportions of patients making appointments instead of walking in, and different arrival rate scenarios. It appears from the simulation results that waiting and overtime are acceptable for most patients and workdays, but exceptionally high values occur for both performance measures. We show that some appointment rules are both easy to implement, and promising to reduce overtime while having similar or less waiting time.

\section{Part IV Optimizing doctor schedules}

Patient appointment schedules build upon specialists' schedules, as appointment can only be scheduled at times that the specialists are available. Part IV focuses on optimizing specialist schedules, to improve compliance with both specialists' preferences and patients' access times norms.

In Chapter 8 we optimize the schedule of gynecologists. Gynecologists, and specialists in general, typically have many different tasks, such as seeing outpatients, on call duties, and performing surgeries. Due to specializations, each task may only be performed by a subset of the gynecologists. There are many hard and soft constraints on the sequence and frequency of tasks in a schedule. We invoke mixed integer linear programming to optimize the assignment of tasks to gynecologists and shifts. For practical purposes, we investigate two heuristics that appear to be very effective in swiftly generating good schedules. The resulting roster not only reduces the time to obtain a roster, but also compliance with specialists' preferences and patients' access times.

Chapter 9 provides a quantification of the benefits of scheduling outpatient clinic hours dynamically, which entails that part of the capacity is only scheduled in case the access times exceed a certain threshold. The optimal dynamic scheduling policy is obtained through a Markov decision problem. We compare the dynamic schedule to the optimal static schedule, which is obtained invoking an integer linear program. Both methods are applied to the Surgery department of the JBH. Although the precise outcomes are parameter specific, dynamic scheduling significantly improves system utilization and access time norm compliance.

\section{Part V Optimizing throughput at the ED}

Emergency departments (EDs) often experience severe overcrowding, which may put patient lives at risk. Typically, specialists at EDs use multiple rooms in parallel; while one patient awaits test results in a treatment room, the specialist visits other patients. The assignment of rooms among the specialists is often unbalanced, which affects the blocking probability, waiting time and length of stay of patients.

In Chapter 10 we analyze patients' expected sojourn times invoking a queue- 
ing model in a random environment, for different room assignment policies and working routines of the specialists. We conduct a discrete event simulation to validate our model in case of time-varying arrivals, which are typical for EDs. It appears that a specialist should always be assigned at least two rooms in parallel. Additionally, if a specialist has to choose between seeing a new patient or visiting one of the patients waiting for their test results, the specialist should always take the first option.

We extend the research of Chapter 10 in Chapter 11 to incorporate more realistic assumptions, such as time-varying arrivals and patient- and physiciantype dependent treatment times. To this end, we invoke a mixed integer programming model in a rolling horizon approach. Additionally, we analyze several extreme cases of the ED system to investigate which policy results in the best performance for which parameter setting. For the realistic ED system, numerical results indicate that giving priority to existing patients results in the best performance. As this policy prescribes the exact opposite compared to the results of Chapter 10, we conclude that it is unlikely that there exists a policy that is optimal for all ED systems.

\section{Conclusions}

Optimizing the efficiency of healthcare logistics often improves both patientfriendliness and quality of care through better accessibility and alignment of the appointments, and may provide the hospital with the possibility to treat more patients with the same capacity. This thesis provides both theoretical and pragmatic applications of OR methodologies that support logistical decision making in hospitals. The research in this thesis contributes to the vast amount of academic papers on OR in healthcare, and additionally provides implementationoriented case studies and experiences with implementing research results. We focus on inventive, pragmatic mathematical solutions with a human touch, by incorporating both patient and physician preferences in our approaches. With this thesis we intend to bring theory and practice closer together, so academics and practitioners can join forces in the continuous improvement of the efficiency of healthcare logistics. 


\section{Samenvatting}

Er is veel potentie om de logistieke efficiëntie van ziekenhuizen te verbeteren, ondanks dat de urgentie van sterk stijgende zorgkosten inmiddels algemeen bekend is. Methodieken uit Operationele Besliskunde (in het Engels 'Operations Research, afgekort tot OR) kunnen zorgprofessionals ondersteunen bij het maken van betere beslissingen bij planning- en capaciteitsproblemen en het verbeteren van de logistieke efficiëntie in het verlenen van zorg. Desondanks blijken veel onderzoeksresultaten niet geïmplementeerd te worden in de zorgpraktijk. Dit proefschrift bevat OR onderzoek gericht op zowel de theoretische resultaten als praktische toepassingen, waarin naast efficiëntie ook de wensen van patiënten en specialisten centraal staan.

\section{Deel I OR toegepast op verpleegafdelingen}

Verpleegafdelingen beïnvloeden de patiëntenstromen door een ziekenhuis, omdat de beschikbaarheid van bedden bepaalt of nieuwe patiënten worden opgenomen of een operatie mogen ondergaan. Deel I belicht OR methodieken die toegepast zijn op verpleegafdelingen in ziekenhuizen, en de implementatie van deze methodes in de praktijk.

Hoofdstuk 2 duidt eerst zowel de verschillende OR methodieken die toegepast worden op verpleegafdelingen, als de belangrijkste logistieke kwaliteitsindicatoren: blokkeringskans, bezettingsgraad en doorstroom. Daarna geven we een overzicht van de OR literatuur die toegepast is op verpleegafdelingen. We onderscheiden verschillende typen verpleegafdelingen op basis van kenmerkende logistieke eigenschappen en problemen: intensive care, acute opnameafdeling, obstetrie, kortverblijf en overige verpleegafdelingen. Voor ieder type verpleegafdeling analyseren we zowel typische afwegingen tussen verschillende kwaliteitsindicatoren, als de meest toegepaste OR modellen. Vervolgens geven we vier voorbeelden van toegepaste OR modellen, bespreken we gerapporteerde ervaringen met implementatie van onderzoeksresultaten in de praktijk en belichten we hiaten in de literatuur die mogelijk toekomstige onderzoeksrichtingen zijn. De belangrijkste conclusies van het literatuuroverzicht zijn: er is veel literatuur over OR modellen die toegepast zijn op verschillende typen verpleegafdelingen, daadwerkelijk gebruik van de modellen in de praktijk lijkt zeldzaam en de belanghebbenden van een project hebben een significante rol in de implementatiekansen van het onderzoek. Ook moeten onderzoekers zorgvuldig hun data verzamelen, de sensitiviteits- en robuustheidsanalyse uitvoeren en ondersteuning bieden bij implementatie.

Hoofdstuk 3 beschrijft een praktisch adviesproject waarin we zowel wachtrijtheorie als simulatie toepassen op beschouwende verpleegafdelingen van het 
Jeroen Bosch Ziekenhuis (JBZ) die kampten met ongebalanceerde bedbezettingen. In deze casestudy hebben medisch specialisten de interventies aangedragen. Deze interventies zijn prospectief cijfermatig geanalyseerd door middel van OR modellen, waardoor de stuurgroep de meest veelbelovende interventie heeft geselecteerd voor implementatie. Deze interventie is onderschreven door alle betrokken personeelsleden en blijkt effectief in de praktijk.

\section{Deel II Online afsprakenplanning}

Online afsprakenplanning refereert naar systemen waarin klanten onmiddellijk antwoord krijgen op hun afspraakverzoek. Dit antwoord is ofwel een afspraaktijd, of een afwijzing. In online systemen heeft een serviceverlener geen informatie over de toekomst wanneer hij een beslissing moet maken over het huidige afspraakverzoek. Daarom kunnen offline systemen, waarin de serviceverlener alle afspraakverzoeken verzamelt voordat hij beslissingen maakt, vaak een hogere bezettingsgraad en acceptatiekans realiseren.

Hoofdstuk 4 bevat een uitgebreid literatuuronderzoek naar online afspraaksystemen voor verschillende toepassingsgebieden, niet beperkt tot de zorg. De literatuur is gecategoriseerd op basis van het aantal afspraken in ieder afspraakverzoek, het aantal typen middelen (bijvoorbeeld personele middelen of apparatuur) van de serviceverlener en de planningshorizon waarop de beslissingen gemaakt worden. We geven een overzicht van de planningsbeslissingen, planningsdoelen en de toegepaste OR methodes voor verschillende toepassingsgebieden. Hoewel verschillende toepassingsgebieden focussen op verschillende modelaannames en -doelstellingen, is er in alle toepassingsgebieden een continue afweging tussen enerzijds klantvriendelijkheid, bijvoorbeeld lage toegangs- en wachttijden, en anderzijds efficiëntie, bijvoorbeeld weinig leegstand en overwerktijd. Literatuur over online afsprakenplanning richt zich vooral op systemen met één type middelen, waarin afspraakverzoeken slechts één afspraak bevatten en de serviceverlener direct moet antwoorden op ieder afspraakverzoek. We noemen onderwerpen die onderbelicht zijn in de literatuur en veelbelovend zijn voor toekomstige onderzoeksrichtingen.

In Hoofdstuk 5 bestuderen we het effect van het introduceren van reserveringen in een systeem waarin klanten op inloop geholpen worden. Uit dit onderzoek blijkt dat het introduceren van reserveringen de blokkeringskans laat stijgen of dalen, afhankelijk van de parameters van het systeem. Een reductie in de blokkeringskans wordt gerealiseerd doordat systemen met reserveringen relatief veel korte afspraken accepteren.

\section{Deel III Optimaliseren van afsprakenschema's voor patiënten}

Afsprakenschema's bepalen voor een groot deel de toegangs- en wachttijd voor patiënten. Deel III bevat twee hoofdstukken waarin we afsprakenschema's optimaliseren voor twee afdelingen van het JBZ, met als doel om zowel de patiëntenstromen te verbeteren, als de onbenutte tijd en overtijd voor specialisten te reduceren. 
Voordat patiënten van het Borstcentrum van het JBZ een diagnose krijgen, moeten zij een serie diagnostische tests ondergaan. In Hoofdstuk 6 bepalen we de benodigde capaciteit om aan de nationale normen op de toegangstijd te voldoen, met behulp van een discrete tijd wachtrijmodel. Het Borstcentrum heeft naar aanleiding van de analyse een nieuw afsprakenschema en een extra multidisciplinair overleg geïmplementeerd. We tonen aan dat de interventies zowel de toegangstijd als de tijd tot de definitieve diagnose reduceren. Daarnaast presenteren we een veelbelovend nieuw afsprakenschema om de wachttijd van patiënten en overtijd van personeel te reduceren, gebaseerd op de resultaten van een zogenaamde 'discrete event' simulatie. Uit de simulatie blijkt dat de wacht- en overwerktijd gereduceerd kan worden door patiënttypes en ingeplande uitlooptijd niet te groeperen in het afsprakenschema, maar ze te verspreiden over de dag.

Hoofdstuk 7 beschrijft een praktisch adviesproject waarin we verschillende afsprakenschema's onderzoeken voor de Gipskamer van het JBZ. De Gipskamer werkt zowel op afspraak, als op inloopbasis. Met behulp van discrete event simulatie onderzoeken we verschillende afspraaklengtes en -tijden, verschillende verhoudingen tussen afspraak en inloop en verschillende aankomstintensiteiten. Uit de simulatieresultaten blijkt dat de wacht- en overwerktijd voor de meeste patiënten en werkdagen acceptabel zijn, maar dat uitzonderlijk hoge waarden voorkomen voor beide prestatie-indicatoren. We tonen aan dat enkele afspraakregels zowel eenvoudig te implementeren zijn, als veelbelovend voor het reduceren van de overwerktijd voor gelijke of kortere wachttijden.

\section{Deel IV Optimaliseren van artsenagenda's}

Afsprakenschema's voor patiënten zijn afhankelijk van artsenagenda's, omdat afspraken met patiënten alleen ingepland kunnen worden wanneer de arts daarvoor beschikbaar is. Deel IV is gericht op het optimaliseren van artsenagenda's om meer rekening te houden met de wensen van artsen en beter te voldoen aan toegangstijdnormen.

In Hoofdstuk 8 optimaliseren we het rooster van gynaecologen. Gynaecologen, en artsen in het algemeen, hebben vaak veel verschillende taken, zoals het zien van patiënten op de poli, draaien van diensten en opereren van patiënten. Iedere gynaecoloog heeft haar eigen subspecialisatie, waardoor iedere taak slechts door een deelverzameling van de gynaecologen uitgevoerd mag worden. Er zijn verschillende harde en zachte eisen waaraan de volgorde en frequentie van taken in een rooster moet voldoen. We stellen een geheeltallig lineair programmeringsmodel op om het toewijzen van taken aan dagdelen en gynaecologen te optimaliseren. Vanwege praktische overwegingen onderzoeken we twee heuristieken, die beiden heel effectief blijken te zijn in het snel genereren van goede roosters. Met dit onderzoek reduceren we niet alleen de tijd om een rooster te maken, maar voldoet het rooster ook beter aan de wensen en eisen van de gynaecologen en verbetert de toegangstijd van patiënten.

Hoofdstuk 9 bevat een kwantificering van de voordelen van dynamisch 
plannen van poli-capaciteit, wat betekent dat een deel van de beschikbare capaciteit slechts wordt ingezet wanneer de toegangstijden boven een bepaalde grens komen. De optimale politiek voor dynamisch roosteren is verkregen door middel van Markov beslissingstheorie. Het optimale dynamische rooster vergelijken we met het optimale statische rooster, dat verkregen is met behulp van een geheeltallig lineair programmeringsmodel. Beide methoden zijn toegepast op de polikliniek chirurgie van het JBZ. Hoewel de precieze uitkomsten parameter-afhankelijk zijn, verbetert dynamische capaciteitsplanning aanzienlijk zowel de benutting van het systeem, als het deel van de patiënten dat een afspraak krijgt binnen de toegangstijdnorm.

\section{Deel V Optimaliseren van het gebruik van spreekkamers}

Spoedeisende hulp-afdelingen ( $\mathrm{SEHs}$ ) hebben regelmatig te maken met overbelasting, waardoor gezondheidsrisico's kunnen ontstaan. Op een SEH gebruiken artsen meerdere spreekkamers tegelijkertijd; wanneer een patiënt moet wachten op diagnostische tests, onderzoekt de arts andere patiënten. Dikwijls is er een disbalans in de toewijzing van spreekkamers aan artsen, wat gevolgen heeft voor de blokkeringskans, wachttijd en verblijftijd van patiënten.

In Hoofdstuk 10 gebruiken we een wachtrijmodel in willekeurige omgeving om de verwachte verblijftijd van patiënten te analyseren voor verschillende spreekkamertoewijzingspolitieken en werkroutines van de artsen. We valideren onze resultaten voor tijdsafhankelijke aankomsten, die kenmerkend zijn voor een SEH, met behulp van discrete event simulatie. Het blijkt dat een arts altijd twee of meer spreekkamers in parallel toegewezen moet krijgen. Bovendien kan een arts het best nieuwe patiënten onderzoeken, in plaats van patiënten die hij al eerder gezien heeft.

We breiden het onderzoek van Hoofdstuk 10 uit in Hoofdstuk 11 door een geheeltallig lineair programmeringsmodel met een rollende horizon op te lossen, zodat we tijdsafhankelijke aankomsten en patiënt- en arts-afhankelijke behandeltijden kunnen includeren in het model. Daarnaast onderzoeken we verschillende speciale instanties van het systeem, om inzicht te krijgen in welke werkroutine het beste resultaat oplevert voor welk type systeem. Uit de numerieke resultaten voor de realistische SEH instanties blijkt dat artsen het best de patiënten kunnen prioriteren die ze al eerder gezien hebben. Deze werkroutine is precies het tegenovergestelde van de routine die in Hoofdstuk 10 de beste resultaten opleverde. Hieruit concluderen we dat het onwaarschijnlijk is dat er één routine is die optimaal is voor alle SEH-systemen.

\section{Conclusie}

Het optimaliseren van de efficiëntie van logistieke processen in de zorg verbetert zowel de patiëntvriendelijkheid als de kwaliteit van zorg doordat de zorg toegankelijker wordt en afspraken beter op elkaar afgestemd worden. Daarnaast biedt het ziekenhuizen de kans om meer patiënten te behandelen met dezelfde capaciteit. Dit proefschrift bevat zowel theoretische als praktische toepassingen van 
OR methodieken die de logistieke besluitvorming in ziekenhuizen ondersteunen. Het onderzoek in dit proefschrift draagt bij aan het omvangrijke aantal artikelen over OR methodieken die toegepast zijn op de zorg. Daarnaast beschrijft dit proefschrift pragmatische, implementatie-gerichte onderzoeken en ervaringen met implementatie van onderzoeksresultaten in een ziekenhuis. De wiskundige methodieken in dit proefschrift zijn inventief en pragmatisch, en focussen op het menselijke aspect van de zorg door rekening te houden met de voorkeuren van zowel patiënten als artsen. Dit proefschrift is geschreven met de ambitie een brug te slaan tussen theorie en praktijk, zodat academici en zorgprofessionals samen kunnen werken aan de voortdurende verbetering van de efficiëntie van zorglogistiek. 



\section{About the author}

Maartje van de Vrugt was born in Amersfoort, the Netherlands, on December 18, 1988. In 2000 she began her preparatory university education (in Dutch: Atheneum) at Johannes Fontanus College in Barneveld, during which she, together with two classmates, won the regional preliminaries of the European Union Science Olympiad and Aarde.nu secondary school thesis competition. She obtained her diploma in 2006, after which she started studying Applied Mathematics at the University of Twente. She obtained her bachelor's degree with a thesis on optimal saving strategies for a personal pension fund in June 2009, and started her master's with specialization in Operations Research. From 2007 to 2009, Maartje followed the University of Twente Honors Program, for which she co-authored the (Dutch) book 'Hier en nu in ver verschiet'. Through the Honors Program, Maartje met Prof. dr. Richard J. Boucherie and got interested in doing Ph.D. research. Her internship at the consultancy firm 'Witteveen+Bos', on model based aeration policies for wastewater treatment plants, was awarded the KIVI NIRIA/UT price. She defended her master's thesis on blocking probabilities in Erlang loss queues with advance reservation (which is the basis for Chapter 5) in August 2011.

Maartje started her Ph.D. research in September 2011 at the Center for Healthcare Operations Improvement and Research (CHOIR) of the University of Twente. In April 2013 she started at the Jeroen Bosch Hospital, where she was involved in several consultancy projects besides her Ph.D. research. Maartje was awarded a scholarship from the 'Stichting Jo Kolk Studiefonds', which enabled her to visit Dr. Ilze Ziedins at the University of Auckland, New Zealand from January until March 2015. In the beginning of 2016, she visited Dr. Ilze Ziedins again, completing the basis for Chapter 11. Maartje's Ph.D. research culminates with this thesis.

For the next step in her career Maartje aims for a hybrid job, focusing on pragmatic research and it's implementation at hospitals, but with some time available for academic research. 



\section{List of publications}

L. Berrevoets, N.M. van de Vrugt, and G. de Vries. Bezettingsgraad. [In Dutch] In B. Berden, L. Berrevoets, W. Winasti, editors, Capaciteitsplanning in de zorg, chapter 13, Bohn Stafleu van Loghum, Houten, the Netherlands, 2016.

A. Braaksma, N.M. van de Vrugt, and R.J. Boucherie. Online appointment scheduling: a taxonomy and review. Submitted.

Basis for Chapter 4 .

S.M. Cornelissen, N.M. van de Vrugt, E.A.A. Smits, and T.P.J. Timmerhuis. Slim samenwerken aan een evenwichtige bedbezetting. [In Dutch] Submitted.

Basis for Chapter 3.

C.M. Laan, N.M. van de Vrugt, R.J. Boucherie, and J. Olsman. Static and dynamic appointment scheduling to meet access time norms. Submitted.

Basis for Chapter 9.

N.M. van de Vrugt and R.J. Boucherie. Assigning treatment rooms at the Emergency Department. Operations Research for Health Care, 8:62-70, 2016.

Basis for Chapter 10.

N.M. van de Vrugt, R.J. Boucherie, T.J. Smilde, M. de Jong, and M. Bessems. Rapid diagnoses at the Breast center of Jeroen Bosch Hospital: a case study invoking queueing theory and discrete event simulation. Submitted.

Basis for Chapter 6 .

N.M. van de Vrugt, N. Litvak, and R.J. Boucherie. Blocking probabilities in Erlang loss queues with advance reservation. Stochastic Models, 30(2):187-196, 2014.

Basis for Chapter 5.

N.M. van de Vrugt, S.T. Luen-English, W.A.P. Bastiaansen, S. Kleinluchtenbeld, W.T.P. Lardinois, M.H. Pots, D.J. Schoonbergen, E.W. Hans, J.L. Hurink, R.J. Boucherie. Simultaneously scheduling tasks and gynecologists to improve patient appointment scheduling. Submitted.

Basis for Chapter 8. 
N.M. van de Vrugt, P. Matel, R.J. Boucherie, P. van Engelen, T. Beukman, and J. de Laat. Verbeterde afsprakenplanning voor patiënt en gipsverbandmeester. [In Dutch] In dit Verband, 26(1): 6-7, 2016.

Basis for Chapter $\%$.

N.M. van de Vrugt, A.J. Schneider, M.E. Zonderland, D.A. Stanford, and R.J. Boucherie. Operations Research for Hospital Wards and its Integration into Practice. Submitted.

Basis for Chapter 2.

N.M. van de Vrugt and I. Ziedins. Sequentially assigning and prioritizing patients at Emergency Departments. Working paper.

Basis for Chapter 11. 